\title{
SENSIBILIDADE EM FLUXO DE POTÊNCIA ÓTIMO
}

\author{
Edmarcio Antonio Belati
}

\author{
Tese apresentada à Escola de Engenharia \\ de São Carlos, da Universidade de São \\ Paulo, como parte dos requisitos para \\ obtenção do título de Doutor em \\ Engenharia Elétrica.
}

Orientador: Prof. Dr. Geraldo R. M. da Costa 
“O que vale na vida não é o ponto de partida e sim a caminhada. Caminhando e semeando, no fim terás o que colher."

Cora Coralina 
Agradeço a Deus por me dar saúde e forças para finalizar mais uma etapa da minha vida $e$ coragem para continuar. 
Dedico aos meus pais e à minha irmã pelo apoio e carinho durante esta etapa da minha vida. 


\section{AGRADECIMENTOS}

Ao Professor Dr. Geraldo Roberto Martins da Costa, por sua valiosa orientação, paciência, disponibilidade e amizade durante o desenvolvimento deste trabalho.

À Professora Dr. a Edméa Cássia Baptista, pelo "apoio matemático”, pela amizade e companheirismo.

Aos amigos do LOSEP: Alessandra, Vanusa, Thales, Cristiano, Sandra, Sereno, Claudinho; e aos do Departamento de Engenharia Elétrica, pela valiosa convivência.

A todos os professores e funcionários do Departamento de Engenharia Elétrica da EESC-USP-São Carlos, pela colaboração e atenção.

A todos os amigos e amigas de república, da cidade e da EESC que conviveram comigo durante esse período, torcendo, apoiando e divertindo, meus agradecimentos.

À FAPESP pelo apoio financeiro, indispensável à realização deste trabalho. 


\section{ÍNDICE}

1 INTRODUÇÃO.....................................................................................

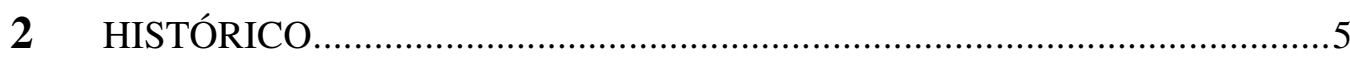

2.1 HISTÓRICO DO PROBLEMA DE FLUXO DE POTÊNCIA

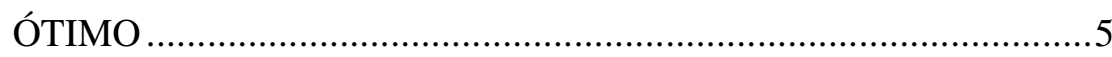

2.2 HISTÓRICO DE SENSIBILIDADE EM SISTEMAS ELÉTRICOS

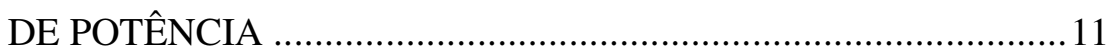

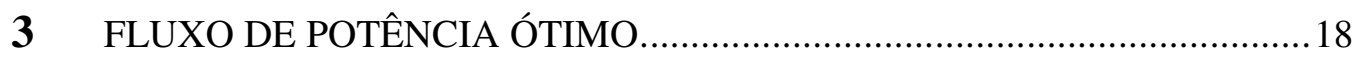

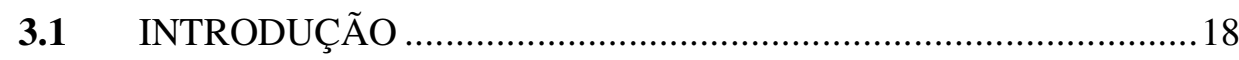

3.2 O PROBLEMA DE FLUXO DE POTÊNCIA ÓTIMO .....................18

3.3 METODOLOGIA DE RESOLUÇÃO APLICADA AO PROBLEMA

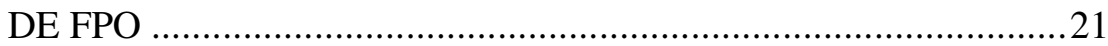

3.4 A FUNÇÃO LAGRANGIANA AUMENTADA BARREIRA

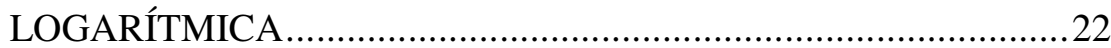

3.5 O MÉTODO DA FUNÇÃO LAGRANGIANA AUMENTADABARREIRA LOGARÍTMICA ………………………………........25

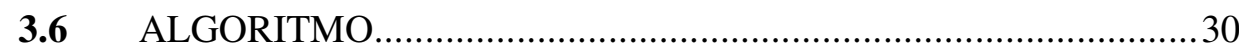

3.7 TÉCNICAS DE ESPARSIDADE.......................................................

3.8 IMPLEMENTAÇÃO COMPUTACIONAL …………………..........32

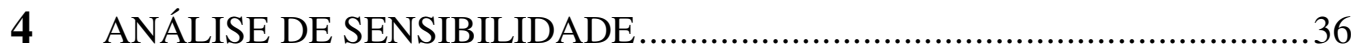

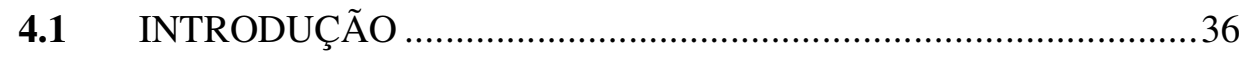

4.2 FORMULAÇÃO MATEMÁTICA DA SENSIBILIDADE

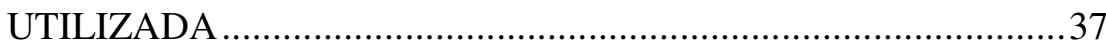

4.3 APLICAÇÃO DA METODOLOGIA SFPO......................................40

4.3.1 OBTENÇÃO DO PONTO ÓTIMO ........................................41

4.3.2 ESTIMAÇÃO DO NOVO PONTO VIA SFPO ………….....43

4.3.3 VALIDAÇÃO DA METODOLOGIA SFPO .........................44 
5 SENSIBILIDADE APLICADA AO PROBLEMA DE FPO . .47

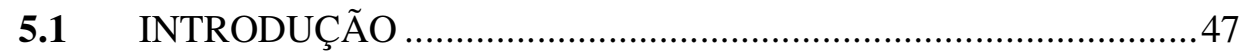

5.2 APLICAÇÃO DA METODOLOGIA SFPO AO PROBLEMA DE FLUXO DE POTÊNCIA ÓTIMO

5.3 RESOLUÇÃO DO PROBLEMA DE FLUXO DE POTÊNCIA ÓTIMO PERTURBADO VIA SFPO 51

5.4 TÉCNICA DE ESPARSIDADE UTILIZADA ………………...........51

5.5 IMPLEMENTAÇÃO COMPUTACIONAL ……………………......53

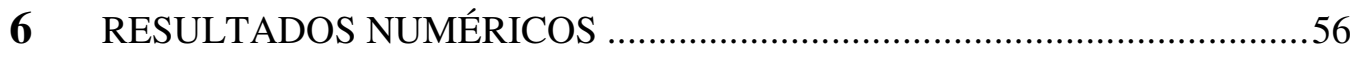

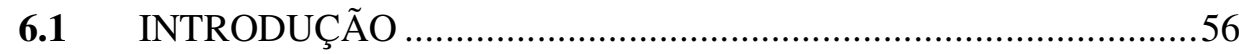

6.2 EXEMPLO 1: SISTEMA 3 BARRAS .............................................57

6.3 EXEMPLO 2: SISTEMA IEEE 14 BARRAS...................................67

6.4 EXEMPLO 3: SISTEMA IEEE 30 BARRAS.................................... 82

6.5 EXEMPLO 4: SISTEMA CESP 53 BARRAS .................................. 85

6.6 EXEMPLO 5: SISTEMA IEEE 118 BARRAS................................... 88

6.7 EXEMPLO 6: SISTEMA IEEE 162 BARRAS.................................90

6.8 EXEMPLO 6: SISTEMA IEEE 300 BARRAS.................................90

6.9 EXEMPLO 7: SISTEMA 9 BARRAS ...........................................92

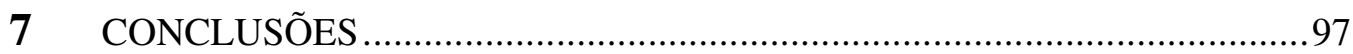

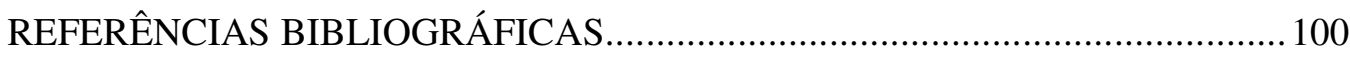

APÊNDICE A - BANCO DE DADOS E TABELAS DE CONVERGÊNCIA DOS SISTEMAS ELÉTRICOS UTILIZADOS

APÊNDICE B - RESULTADOS OBTIDOS 


\section{LISTA DE FIGURAS}

Figura 3.1 - Módulos do programa do Método da Função Lagrangiana AumentadaBarreira Logarítmica, aplicado ao problema de FPO..........................33

Figura 4.1 - Solução ótima do problema, $\varepsilon_{1}=0$...........................................42

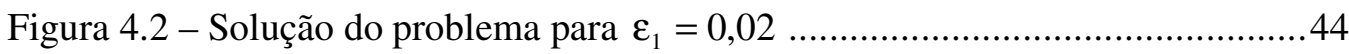

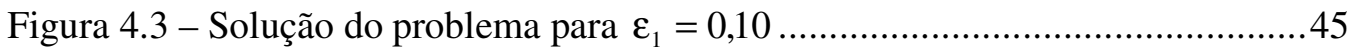

Figura 4.4 - Solução do problema com $\varepsilon_{1}=0,20$.............................................46

Figura 5.1 - Módulos do programa da metodologia SFPO …...............................54

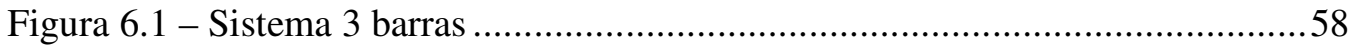

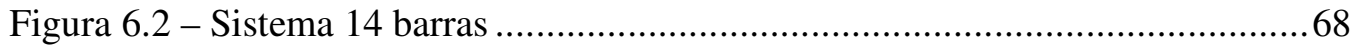

Figura 6.3 - Perdas de potência ativa na transmissão...........................................69

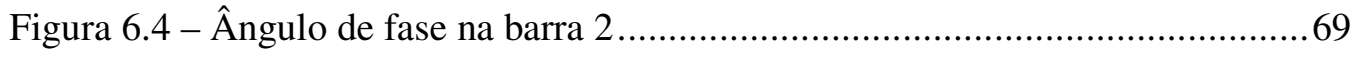

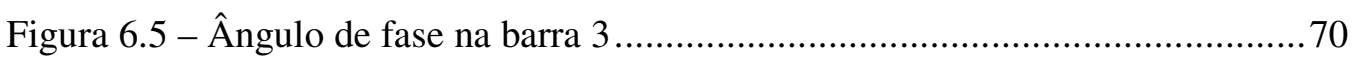

Figura 6.6 - Ângulo de fase na barra 4 ........................................................... 70

Figura 6.7 - Ângulo de fase na barra 5 ............................................................ 70

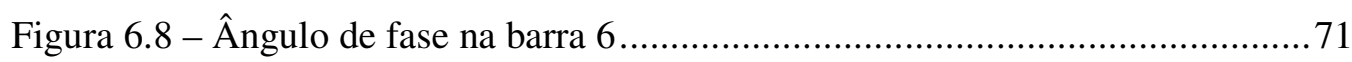

Figura 6.9 - Ângulo de fase na barra 7 ......................................................... 71

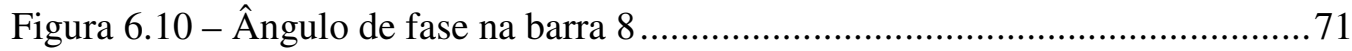

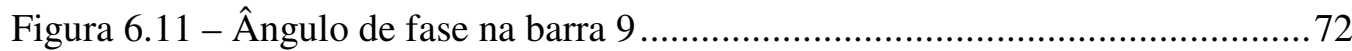

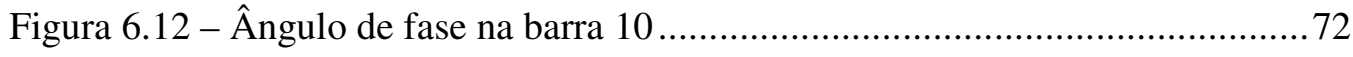

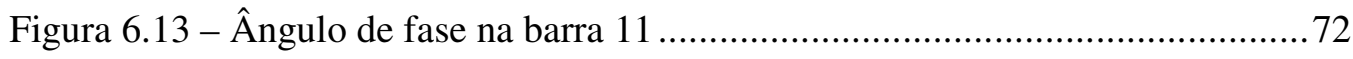

Figura 6.14 - Ângulo de fase na barra 12 ....................................................... 73

Figura 6.15 - Ângulo de fase na barra 13 ......................................................... 73

Figura 6.16 - Ângulo de fase na barra 14 ........................................................... 73

Figura 6.17 - Magnitude da tensão na barra 1 ................................................... 74

Figura 6.18 - Magnitude da tensão na barra 2 ............................................... 74

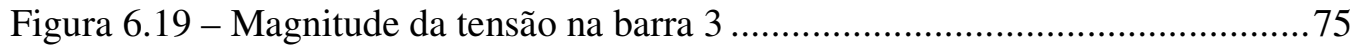

Figura 6.20 - Magnitude da tensão na barra 4 .................................................. 75

Figura 6.21 - Magnitude da tensão na barra 5 .................................................... 75 


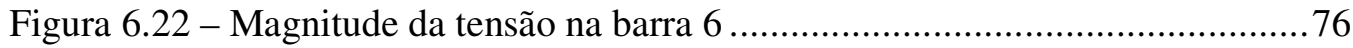

Figura 6.23 - Magnitude da tensão na barra 7 .................................................. 76

Figura 6.24 - Magnitude da tensão na barra 8 .................................................. 76

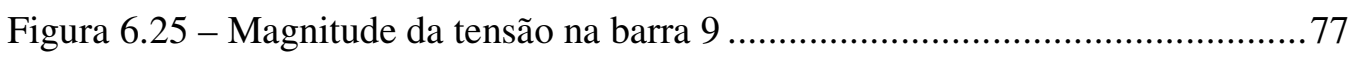

Figura 6.26 - Magnitude da tensão na barra 10 ................................................. 77

Figura 6.27 - Magnitude da tensão na barra 11 .................................................... 77

Figura 6.28 - Magnitude da tensão na barra 12 .................................................78

Figura 6.29 - Magnitude da tensão na barra 13 ................................................... 78

Figura 6.30 - Magnitude da tensão na barra 14 ................................................. 78

Figura 6.31 - Geração de potência reativa na barra 2 ...........................................79

Figura 6.32 - Geração de potência reativa na barra 3 ............................................... 80

Figura 6.33 - Geração de potência reativa na barra 6 .......................................... 80

Figura 6.34 - Geração de potência reativa na barra 8 ............................................ 80

Figura 6.35 - Maior erro da potência ativa e reativa .......................................... 81

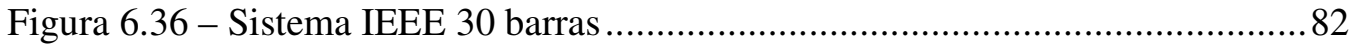

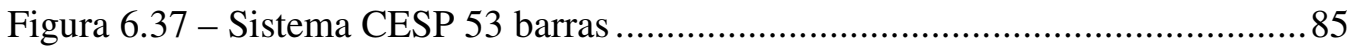

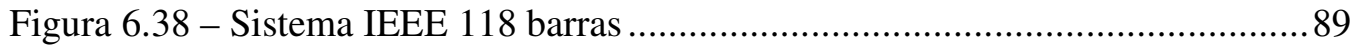

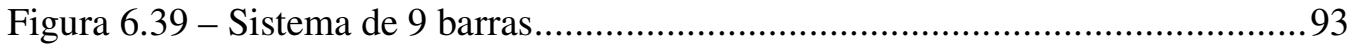




\section{LISTA DE TABELAS}

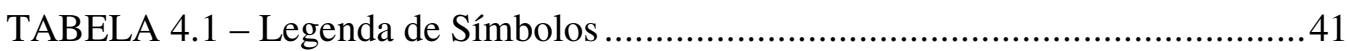

TABELA 4.2 - Solução ótima do problema para $\varepsilon_{1}=0$.....................................42

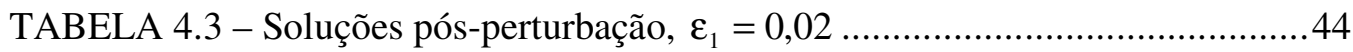

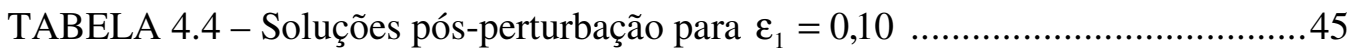

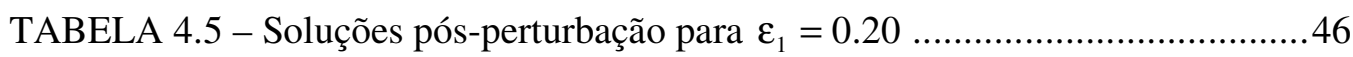

TABELA 6.1 - Condição inicial do sistema 3 barras .............................................58

TABELA 6.2 - Limite das variáveis do sistema 3 barras.......................................58

TABELA 6.3 - Variáveis primais, geração de potência reativa e perdas na transmissão obtidas via FPO para $(\varepsilon=0,0)$...................................58

TABELA 6.4 - Variáveis duais obtidas via FPO ...............................................59

TABELA 6.5 - Alterações nos parâmetros da barras 3 .........................................65

TABELA 6.6 - Variáveis primais e geração de potência reativa após a perturbação 67

TABELA 6.7 - Variáveis duais após a perturbação...................................................67

TABELA 6.8 - Solução ótima do sistema 30 barras $(\varepsilon=0,0)$ usada pela SFPO ........83

TABELA 6.9 - Solução do sistema 30 barras estimada pela SFPO após a

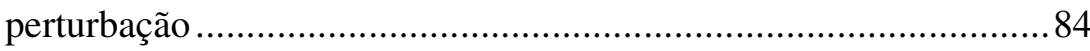

TABELA 6.10 - Solução ótima do sistema CESP $(\varepsilon=0,0)$ usada pela SFPO ...........86

TABELA 6.10 - Solução do sistema CESP estimada pela SFPO após a perturbação87

TABELA 6.11 - Análise de sensibilidade da injeção de potência reativa no sistema em relação ao aumento da carga ..............................................91

TABELA 6.12 - Solução ótima do sistema de 9 barras $(\varepsilon=0,0)$ usada pela SFPO....94

TABELA 6.13 - Solução do sistema de 9 barras estimada pela SFPO após a perturbação....................................................................... 94

TABELA 6.14 - Comparação das variáveis primais, obtidas via DOPA e via SFPO para o problema perturbado .95

TABELA 6.15 - Comparação dos fluxos de potência ativa e reativa nas linhas, obtidos via DOPA e via SFPO para o problema perturbado .........95 


\section{LISTA DE ABREVIATURA E SIGLAS}

SEP - Sistema Elétrico de Potência;

FPO - Fluxo de Potência Ótimo;

KKT - Karush-Kuhn-Tucker;

DE - Despacho Econômico;

UPFC - Unified Power Flow Controller;

FACTs - Flexible AC Transmission Systems;

SFPO - Sensibilidade Aplicada ao Problema de Fluxo de Potencio Ótimo;

PIQM - Pontos Interiores Quadráticos Melhorado;

MW - Mega Watts;

MVAr - Mega Volt Ampere Reativo;

AC - Corrente Alternada;

NBCCR - Número de Barras de Carga e Controle de Reativo;

NBC - Número de Barra de Carga;

NBCR - Número de Barras de Controle de Reativo;

NB - Número de Barras;

NT - Número de Transformadores;

PNL - Problema de Programação Não Linear;

PV - Barra de Controle de Reativo;

PQ - Barra de Carga;

CAG - Controlador Automático de Geração;

IEEE - International Electrical and Electronics Engineers;

CESP - Companhia Energética de São Paulo;

p.u. - por unidade;

PECO - Philadelphia Electric Company. 


\section{LISTA DE SÍMBOLOS}

$\Omega \mathrm{k}$ - Conjunto de barras vizinhas à barra K.

$\mathrm{V}_{\mathrm{k}}$ - Tensão da barra k;

$\theta_{\mathrm{k}}-$ Ângulo de tensão nodal da barra k;

$\mathrm{t}_{\mathrm{k}}-$ Tap $\mathrm{k}$ do transformador da $\mathrm{k}$;

$\mathrm{P}_{\mathrm{k}}$ - Potência ativa liquida (geração menos carga) na barra k;

$\mathrm{Q}_{\mathrm{k}}$ - Potência reativa líquida na barra $\mathrm{k}$;

$\mathrm{Q}_{\mathrm{k}}^{\text {sh }}$ - Componente de injeção de potência reativa devido ao elemento shunt da barra $\mathrm{k}$;

$\mathrm{P}_{\mathrm{km}}$ - Fluxo de potência ativa no ramo k - m;

$\mathrm{Q}_{\mathrm{km}}$ - Fluxo de potência reativa no ramo k - m;

$\mathrm{g}_{\mathrm{km}}$ - Condutância série do ramo k - m;

$\mathrm{x}$ - Vetor das variáveis primais do problema;

$\mathrm{g}(\mathrm{x})$ - Vetor das equações do fluxo de potência;

$\mathrm{h}(\mathrm{x})$ - Vetor das equações funcionais do fluxo de potência;

$f(x)$ - Função objetivo;

$\mathrm{z}$ - Variáveis de folga;

su - Variáveis de folga;

si - Variáveis de excesso;

$\delta$ - Fator de barreira;

c - Fator de barreira;

$\lambda$ - Vetor dos multiplicadores de Lagrange relacionados com as restrições de igualdade;

$\mu$ - Vetor dos multiplicadores de Lagrange relacionados com as restrições de desigualdade;

$\pi \mathrm{u}$ - Vetor dos multiplicadores de Lagrange relacionados com as restrições de desigualdade canalizadas (barreira); 
$\pi \mathrm{i}$ - Vetor dos multiplicadores de Lagrange relacionados com as restrições de desigualdade canalizadas (barreira);

$\mathrm{R}$ - Conjunto dos números reais;

$\in-$ Pertence a;

$\nabla_{\mathrm{x}}-$ Vetor gradiente com relação à $\mathrm{x}$;

i, j, k, 1, m - Número Inteiro;

$\alpha_{\mathrm{p}}$ - Passo primal;

$\alpha_{d}-$ Passo dual;

S - Matriz Sensibilidade;

E - Vetor perturbação;

$\mathrm{R}_{\mathrm{S}}-$ Vetor resposta;

$\varepsilon_{\mathrm{k}}^{\mathrm{P}}-$ Vetor perturbação relacionado com as equações de igualdade das potências ativas;

$\varepsilon_{\mathrm{k}}^{\mathrm{Q}}-$ Vetor perturbação relacionado com as equações de igualdade das potências reativas;

$\varepsilon_{\mathrm{i}}-$ Vetor que relaciona os $\varepsilon_{\mathrm{k}}^{\mathrm{P}}$ e os $\varepsilon_{\mathrm{k}}^{\mathrm{Q}}$;

* - Indicação de variável ótima. 


\section{RESUMO}

BELATI, E. A. (2003). Sensibilidade em Fluxo de Potência Ótimo. São Carlos, 2003. 106p. Tese (Doutorado) - Escola de Engenharia de São Carlos, Universidade de São Paulo.

Neste trabalho propomos uma abordagem para a resolução do problema de Fluxo de Potência Ótimo (FPO) perturbado. A metodologia consiste na obtenção da solução ótima para o problema inicial via um programa de FPO, e na utilização de sensibilidade para estimar novas soluções depois de ocorridas algumas perturbações no problema. Essas perturbações são variações de carga em uma ou mais barras do sistema. A técnica de sensibilidade está baseada nas informações de segunda ordem e nas condições de otimalidade. A obtenção da solução após ocorrerem perturbações no sistema é direta e não necessita de parâmetros iniciais e de correção, como penalidade e barreira, utilizados nos programas de FPO convencionais. Os resultados numéricos apresentados evidenciam o potencial desta metodologia para resolução do problema de FPO perturbado.

Palavras - chave: Análise de Sensibilidade; Fluxo de Potência Ótimo; Perturbação; Perdas de Potência Ativa na Transmissão; Função Lagrangiana Aumentada; Pontos Interiores. 


\section{ABSTRACT}

BELATI, E. A. (2003). Sensitivity in Optimal Power Flower. São Carlos, 2003. 106p. Tese (Doutorado) - Escola de Engenharia de São Carlos, Universidade de São Paulo.

An approach to solve the perturbated Optimal Power Flow (OPF) problem is proposed in this study. The methodology consists in obtaining the optimal solution for the initial problem via a program of OPF, and using sensitivity to estimate new solutions after the occurrence of some perturbations in the problem. These perturbations consist in load variations in some buses of the system. The sensitivity technique is based on both the information of second order and otimality conditions. The computation of the solutions after the occurrence of perturbations in the system does not depend of initial and correction parameters such as penalty and barrier used in the conventional OPF programs. The numerical results demonstrate the potential of this methodology for the solution of the perturbated OPF problem.

Keywords: Sensitivity Analysis; Optimal Power Flow Problem; Perturbation; Losses of Active Power in the Transmission; Augmented Lagrangian Function; Interior Points. 


\section{Capítulo 1}

\section{INTRODUÇÃO}

O estudo das metodologias para se administrar uma perturbação local - análise de sensibilidade referente a mudanças do problema original - é uma exigência de várias disciplinas científicas. Em muitos casos, o estado de uma solução não pode ser inteiramente compreendido sem a análise de sensibilidade. Isso tem sido bem reconhecido desde o princípio das investigações cientificas e vem sendo explicitamente utilizado por matemáticos. Na matemática, técnicas de sensibilidade são usadas para se obter condições de otimalidade, resultados duais, soluções de algoritmos, convergência e taxa de convergência, em adição a suas mais óbvias e imediatas aplicações em estimar soluções com diferentes parâmetros.

Em sistemas estáticos de energia elétrica, há dois tipos de sensibilidades predominantes: a sensibilidade de uma variável elétrica em relação à outra, como por exemplo, a tensão, $V_{i}$, na barra $i$, em relação à injeção de reativo, $Q_{j}$, na barra $j$, e a sensibilidade da função objetivo, $\mathrm{f}(\mathrm{x})$, em relação às variáveis elétricas, como por exemplo, as perdas ativas na transmissão, em relação à injeção de potencia $P_{i}$ na barra i. O conhecimento dessas sensibilidades é de grande importância para várias 
áreas do Sistema Elétrico de Potência (SEP). A sensibilidade pode ser aplicada no planejamento do sistema, na tarifação, no ajuste de uma solução factível do fluxo de carga para otimizar a solução, na implementação de uma técnica de despacho em tempo real aplicável a casos em que há pequenas variações na demanda, entre outras aplicações.

Neste trabalho utilizaremos a análise de sensibilidade para encontrar um novo ponto de operação para o problema de Fluxo de Potência Ótimo (FPO) depois de ocorridas pequenas perturbações no sistema. Este estudo de sensibilidade também permitirá a análise do sistema de maneira fácil e direta, isto é, a verificação do novo desempenho da rede (função objetivo) em relação às pequenas variações da demanda.

A metodologia utilizada consiste, primeiramente, na obtenção de uma solução ótima inicial para o problema via um programa FPO, e na utilização de sensibilidade para estimar novas soluções depois de ocorridas algumas perturbações na demanda do sistema. Essas perturbações poderão ser um acréscimo ou corte de carga em uma ou mais barras do sistema. A utilização da sensibilidade permitirá encontrar novos pontos de operação sem a necessidade de se reprocessar o FPO. Os maiores benefícios da técnica, em comparação aos FPOs, são a rapidez e robustez das soluções, a não necessidade da utilização de parâmetros de entrada e de correção como penalidade e barreira. Essas características fazem com que a aplicação da metodologia possa ser viável para as empresas de energia elétrica, pois para se 
executar o programa não há a necessidade de operadores com conhecimentos de otimização.

O programa de FPO utilizado para a obtenção da solução inicial, consiste em uma abordagem da Função Lagrangiana Aumentada denominada de Método da Função Lagrangiana Aumentada-Barreira Logarítmica. Nessa abordagem, as restrições canalizadas são tratadas por uma função barreira; as restrições de igualdade, pelo método de Newton; e as desigualdades, pela função Lagrangiana Aumentada. O método preserva as características da função Lagrangiana Aumentada, pois caminha pelo exterior da região viável, obedecendo às restrições de canalização. Devido ao processo de linearização, é gerado um sistema matricial do tipo $\mathrm{Ax}=\mathrm{b}$. A matriz, A, é esparsa e simétrica de valor e posição. Essa característica é explorada através da técnica de esparsidade MA57 $^{1}$, que visa melhorar o desempenho computacional. A convergência do algoritmo está diretamente ligada aos fatores iniciais de barreira e penalidade e de seus parâmetros de correção, o que não acontece na técnica de sensibilidade empregada.

A técnica de sensibilidade é baseada nas informações de segunda ordem e nas condições de otimalidade. A solução do problema é obtida após a resolução de um sistema matricial, do tipo $\mathrm{Ax}=\mathrm{b}$. A matriz, A, é esparsa e simétrica de posição, porém não de valores. Para resolver o sistema, empregamos a técnica de esparsidade MA28 ${ }^{1}$, que explora esta característica da matriz.

\footnotetext{
1 Resolve um sistema simétrico e esparso de equações lineares. Catálogo disponível em http://www.hsl-library.com/cat_hs12002.pdf.
} 
Este trabalho é dividido em 7 capítulos. Neste capítulo, apresentamos a motivação, o objetivo e a organização do trabalho. No capítulo 2 , faremos uma revisão bibliográfica sobre FPO e sensibilidade. No capítulo 3, mostraremos a metodologia utilizada na resolução do problema de FPO. No capítulo 4, desenvolveremos a técnica de sensibilidade utilizada neste trabalho. No capítulo 5, aplicaremos a técnica de sensibilidade, ao problema de FPO. No capítulo 6, apresentamos os resultados numéricos do método aos sistemas elétricos de 3 barras, 9 barras, IEEE 14 barras, IEEE 30 barras, CESP 53 barras, IEEE 118 barras, IEEE 162 barras e para o sistema IEEE 300 barras. Finalmente, no capítulo 7, apresentamos as conclusões do nosso trabalho e as perspectivas de continuidade deste. 


\section{HISTÓRICO}

Apresentaremos a seguir um levantamento bibliográfico do problema de fluxo de potência ótimo e das técnicas de sensibilidade aplicadas em sistemas elétricos de potência.

\subsection{HISTÓRICO DO PROBLEMA DE FLUXO DE POTÊNCIA ÓTIMO}

O problema de FPO foi proposto por CARPENTIER (1962), no início da década de 60, a partir do problema de despacho econômico (DE). Historicamente, o problema de despacho econômico, foi o precursor do problema de fluxo de carga ótimo, o qual marcou o fim do período clássico do despacho econômico. Assim, o problema de DE passou a ser abordado como um caso particular do FPO. Desde então, muitos trabalhos foram realizados na tentativa de resolvê-lo. Mas, até o presente, não existe uma abordagem realmente robusta, confiável e rápida, que atenda às necessidades dos centros de operação das concessionárias de energia elétrica. 
Uma das primeiras abordagens propostas para resolver o problema de fluxo de potência ótimo foi feita por DOMMEL \& TINNEY (1968). Eles propuseram o método do gradiente reduzido para resolver o problema de fluxo de potência ótimo. Tal método consiste numa direção de passo descendente aplicado ao subespaço tangente das equações não lineares do fluxo de potência. É um método em que, as equações do fluxo de potência são resolvidas pelo método de Newton após mudanças nas variáveis de controle. Nesse método as restrições de igualdade são associadas à função Lagrangiana, através dos multiplicadores de Lagrange, enquanto que as restrições de desigualdade são tratadas através de parâmetros de penalidade. O método tem uma eficiência de primeira ordem para a minimização da função objetivo. Apesar de seu rigor matemático, esta abordagem apresenta convergência lenta, “zig-zagueando" próximo da solução ótima. Para a atualização dos novos valores das variáveis de controle utiliza-se um passo, que é determinado por uma busca unidimensional. O método apresenta dificuldades na modelagem dos componentes, tal como taps dos transformadores que são considerados no fluxo de carga e não na rotina de otimização. O método foi capaz de resolver problemas com até 500 barras. Este trabalho foi pioneiro e serviu como referência à produção de muitas abordagens para resolução do FPO.

SASSON. (1969) apresentou um combinado do método de Powell e FletcherPowell. O método de Powell é usado para resolver problemas de otimização restrita, enquanto que o método de Fletcher-Powell é usado para resolver problemas de otimização irrestrita. Ambos propuseram uma técnica de segunda ordem para resolver o problema, ou seja, utilizar a matriz Hessiana. Esta técnica tem o objetivo 
de tornar o problema restrito em irrestrito, penalizando todas as restrições de desigualdade que são violadas e as de igualdade que ainda estão longe de zero. A ação da penalidade faz com que o valor da função objetivo tenda a aumentar quando uma restrição é violada. O algoritmo tentará minimizar a violação e, trazer as variáveis de volta para a região factível. Durante o processo de convergência, estes fatores de penalidade vão aumentando, o que pode causar mal condicionamento da matriz Hessiana, ou seja, elementos muito elevados na diagonal principal, comprometendo a convergência do método. O método foi testado no sistema IEEE 30-barras.

O método de penalidade, apresentado por SASSON et al. (1973), surgiu com o objetivo principal de acelerar a convergência do método utilizado por DOMMEL \& TYNNEY (1968). A idéia desse método consiste em transformar o problema restrito em irrestrito, minimizando, assim, uma função auxiliar formada pela função objetivo e pela função de penalidade, que consiste nas restrições de igualdade e desigualdade penalizadas. A cada iteração são aumentados os fatores de penalidade, a matriz Hessiana da função auxiliar é calculada, e as variáveis são atualizadas. O processo é repetido até que todas as restrições estejam satisfeitas. A matriz Hessiana recebe influência direta dos fatores de penalidade; logo, para valores elevados dos fatores de penalidade, ela pode se tornar mal condicionada.

RASHED \& KELLY. (1974), propuseram um método em que foram empregadas técnicas de programação não-linear para minimizar a função objetivo, isto é, as perdas na transmissão e o custo de geração. Os multiplicadores de Lagrange 
e o método de Newton são utilizados na solução do problema de fluxo de potência ótimo. Nesse método, as restrições de igualdade, representadas pelas equações de fluxo de potência, são incorporadas à função Lagrangiana através dos multiplicadores de Lagrange, enquanto que as restrições de desigualdade são incorporadas através de parâmetros de penalidades. Para a correção das variáveis, utiliza-se a matriz Hessiana e o vetor gradiente da função Lagrangiana. Este trabalho foi uma continuação do trabalho de DOMMEL \& TINNEY (1968). O método foi testado em um sistema de 179 barras.

SUN et al. (1984), utilizaram uma formulação explicita do método de Newton para resolução do problema de FPO. A cada iteração, a função Lagrangiana é aproximada por uma quadrática. O conjunto de restrições de desigualdade é dividido em dois grupos: restrições penalizadas, introduzidas na função objetivo através de termos de penalidade quadráticos, e restrições ativas na solução, incorporadas ao conjunto de restrições de igualdade do problema. A solução do problema ocorre quando as condições de otimalidade de KKT estão satisfeitas, e as equações do fluxo de carga convencional estão dentro das tolerâncias determinadas. O método apresenta convergência de segunda ordem e tem como deficiência a necessidade de identificar as restrições de desigualdade que são ativas na solução. O método foi testado num sistema de 912 barras.

SANTOS et al. (1988) desenvolveram um trabalho em que o problema do fluxo de potência ótimo é resolvido pelo método da Lagrangiana Aumentada, o qual está baseado nas técnicas de programação não-linear. A trajetória de convergência do 
método é pela região infactível do problema. A cada iteração a função Lagrangiana Aumentada é minimizada em relação às variáveis primais, utilizando o método de Newton. Os multiplicadores de Lagrange são atualizados visando a maximização da função Lagrangiana Aumentada Dual, associada ao problema original. Este método não necessita de parâmetros de penalidade muito elevados. A solução é obtida quando todas as restrições de igualdade e de desigualdade estão sendo satisfeitas, dentro de uma tolerância especificada.

MONTICELLI \& LIU (1992) propuseram uma nova abordagem do método de Newton, combinando o método dos multiplicadores de Lagrange e o método da função penalidade. A proposta é basicamente a mesma de SUN et al. (1984), a diferença está na utilização de uma penalidade adaptativa que assegura que a matriz Hessiana seja definida positiva ao longo do processo de solução, sem afetar a convergência do problema.

GRANVILLE (1994) propôs o método Primal-Dual Barreira-Logarítmica, um método de pontos interiores, que consiste em encontrar o ponto ótimo satisfazendo às restrições canalizadas durante o processo iterativo. Utiliza multiplicadores de Lagrange para as restrições de igualdade e transforma as desigualdades em equações de igualdade, através do uso de variáveis de folga. Estas variáveis são incorporadas à função objetivo através da função barreira logarítmica e dos parâmetros de barreira, que tende a zero com o aumento do número de iterações. Este algoritmo apresenta muita sensibilidade quanto à escolha do parâmetro de barreira, podendo divergir em alguns casos. A solução é encontrada quando todas as restrições do problema original 
estão satisfeitas. Destaca-se também nesta mesma área de pontos interiores, o trabalho de WU et al. (1994), que sugere uma extensão do método primal-dual. Seu algoritmo é chamado de método preditor-corretor, e sua diferença em relação ao método primal-dual, está na introdução de termos não-lineares nas condições de otimalidade. O trabalho de QUINTANA et al. (1995), também contribuiu para o avanço das técnicas de otimização que utilizam pontos interiores.

COSTA (1997), propôs o método de Newton associado à Função Lagrangiana Aumentada, que basicamente associa os métodos propostos por SUN et al. (1984) e SANTOS et al. (1988). Este método não apresenta a necessidade de se identificar as restrições de desigualdade que estão ativas na solução, eliminando, assim, a deficiência do algoritmo de SUN et al. (1984). As restrições de igualdade são tratadas pelo método de Newton, e não são penalizadas. O método foi aplicado em sistemas de 57 e 662 barras.

TORRES \& QUINTANA (1998) propuseram a resolução do problema FPO pelo Método dos Pontos Interiores, usando coordenadas retangulares de tensão. Algumas variantes do problema de FPO, quando formuladas na forma retangular têm função objetivo e restrições quadráticas. Tais características permitem facilidades para iniciar e incorporar informações de alta ordem em um procedimento preditorcorretor que melhora o desempenho do método dos pontos interiores. A técnica proposta teve um bom desempenho computacional. 
MOMOH \& ZHU (1999) apresentaram em 1999 o método dos Pontos Interiores Quadráticos Melhorado (PIQM) aplicado à solução do problema de FPO. A abordagem pode trabalhar com funções objetivo que podem ser o despacho ótimo, planejamento de reativos e minimização das perdas. É realizado um procedimento de eliminação para se obter uma redução no problema de FPO, onde a função objetivo é uma função quadrática sujeita às restrições lineares. As características do método PIQM são a possibilidade de um ponto de partida geral ou seja, a não necessidade de um ponto inicial bom, como é requerido nos demais métodos de pontos interiores - e a rápida convergência. $\mathrm{O}$ método foi testado nos sistemas IEEE 14, 30 e 118 barras para o despacho ótimo, planejamento de reativos e minimização das perdas.

COSTA et al (2000) propuseram uma nova abordagem que melhora o método de Newton para o problema de FPO. No qual os multiplicadores de Lagrange são calculados diretamente do sistema linearizado. A fatoração é feita por elementos, ao invés de pela estrutura de blocos. As principais vantagens são o curto tempo de processamento computacional e a redução no uso de memória. Foram feitos testes numéricos para sistemas IEEE de 14, 30, 118 barras e para o sistema brasileiro de 810 barras, que mostraram a eficiência do método proposto.

\subsection{HISTÓRICO DE SENSIBILIDADE EM SISTEMAS ELÉTRICOS DE POTÊNCIA}

É de fundamental importância, em um sistema de controle, o completo entendimento de como as variações no sistema afetam seu estado. Tal 
entendimento pode ser obtido a partir da análise de sensibilidade. A sensibilidade em SEP pode auxiliar no entendimento das soluções, para determinar se uma solução viola seu limite após alguma perturbação, para estimar novas soluções depois de ocorridas pequenas perturbações no sistema entre outras aplicações.

Abaixo apresentaremos um histórico de alguns trabalhos em que a análise de sensibilidade foi empregada para auxiliar na obtenção de resultados.

PESCHON et al. (1968) foram uns dos pioneiros a aplicar o estudo de sensibilidade em sistema de energia elétrica. Foi mostrado que a sensibilidade, dentro do despacho ótimo, pode ser aplicada à vários casos diferentes, dos quais destacamos, 1) a obtenção da factibilidade nas equações de fluxos de potência através de interpretações correta nas variáveis duais; 2) a verificação da possibilidade de uma solução ótima melhorada violar alguma restrição do problema; 3) o aproveitamento da solução do problema de despacho ótimo, considerada trabalhosa, na obtenção de novas soluções somente com considerações de sensibilidade de segunda ordem. Os autores mostraram que através das equações de balanço de potência, podem definir as mudanças nas variáveis dependentes, quando alguma perturbação for imposta nas variáveis de independentes. Também são vistas as relações de sensibilidade com respeito as variáveis relacionadas a um problema de despacho ótimo de potência. Foi visto que, aplicando-se as condições de KKT ao problema de despacho ótimo em que se quer minimizar o custo $\mathrm{F}$, pode-se tirar várias conclusões: uma delas é que o vetor dual relacionado com as variáveis independentes mede a sensibilidade de F para um determinado controle; outra, é que o vetor dual 
relacionado com as equações dos fluxos mede o incremento do custo $\Delta \mathrm{F}$ para o sistema, quando há um incremento de demanda. Outro fator importante destacado é que quando analisamos as relações de sensibilidade com respeito as variáveis independentes, temos de ter as informações exatas do estado do sistema, pois um controle inadequado leva a custos inexatos de F. Foram feitas análises do desempenho do sistema de potência por meio das sensibilidades de primeira e segunda ordem, que levou à seguinte conclusão: sensibilidade de primeira ordem é relativamente fácil de ser calculada e pode ser usada como parte de um algoritmo para calcular o despacho ótimo; sensibilidade de segunda ordem, embora requeira cálculos mais elaborados, tem a capacidade de elaborar um tipo de lei de realimentação que mostra as pequenas mudanças na geração de potências ativas e reativas necessárias para manter a operação ótima quando ocorrerem pequenas alterações nas demandas. Uma possível desvantagem do método de sensibilidade de segunda ordem parece ser a inabilidade de trabalhar diretamente com restrições de desigualdade, que, em alguns problemas, são de muita importância; embora, na teoria, restrições de desigualdade possam ser consideradas adicionando-se uma função penalidade para o custo, como sugerido pelos autores.

KISHORE \& HILL (1971) desenvolveram um método que emprega análises de sensibilidade para minimizar a alocação de potência reativa, satisfazendo as restrições de operação. Neste método é empregada sensibilidade de primeira ordem, que relaciona as variáveis elétricas tensão e potência reativa. Foram aplicadas técnicas de programação linear para resolver o problema, incrementando na matriz sensibilidade os limites superiores e inferiores das tensões, fazendo com que esses limites sejam 
considerados. A injeção de reativos é minimizada na função objetivo. O problema de programação linear fica na forma padrão de programa linear, fazendo com que a solução do problema seja facilmente encontrada. Dois exemplos ilustram a aplicação do método.

GRIBIK et al. (1990) propuseram um método que utiliza análise de sensibilidade no problema de FPO. Os autores basearam-se no teorema da perturbação do ótimo, apresentado por FIACCO (1983), para desenvolverem uma formulação aplicada ao problema de FPO. Entretanto, eles não utilizaram o teorema na íntegra, pois somente as condições de otimalidade da função Lagrangiana foram consideradas para desenvolver a formulação. A formulação proposta não trabalha com as restrições de desigualdade. Os autores usaram o FPO proposto por SUN (1984) para encontrar o ponto ótimo a ser perturbado. Apresentaram, ainda, um teste em um sistema de 34 barras, no qual foi adicionado $1 \mathrm{MW}$ de carga em cada barra do sistema.

SMED (1993) apresentou um novo estudo de análise de sensibilidade. Nele a matriz sensibilidade resultante das equações do sistema de potência utilizada tem uma formulação esparsa. A formulação esparsa é obtida pela interpretação das variáveis que são calculadas no cálculo dos autovalores. Como resultado dessa modificação algum controle pode ser realizado com pequeno esforço computacional. A abordagem apresentada pelo autor mostra que quando realizamos uma mudança nos parâmetros do sistema, somente poucos elementos são afetados, o que não acontece com a sensibilidade dos autovalores tradicional. Essa nova análise de 
sensibilidade pode ser aplicada para vários casos relacionados com sistemas elétricos de potência. A metodologia foi aplicada em um sistema de potência real.

JAN \& CHEN (1995) desenvolveram um método que utiliza fatores de sensibilidade para análises de fluxo de potência. O método requer somente operações algébricas, sendo, assim, muito rápido e, portanto, podendo ter aplicação em tempo real. Depois de se resolver o problema do despacho ótimo através do método de Newton, as magnitudes das tensões podem exceder os valores padronizados. Os autores apresentaram dois passos que podem ser feitos para manter a magnitude da tensão das barras de carga dentro de seus limites. Passo 1: se alguma magnitude das tensões das barras de carga estão maiores que o limite máximo, usam-se fatores de sensibilidade de potência reativa para calcular o incremento nas barras de tensão controlada que possam fazer que a magnitude da tensão volte para dentro de seu limite. Passo 2: se alguma magnitude das tensões das barras de carga forem menores que o limite mínimo, usam-se o fatores de sensibilidade da potência reativa para calcular o capacitor a ser acoplado na barra de carga que possa alterar a magnitude da tensão para dentro de seu limite. Os autores validaram o método em um sistema de 245 barras.

GURRAN \& SUBRAMANYAN (1999) aplicaram o teorema Tellegen para a análise de sensibilidade. Uma derivação do teorema foi usada em uma rede de distribuição em um sistema radial de 400 volts e 12 barras, com um alimentador principal. A aplicação foi feita para perturbações de $10 \%$ e $20 \%$. As operações 
aritiméticas envolvidas e o tempo computacional foram pequenos, mostrando que a aplicação pode ser estendida a grandes sistemas.

FANG \& NGAN (2000) apresentaram um trabalho que contribui para estender o estudo em problemas de fluxo de potência. Os autores utilizaram um Unified Power Flow Controller (UPFC), que pode exercer controle independente nos fluxos das potências ativa e reativa das linhas de transmissão, regulagem da tensão, compensadores séries e shunts. Os UPFCs são vistos como um conjunto de Flexible AC Transmission Systems (FACTs), que são dispositivos baseados na eletrônica de potência e que podem proporcionar maior flexibilidade no controle e operação dos sistemas de potências. Nesse trabalho, as equações do Fluxo de carga são estendidas para serem analisadas juntamente com o UPFC, o que pode ser feito com pequenas alterações nas equações do fluxo de carga. São aplicadas técnicas de esparsidade, ordenamento ótimo e análise de sensibilidade. A análise de sensibilidade é usada para modelar os efeitos do UPFC no sistema, e os parâmetros do UPFC são usados como variáveis de controle. O método se mostrou robusto nos exemplos testados, podendo ser usado não somente para mostrar os efeitos do controle do UPFC embutido na linha, mas também para analisar os efeitos em outro ramo.

BELL \& KIRIRSCHEN (2000) apresentaram uma derivação da sensibilidade linear, usada no controle de despacho de MW, para auxílio nas violações das tensões. A análise de sensibilidade foi embutida com a idéia de se acrescentar uma ferramenta, para apoiar nas decisões de operações de sistema de potência. Os autores apresentaram três procedimentos que utiliza essas análises de sensibilidade na 
tentativa de resolver eventuais violações nos limites das tensões. O primeiro utiliza o controle da potência reativa para controlar as magnitudes de tensão, o segundo faz o controle de potência ativa para ajustar o fluxo de potência ativa nas linhas e o terceiro controla as magnitudes de tensão utilizando o controle da potência ativa. Os três procedimentos foram implementados em um algoritmo que utiliza conjunto fuzzy, o qual direciona o procedimento de controle utilizado para resolver os eventuais problemas. $\mathrm{O}$ controle exigido altera-se para cada sistema com referência a matriz de sensibilidade. Os controles são escolhidos equilibrando os critérios de baixo custo, preservação de margem de controle e simplicidade de implementação. A aplicação foi testada no sistema IEEE-RST.

AUMULLER et al. (2002) apresentaram um novo método de análises de instabilidade de tensão. O método é baseado no estudo da sensibilidade da geração de potência reativa em relação às variações de carga nas barras. A essência do método esta na utilização da técnica de análises de sensibilidade de primeira ordem. As informações obtidas pela análise de sensibilidade são usadas para identificar o conjunto de barras que causam instabilidade de tensão. O método foi testado em um sistema teste do Cigré e no "Qweensland Transmission System". 
Capítulo 3

\section{FLUXO DE POTÊNCIA ÓTIMO}

\subsection{INTRODUÇÃO}

Neste capítulo apresentaremos o desenvolvimento do método utilizado na resolução do problema de FPO e discutiremos sua implementação computacional. Esse método foi desenvolvido matematicamente por BAPTISTA (2001). Sua aplicação no problema de FPO é uma das contribuições desta pesquisa.

\subsection{O PROBLEMA DE FLUXO DE POTÊNCIA ÓTIMO.}

O FPO é um termo genérico que envolve uma classe de problemas, em que destacamos o problema de despacho ótimo de potência reativa, que apresentaremos nesta seção. O problema de FPO busca otimizar uma função específica, satisfazendo às restrições que são regidas por particularidades operacionais e físicas da rede elétrica. No problema de FPO, os modelos matemáticos utilizados envolvem dificuldades como não-linearidades, não-convexidades, milhares de restrições, 
variáveis discretas, o que ocasiona um problema de difícil solução; tal particularidade tem feito com que seja um tema abordado por vários pesquisadores.

O FPO pode ser modelado como o seguinte problema de programação nãolinear:

$$
\begin{aligned}
& \min f(x) \\
& \text { s.a } g(x)=0 \\
& h(x) \leq 0 \\
& x^{\min } \leq x \leq x^{\max }
\end{aligned}
$$

em que:

$\mathrm{x} \in \mathfrak{R}^{\mathrm{n}}$ : vetor das variáveis de estado;

$\mathrm{x}^{\min }$ e $\mathrm{x}^{\max }$ : limites superiores e inferiores das variáveis $\mathrm{x}$, respectivamente;

$\mathrm{f}(\mathrm{x})$ : função objetivo que representa o desempenho do sistema;

$\mathrm{g}(\mathrm{x}) \in \mathfrak{R}^{\mathrm{m}}$ : vetor das equações do fluxo de potência onde $\mathrm{m}<\mathrm{n}$;

$\mathrm{h}(\mathrm{x}) \in \mathfrak{R}^{\mathrm{r}}$ : vetor das restrições funcionais do fluxo de potência.

O vetor das variáveis de estado x representa a magnitude de tensão, $\mathrm{V}$, ângulos, $\theta$, e tap dos transformadores, t. A função objetivo, $\mathrm{f}(\mathrm{x})$, representa as perdas de potência ativa na transmissão. As restrições de igualdade , $\mathrm{g}(\mathrm{x})$, representam as equações de balanço de potência ativa e reativa nas barras da rede. As restrições de desigualdade $h(x)$ podem representar as potências reativa nas barras de controle de 
reativos, os fluxos ativos e reativos nas linhas de transmissão e o fluxo de intercâmbio.

Ao rescrevermos o problema (3.1) utilizando as equações de fluxo de potência como apresentada por MONTICELLI (1993), temos o seguinte problema.

$$
\begin{array}{lll}
\min & \sum_{\mathrm{m} \in \Omega \mathrm{k}} \mathrm{g}_{\mathrm{km}}\left(\mathrm{V}_{\mathrm{k}}^{2}+\mathrm{V}_{\mathrm{m}}^{2}-2 \mathrm{~V}_{\mathrm{k}} \mathrm{V}_{\mathrm{m}} \cos \theta_{\mathrm{km}}\right) & \\
\text { s.a. } & \mathrm{P}_{\mathrm{k}}-\sum_{\mathrm{m} \in \Omega \mathrm{k}} \mathrm{P}_{\mathrm{km}}=0 & \mathrm{k}=1, \cdots, \mathrm{NBCCR} \\
& \mathrm{Q}_{\mathrm{k}}+\mathrm{Q}_{\mathrm{k}}^{\mathrm{sh}}-\sum_{\mathrm{m} \in \Omega \mathrm{k}} \mathrm{Q}_{\mathrm{km}}=0 & \mathrm{k}=1, \cdots, \mathrm{NBC} \\
& \mathrm{Q}_{\mathrm{k}}^{\mathrm{min}}+\mathrm{Q}_{\mathrm{k}}^{\mathrm{sh}} \leq \sum_{\mathrm{m} \in \Omega \mathrm{k}} \mathrm{Q}_{\mathrm{km}} \leq \mathrm{Q}_{\mathrm{k}}^{\max }+\mathrm{Q}_{\mathrm{k}}^{\mathrm{sh}} & \mathrm{k}=1, \cdots, \mathrm{NBCR} \\
& \mathrm{V}_{\mathrm{k}}^{\min } \leq \mathrm{V}_{\mathrm{k}} \leq \mathrm{V}_{\mathrm{k}}^{\max } & \mathrm{k}=1, \cdots, \mathrm{NB} \\
& \mathrm{t}_{\mathrm{k}} \leq \mathrm{t}_{\mathrm{k}} \leq \mathrm{t}_{\mathrm{k}} & \mathrm{k}=1, \cdots, \mathrm{NT}
\end{array}
$$

O problema apresentado é conhecido como despacho ótimo de reativo (DOR) ou, simplesmente, FPO reativo. O aspecto reativo em um sistema de energia está relacionado principalmente ao controle da tensão nas barras. No DOR, os controles ativos são fixados, e as variáveis de controle relacionadas à potência reativa são otimizadas em relação à função objetivo. As variáveis de controle incluem geração de potência reativa, magnitude de tensão nas barras de geração, compensação síncrona, tap de transformador e admitância shunt, que pode ser capacitiva ou indutiva. As variáveis dependentes incluem ângulo de tensão, magnitude de tensão em barras de carga e fluxo nas linhas de transmissão. A função objetivo corresponde as perdas de potência ativa na transmissão, o que pode dificultar a resolução do 
problema de FPO segundo MONTICELLI \& LIU (1992), por ser não convexa e não separável.

Encontram-se, na literatura, diversas publicações que enfocam a formulação e a solução do problema de FPO. Destacam-se dentre esses trabalhos, diferentes métodos, abordados por vários pesquisadores. No entanto, todos estes métodos apresentam vantagens e desvantagens quanto à eficiência computacional e à flexibilidade no tratamento dos aspectos operacionais do problema. A abordagem que utilizaremos, na resolução do problema, é composta por uma metodologia mista. As variáveis canalizadas serão tratadas por uma função barreira logarítmica; as restrições de igualdade, pelo método de Newton; e as desigualdades, pela função Lagrangiana Aumentada. O objetivo desta metodologia mista é utilizar as melhores características de cada método.

\subsection{METODOLOGIA DE RESOLUÇÃO APLICADA AO PROBLEMA DE FPO}

Vários métodos podem ser utilizados na resolução do problema de FPO. Entre eles podemos destacar o método de Newton, da Lagrangiana aumentada e da barreira logarítmica. O método de Newton apresenta um excelente desempenho para problemas com restrições de igualdade. Contudo, para restrições de desigualdade, exige-se que se conheça o conjunto ativo das restrições na solução. O método da Lagrangiana aumentada apresenta um melhor comportamento em relação ao método das penalidades, pois ameniza o problema de mal condicionamento da matriz Hessiana. Finalmente, no método da barreira logarítmica, gerenciar o fator de 
barreira e seu parâmetro, garantindo a convergência do método, é uma tarefa difícil, no entanto, sua aplicação em problemas com restrições canalizadas é de fácil implementação.

Com o objetivo de aproveitar as melhores características destes métodos, será utilizada uma abordagem do método da função Lagrangiana aumentada para resolver o problema (3.1). Essa abordagem é denominada Método da Função Lagrangiana Aumentada-Barreira Logarítmica, BAPTISTA (2001). O método proposto trata as restrições canalizadas através do método de barreira logarítmica - incorporadas à função objetivo - resultando em um novo problema, que é associado à função Lagrangiana aumentada proposta por COSTA (1990). As condições necessárias de primeira ordem são aplicadas resultando num sistema não linear, que é resolvido pelo método de Newton. A solução através do método de Newton fornece os fatores de correção para atualizarmos os multiplicadores de Lagrange associados às igualdades, e ao vetor x e às variáveis de folga. Os multiplicadores de Lagrange associados às desigualdades são determinados utilizando-se a regra de atualização da função Lagrangiana aumentada proposta por HESTENES (1969), e os fatores de penalidades e barreira são atualizados por um fator heurístico.

\subsection{A FUNÇÃO LAGRANGIANA AUMENTADA-BARREIRA LOGARÍTMICA}

Nesta seção apresentaremos a função Lagrangiana aumentada-barreira logarítmica, baseada nos conceitos dos métodos de barreira e da Lagrangiana aumentada. 
Dado o problema (3.1), acrescentamos as variáveis de excesso e de folga como apresentado em (3.3).

$\min f(x)$

s.a. $g_{i}(x)=0, i=1, \ldots, m$

$\mathrm{h}_{\mathrm{j}}(\mathrm{x})+\mathrm{z}_{\mathrm{j}}=0, \mathrm{j}=1, \ldots, \mathrm{r}$

$\mathrm{x}+\mathrm{su}=\mathrm{x}^{\max }$

$\mathrm{x}-\mathrm{si}=\mathrm{x}^{\mathrm{min}}$

$\mathrm{z}_{\mathrm{j}} \geq 0, \mathrm{j}=1, \ldots, \mathrm{r}$

$\mathrm{su} \geq 0$

si $\geq 0$

onde $\quad(s u)^{t}=\left(s_{1}, \ldots, s u_{n}\right), \quad c o m \quad s u_{k} \geq 0, \quad$ e $\quad(s i)^{t}=\left(s_{1}, \ldots, s_{n}\right) \quad$ com $\quad s i_{k} \geq 0$, $\mathrm{k}=1, \cdots, \mathrm{n}$. As variáveis $\mathrm{z}_{\mathrm{j}}, \mathrm{j}=1, \cdots, \mathrm{r}$, bem como as componentes do vetor su são variáveis de folga, e as componentes do vetor si são variáveis de excesso. Denominamos su e si de vetores auxiliares.

O problema (3.3) pode ser transformado no seguinte problema equivalente:

$$
\begin{array}{ll}
\min & \mathrm{f}(\mathrm{x})-\delta\left(\sum_{\mathrm{k}=1}^{\mathrm{n}} \ln \mathrm{su}_{\mathrm{k}}+\sum_{\mathrm{k}=1}^{\mathrm{n}} \ln \mathrm{si}_{\mathrm{k}}\right) \\
\text { s.a } & \mathrm{g}_{\mathrm{i}}(\mathrm{x})=0, \mathrm{i}=1, \ldots, \mathrm{m} \\
& \mathrm{h}_{\mathrm{j}}(\mathrm{x})+\mathrm{z}_{\mathrm{j}}=0, \mathrm{j}=1, \ldots, \mathrm{r} \\
& \mathrm{x}+\mathrm{su}=\mathrm{x}^{\max } \\
& \mathrm{x}-\mathrm{si}=\mathrm{x}^{\min } \\
& \mathrm{z}_{\mathrm{j}} \geq 0, \mathrm{j}=1, \ldots, \mathrm{r}
\end{array}
$$

onde $\delta$ é o fator de barreira. 
Adicionamos à função objetivo a condição de não negatividade, através da função barreira logarítmica. O problema modificado (3.4) tende à mesma solução que o problema original (3.1).

Associamos ao problema (3.4) a seguinte função Lagrangiana aumentada:

$$
\begin{aligned}
& \mathrm{La}(\mathrm{x}, \mathrm{s}, \lambda, \pi, \mu, \mathrm{z})=\mathrm{f}(\mathrm{x})-\delta\left(\sum_{\mathrm{k}=1}^{\mathrm{n}} \ln \mathrm{su}_{\mathrm{k}}+\sum_{\mathrm{k}=1}^{\mathrm{n}} \ln \mathrm{si} \mathrm{i}_{\mathrm{k}}\right)+\frac{1}{2} \mathrm{c} \sum_{\mathrm{j}=1}^{\mathrm{r}}\left(\mathrm{h}_{\mathrm{j}}(\mathrm{x})+\mathrm{z}_{\mathrm{j}}\right)^{2}+\sum_{\mathrm{i}=1}^{\mathrm{m}} \lambda_{\mathrm{i}} \mathrm{g}_{\mathrm{i}}(\mathrm{x})+ \\
& \sum_{\mathrm{k}=1}^{\mathrm{n}} \pi \mathrm{u}_{\mathrm{k}}\left(\mathrm{x}_{\mathrm{k}}+\mathrm{su}_{\mathrm{k}}-\mathrm{x}_{\mathrm{k}}^{\max }\right)+\sum_{\mathrm{k}=1}^{\mathrm{n}} \pi \mathrm{i}_{\mathrm{k}}\left(\mathrm{x}_{\mathrm{k}}-\mathrm{si}_{\mathrm{k}}-\mathrm{x}_{\mathrm{k}}^{\min }\right)+\sum_{\mathrm{j}=1}^{\mathrm{r}} \mu_{\mathrm{j}}\left(\mathrm{h}_{\mathrm{j}}(\mathrm{x})+\mathrm{z}_{\mathrm{j}}\right)
\end{aligned}
$$

onde $\lambda, \pi \mathrm{u}, \pi \mathrm{i}$ e $\mu$ são os vetores dos multiplicadores de Lagrange, $\mathrm{c}>0$ é o fator de penalidade, e $\delta$, o fator de barreira.

Aplicando as condições necessárias de primeira ordem à função Lagrangiana (3.5) em relação a $z_{j}$ temos:

$\nabla_{z_{j}} \operatorname{La}(x, s, \lambda, \pi, \mu, z)=0, j=1, \ldots, r$

ou,

$$
z_{j}=-\frac{\mu_{j}}{c}-h_{j}(x), j=1, \ldots, r
$$

Como $\mathrm{z}_{\mathrm{j}} \geq 0$, temos: 


$$
z_{j}=\left\{\begin{array}{c}
-\frac{\mu_{j}}{c}-h_{j}(x), s e-\frac{\mu_{j}}{c}-h_{j}(x) \geq 0 \\
0, \text { se }-\frac{\mu_{j}}{c}-h_{j}(x) \leq 0
\end{array} j=1, \ldots, r .\right.
$$

Substituindo a eq.(3.8) em (3.5), obtemos a função Lagrangiana aumentadabarreira logarítmica:

$$
\begin{gathered}
\operatorname{La}(\mathrm{x}, \mathrm{s}, \lambda, \pi, \mu)=\mathrm{f}(\mathrm{x})-\delta\left(\sum_{\mathrm{k}=1}^{\mathrm{n}} \ln \mathrm{su}_{\mathrm{k}}+\sum_{\mathrm{k}=1}^{\mathrm{n}} \ln s \mathrm{i}_{\mathrm{k}}\right)+\sum_{\mathrm{i}=1}^{\mathrm{m}} \lambda_{\mathrm{i}} \mathrm{g}_{\mathrm{i}}(\mathrm{x})+\sum_{\mathrm{l}=1}^{\mathrm{n}} \pi \mathrm{u}_{\mathrm{k}}\left(\mathrm{x}_{\mathrm{k}}+\mathrm{su}_{\mathrm{k}}-\mathrm{x}_{\mathrm{k}}^{\max }\right)+ \\
\sum_{\mathrm{l}=1}^{\mathrm{n}} \pi \mathrm{i}_{\mathrm{k}}\left(\mathrm{x}_{\mathrm{k}}-\mathrm{si}_{\mathrm{k}}-\mathrm{x}_{\mathrm{k}}^{\operatorname{mim}}\right)+\sum_{\mathrm{j}=1}^{\mathrm{r}}\left\{\begin{array}{c}
\mu_{\mathrm{j}} \mathrm{h}_{\mathrm{j}}(\mathrm{x})+\frac{1}{2} \mathrm{ch}_{\mathrm{j}}^{2}(\mathrm{x}), \mathrm{se} \mathrm{h}_{\mathrm{j}}(\mathrm{x}) \geq-\frac{\mu_{\mathrm{j}}}{\mathrm{c}} \\
-\frac{\mu_{\mathrm{j}}^{2}}{2 \mathrm{c}}, \text { se } \mathrm{h}_{\mathrm{j}}(\mathrm{x}) \leq-\frac{\mu_{\mathrm{j}}}{\mathrm{c}}
\end{array}\right.
\end{gathered}
$$

Definimos, deste modo, a função Lagrangiana aumentada-barreira logarítmica associada ao problema (3.1).

\subsection{O MÉTODO DA FUNÇÃO LAGRANGIANA AUMENTADA- BARREIRA LOGARÍTMICA}

Apresentaremos, nesta seção, o método da função Lagrangiana aumentadabarreira logarítmica, para a resolução do problema (3.1). A solução do problema (3.1) é encontrada através da resolução de uma seqüência de problemas irrestritos. Aplicando as condições de otimalidade sobre a função Lagrangiana aumentadabarreira logarítmica (3.9), obtemos um sistema não linear, cuja solução são as direções de busca, $\Delta \mathrm{x}, \Delta \mathrm{su}, \Delta \mathrm{si}, \Delta \lambda, \Delta \pi \mathrm{u}$ e $\Delta \pi \mathrm{i}$, como segue: 


$$
\left\{\begin{array}{l}
\nabla_{\mathrm{x}} \operatorname{La}(\mathrm{x}, \mathrm{s}, \lambda, \pi, \mu)=0 \\
\nabla_{\mathrm{su}} \operatorname{La}(\mathrm{x}, \mathrm{s}, \lambda, \pi, \mu)=0 \\
\nabla_{\mathrm{si}} \operatorname{La}(\mathrm{x}, \mathrm{s}, \lambda, \pi, \mu)=0 \\
\nabla_{\lambda} \operatorname{La}(\mathrm{x}, \mathrm{s}, \lambda, \pi, \mu)=0 \\
\nabla_{\pi \mathrm{u}} \operatorname{La}(\mathrm{x}, \mathrm{s}, \lambda, \pi, \mu)=0 \\
\nabla_{\pi \mathrm{i}} \operatorname{La}(\mathrm{x}, \mathrm{s}, \lambda, \pi, \mu)=0
\end{array}\right.
$$

ou, equivalentemente,

$$
\begin{aligned}
& \nabla_{x} f(x)^{t}+\lambda^{t} J(x)+(\pi u)^{t} I+(\pi i)^{t} I+\sum_{j=1}^{r}\left\{\begin{array}{c}
\left(\mu_{j}+\operatorname{ch}_{j}(x)\right) \nabla_{x} h_{j}(x)^{t}, \operatorname{seh}_{j}(x) \geq-\frac{\mu_{j}}{c} \\
0, \text { se } h_{j}(x) \leq-\frac{\mu_{j}}{c}
\end{array}=0\right. \\
& -\frac{\delta}{\mathrm{su}_{\mathrm{k}}}+\pi \mathrm{u}_{\mathrm{k}}=0, \mathrm{k}=1, \ldots, \mathrm{n} \\
& -\frac{\delta}{\mathrm{si}_{\mathrm{k}}}-\pi \mathrm{i}_{\mathrm{k}}=0, \mathrm{k}=1, \ldots, \mathrm{n} \\
& \mathrm{g}_{\mathrm{i}}(\mathrm{x})=0, \mathrm{i}=1, \ldots, \mathrm{m} \\
& \mathrm{x}+\mathrm{su}-\mathrm{x}^{\max }=0 \\
& \mathrm{x}-\mathrm{si}-\mathrm{x}^{\min }=0
\end{aligned}
$$

onde $\mathrm{J}(\mathrm{x})^{\mathrm{t}}=\left(\nabla_{\mathrm{x}} \mathrm{g}_{1}(\mathrm{x}), \ldots, \nabla_{\mathrm{x}} \mathrm{g}_{\mathrm{m}}(\mathrm{x})\right)$ é denominada matriz Jacobiana, a mesma do fluxo de potência, I uma matriz identidade.

Obtemos a solução do sistema não linear (3.11) utilizando o método de Newton-Raphson, o que resulta no sistema matricial, que, em sua forma simplificada, é representada por:

$$
\mathrm{W} \Delta \mathrm{d}=-\nabla \mathrm{La}
$$


onde,

$$
\mathrm{W}=\left(\begin{array}{cccccc}
\nabla_{\mathrm{xx}}^{2} \mathrm{La} & 0 & 0 & \mathrm{~J}(\mathrm{x})^{\mathrm{t}} & \mathrm{I} & \mathrm{I} \\
0 & \delta(\mathrm{Su}) & 0 & 0 & \mathrm{I} & 0 \\
0 & 0 & \delta(\mathrm{Si}) & 0 & 0 & -\mathrm{I} \\
\mathrm{J}(\mathrm{x}) & 0 & 0 & 0 & 0 & 0 \\
\mathrm{I} & \mathrm{I} & 0 & 0 & 0 & 0 \\
\mathrm{I} & 0 & -\mathrm{I} & 0 & 0 & 0
\end{array}\right)
$$

é a matriz Lagrangiana, com

$$
\mathrm{Su}=\left(\begin{array}{ccc}
\frac{1}{\left(\mathrm{su}_{1}\right)^{2}} & & 0 \\
0 & \ddots & \frac{1}{\left(\mathrm{su}_{\mathrm{n}}\right)^{2}}
\end{array}\right), \quad \mathrm{Si}=\left(\begin{array}{ccc}
\frac{1}{\left(\mathrm{si}_{1}\right)^{2}} & \ddots & 0 \\
0 & \frac{1}{\left(\mathrm{si}_{\mathrm{n}}\right)^{2}}
\end{array}\right)
$$

$\mathrm{e}$

$\nabla_{x x}^{2} L a=\nabla_{x x}^{2} f(x)+\sum_{i=1}^{m} \lambda_{i} \nabla_{x x}^{2} g_{i}(x)+\sum_{j=1}^{r}\left\{\begin{array}{c}\left(\mu_{j}+c_{j}(x)\right) \nabla_{x x}^{2} h_{j}(x)+c\left(\nabla_{x} h_{j}(x)\right) \nabla_{x} h_{j}(x)^{t}, s e h_{j}(x) \geq-\frac{\mu_{j}}{c} \\ 0, s e h_{j}(x) \leq-\frac{\mu_{j}}{c}\end{array}\right.$

$\Delta \mathrm{d}^{\mathrm{t}}=(\Delta \mathrm{x}, \Delta \mathrm{su}, \Delta \mathrm{si}, \Delta \lambda, \Delta \pi \mathrm{u}, \Delta \pi \mathrm{i})$ é o vetor das direções de busca, e 


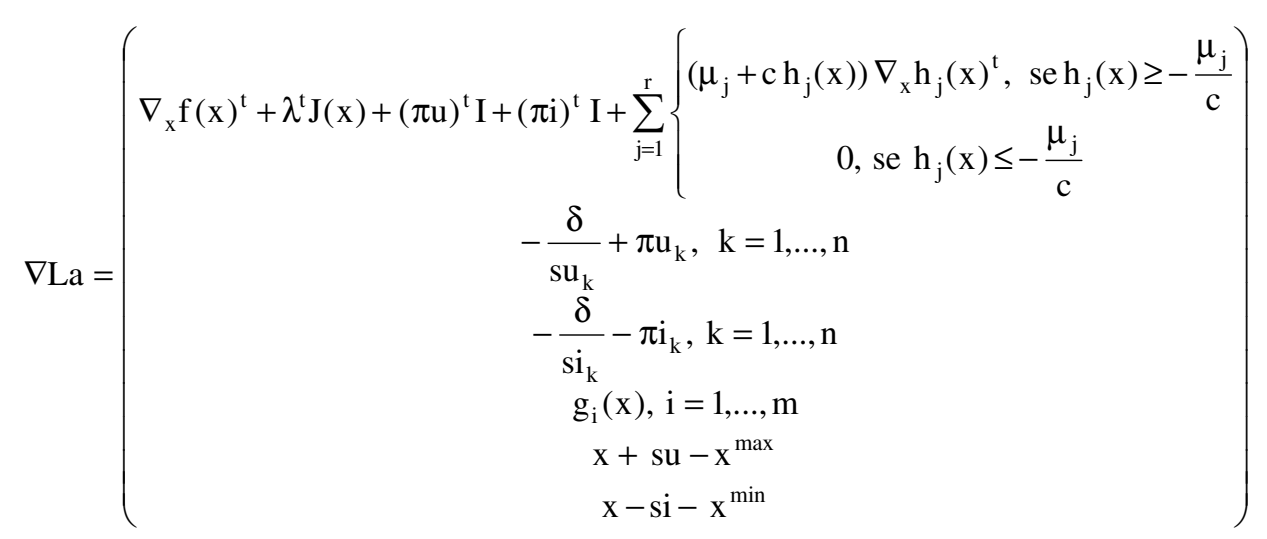

é denominado vetor gradiente.

A solução do sistema (3.12) fornece os incrementos das variáveis, $\Delta_{\mathrm{s}}$, utilizados na atualização das mesmas. As variáveis $x$, su e si, e os vetores multiplicadores de Lagrange, $\lambda, \pi \mathrm{u}$ e $\pi \mathrm{i}$ são atualizados da seguinte forma:

$$
\begin{aligned}
& \mathrm{x}^{\mathrm{k}+1}=\mathrm{x}^{\mathrm{k}}+\alpha_{\mathrm{p}} \Delta \mathrm{x}^{\mathrm{k}} \\
& \mathrm{su}^{\mathrm{k}+1}=\mathrm{su}^{\mathrm{k}}+\alpha_{\mathrm{p}} \Delta \mathrm{su}^{\mathrm{k}} \\
& \mathrm{si}^{\mathrm{k}+1}=\mathrm{si}^{\mathrm{k}}+\alpha_{\mathrm{p}} \Delta \mathrm{si}^{\mathrm{k}} \\
& \lambda^{\mathrm{k}+1}=\lambda^{\mathrm{k}}+\alpha_{\mathrm{d}} \Delta \lambda^{\mathrm{k}} \\
& \pi \mathrm{u}^{\mathrm{k}+1}=\pi \mathrm{u}^{\mathrm{k}}+\alpha_{\mathrm{d}} \Delta \pi \mathrm{u}^{\mathrm{k}} \\
& \pi \mathrm{i}^{\mathrm{k}+1}=\pi \mathrm{i}^{\mathrm{k}}+\alpha_{\mathrm{d}} \Delta \pi \mathrm{i}^{\mathrm{k}}
\end{aligned}
$$

onde $\alpha_{\mathrm{p}}$ e $\alpha_{\mathrm{d}}$ são os passos utilizados na atualização das variáveis primais e duais, respectivamente. 
Esses passos são calculados de forma que cada componente dos vetores das variáveis auxiliares su e si permaneça estritamente positiva, e os elementos dos vetores duais $\lambda, \pi \mathrm{u} \pi \mathrm{i}$ permaneçam com seus respectivos sinais. Uma sugestão para o cálculo do passo máximo é a estratégia utilizada por GRANVILLE (1994) e QUINTANA (1995), dada por:

$$
\begin{aligned}
& \alpha_{\mathrm{p}}=\min \left\{\sigma\left[\left(\min _{\Delta \mathrm{su}<0} \frac{\mathrm{su}}{|\Delta \mathrm{su}|}, \min _{\Delta \mathrm{si}<0} \frac{\mathrm{si}}{|\Delta \mathrm{si}|}\right), 1\right]\right\} \\
& \alpha_{\mathrm{d}}=\min \left\{\sigma\left[\left(\min _{\Delta \pi \mathrm{u}>0} \frac{-\pi \mathrm{u}}{|\Delta \pi \mathrm{u}|}, \min _{\Delta \pi \mathrm{i}<0} \frac{\pi \mathrm{i}}{|\Delta \pi \mathrm{i}|}\right), 1\right]\right\}
\end{aligned}
$$

onde $\sigma=0.9995$ é um valor determinado empiricamente e que, segundo WRIGHT (1995), é derivada da fórmula $1-\frac{1}{9 \sqrt{\mathrm{m}}}$, onde $\mathrm{m}$ é o número de restrições do problema.

Os multiplicadores de Lagrange $\mu$ são atualizados utilizando-se a regra de HESTENES (1969):

$$
\mu_{j}^{k+1}=\left\{\begin{array}{c}
\mu_{j}^{k}+c^{k} h_{j}\left(x^{k+1}\right), \text { se } h_{j}\left(x^{k+1}\right) \geq-\frac{\mu_{j}^{k}}{c^{k}} j=1, \ldots, r \\
0, \text { se } h_{j}\left(x^{k+1}\right) \leq-\frac{\mu_{j}^{k}}{c^{k}}
\end{array}\right.
$$

e o fator de penalidade c e o fator de barreira $\delta$, respectivamente, da seguinte forma: 


$$
\begin{aligned}
& c^{\mathrm{k}+1}=\beta c^{\mathrm{k}}, \beta>1 \\
& \delta^{\mathrm{k}+1}=\frac{\delta^{\mathrm{k}}}{\rho}, \rho>1 .
\end{aligned}
$$

onde $\beta$ e $\rho$ são denominados fatores de correção.

\subsection{ALGORITMO}

A nova abordagem do método da função Lagrangiana aumentada-barreira pode ser apresentada pelo seguinte algoritmo:

\section{Passo inicial}

Dado o problema (3.1) construa a Função Lagrangiana aumentada-barreira logarítmica (3.9);

Faça $\mathrm{k}=0$;

Escolha uma solução inicial para as variáveis do problema: $\mathrm{x}^{0}, \lambda^{0},(\pi \mathrm{u})^{0}$, $(\pi \mathrm{i})^{0},(\mathrm{su})^{0},(\mathrm{si})^{0}, \mu^{0}, \mathrm{c}^{0}, \delta^{0}$

\section{Passo iterativo}

I1) Determine o sistema (3.12) e resolva-o;

I2) Atualize as variáveis $\mathrm{x}, \lambda$, su, si, $\pi \mathrm{u}$ e $\pi \mathrm{i}$ utilizando (3.13);

I3) Se o critério de parada escolhido para o método de Newton está satisfeito, vá ao passo I4;

Senão, volte ao passo I1; 
I4) Se as condições de KKT estão satisfeitas, FIM; Senão, vá ao passo I5;

I5) Atualize os multiplicadores de Lagrange e os fatores de penalidade e barreira usando (3.16) e (3.17), k= k+1 e volte a I1.

A técnica preserva as características do método da função Lagrangiana aumentada, pois caminha pelo exterior da região factível, porém, obedece às restrições de canalização. A convergência do algoritmo está diretamente ligada à escolha dos fatores iniciais de penalidade e barreira e de seus parâmetros de correção.

Em razão do processo de linearização é gerado um sistema matricial esparso. A seguir apresentaremos a técnica de esparsidade utilizada na resolução do sistema linearizado.

\subsection{TÉCNICAS DE ESPARSIDADE}

A resolução do sistema (3.12) envolve o manuseio de um sistema algébrico linear de grande dimensão com uma matriz de coeficiente altamente esparsa. A eficiência na resolução deste sistema depende do modelo e da técnica de esparsidade utilizadas.

Há várias publicações e estudos destes métodos, entre eles podemos destacar a eliminação de Gauss, NOBUO \& TINNEY (1963), TINNEY \& WALKER (1967), ZOLLENKOPF (1971), EISENSTAT et al. (1981) e TINNEY et al. (1985). Também 
há o pacote de sub-rotinas desenvolvidas por L.S Duff e J.K. Reid, do Rutherford Appleton Laboratory.

Entre os métodos vistos, optamos por utilizar a sub-rotina, M57 desenvolvida por L.S Duff e J.K. Reid, do Rutherford Appleton Laboratory. Essa sub-rotina determina a solução de sistemas lineares esparsos, utilizando uma variante da eliminação de Gauss.

Considerando as características da matriz gerada na solução do FPO, simétrica de valor e de posição, não existe a necessidade de se trabalhar com todos os valores da matriz. Pode-se usar apenas a matriz triangular superior ou inferior. A sub-rotina MA57 é ideal para a aplicação neste algoritmo, pois necessita apenas dos valores da matriz triangular superior ou inferior para a solução do problema. Com isso, economiza-se memória e tempo de processamento, uma vez que o número de elementos a serem armazenados é bem menor.

\subsection{IMPLEMENTAÇÃO COMPUTACIONAL}

A implementação computacional do programa de FPO foi realizada em linguagem FORTRAN; o programa foi desenvolvido em um micro-computador Pentium III - $800 \mathrm{MHz}$, com 256 Mbytes de memória RAM, do Laboratório de Otimização de Sistemas Elétricos de Potência (LOSEP), do Departamento de Engenharia Elétrica da Escola de Engenharia de São Carlos (EESC - USP). Na implementação utilizamos a dupla precisão aritmética. 
O programa foi dividido nos seguintes módulos, mostrados na Figura 3.1:

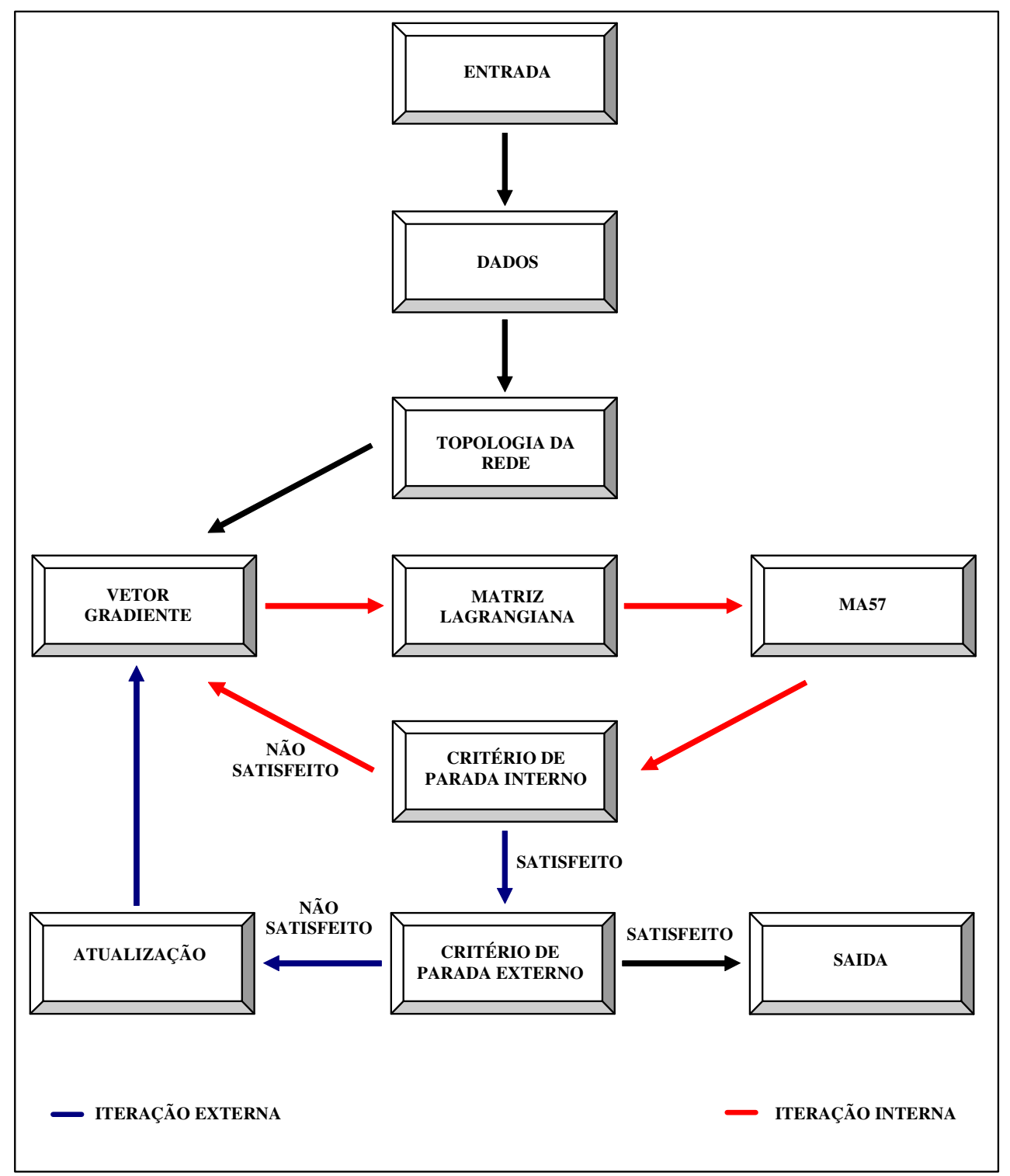

Figura 3.1 - Módulos do programa do Método da Função Lagrangiana AumentadaBarreira Logarítmica, aplicado ao problema de FPO

em que:

ENTRADA - faz a declaração das variáveis e constantes utilizadas no programa (variáveis globais); 
DADOS - faz a leitura dos dados do sistema, isto é, os dados de barras e dados de linhas;

TOPOLOGIA DA REDE - armazena a topologia do sistema elétrico, isto é, as ligações existentes entre as barras. É de fundamental importância para a criação do vetor gradiente e da matriz Lagrangiana;

VETOR GRADIENTE - constrói o vetor gradiente associado à função Lagrangiana aumentada-barreira logarítmica;

MATRIZ LAGRANGIANA - constrói a matriz Lagrangiana, W, associada à função Lagrangiana aumentada-barreira logarítmica. A matriz W é utilizada na resolução do sistema linear (3.12);

MA57 - resolve o sistema linear (3.12) utilizando a sub-rotina MA57 até a satisfação de um critério de parada interno;

CRITÉRIO DE PARADA INTERNO - verifica se as restrições de igualdade foram satisfeitas dentro de uma tolerância predeterminada;

CRITÉRIO DE PARADA EXTERNO - verifica se as condições de KKT foram satisfeitas;

ATUALIZAÇÃO - atualiza os fatores de penalidade e barreira por um parâmetro predeterminado; 
SAÍDA - gera o arquivo de saída que contém os dados do sistema otimizado, variáveis primais e duais.

O programa é composto de iterações denominadas internas e externas. As iterações internas correspondem à resolução sucessiva do sistema linear (3.12) com a utilização da sub-rotina MA57 até a satisfação de um critério de parada interno; as iterações externas correspondem à atualização dos fatores de penalidade e barreira até a satisfação de um critério de parada externo. 


\section{Capítulo 4}

\section{ANÁLISE DE SENSIBILIDADE}

\subsection{INTRODUÇÃO}

$\mathrm{Na}$ literatura especializada, há muitas citações do uso de análise de sensibilidade, tanto em problemas de programação linear (PL), como em problemas de programação não linear (PNL). Neste capítulo apresentaremos uma metodologia de análise de sensibilidade voltada para problemas de PNL, que será empregada no próximo capítulo ao problema de FPO.

A metodologia apresentada é baseada no teorema proposto por FIACCO (1976). O teorema utiliza a análise de sensibilidade de primeira ordem aplicada à solução local de segunda ordem, para estimar a solução após a ocorrência de perturbações no problema de PNL. As perturbações podem ser tanto nas restrições como na função objetivo. A análise de sensibilidade que apresentaremos considera apenas as perturbações nas restrições de igualdade. 


\subsection{FORMULAÇÃO MATEMÁTICA DA SENSIBILIDADE UTILIZADA}

Ao problema de PNL associaremos perturbações, $\varepsilon$, nas restrições de igualdade.

$\min f(x)$

$$
\begin{array}{lll}
\text { s.a } & \mathrm{g}_{\mathrm{i}}(\mathrm{x})+\varepsilon_{\mathrm{i}}=0, \quad \mathrm{i}=1, \ldots, \mathrm{m} \\
& \mathrm{h}_{\mathrm{j}}(\mathrm{x}) \leq 0, \quad \mathrm{j}=1, \ldots, \mathrm{r} \\
& \mathrm{x}^{\min } \leq \mathrm{x} \leq \mathrm{x}^{\max } &
\end{array}
$$

em que:

$\varepsilon=\left(\varepsilon_{1}, \ldots, \varepsilon_{\mathrm{m}}\right)$ - vetor perturbação.

Para encontrarmos a solução para o problema, depois de realizadas perturbações, isto é, $\varepsilon \neq 0$, associamos a seguinte função Lagrangiana:

$\mathrm{L}(\mathrm{x}, \mu, \lambda, \varepsilon)=\mathrm{f}(\mathrm{x})+\sum_{\mathrm{i}=1}^{\mathrm{m}} \lambda_{\mathrm{i}}\left[\mathrm{g}_{\mathrm{i}}(\mathrm{x})+\varepsilon_{\mathrm{i}}\right]+\sum_{\mathrm{j}=1}^{\mathrm{r}} \mu_{\mathrm{j}} \mathrm{h}_{\mathrm{j}}(\mathrm{x})$

onde $\lambda$ é o vetor dos multiplicadores de Lagrange associados às restrições de igualdade, e $\mu$ é o vetor dos multiplicadores de Lagrange para as restrições de desigualdade ativas. 
Para aplicarmos a técnica de sensibilidade, é preciso termos primeiramente a solução ótima para o problema, $x^{*}, \lambda^{*}$ e $\mu^{*}$, sem perturbação, ou seja, a solução para $\varepsilon=0$.

A técnica de sensibilidade considera o gradiente da função Lagrangiana, a folga complementar e as restrições de igualdade perturbada, isto é:

$\nabla_{\mathrm{x}} \mathrm{L}(\mathrm{x}, \mu, \lambda, \varepsilon)=0$

$\mu_{\mathrm{j}}\left[\mathrm{h}_{\mathrm{j}}(\mathrm{x})\right]=0 \quad \mathrm{j}=1, \ldots, \mathrm{r}$

$\mathrm{g}_{\mathrm{i}}(\mathrm{x})+\varepsilon_{\mathrm{i}}=0 \quad \mathrm{i}=1, \ldots, \mathrm{m}$

onde, $\mu \geq 0, \lambda$ irrestrito e o gradiente da função Lagrangiana representado por:

$\nabla_{\mathrm{x}} \mathrm{L}(\mathrm{x}, \mu, \lambda, \varepsilon)=\nabla_{\mathrm{x}} \mathrm{f}(\mathrm{x})+\sum_{\mathrm{i}=1}^{\mathrm{m}} \lambda_{\mathrm{i}} \nabla_{\mathrm{x}}\left[\mathrm{g}_{\mathrm{i}}(\mathrm{x})+\varepsilon_{\mathrm{i}}\right]+\sum_{\mathrm{j}=1}^{\mathrm{r}} \mu_{\mathrm{j}} \nabla_{\mathrm{x}} \mathrm{h}_{\mathrm{j}}(\mathrm{x})$

Determinamos as raízes do sistema não linear (4.3), linearizando o sistema no ponto $\left(\mathrm{x}^{*}, \lambda^{*}, \mu^{*}\right)$, o que resulta no seguinte sistema linear:

$$
\begin{array}{lr}
\nabla_{\mathrm{x}} \mathrm{L}\left(\mathrm{x}^{*}, \mu^{*}, \lambda^{*}, \varepsilon\right)+\nabla_{\mathrm{xx}} \mathrm{L}\left(\mathrm{x}^{*}, \mu^{*}, \lambda^{*}, \varepsilon\right) \Delta \mathrm{x}+\nabla_{\mathrm{x} \mu} \mathrm{L}\left(\mathrm{x}^{*}, \mu^{*}, \lambda^{*}, \varepsilon\right) \Delta \mu+\nabla_{\mathrm{x} \lambda} \mathrm{L}\left(\mathrm{x}^{*}, \mu^{*}, \lambda^{*}, \varepsilon\right) \Delta \lambda & =0 \\
\mu_{\mathrm{j}}^{*} \mathrm{~h}_{\mathrm{j}}\left(\mathrm{x}^{*}\right)+\mu_{\mathrm{j}}^{*} \nabla_{\mathrm{x}} \mathrm{h}_{\mathrm{j}}\left(\mathrm{x}^{*}\right) \Delta \mathrm{x}+\nabla_{\mu} \mu_{\mathrm{j}}^{*} \mathrm{~h}_{\mathrm{j}}\left(\mathrm{x}^{*}\right) \Delta \mu & =0 \\
{\left[\mathrm{~g}_{\mathrm{i}}\left(\mathrm{x}^{*}\right)+\varepsilon_{\mathrm{i}}\right]+\nabla_{\mathrm{x}}\left[\mathrm{g}_{\mathrm{i}}\left(\mathrm{x}^{*}\right)+\varepsilon_{\mathrm{i}}\right] \Delta \mathrm{x}} & =0
\end{array}
$$


Eliminando os termos nulos temos:

$$
\begin{array}{ll}
\nabla_{\mathrm{xx}} \mathrm{L}\left(\mathrm{x}^{*}, \mu^{*}, \lambda^{*}, \varepsilon\right) \Delta \mathrm{x}+\nabla_{\mathrm{x}} \mathrm{h}_{\mathrm{j}}\left(\mathrm{x}^{*}\right) \Delta \mu+\nabla_{\mathrm{x}} \mathrm{g}_{\mathrm{i}}\left(\mathrm{x}^{*}\right) \Delta \lambda & =0 \\
\mu_{\mathrm{j}}^{*} \nabla_{\mathrm{x}} \mathrm{h}_{\mathrm{j}}\left(\mathrm{x}^{*}\right) \Delta \mathrm{x}+\mathrm{h}_{\mathrm{j}}\left(\mathrm{x}^{*}\right) \Delta \mu & =0 \\
\varepsilon_{\mathrm{i}}+\nabla_{\mathrm{x}} \mathrm{g}_{\mathrm{i}}\left(\mathrm{x}^{*}\right) \Delta \mathrm{x} & =0
\end{array}
$$

O conjunto de eqs (4.6) pode ser representado na forma matricial por (4.7).

$$
\left[\begin{array}{c}
\Delta \mathrm{x} \\
\Delta \mu \\
\Delta \lambda
\end{array}\right]=-\left[\begin{array}{lcl}
\nabla_{\mathrm{xx}} \mathrm{L}\left(\mathrm{x}^{*}, \lambda^{*}, \mu^{*}, \varepsilon\right) & \nabla_{\mathrm{x}} \mathrm{h}_{\mathrm{j}}\left(\mathrm{x}^{*}\right) & \nabla_{\mathrm{x}} \mathrm{g}_{\mathrm{i}}\left(\mathrm{x}^{*}\right) \\
\mu_{\mathrm{j}}^{*} \nabla_{\mathrm{x}} \mathrm{h}_{\mathrm{j}}\left(\mathrm{x}^{*}\right) & \mathrm{h}_{\mathrm{j}}\left(\mathrm{x}^{*}\right) & 0 \\
\nabla_{\mathrm{x}} \mathrm{g}_{\mathrm{i}}\left(\mathrm{x}^{*}\right) & 0 & 0
\end{array}\right]^{-1}\left[\begin{array}{l}
0 \\
0 \\
\varepsilon_{\mathrm{i}}
\end{array}\right]
$$

O que resulta de forma compacta no sistema matricial (4.8).

$$
\left[\begin{array}{l}
\mathrm{x}(\varepsilon)-\mathrm{x}^{*} \\
\mu(\varepsilon)-\mu^{*} \\
\lambda(\varepsilon)-\lambda^{*}
\end{array}\right]=-\left[\begin{array}{ccc}
\nabla^{2} \mathrm{~L}^{*} & \mathrm{H}^{*} & \mathrm{G}^{*} \\
\mathrm{M}^{*}\left(\mathrm{H}^{*}\right)^{\mathrm{T}} & \operatorname{diag}\left[\mathrm{h}_{\mathrm{j}}\left(\mathrm{x}^{*}\right)\right] & 0 \\
\left(\mathrm{G}^{*}\right)^{\mathrm{T}} & 0 & 0
\end{array}\right]^{-1}\left[\begin{array}{l}
0 \\
0 \\
\varepsilon
\end{array}\right]
$$

onde:

$$
\nabla^{2} \mathrm{~L}^{*}=\nabla_{\mathrm{xx}}^{2} \mathrm{f}\left(\mathrm{x}^{*}\right)+\nabla_{\mathrm{xx}}^{2} \sum_{\mathrm{i}=1}^{\mathrm{m}} \lambda_{\mathrm{i}} \mathrm{g}_{\mathrm{i}}\left(\mathrm{x}^{*}\right)+\nabla_{\mathrm{xx}}^{2} \sum_{\mathrm{j}=1}^{\mathrm{r}} \mu_{\mathrm{j}} \mathrm{h}_{\mathrm{j}}\left(\mathrm{x}^{*}\right) ; \quad \mathrm{M}^{*}=\left(\begin{array}{ccc}
\mu_{1}^{*} & 0 & 0 \\
0 & \ddots & 0 \\
0 & 0 & \mu_{\mathrm{j}}^{*}
\end{array}\right)
$$




$$
\mathrm{G}^{*}=\left[\nabla \mathrm{g}_{1}\left(\mathrm{x}^{*}\right), \ldots, \nabla \mathrm{g}_{\mathrm{m}}\left(\mathrm{x}^{*}\right)\right] ; \quad \mathrm{H}^{*}=\left[\nabla \mathrm{h}_{1}\left(\mathrm{x}^{*}\right), \ldots, \nabla \mathrm{h}_{\mathrm{r}}\left(\mathrm{x}^{*}\right)\right] ; \quad \varepsilon=\left[\begin{array}{l}
\varepsilon_{1} \\
\vdots \\
\varepsilon_{\mathrm{m}}
\end{array}\right]
$$

Determinamos, dessa forma, a metodologia de sensibilidade que aplicaremos ao problema de FPO, a qual chamaremos de "SFPO".

\subsection{APLICAÇÃO DA METODOLOGIA SFPO}

Para exemplificar, aplicaremos a metodologia SFPO ao problema (4.9). Neste exemplo estimaremos novas soluções depois de realizadas perturbações na restrição de igualdade. Consideremos o seguinte problema:

$$
\begin{array}{ll}
\min & \mathrm{x}_{1}^{2}+\mathrm{x}_{2}^{2} \\
\text { s.a } & \\
& \mathrm{x}_{1}+2 \mathrm{x}_{2}=1+\varepsilon_{1} \\
& \mathrm{x}_{1}^{2}-\mathrm{x}_{2} \geq 0
\end{array}
$$

Para aplicarmos a metodologia SFPO, primeiramente temos que resolver o problema para encontrarmos o ponto ótimo sem perturbação. Após haver sido encontrado o ponto ótimo do problema, utilizaremos a SFPO para estimar um novo ponto quando $\varepsilon_{1} \neq 0$.

A tabela 4.1 apresenta uma legenda dos símbolos utilizados nesta aplicação. 
TABELA 4.1 - Legenda de símbolos

\begin{tabular}{|c|c|}
\hline Símbolo & Significado \\
\hline \hline$*$ & Ponto ótimo sem perturbação utilizado na análise de sensibilidade \\
\hline$\circ$ & Ponto ótimo após perturbação \\
\hline+ & Ponto estimado \\
\hline--- & Curvas de nível da função Objetivo \\
\hline--- & Restrição de desigualdade \\
\hline- & Restrição de igualdade sem perturbação \\
\hline- & Restrição de igualdade com perturbação \\
\hline
\end{tabular}

\subsubsection{OBTENÇÃO DO PONTO ÓTIMO}

Ao problema (4.9), onde todas as restrições estão ativas, podemos associar a seguinte função Lagrangiana:

$\mathrm{L}=\left(\mathrm{x}_{1}^{2}+\mathrm{x}_{2}^{2}\right)+\lambda_{1}\left(\mathrm{x}_{1}+2 \mathrm{x}_{2}-1-\varepsilon_{1}\right)+\mu_{1}\left(\mathrm{x}_{1}^{2}-\mathrm{x}_{2}\right)$

A condição de otimalidade, aplicada à função Lagrangiana resulta no seguinte sistema de equações:

$2 \mathrm{x}_{1}+\lambda_{1}+2 \mu_{1} \mathrm{x}_{1}=0$

$2 \mathrm{x}_{2}+2 \lambda_{1}+\mu_{1}=0$

$\mathrm{x}_{1}+2 \mathrm{x}_{2}-1-\varepsilon_{1}=0$

$\mathrm{x}_{1}^{2}-\mathrm{x}_{2} \quad=0$

Resolvendo o sistema (4.10), obtemos a solução, em função de $\varepsilon_{1}$, pelas expressões a seguir. 


$$
\begin{aligned}
& \mathrm{x}_{1}=\frac{-1+\sqrt{9-8 \varepsilon_{1}}}{4} \\
& \mathrm{x}_{2}=\mathrm{x}_{1}^{2} \\
& \mu_{1}=\frac{2 \mathrm{x}_{2}-4 \mathrm{x}_{1}}{4 \mathrm{x}_{1}+1} \\
& \lambda_{1}=\frac{-2 \mathrm{x}_{2}+\mu_{1}}{2}
\end{aligned}
$$

Para $\varepsilon_{1}=0$, temos o ponto ótimo do problema sem perturbação, representado na tabela 4.2 e na Figura 4.1, que satisfaz KKT.

TABELA 4.2 - Solução ótima do problema, $\varepsilon_{1}=0$

\begin{tabular}{|c|c|c|c|c|c||}
\hline$\varepsilon_{1}=0$ & $\mathrm{x}_{1}$ & $\mathrm{x}_{2}$ & $\lambda_{1}$ & $\mu_{1}$ & $\mathrm{f}(\mathrm{x})$ \\
\hline \hline '* $)$ & 0,5000 & 0,2500 & $-0,5000$ & $-0,5000$ & 0,3125 \\
\hline
\end{tabular}

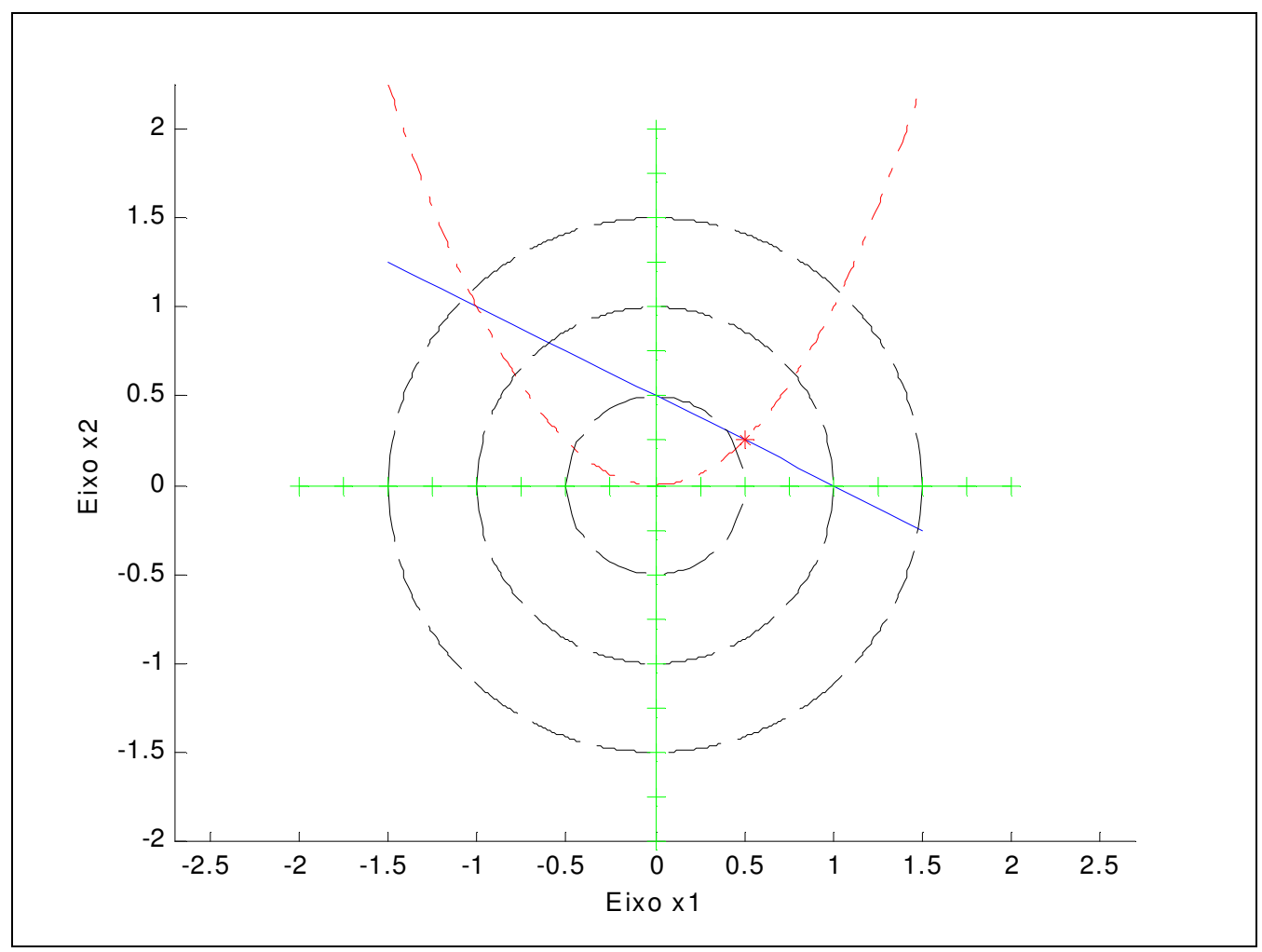

Figura 4.1 - Solução ótima do problema, $\varepsilon_{1}=0$ 


\subsubsection{ESTIMAÇÃO DO NOVO PONTO VIA SFPO}

Obtido o ponto ótimo para o problema (4.9), aplicamos a metodologia SFPO para estima novas soluções ao problema perturbado, isto é, com $\varepsilon_{1} \neq 0$.

Da eq.(4.8) temos o seguinte sistema,

$$
\left[\begin{array}{l}
\mathrm{x}(\varepsilon) \\
\mu(\varepsilon) \\
\lambda(\varepsilon)
\end{array}\right]=\left[\begin{array}{l}
\mathrm{x}^{*} \\
\mu^{*} \\
\lambda^{*}
\end{array}\right]-\left[\begin{array}{cccc}
\nabla^{2} \mathrm{~L}^{*} & \mathrm{H}^{*} & \mathrm{G}^{*} & \\
\mathrm{M}^{*}\left(\mathrm{H}^{*}\right)^{\mathrm{T}} & \operatorname{diag}\left[\mathrm{h}_{\mathrm{j}}\left(\mathrm{x}^{*}\right)\right] & & 0 \\
\left(\mathrm{G}^{*}\right)^{\mathrm{T}} & 0 & 0
\end{array}\right]^{-1}\left[\begin{array}{l}
0 \\
0 \\
\varepsilon_{\mathrm{i}}
\end{array}\right]
$$

que após substituídos os valores da tabela 4.2, resulta no seguinte sistema:

$$
\left[\begin{array}{l}
\mathrm{x}_{1}\left(\varepsilon_{1}\right) \\
\mathrm{x}_{2}\left(\varepsilon_{1}\right) \\
\mu_{1}\left(\varepsilon_{1}\right) \\
\lambda_{1}\left(\varepsilon_{1}\right)
\end{array}\right]=\left[\begin{array}{l}
0,5000 \\
0,2500 \\
-0,500 \\
-0,500
\end{array}\right]-\left[\begin{array}{cccc}
1,0000 & 0,0000 & -1,0000 & 1,0000 \\
0,0000 & 2,0000 & 1,0000 & 2,0000 \\
0,5000 & -0,5000 & 0,0000 & 0,0000 \\
1,0000 & 2,0000 & 0,0000 & 0,0000
\end{array}\right]^{-1}\left[\begin{array}{c}
0,0000 \\
0,0000 \\
0,0000 \\
\varepsilon_{1}
\end{array}\right],
$$

onde as variáveis em função de $\varepsilon_{1}$ são determinadas como segue.

$$
\left[\begin{array}{l}
\mathrm{x}_{1}\left(\varepsilon_{1}\right) \\
\mathrm{x}_{2}\left(\varepsilon_{1}\right) \\
\mu_{1}\left(\varepsilon_{1}\right) \\
\lambda_{1}\left(\varepsilon_{1}\right)
\end{array}\right]=\left[\begin{array}{c}
0,5000 \\
0,2500 \\
-0,500 \\
-0,500
\end{array}\right]-\left[\begin{array}{c}
0,3333 \varepsilon_{1} \\
0,3333 \varepsilon_{1} \\
0,0000 \varepsilon_{1} \\
-0,3333 \varepsilon_{1}
\end{array}\right]
$$




\subsubsection{VALIDAÇÃO DA METODOLOGIA SFPO}

Para o problema (4.9) aplicaremos uma perturbação de $\varepsilon_{1}=0,02, \varepsilon_{1}=0,10 \mathrm{e}$ $\varepsilon_{1}=0,20$, que corresponde à variação de $2 \%, 10 \%$ e $20 \%$ nos recursos da restrição de igualdade. Para cada caso compararemos a solução via SFPO com a solução obtida através das eqs.(4.11).

A tabela 4.3 mostra os pontos obtidos via SFPO, “+”, e via equações (4.11), “o”, para $\varepsilon_{1}=0,02$, os quais podem ser visualizados na Figura 4.2.

TABELA 4.3 - Soluções pós-perturbação, $\varepsilon_{1}=0,02$

\begin{tabular}{|c|c|c|c|c|c||}
\hline \hline$\varepsilon_{1}=0,02$ & $\mathrm{x}_{1}$ & $\mathrm{x}_{2}$ & $\lambda_{1}$ & $\mu_{1}$ & $\mathrm{f}(\mathrm{x})$ \\
\hline \hline 'o' & 0,4933 & 0,2433 & $-0,4933$ & $-0,5000$ & 0,3025 \\
\hline '+' & 0,4933 & 0,2433 & $-0,4933$ & $-0,5000$ & 0,3025 \\
\hline
\end{tabular}

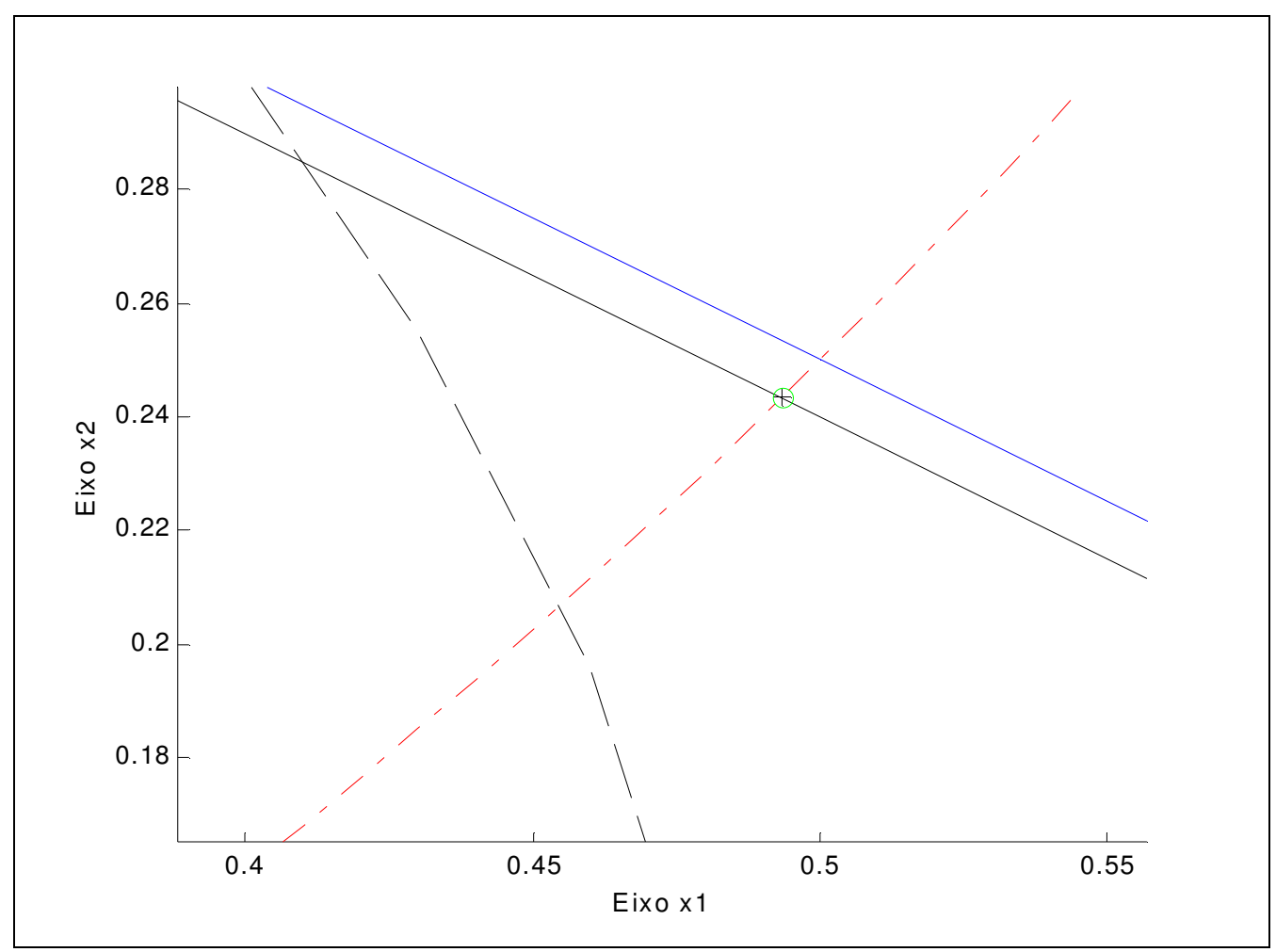

Figura 4.2 - Solução do problema para $\varepsilon_{1}=0,02$ 
A tabela 4.4 mostra os pontos obtidos via SFPO, “+”, e via eqs.(4.11), “o”, para $\varepsilon_{1}=0,10$, os quais podem ser visualizados na Figura 4.3.

TABELA 4.4 - Soluções pós-perturbação, $\varepsilon_{1}=0,10$

\begin{tabular}{||c|c|c|c|c|c||}
\hline \hline$\varepsilon_{1}=0,10$ & $\mathrm{x}_{1}$ & $\mathrm{x}_{2}$ & $\lambda_{1}$ & $\mu_{1}$ & $\mathrm{f}(\mathrm{x})$ \\
\hline \hline 'o' & 0,4659 & 0,2171 & $-0,4666$ & $-0,4992$ & 0,2649 \\
\hline$+'$ & 0,4667 & 0,2167 & $-0,4667$ & 0,5000 & 0,2647 \\
\hline
\end{tabular}

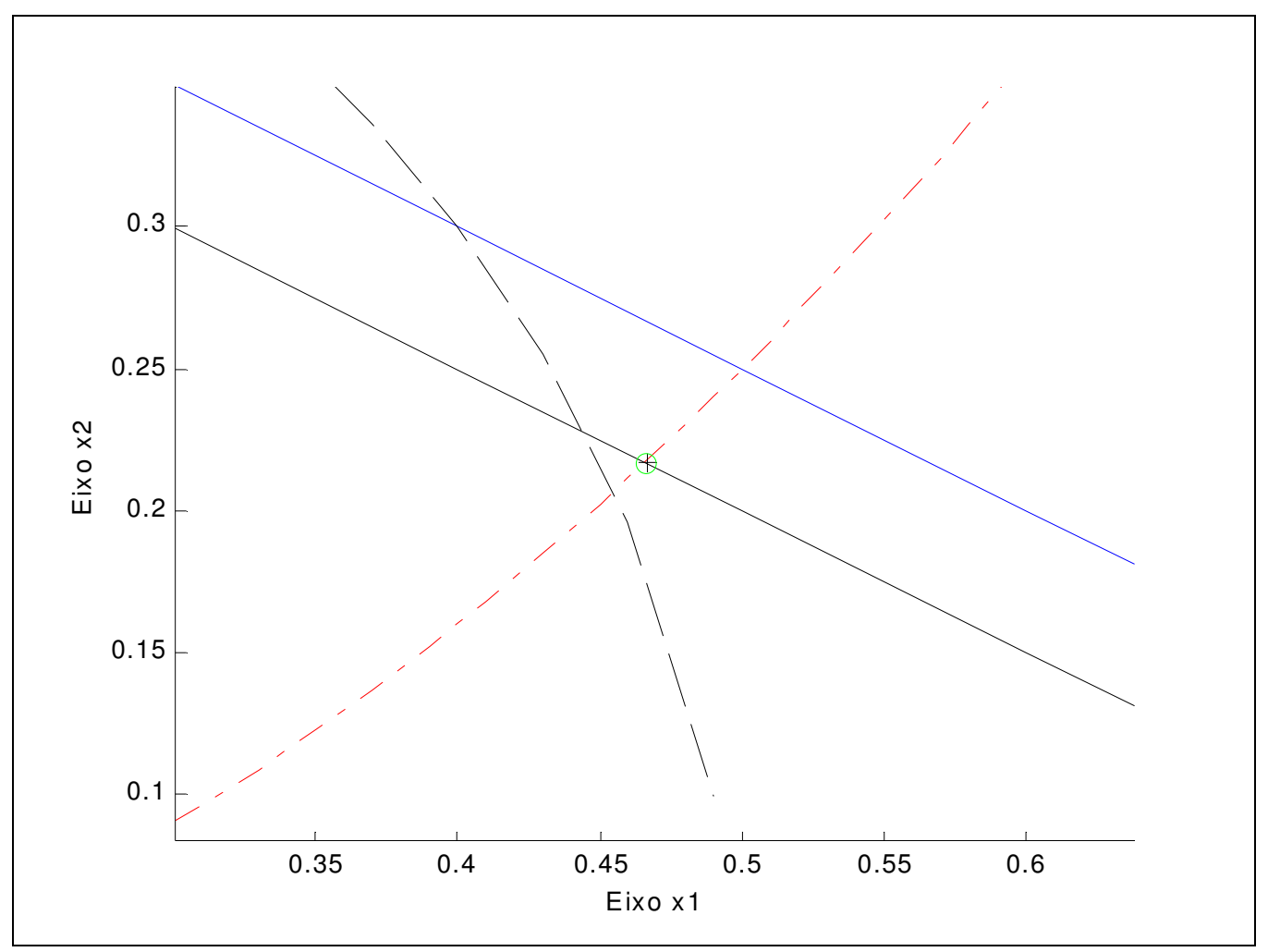

Figura 4.3 - Solução do problema para $\varepsilon_{1}=0,10$

A tabela 4.5 mostra os pontos obtidos via SFPO, “+”, e via as equações (4.11), “o”, para $\varepsilon_{1}=0,20$, os quais podem ser visualizados na Figura 4.4. 
TABELA 4.5 - Soluções pós-perturbação, $\varepsilon_{1}=0,20$

\begin{tabular}{|c|c|c|c|c|c||}
\hline$\varepsilon_{1}=0,20$ & $\mathrm{x}_{1}$ & $\mathrm{x}_{2}$ & $\lambda_{1}$ & $\mu_{1}$ & $\mathrm{f}(\mathrm{x})$ \\
\hline \hline$' \mathrm{O} '$ & 0,4301 & 0,1850 & $-0,4332$ & $-0,4964$ & 0,2192 \\
\hline$'+'$ & 0,4333 & 0,1833 & $-0,4333$ & $-0,5000$ & 0,2213 \\
\hline
\end{tabular}

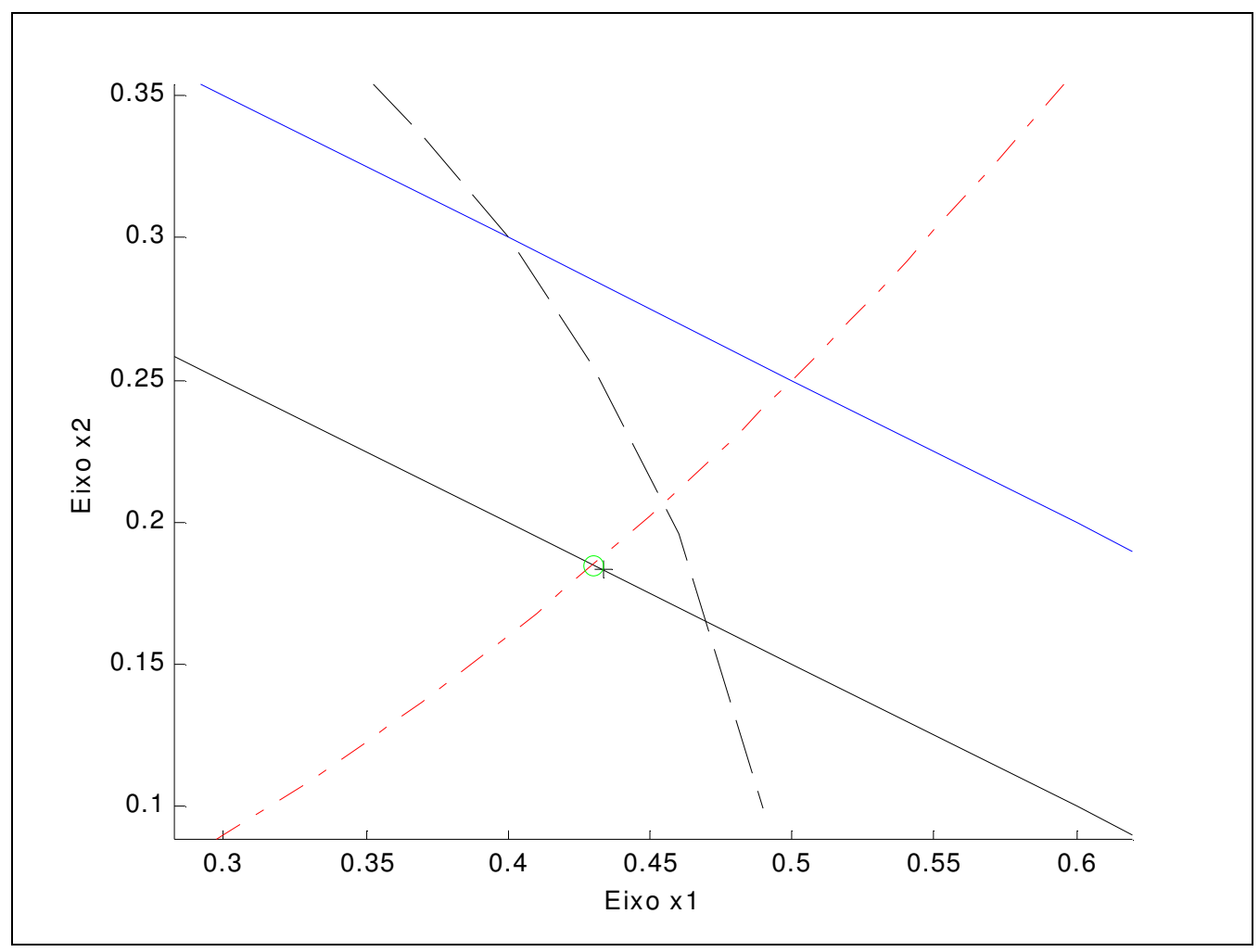

Figura 4.4 - Solução do problema para $\varepsilon_{1}=0,20$

A aplicação mostrou que a metodologia SFPO pode ser aplicada em problemas de PNL com bons resultados. Quanto menor a perturbação, melhor o resultado estimado via SFPO. A obtenção de novas soluções via SFPO, ao contrário dos métodos de resolução de problemas PNL, não é iterativo e não depende da escolha de parâmetros iniciais e de correção. No capítulo 6 mostraremos vários exemplos da metodologia aplicada ao problema de FPO. 


\section{Capítulo 5}

\section{SENSIBILIDADE APLICADA AO PROBLEMA DE FPO}

\subsection{INTRODUÇÃO}

Neste capítulo, aplicaremos a metodologia SFPO ao problema de FPO, mostraremos o algoritmo de resolução e discutiremos sua implementação computacional.

\subsection{APLICAÇÃO DA METODOLOGIA SFPO AO PROBLEMA DE FLUXO DE POTÊNCIA ÓTIMO}

Um sistema opera em regime permanente, porém devido a flutuação nas demandas uma perturbação é introduzida ao sistema. Se introduzirmos esta perturbação ao problema de FPO, resulta o seguinte problema perturbado. 


$$
\begin{array}{lll}
\min & \sum_{\mathrm{m} \in \Omega \mathrm{k}} \mathrm{g}_{\mathrm{km}}\left(\mathrm{V}_{\mathrm{k}}^{2}+\mathrm{V}_{\mathrm{m}}^{2}-2 \mathrm{~V}_{\mathrm{k}} \mathrm{V}_{\mathrm{m}} \cos \theta_{\mathrm{km}}\right) & \\
\text { s.a. } & \mathrm{P}_{\mathrm{k}}-\sum_{\mathrm{m} \in \Omega \mathrm{k}} \mathrm{P}_{\mathrm{km}}+\varepsilon_{\mathrm{k}}^{\mathrm{P}}=0 & \mathrm{k}=1, \cdots, \mathrm{NBCCR} \\
& \mathrm{Q}_{\mathrm{k}}+\mathrm{Q}_{\mathrm{k}}^{\mathrm{sh}}-\sum_{\mathrm{m} \in \Omega \mathrm{k}} \mathrm{Q}_{\mathrm{km}}+\varepsilon_{\mathrm{k}}^{\mathrm{Q}}=0 & \mathrm{k}=1, \cdots, \mathrm{NBC} \\
& \mathrm{Q}_{\mathrm{k}}^{\min }+\mathrm{Q}_{\mathrm{k}}^{\mathrm{sh}} \leq \sum_{\mathrm{m} \in \Omega \mathrm{k}} \mathrm{Q}_{\mathrm{km}} \leq \mathrm{Q}_{\mathrm{k}}^{\max }+\mathrm{Q}_{\mathrm{k}}^{\mathrm{sh}} & \mathrm{k}=1, \cdots, \mathrm{NBCR} \\
& \mathrm{V}_{\mathrm{k}}^{\min } \leq \mathrm{V}_{\mathrm{k}} \leq \mathrm{V}_{\mathrm{k}}^{\max } & \mathrm{k}=1, \cdots, \mathrm{NB}
\end{array}
$$

onde:

$\varepsilon_{\mathrm{k}}^{\mathrm{P}}$ - vetor perturbação relacionado com as equações de igualdade das potências ativas;

$\varepsilon_{\mathrm{k}}^{\mathrm{Q}}$ - vetor perturbação relacionado com as equações de igualdade das potências reativas.

Chamaremos as restrições:

$\mathrm{P}_{\mathrm{K}}-\sum_{\mathrm{m} \in \Omega \mathrm{k}} \mathrm{P}_{\mathrm{km}}+\varepsilon_{\mathrm{k}}^{\mathrm{P}}=0$ e $\mathrm{Q}_{\mathrm{K}}+\mathrm{Q}_{\mathrm{K}}^{\mathrm{sh}}-\sum_{\mathrm{m} \in \Omega \mathrm{k}} \mathrm{Q}_{\mathrm{km}}+\varepsilon_{\mathrm{k}}^{\mathrm{Q}}$, de $\mathrm{g}_{\mathrm{i}}$ onde $\mathrm{i}=1, \ldots, \mathrm{m} ;$

$\mathrm{Q}_{\mathrm{K}}^{\min }+\mathrm{Q}_{\mathrm{K}}^{\mathrm{sh}} \leq \sum_{\mathrm{m} \in \Omega \mathrm{k}} \mathrm{Q}_{\mathrm{km}} \leq \mathrm{Q}_{\mathrm{K}}^{\max }+\mathrm{Q}_{\mathrm{K}}^{\mathrm{sh}} \quad$ e $\quad \mathrm{V}_{\mathrm{K}}^{\min } \leq \mathrm{V}_{\mathrm{K}} \leq \mathrm{V}_{\mathrm{K}}^{\max }$, de $\mathrm{h}_{\mathrm{j}}$ onde $\mathrm{j}=1, \ldots, \mathrm{r} ;$

e a função objetivo,

$$
\sum_{\mathrm{m} \in \Omega \mathrm{k}} \mathrm{g}_{\mathrm{km}}\left(\mathrm{V}_{\mathrm{k}}^{2}+\mathrm{V}_{\mathrm{m}}^{2}-2 \mathrm{~V}_{\mathrm{k}} \mathrm{V}_{\mathrm{m}} \cos \theta_{\mathrm{km}}\right), \text { de } \mathrm{f} .
$$


Utilizamos o FPO para obter a solução para o problema sem perturbação, ou seja, para $\varepsilon=0$. Obtida a solução, associamos ao problema a seguinte função Lagrangina

$$
\mathrm{L}=\mathrm{f}^{*}+\sum_{\mathrm{i}=1}^{\mathrm{m}} \lambda_{\mathrm{i}}^{*} \mathrm{~g}_{\mathrm{i}}^{*}+\sum_{\mathrm{j}=1}^{\mathrm{r}} \mu_{\mathrm{j}}^{*} \mathrm{~h}_{\mathrm{j}}^{*}
$$

onde $\mathrm{f}, \mathrm{g}$ e $\mathrm{h}$ estão em função das variáveis: magnitude de tensão , $\mathrm{V}^{*}$, e ângulo de fase, $\theta^{*}$.

Como apresentado, a fórmula compacta da SFPO é dada por:

$\left[\begin{array}{l}\mathrm{x}(\varepsilon)-\mathrm{x}^{*} \\ \mu(\varepsilon)-\mu^{*} \\ \lambda(\varepsilon)-\lambda^{*}\end{array}\right]=-\left[\begin{array}{ccc}\nabla^{2} \mathrm{~L}^{*} & \mathrm{H}^{*} & \mathrm{G}^{*} \\ \mathrm{M}^{*}\left(\mathrm{H}^{*}\right)^{\mathrm{T}} & \operatorname{diag}\left(\mathrm{h}_{\mathrm{j}}^{*}\right) & 0 \\ \left(\mathrm{G}^{*}\right)^{\mathrm{T}} & 0 & 0\end{array}\right]^{-1}\left[\begin{array}{l}0 \\ 0 \\ \varepsilon\end{array}\right]$.

Com a finalidade de facilitar a representação do sistema, chamaremos a matriz,

$\left[\begin{array}{c:c:c}\nabla^{2} \mathrm{~L}^{*} & \mathrm{H}^{*} & \mathrm{G}^{*} \\ \hdashline \mathrm{M}^{*}\left(\mathrm{H}^{*}\right)^{\mathrm{T}} & \operatorname{diag}\left(\mathrm{h}_{\mathrm{j}}^{*}\right) & 0 \\ \hdashline\left(\mathrm{G}^{*}\right)^{\mathrm{T}} & 0 & 0\end{array}\right]$, de $\mathrm{S}^{*}$, e o vetor, $\left[\begin{array}{l}0 \\ 0 \\ \varepsilon\end{array}\right]$, de $\mathrm{E}$.

As sub-matrizes de $S^{*}$ podem ser representadas por: 
$\nabla^{2} \mathrm{~L}^{*}=\left[\begin{array}{cccc:ccc}\nabla_{\theta_{1} \theta_{1}}^{2} \mathrm{~L}^{*} & \cdots & \nabla_{\theta_{1} \theta_{\mathrm{N}}}^{2} \mathrm{~L}^{*} & \nabla_{\theta_{1} \mathrm{~V}_{1}}^{2} \mathrm{~L}^{*} & \cdots & \nabla_{\theta_{1} \mathrm{~V}_{\mathrm{N}}}^{2} \mathrm{~L}^{*} \\ \vdots & \ddots & \vdots & \vdots & \ddots & \vdots \\ \nabla_{\theta_{\mathrm{N}} \theta_{1}}^{2} \mathrm{~L}^{*} & \cdots & \nabla_{\theta_{\mathrm{N}} \theta_{\mathrm{N}}}^{2} \mathrm{~L}^{*} & \nabla_{\theta_{\mathrm{N}} \mathrm{V}_{1}}^{2} \mathrm{~L}^{*} & \cdots & \nabla_{\theta_{\mathrm{N}} \mathrm{V}_{\mathrm{N}}}^{2} \mathrm{~L}^{*} \\ \nabla_{\mathrm{V}_{1} \theta_{1}}^{2} \mathrm{~L}^{*} & \cdots & \nabla_{\mathrm{V}_{1} \theta_{\mathrm{N}}}^{2} \mathrm{~L}^{*} & \nabla_{\mathrm{v}_{1} \mathrm{~V}_{1}}^{2} \mathrm{~L}^{*} & \cdots & \nabla_{\mathrm{V}_{1} \mathrm{~V}_{\mathrm{N}}}^{2} \mathrm{~L}^{*} \\ \vdots & \ddots & \vdots & \vdots & \ddots & \vdots \\ \nabla_{\mathrm{V}_{\mathrm{N}} \theta_{1}}^{2} \mathrm{~L}^{*} & \cdots & \nabla_{\mathrm{V}_{\mathrm{N}} \theta_{\mathrm{N}}}^{2} \mathrm{~L}^{*} & \nabla_{\mathrm{V}_{\mathrm{N}} \mathrm{V}_{1}}^{2} \mathrm{~L}^{*} & \cdots & \nabla_{\mathrm{V}_{\mathrm{N}} \mathrm{V}_{\mathrm{N}}}^{2} \mathrm{~L}^{*}\end{array}\right] ; \quad \mathrm{H}^{*}=\left[\begin{array}{cccc}\nabla_{\theta_{1}} \mathrm{~h}_{1}^{*} & \ldots & \nabla_{\theta_{1}} \mathrm{~h}_{\mathrm{r}}^{*} \\ \vdots & \ddots & \vdots \\ \nabla_{\theta_{\mathrm{N}}} \mathrm{h}_{1}^{*} & \cdots & \nabla_{\theta_{\mathrm{N}}} \mathrm{h}_{\mathrm{r}}^{*} \\ \hdashline \nabla_{\mathrm{V}_{1}} \mathrm{~h}_{1}^{*} & \ldots & \nabla_{\mathrm{V}_{1}} \mathrm{~h}_{\mathrm{r}}^{*} \\ \vdots & \ddots & \vdots \\ \nabla_{\mathrm{V}_{\mathrm{N}}} \mathrm{h}_{1}^{*} & \cdots & \nabla_{\mathrm{V}_{\mathrm{N}}} \mathrm{h}_{\mathrm{r}}^{*}\end{array}\right]$

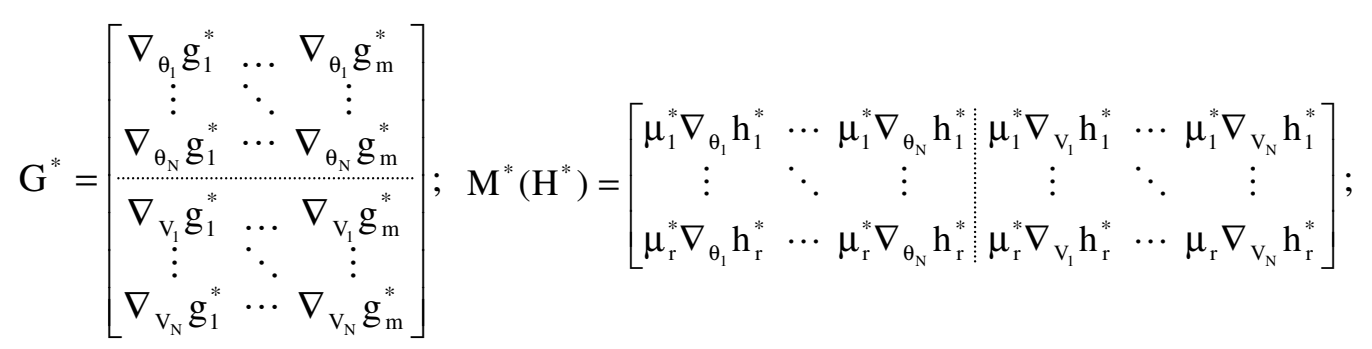

$\left(\mathrm{G}^{*}\right)^{\mathrm{T}}=\left[\begin{array}{cccc:ccc}\nabla_{\theta_{1}} \mathrm{~g}_{1}^{*} & \cdots & \nabla_{\theta_{\mathrm{N}}} \mathrm{g}_{1}^{*} & \nabla_{\mathrm{V}_{1}} \mathrm{~g}_{1}^{*} & \ldots & \nabla_{\mathrm{V}_{\mathrm{N}}} \mathrm{g}_{1}^{*} \\ \vdots & \ddots & \vdots & \vdots & \ddots & \vdots \\ \nabla_{\theta_{1}} \mathrm{~g}_{\mathrm{m}}^{*} & \cdots & \nabla_{\theta_{\mathrm{N}}} \mathrm{g}_{\mathrm{m}}^{*} & \nabla_{\mathrm{V}_{1}} \mathrm{~g}_{\mathrm{m}}^{*} & \cdots & \nabla_{\mathrm{V}_{\mathrm{N}}} \mathrm{g}_{\mathrm{m}}^{*}\end{array}\right] ; \quad \operatorname{diag}\left(\mathrm{h}_{\mathrm{j}}^{*}\right)=\left[\begin{array}{ccc}\mathrm{h}_{1}^{*} & \cdots & 0 \\ \vdots & \ddots & \vdots \\ 0 & \cdots & \mathrm{h}_{\mathrm{r}}^{*}\end{array}\right]$.

E o vetor $\varepsilon$, de E, por:

$\varepsilon=\left(\begin{array}{c}\varepsilon_{1}^{\mathrm{P}} \\ \vdots \\ \varepsilon_{\mathrm{NBCCR}}^{\mathrm{P}} \\ \varepsilon_{1}^{\mathrm{Q}} \\ \vdots \\ \varepsilon_{\mathrm{NBCR}}^{\mathrm{Q}}\end{array}\right)$

A seguinte expressão é usada para estimar novas soluções quando alguma perturbação ocorrer no problema. O sucesso da abordagem está na obtenção das variáveis ótimas do problema, utilizadas na construção da matriz $\mathrm{S}^{*}$. Por tanto, é 
necessária a utilização de um método de FPO que forneça, além das variáveis ótimas primais, as variáveis ótimas duais.

$$
\left[\begin{array}{l}
\mathrm{x}(\varepsilon) \\
\mu(\varepsilon) \\
\lambda(\varepsilon)
\end{array}\right]=\left[\begin{array}{l}
\mathrm{x}^{*} \\
\mu^{*} \\
\lambda^{*}
\end{array}\right]-\left[\mathrm{S}^{*}\right]^{-1}[\mathrm{E}]
$$

\subsection{RESOLUÇÃO DO PROBLEMA DE FLUXO DE POTÊNCIA ÓTIMO PERTURBADO VIA SFPO}

A metodologia SFPO pode ser representada pelos seguintes passos:

I1) Determine o vetor solução $\left(\mathrm{x}^{*}, \lambda^{*}, \mu^{*}\right)$ para o problema de FPO;

I2) Determine a matriz $\mathrm{S}^{*}$;

I3) Determine o vetor perturbação E;

I4) Resolva o sistema (5.3);

I5) FIM.

O maior esforço computacional do algoritmo SFPO está na resolução do sistema linear (5.3). A seguir apresentaremos a técnica de esparsidade utilizada na resolução do sistema.

\subsection{TÉCNICA DE ESPARSIDADE UTILIZADA}

Considerando as características da matriz gerada na solução da SFPO, simétrica de posições e não de valores, optamos por utilizar a sub-rotina MA28, do 
pacote de sub-rotinas desenvolvidas por L.S Duff e J.K. Reid, do Rutherford Appleton Laboratory. A seguir faremos uma pequena apresentação da sub-rotina.

A sub-rotina MA28 foi apresentada por DUFF \& REID (1979), constitui em um método direto de resolução de sistemas do tipo $A x=b$. O método é uma variante da eliminação Gauss para sistemas esparsos. Dada uma matriz esparsa A, a sub-rotina MA28 pode ser usada para se obter a decomposição LU de uma permutação de A:

$\mathrm{PAQ}=\mathrm{LU}$

onde $\mathrm{P}$ e Q são matrizes de permutação, L é uma matriz triangular inferior unitária e U é a matriz triangular superior.

O método é dividido em três estágios. No primeiro a matriz A é decomposta em fatores, através de uma estratégia designada a manter a esparsidade, minimizar o número de fill-ins, através da escolha dos pivô. O esquema para seleção do pivô é baseado em MARKOWITZ (1957) que seleciona um elemento diferente de zero para pivô que produza um menor número de fill-ins. Para evitar que o pivô escolhido seja de valor pequeno, o que causaria instabilidade numérica, realiza-se um teste.

$$
\left|a_{\mathrm{kk}}^{(\mathrm{k})}\right| \geq \mathrm{u} \cdot \max _{\mathrm{i}}\left|\mathrm{a}_{\mathrm{ki}}^{(\mathrm{k})}\right|
$$


onde u é um parâmetro de entrada e tem que satisfazer ao intervalo $0 \leq \mathrm{u} \leq 1$, onde valores entre 0,1 e 0,25 são usualmente satisfatórios na prática. No segundo estágio, a matriz é fatorada seguindo a ordem de pivoteamento, definida anteriormente no primeiro estágio. A estratégia de fatoração também faz com que possa haver melhor controle quando a matriz A é singular, ou possua elementos nulos na diagonal. No último estágio, é obtida a solução do sistema, o vetor de entrada b é utilizado como vetor solução x. Caso o vetor b seja esparso, essa esparsidade também é explorada.

\subsection{IMPLEMENTAÇÃO COMPUTACIONAL}

A implementação computacional do programa de SFPO foi realizada em linguagem FORTRAN. O programa foi desenvolvido em um micro-computador Pentium III - $800 \mathrm{MHz}$, com 256 Mbytes de memória RAM, do Laboratório de Otimização de Sistemas Elétricos de Potência (LOSEP), do Departamento de Engenharia Elétrica da Escola de Engenharia de São Carlos (EESC - USP). Na implementação utilizamos a dupla precisão aritmética.

O programa foi dividido nos seguintes módulos, mostrados na Figura 5.1: 


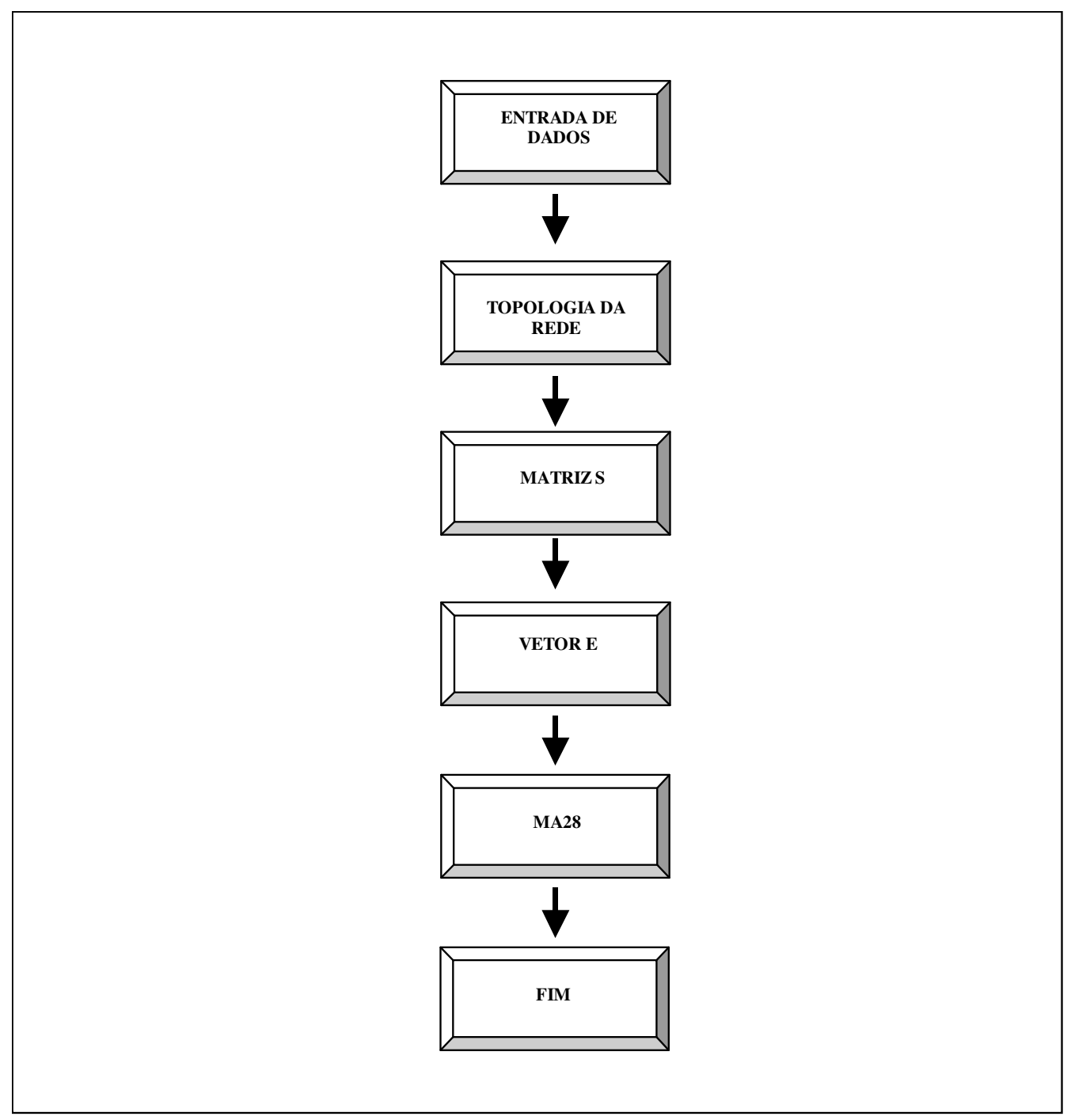

Figura 5.1 - Módulos do programa da metodologia SFPO

Onde:

ENTRADA DE DADOS - faz a leitura de dois bancos de dados. Primeiramente, a leitura do banco de dados do sistema elétrico, e, em seguida, a leitura do banco de dados das variáveis ótimas do sistema, obtidas via FPO. 
TOPOLOGIA DA REDE - realiza a construção dos vetores que contêm as informações da configuração do sistema, ou seja, as ligações físicas existentes entre as barras do sistema elétrico;

MATRIZ S - identifica as posições da matriz $\mathrm{S}$ e numera essas posições. A matriz $\mathrm{S}$ é uma matriz esparsa. Tal esparsidade é explorada de forma a otimizar o desempenho computacional;

VETOR E - constrói o vetor E com as possíveis perturbações do sistema.

MA28 - resolve o sistema (5.3) com o auxílio da sub-rotina MA28;

FIM - o programa é finalizado e o arquivo de saída pode ser lido pelo usuário.

O programa não é iterativo e não depende de parâmetros de entrada. O maior esforço computacional está na resolução do sistema linear (5.3), em que foi utilizada a sub-rotina MA28. 


\section{RESULTADOS NUMÉRICOS}

\subsection{INTRODUÇÃO}

Neste capítulo apresentaremos os resultados numéricos obtidos utilizando a metodologia SFPO. Analisaremos os sistemas elétricos: 3 barras, 9 barras, IEEE 14, IEEE 30, CESP 53, IEEE 118, IEEE 162 e o IEEE 300.

No sistema de 3 barras, faremos uma aplicação detalhada para mostrar os passos da metodologia SFPO. Para o sistema IEEE 14 barras, faremos comparações numéricas entre as soluções obtidas pela SFPO e pelo FPO. Para os sistemas, IEEE 30, CESP 53, IEEE 118 e IEEE 162, aplicaremos a metodologia SFPO para diferentes situações e analisaremos os resultados. No sistema IEEE 300, faremos perturbações em um conjunto de barras de carga, analisaremos os resultados e faremos uma análise de sensibilidade deste aumento de carga em relação à injeção de potência reativa nas barras de geração. Para estes testes, utilizaremos o FPO implementado neste trabalho, para encontrar o ponto ótimo para a condição inicial dos sistemas. Nestes testes, a SFPO será aplicada ao problema de despacho ótimo de 
potência reativa. Este é um caso crítico, porque somente a barra slack absorve as variações na demanda.

No sistema de 9 barras, testaremos a metodologia SFPO aplicada ao problema de Despacho Ótimo de Potência Ativa (DOPA). Neste caso todas as barras de geração, inclusive a barra slack, absorverão as perturbações na demanda do sistema.

Em todos os testes apresentados, a função objetivo do problema representa as perdas ativas na transmissão.

Os bancos de dados dos sistemas utilizados nos testes e as Tabelas de convergência do FPO estão no Apêndice A. As Tabelas de resultados dos sistemas IEEE 118, IEEE 162 e IEEE 300 estão apresentadas no Apêndice B, por serem muito extensas. Nessas Tabelas temos o ponto ótimo sem perturbação e o ponto estimado após a perturbação.

\subsection{EXEMPLO 1: SISTEMA 3 BARRAS}

Este problema foi proposto por DOMMEL \& TINNEY (1968). A Figura 6.1 representa o sistema, que apresenta as seguintes características:

- 1 barra de geração (slack) - barra 1 ;

- 1 barra de controle reativo (PV) - barra 2;

- 1 barra de carga (PQ) - barra 3;

- 2 linhas de transmissão. 


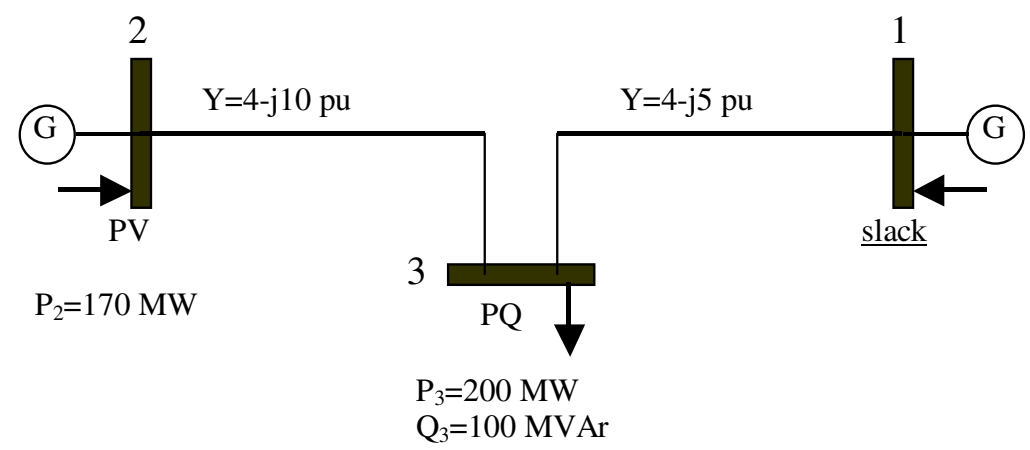

Figura 6.1 - Sistema 3 Barras

As condições iniciais do sistema estão mostradas na Tabela 6.1, e os limites das variáveis, na Tabela 6.2. A solução ótima para o sistema está detalhada nas Tabelas 6.3 e 6.4 .

TABELA 6.1 - Condição inicial do sistema 3 barras

\begin{tabular}{|c||c||c|c||c||c||}
\hline Barra k & Tipo & $\mathrm{V}_{\mathrm{k}}$ (p.u.) & $\theta_{\mathrm{k}}$ (graus) & $\mathrm{P}_{\mathrm{k}}(\mathrm{MW})$ & $\mathrm{Q}_{\mathrm{k}}(\mathrm{MVAr})$ \\
\hline 1 & Slack & 1,0 & 0,0 & --- & --- \\
\hline 2 & PV & 1,0 & 0,0 & 170,0 & --- \\
\hline 3 & PQ & 1,0 & 0,0 & $-200,0$ & $-100,0$ \\
\hline
\end{tabular}

TABELA 6.2 - Limite das variáveis do sistema 3 barras

\begin{tabular}{|c||c||c|c|c||}
\hline Barra $\mathrm{k}$ & $\mathrm{V}_{\mathrm{k}}^{\min }$ (p.u.) & $\mathrm{V}_{\mathrm{k}}^{\max }$ (p.u.) & $\mathrm{Q}_{\mathrm{k}}^{\min }(\mathrm{MVAr})$ & $\mathrm{Q}_{\mathrm{k}}^{\max }(\mathrm{MVAr})$ \\
\hline \hline 1 & 0,8 & 1,2 & -9999 & 9999 \\
\hline 2 & 0,8 & 1,2 & 100 & 200 \\
\hline 3 & 0,9 & 1,0 & --- & --- \\
\hline
\end{tabular}

TABELA 6.3 - Variáveis primais, geração de potência reativa e perdas na transmissão obtidas via FPO para $(\varepsilon=0,0)$

\begin{tabular}{|c||c||c|c||}
\hline \hline Barra $\mathrm{k}$ & $\mathrm{V}_{\mathrm{k}}$ (p.u.) & $\theta_{\mathrm{k}}$ (graus) & $\mathrm{Q}$ (MVAr) \\
\hline \hline 1 & 1,0712 & 0 & 28,4 \\
\hline 2 & 1,1247 & 4,38 & 101,1 \\
\hline 3 & 1,0000 & $-1,32$ & --- \\
\hline \hline \multicolumn{3}{|c|}{ Perdas (MW) } & \multicolumn{3}{|c|}{$12,916 \mathrm{MW}$} \\
\hline
\end{tabular}


TABELA 6.4 - Variáveis duais obtidas via FPO

\begin{tabular}{|c|c|c|c||c||c|c||}
\hline Barra k & $\begin{array}{c}\mu \text { (limite } \\
\text { superior } \\
\text { de } \mathrm{V}_{\mathrm{k}} \text { ) }\end{array}$ & $\begin{array}{c}\mu \text { (limite } \\
\text { inferior de } \\
\mathrm{V}_{\mathrm{k}} \text { ) }\end{array}$ & $\begin{array}{c}\mu \text { (limite } \\
\text { superior } \\
\text { de } \mathrm{Q}_{\mathrm{k}}\end{array}$ & $\begin{array}{c}\mu \text { (limite } \\
\text { inferior } \\
\text { de } \mathrm{Q}_{\mathrm{k}}\end{array}$ & $\begin{array}{c}\lambda \text { (balanço } \\
\text { da potência } \\
\text { ativa) }\end{array}$ & $\begin{array}{c}\lambda \text { (balanço } \\
\text { da potência } \\
\text { reativa) }\end{array}$ \\
\hline \hline 1 & 0,0000 & 0,0000 & --- & --- & --- & --- \\
\hline 2 & 0,0000 & 0,0000 & 0,0000 & 0,0000 & 0,0275 & --- \\
\hline 3 & 0,2524 & 0,0000 & --- & --- & $-0,0793$ & $-0,0499$ \\
\hline
\end{tabular}

Tendo a solução ótima para o caso inicial, podemos estimar novas soluções depois de realizadas perturbações no sistema através da SFPO. O problema perturbado é representado por (6.1).

$$
\begin{array}{ll}
\min & 4\left[\left(\mathrm{~V}_{1}^{2}+\mathrm{V}_{3}^{2}-2 \mathrm{~V}_{1} \mathrm{~V}_{3} \cos \theta_{13}\right)+\left(\mathrm{V}_{2}^{2}+\mathrm{V}_{3}^{2}-2 \mathrm{~V}_{2} \mathrm{~V}_{3} \cos \theta_{23}\right)\right] \\
\text { s.a: } & 1,7-\mathrm{P}_{2}+\varepsilon_{2}^{\mathrm{P}}=0 \\
& -2,0-\mathrm{P}_{3}+\varepsilon_{3}^{\mathrm{P}}=0 \\
& -1,0-\mathrm{Q}_{3}+\varepsilon_{3}^{\mathrm{Q}}=0 \\
& 1,0 \leq \mathrm{Q}_{2} \leq 2,0 \\
& 0,8 \leq \mathrm{V}_{1} \leq 1,2 \\
& 0,8 \leq \mathrm{V}_{2} \leq 1,2 \\
& 0,9 \leq \mathrm{V}_{3} \leq 1,0
\end{array}
$$

em que:

$$
\begin{aligned}
& \mathrm{P}_{2}=4 \mathrm{~V}_{2}^{2}-4 \mathrm{~V}_{2} \mathrm{~V}_{3} \cos \theta_{23}+10 \mathrm{~V}_{2} \mathrm{~V}_{3} \operatorname{sen} \theta_{23} \\
& \mathrm{P}_{3}=\left(4 \mathrm{~V}_{3}^{2}-4 \mathrm{~V}_{3} \mathrm{~V}_{2} \cos \theta_{32}+10 \mathrm{~V}_{3} \mathrm{~V}_{2} \operatorname{sen} \theta_{32}\right)+\left(4 \mathrm{~V}_{3}^{2}-4 \mathrm{~V}_{3} \mathrm{~V}_{1} \cos \theta_{31}+4 \mathrm{~V}_{3} \mathrm{~V}_{1} \operatorname{sen} \theta_{31}\right) ; \\
& \mathrm{Q}_{3}=\left(10 \mathrm{~V}_{3}^{2}-10 \mathrm{~V}_{3} \mathrm{~V}_{2} \cos \theta_{32}-4 \mathrm{~V}_{3} \mathrm{~V}_{2} \operatorname{sen} \theta_{32}\right)+\left(5 \mathrm{~V}_{3}^{2}-5 \mathrm{~V}_{3} \mathrm{~V}_{1} \cos \theta_{31}-4 \mathrm{~V}_{3} \mathrm{~V}_{1} \operatorname{sen} \theta_{31}\right) ; \\
& \mathrm{Q}_{2}=10 \mathrm{~V}_{2}^{2}-10 \mathrm{~V}_{2} \mathrm{~V}_{3} \cos \theta_{23}-4 \mathrm{~V}_{2} \mathrm{~V}_{3} \operatorname{sen} \theta_{23} ; \\
& \cos \theta_{\mathrm{ij}}=\cos \left(\theta_{\mathrm{i}}-\theta_{\mathrm{j}}\right) ; \\
& \operatorname{sen} \theta_{\mathrm{ij}}=\operatorname{sen}\left(\theta_{\mathrm{i}}-\theta_{\mathrm{j}}\right)
\end{aligned}
$$


Associamos à função objetivo e às restrições do problema (6.1), funções auxiliares com a finalidade de simplificar a apresentação da formulação, como apresentado a seguir.

$$
\begin{aligned}
& \min 4\left[\left(\mathrm{~V}_{1}^{2}+\mathrm{V}_{3}^{2}-2 \mathrm{~V}_{1} \mathrm{~V}_{3} \cos \theta_{13}\right)+\left(\mathrm{V}_{2}^{2}+\mathrm{V}_{3}^{2}-2 \mathrm{~V}_{2} \mathrm{~V}_{3} \cos \theta_{23}\right)\right] \rightarrow \mathrm{f}(\mathrm{x}) \\
& \text { s.a : } 1,7-\mathrm{P}_{2}+\varepsilon_{2}^{\mathrm{P}}=0 \quad \rightarrow \mathrm{g}_{1}(\mathrm{x})=0 \\
& -2,0-\mathrm{P}_{3}+\varepsilon_{3}^{\mathrm{P}}=0 \quad \rightarrow \mathrm{g}_{2}(\mathrm{x})=0 \\
& -1,0-\mathrm{Q}_{3}+\varepsilon_{3}^{\mathrm{Q}}=0 \quad \rightarrow \mathrm{g}_{3}(\mathrm{x})=0 \\
& 0,8-\mathrm{V}_{1} \leq 0 \quad \rightarrow \mathrm{h}_{1}(\mathrm{x}) \leq 0 \\
& \mathrm{~V}_{1}-1,2 \leq 0 \\
& 0,8-\mathrm{V}_{2} \leq 0 \\
& \mathrm{~V}_{2}-1,2 \leq 0 \\
& 0,9-\mathrm{V}_{3} \leq 0 \\
& \mathrm{~V}_{3}-1,0 \leq 0 \\
& 1,0-\mathrm{Q}_{2} \leq 0 \\
& \rightarrow \mathrm{h}_{2}(\mathrm{x}) \leq 0 \text { (6.2) } \\
& \mathrm{Q}_{2}-2,0 \leq 0 \\
& \rightarrow \mathrm{h}_{3}(\mathrm{x}) \leq 0 \\
& \rightarrow \mathrm{h}_{4}(\mathrm{x}) \leq 0 \\
& \rightarrow \mathrm{h}_{5}(\mathrm{x}) \leq 0 \\
& \rightarrow \mathrm{h}_{6}(\mathrm{x}) \leq 0 \\
& \rightarrow \mathrm{h}_{7}(\mathrm{x}) \leq 0 \\
& \rightarrow \mathrm{h}_{8}(\mathrm{x}) \leq 0
\end{aligned}
$$

A partir da solução ótima do problema obtida via FPO, iniciamos a aplicação da metodologia SFPO como segue:

A função Lagrangiana associada ao problema perturbado é representada por:

$$
\begin{array}{r}
\mathrm{L}(\mathrm{x}, \lambda, \mu)=\mathrm{f}(\mathrm{x})+\mu_{1} \mathrm{~h}_{1}(\mathrm{x})+\mu_{2} \mathrm{~h}_{2}(\mathrm{x})+\mu_{3} \mathrm{~h}_{3}(\mathrm{x})+\mu_{4} \mathrm{~h}_{4}(\mathrm{x})+\mu_{5} \mathrm{~h}_{5}(\mathrm{x})+ \\
\mu_{6} \mathrm{~h}_{6}(\mathrm{x})+\mu_{7} \mathrm{~h}_{7}(\mathrm{x})+\mu_{8} \mathrm{~h}_{8}(\mathrm{x})+\lambda_{1} \mathrm{~g}_{1}(\mathrm{x})+\lambda_{2} \mathrm{~g}_{2}(\mathrm{x})+\lambda_{3} \mathrm{~g}_{3}(\mathrm{x})
\end{array}
$$

Tendo a função Lagrangiana do problema perturbado, aplicamos a metodologia SFPO, que considera o gradiente da função Lagrangiana, a folga complementar e as restrições de igualdade perturbadas como apresentadas em (6.3). 
$\nabla \mathrm{L}(\mathrm{x}, \lambda, \mu)=0$

$\mu_{1} \mathrm{~h}_{1}=0$

$\mu_{2} h_{2}=0$

$\mu_{3} h_{3}=0$

$\mu_{4} \mathrm{~h}_{4}=0$

$\mu_{5} \mathrm{~h}_{5}=0$

$\mu_{6} \mathrm{~h}_{6}=0$

$\mu_{7} h_{7}=0$

$\mu_{8} \mathrm{~h}_{8}=0$

$\mathrm{g}_{1}(\mathrm{x})=0$

$\mathrm{g}_{2}(\mathrm{x})=0$

$\mathrm{g}_{3}(\mathrm{x})=0$

O sistema de equações não lineares (6.3) é linearizado no ponto $\left(x^{*}, \lambda^{*}, \mu^{*}\right)$, o que resulta no seguinte sistema linear:

$\left[\begin{array}{l}\mathrm{x}(\varepsilon) \\ \gamma(\varepsilon) \\ \lambda(\varepsilon)\end{array}\right]=\left[\begin{array}{c}\mathrm{x}^{*} \\ \gamma^{*} \\ \lambda^{*}\end{array}\right]-\left[\begin{array}{c:c:c}\nabla^{2} \mathrm{~L}^{*} & \mathrm{H}^{*} & \mathrm{G}^{*} \\ \hdashline \mathrm{M}^{*}\left(\mathrm{H}^{*}\right)^{\mathrm{T}} & \operatorname{diag}\left(\mathrm{h}_{\mathrm{j}}^{*}\right) & 0 \\ \hdashline\left(\mathrm{G}^{*}\right)^{\mathrm{T}} & 0 & 0\end{array}\right]^{-1}\left[\begin{array}{l}0 \\ 0 \\ \varepsilon\end{array}\right]$.

Onde as sub-matrizes de $\mathrm{S}^{*}$ do problema são determinadas por: 


$$
\begin{aligned}
& {\left[\frac{\partial^{2} L^{*}}{\partial_{\mathrm{v} 1} \partial_{\mathrm{v} 1}} \quad 0 \quad \frac{\partial^{2} \mathrm{~L}^{*}}{\partial_{\mathrm{v} 1} \partial_{\mathrm{v} 3}}: 0 \frac{\partial^{2} \mathrm{~L}^{*}}{\partial_{\mathrm{v} 1} \partial_{\theta 3}}\right.} \\
& 0 \quad \frac{\partial^{2} L^{*}}{\partial_{\mathrm{v} 2} \partial_{\mathrm{v} 2}} \frac{\partial^{2} \mathrm{~L}^{*}}{\partial_{\mathrm{v} 2} \partial_{\mathrm{v} 3}} \frac{\partial^{2} \mathrm{~L}^{*}}{\partial_{\mathrm{v} 2} \partial_{\theta 2}} \frac{\partial^{2} \mathrm{~L}^{*}}{\partial_{\mathrm{v} 2} \partial_{\theta 3}} \\
& \nabla^{2} L^{*}=\frac{\partial^{2} L^{*}}{\partial_{v 3} \partial_{v 1}} \frac{\partial^{2} L^{*}}{\partial_{v 3} \partial_{v 2}} \frac{\partial^{2} L^{*}}{\partial_{v 3} \partial_{v 3}} \frac{\partial^{2} L^{*}}{\partial_{v 3} \partial_{\theta 2}} \frac{\partial^{2} L^{*}}{\partial_{v 3} \partial_{\theta 3}} \\
& 0 \quad \frac{\partial^{2} L^{*}}{\partial_{\theta 2} \partial_{v 2}} \frac{\partial^{2} L^{*}}{\partial_{\theta 2} \partial_{v 3}} \frac{\partial^{2} L^{*}}{\partial_{\theta 2} \partial_{\theta 2}} \frac{\partial^{2} L^{*}}{\partial_{\theta 2} \partial_{\theta 3}} \\
& {\left[\frac{\partial^{2} L^{*}}{\partial_{\theta 3} \partial_{v 1}} \frac{\partial^{2} L^{*}}{\partial_{\theta 3} \partial_{v 2}} \frac{\partial^{2} L^{*}}{\partial_{\theta 3} \partial_{v 3}} \frac{\partial^{2} L^{*}}{\partial_{\theta 3} \partial_{\theta 2}} \frac{\partial^{2} L^{*}}{\partial_{\theta 3} \partial_{\theta 3}}\right]}
\end{aligned}
$$

$\mathrm{H}=\left[\begin{array}{cccccccc}\frac{\partial \mathrm{h}_{1}^{*}}{\partial \mathrm{V}_{1}} & \frac{\partial \mathrm{h}_{2}^{*}}{\partial \mathrm{V}_{1}} & 0 & 0 & 0 & 0 & 0 & 0 \\ 0 & 0 & \frac{\partial \mathrm{h}_{3}^{*}}{\partial \mathrm{V}_{2}} & \frac{\partial \mathrm{h}_{4}^{*}}{\partial \mathrm{V}_{2}} & 0 & 0 & \frac{\partial \mathrm{h}_{7}^{*}}{\partial \mathrm{V}_{2}} & \frac{\partial \mathrm{h}_{8}^{*}}{\partial \mathrm{V}_{2}} \\ 0 & 0 & 0 & 0 & \frac{\partial \mathrm{h}_{5}^{*}}{\partial \mathrm{V}_{3}} & \frac{\partial \mathrm{h}_{6}^{*}}{\partial \mathrm{V}_{3}} & \frac{\partial \mathrm{h}_{7}^{*}}{\partial \mathrm{V}_{3}} & \frac{\partial \mathrm{h}_{8}^{*}}{\partial \mathrm{V}_{3}} \\ \hdashline 0 & 0 & 0 & 0 & 0 & 0 & \frac{\partial \mathrm{h}_{7}^{*}}{\partial \theta_{2}} & \frac{\partial \mathrm{h}_{8}^{*}}{\partial \theta_{2}} \\ 0 & 0 & 0 & 0 & 0 & 0 & \frac{\partial \mathrm{h}_{7}^{*}}{\partial \theta_{3}} & \frac{\partial \mathrm{h}_{8}^{*}}{\partial \theta_{3}}\end{array}\right]$

$$
\mathrm{G}=\left[\begin{array}{ccc}
0 & \frac{\partial \mathrm{g}_{2}^{*}}{\partial \mathrm{V}_{1}} & \frac{\partial \mathrm{g}_{3}^{*}}{\partial \mathrm{V}_{1}} \\
\frac{\partial \mathrm{g}_{1}^{*}}{\partial \mathrm{V}_{2}} & \frac{\partial \mathrm{g}_{2}^{*}}{\partial \mathrm{V}_{2}} & \frac{\partial \mathrm{g}_{3}^{*}}{\partial \mathrm{V}_{2}} \\
\frac{\partial \mathrm{g}_{1}^{*}}{\partial \mathrm{V}_{3}} & \frac{\partial \mathrm{g}_{2}^{*}}{\partial \mathrm{V}_{3}} & \frac{\partial \mathrm{g}_{3}^{*}}{\partial \mathrm{V}_{3}} \\
\hdashline \frac{\partial \mathrm{g}_{1}^{*}}{\partial \theta_{2}} & \frac{\partial \mathrm{g}_{2}^{*}}{\partial \theta_{2}} & \frac{\partial \mathrm{g}_{3}^{*}}{\partial \theta_{2}} \\
\frac{\partial \mathrm{g}_{1}^{*}}{\partial \theta_{3}} & \frac{\partial \mathrm{g}_{2}^{*}}{\partial \theta_{3}} & \frac{\partial \mathrm{g}_{3}^{*}}{\partial \theta_{3}}
\end{array}\right] \quad \operatorname{diag}\left(\mathrm{h}_{\mathrm{j}}\right)=\left[\begin{array}{cccccccc}
\mathrm{h}_{1}^{*} & 0 & 0 & 0 & 0 & 0 & 0 & 0 \\
0 & \mathrm{~h}_{2}^{*} & 0 & 0 & 0 & 0 & 0 & 0 \\
0 & 0 & \mathrm{~h}_{3}^{*} & 0 & 0 & 0 & 0 & 0 \\
0 & 0 & 0 & \mathrm{~h}_{4}^{*} & 0 & 0 & 0 & 0 \\
0 & 0 & 0 & 0 & \mathrm{~h}_{5}^{*} & 0 & 0 & 0 \\
0 & 0 & 0 & 0 & 0 & \mathrm{~h}_{6}^{*} & 0 & 0 \\
0 & 0 & 0 & 0 & 0 & 0 & \mathrm{~h}_{7}^{*} & 0 \\
0 & 0 & 0 & 0 & 0 & 0 & 0 & \mathrm{~h}_{8}^{*}
\end{array}\right]
$$




$$
\mathbf{M}=\left[\begin{array}{lllllll}
\mu_{1}^{*} & 0 & 0 & 0 & 0 & 0 & 0 \\
0 & \mu_{2}^{*} & 0 & 0 & 0 & 0 & 0 \\
0 & 0 & \mu_{3}^{*} & 0 & 0 & 0 & 0 \\
0 & 0 & 0 & \mu_{4}^{*} & 0 & 0 & 0 \\
0 & 0 & 0 & 0 & \mu_{5}^{*} & 0 & 0 \\
0 & 0 & 0 & 0 & 0 & \mu_{6}^{*} & 0 \\
0 & 0 & 0 & 0 & 0 & 0 & \mu_{7}^{*}
\end{array}\right]
$$

$$
\varepsilon=\left[\begin{array}{l}
\varepsilon_{2}^{\mathrm{P}} \\
\varepsilon_{3}^{\mathrm{P}} \\
\varepsilon_{3}^{\mathrm{Q}}
\end{array}\right]
$$

Onde numericamente temos:

$\nabla^{2} L^{*}=\left[\begin{array}{ccccc}8.0000 & 0.0000 & -8.5692 & 0.0000 & 0.0000 \\ 0.0000 & 7.7799 & -8.7497 & -0.0000 & 0.0000 \\ -8.569 & -8.7497 & 18.7669 & -0.0000 & 0.0000 \\ 0.0000 & -0.0000 & -0.0000 & 9.8404 & -9.8404 \\ 0.0000 & 0.0000 & 0.0000 & -9.8404 & 19.0193\end{array}\right]$

$\mathrm{H}^{*}=\left[\begin{array}{cccccccc}-1.000 & 1.0000 & 0.0000 & 0.0000 & 0.0000 & 0.0000 & 0.0000 & 0.0000 \\ 0.0000 & 0.0000 & -1.000 & 1.0000 & 0.0000 & 0.0000 & -12.145 & 12.1454 \\ 0.0000 & 0.0000 & 0.0000 & 0.0000 & -1.000 & 1.0000 & 11.6378 & -11.637 \\ 0.0000 & 0.0000 & 0.0000 & 0.0000 & 0.0000 & 0.0000 & 3.3594 & -3.3594 \\ 0.0000 & 0.0000 & 0.0000 & 0.0000 & 0.0000 & 0.0000 & -3.359 & 3.3594\end{array}\right]$

$$
\operatorname{diag}\left(\mathrm{h}_{\mathrm{j}}^{*}\right)=\left[\begin{array}{cccccccc}
-0.2712 & 0.0000 & 0.0000 & 0.0000 & 0.0000 & 0.0000 & 0.0000 & 0.0000 \\
0.0000 & -0.1288 & 0.0000 & 0.0000 & 0.0000 & 0.0000 & 0.0000 & 0.0000 \\
0.0000 & 0.0000 & -0.3247 & 0.0000 & 0.0000 & 0.0000 & 0.0000 & 0.0000 \\
0.0000 & 0.0000 & 0.0000 & -0.0753 & 0.0000 & 0.0000 & 0.0000 & 0.0000 \\
0.0000 & 0.0000 & 0.0000 & 0.0000 & -0.100 & 0.0000 & 0.0000 & 0.0000 \\
0.0000 & 0.0000 & 0.0000 & 0.0000 & 0.0000 & 0.0000 & 0.0000 & 0.0000 \\
0.0000 & 0.0000 & 0.0000 & 0.0000 & 0.0000 & 0.0000 & -0.0108 & 0.0000 \\
0.0000 & 0.0000 & 0.0000 & 0.0000 & 0.0000 & 0.0000 & 0.0000 & -0.9892
\end{array}\right]
$$




$$
G^{*}=\left[\begin{array}{lll}
0.000 & 4.1139 & 4.9067 \\
-6.0102 & 4.9734 & 9.5533 \\
3.3594 & -6.0000 & -14.0000 \\
-11.637 & 10.7442 & -5.5933 \\
11.6378 & -16.000 & 10.0000
\end{array}\right]
$$

$$
\mathrm{M}=\left[\begin{array}{llllllll}
0.000 & 0.000 & 0.000 & 0.000 & 0.000 & 0.000 & 0.000 & 0.000 \\
0.000 & 0.000 & 0.000 & 0.000 & 0.000 & 0.000 & 0.000 & 0.000 \\
0.000 & 0.000 & 0.000 & 0.000 & 0.000 & 0.000 & 0.000 & 0.000 \\
0.000 & 0.000 & 0.000 & 0.000 & 0.000 & 0.000 & 0.000 & 0.000 \\
0.000 & 0.000 & 0.000 & 0.000 & 0.000 & 0.000 & 0.000 & 0.000 \\
0.000 & 0.000 & 0.000 & 0.000 & 0.000 & 0.252 & 0.000 & 0.000 \\
0.000 & 0.000 & 0.000 & 0.000 & 0.000 & 0.000 & 0.000 & 0.000 \\
0.000 & 0.000 & 0.000 & 0.000 & 0.000 & 0.000 & 0.000 & 0.000
\end{array}\right]
$$

A seguir está representada a matriz $\mathrm{S}^{*}$ com todos os elementos. Notamos que a matriz é esparsa e simétrica de posição. Essas características da matriz são exploradas pela sub-rotina MA28.

\footnotetext{
$S^{*}=\left[\begin{array}{cccccccccccccccc}8.0000 & 0.0000 & -8.5692 & 0.0000 & 0.0000 & -1.0000 & 1.0000 & 0.0000 & 0.0000 & 0.0000 & 0.0000 & 0.0000 & 0.0000 & 0.0000 & 4.1139 & 4.9067 \\ 0.0000 & 7.7799 & -8.7497 & -0.0000 & 0.0000 & 0.0000 & 0.0000 & -1.000 & 1.0000 & 0.0000 & 0.0000 & -12.1454 & 12.1454 & -6.0102 & 4.9734 & 9.5533 \\ -8.5692 & -8.7497 & 18.7669 & -0.0000 & 0.0000 & 0.0000 & 0.0000 & 0.0000 & 0.0000 & -1.000 & 1.0000 & 11.6378 & -116378 & 3.3594 & -6.000 & -14.000 \\ 0.0000 & -0.0000 & -0.0000 & 9.8404 & -9.8404 & 0.0000 & 0.0000 & 0.0000 & 0.0000 & 0.0000 & 0.0000 & 3.3594 & -3.3594 & -11.637 & 10.744 & -5.5933 \\ 0.0000 & 0.0000 & 0.0000 & -9.8404 & 19.0193 & 0.0000 & 0.0000 & 0.0000 & 0.0000 & 0.0000 & 0.0000 & -3.3594 & 3.3594 & 11.637 & -16.000 & 10.000 \\ 0.0000 & 0.0000 & 0.0000 & 0.0000 & 0.0000 & -0.2712 & 0.0000 & 0.0000 & 0.0000 & 0.0000 & 0.0000 & 0.0000 & 0.0000 & 0.0000 & 0.0000 & 0.0000 \\ 0.0000 & 0.0000 & 0.0000 & 0.0000 & 0.0000 & 0.0000 & -0.1288 & 0.0000 & 0.0000 & 0.0000 & 0.0000 & 0.0000 & 0.0000 & 0.0000 & 0.0000 & 0.0000 \\ 0.0000 & 0.0000 & 0.0000 & 0.0000 & 0.0000 & 0.0000 & 0.0000 & -0.324 & 0.0000 & 0.0000 & 0.0000 & 0.0000 & 0.0000 & 0.0000 & 0.0000 & 0.0000 \\ 0.0000 & 0.0000 & 0.0000 & 0.0000 & 0.0000 & 0.0000 & 0.0000 & 0.0000 & -0.0753 & 0.0000 & 0.0000 & 0.0000 & 0.0000 & 0.0000 & 0.0000 & 0.0000 \\ 0.0000 & 0.0000 & 0.0000 & 0.0000 & 0.0000 & 0.0000 & 0.0000 & 0.0000 & 0.0000 & -0.100 & 0.0000 & 0.0000 & 0.0000 & 0.0000 & 0.0000 & 0.0000 \\ 0.0000 & 0.0000 & 0.0000 & 0.2524 & 0.0000 & 0.0000 & 0.0000 & 0.0000 & 0.0000 & 0.0000 & 0.0000 & 0.0000 & 0.0000 & 0.0000 & 0.0000 & 0.0000 \\ 0.0000 & 0.0000 & 0.0000 & 0.0000 & 0.0000 & 0.0000 & 0.0000 & 0.0000 & 0.0000 & 0.0000 & 0.0000 & -0.0108 & 0.0000 & 0.0000 & 0.0000 & 0.0000 \\ 0.0000 & 0.0000 & 0.0000 & 0.0000 & 0.0000 & 0.0000 & 0.0000 & 0.0000 & 0.0000 & 0.0000 & 0.0000 & 0.0000 & -0.9892 & 0.0000 & 0.0000 & 0.0000 \\ 0.0000 & -6.0102 & 3.3594 & -11.6378 & 11.6378 & 0.0000 & 0.0000 & 0.0000 & 0.0000 & 0.0000 & 0.0000 & 0.0000 & 0.0000 & 0.0000 & 0.0000 & 0.0000 \\ 4.1139 & 4.9734 & -6.0000 & 10.7442 & -16.0000 & 0.0000 & 0.0000 & 0.0000 & 0.0000 & 0.0000 & 0.0000 & 0.0000 & 0.0000 & 0.0000 & 0.0000 & 0.0000 \\ 4.9067 & 9.5533 & -14.0000 & -5.5933 & 10.0000 & 0.0000 & 0.0000 & 0.0000 & 0.0000 & 0.0000 & 0.0000 & 0.0000 & 0.0000 & 0.0000 & 0.0000 & 0.0000\end{array}\right]$
}

Tendo a matriz $\mathrm{S}^{*}$, podemos estimar novas soluções depois de realizadas perturbações no sistema. Para exemplificar, realizamos uma aplicação em que 
aumentamos a carga da barra de carga 3 em 5\%, o que corresponde a uma perturbação de $\varepsilon_{3}^{\mathrm{P}}=10,00 \mathrm{MWe} \varepsilon_{3}^{\mathrm{Q}}=5,00 \mathrm{MVAr}$ nas potências ativa e reativa, respectivamente, como mostrado na Tabela 6.5 .

TABELA 6.5 - Alterações nos parâmetros da barras 3

\begin{tabular}{|c|c|c|c|}
\hline \hline Barra k & Tipo & $\mathrm{P}_{\mathrm{k}}(\mathrm{MW})$ & $\mathrm{Q}_{\mathrm{k}}(\mathrm{MVAr})$ \\
\hline \hline 1 & Slack & --- & --- \\
\hline 2 & PV & 170,0 & --- \\
\hline 3 & PQ & $\mathbf{- 2 1 0 , 0}$ & $\mathbf{- 1 0 5 , 0}$ \\
\hline
\end{tabular}

Considerando-se a potência de base 100 MVA, o vetor E em p.u. é representado por:

$\mathrm{E}=\left[\begin{array}{l}0.0000 \\ 0.0000 \\ 0.0000 \\ 0.0000 \\ 0.0000 \\ 0.0000 \\ 0.0000 \\ 0.0000 \\ 0.0000 \\ 0.0000 \\ 0.0000 \\ 0.0000 \\ 0.0000 \\ 0.0000 \\ 0.1000 \\ 0.0500\end{array}\right]$

Utilizando a sub-rotina MA28, obtemos o vetor resposta, $\mathrm{R}_{\mathrm{S}}$, do sistema. Tendo o vetor $R_{S}$, a solução do problema perturbado é obtida, simplesmente, 
atualizando-se as variáveis, ou seja, somando o vetor $R_{S}$ ao vetor das variáveis ótimas do sistema, como mostrado em (6.5).

$$
\left[\begin{array}{l}
\mathrm{x}(\varepsilon) \\
\gamma(\varepsilon) \\
\lambda(\varepsilon)
\end{array}\right]=\left[\begin{array}{l}
\mathrm{x}^{*} \\
\gamma^{*} \\
\lambda^{*}
\end{array}\right]-\left[\begin{array}{l}
\mathrm{R}_{\mathrm{S}} \\
\end{array}\right]
$$

Numericamente temos:

$$
\left[\begin{array}{l}
x_{1}(\varepsilon) \\
x_{2}(\varepsilon) \\
x_{3}(\varepsilon) \\
\theta_{2}(\varepsilon) \\
\theta_{3}(\varepsilon) \\
\gamma_{1}(\varepsilon) \\
\gamma_{2}(\varepsilon) \\
\gamma_{3}(\varepsilon) \\
\gamma_{4}(\varepsilon) \\
\gamma_{5}(\varepsilon) \\
\gamma_{6}(\varepsilon) \\
\gamma_{7}(\varepsilon) \\
\gamma_{8}(\varepsilon) \\
\lambda_{1}(\varepsilon) \\
\lambda_{2}(\varepsilon) \\
\lambda_{3}(\varepsilon)
\end{array}\right]=\left[\begin{array}{l}
1.0711 \\
1.1246 \\
1.0000 \\
0.0764 \\
-0.0229 \\
0.0000 \\
0.0000 \\
0.0000 \\
0.0000 \\
0.2524 \\
0.0000 \\
0.0000 \\
0.0000 \\
0.0275 \\
-0.0793 \\
-0.0499
\end{array}\right]-\left[\begin{array}{l}
-0.0121 \\
-0.0027 \\
0.0000 \\
0.0112 \\
0.0098 \\
0.0000 \\
0.0000 \\
0.0000 \\
0.0000 \\
0.0246 \\
0.0000 \\
0.0000 \\
0.0000 \\
0.0179 \\
0.0198 \\
0.0032
\end{array}\right]
$$

As Tabelas 6.6 e 6.7 mostram a solução do problema perturbado após a utilização da metodologia SFPO. 
TABELA 6.6 - Variáveis primais e geração de potência reativa após a perturbação

\begin{tabular}{|c|c|c|c||}
\hline Barra $\mathrm{k}$ & $\mathrm{V}_{\mathrm{k}}$ (p.u.) & $\theta_{\mathrm{k}}$ (graus) & $\mathrm{Q}$ (MVAr) \\
\hline \hline 1 & 1,0832 & 0 & 28,9 \\
\hline 2 & 1,1273 & 3,73 & 109,9 \\
\hline 3 & 1,0000 & $-1,87$ & --- \\
\hline \multicolumn{2}{|c|}{ Perdas (MW) } & $14,06 \mathrm{MW}$ \\
\hline
\end{tabular}

TABELA 6.7 - Variáveis duais após a perturbação

\begin{tabular}{|c|c|c|c|c|c|c|}
\hline Barra k & $\begin{array}{c}\mu \text { (limite } \\
\text { superior } \\
\text { de } \mathrm{V}_{\mathrm{k}} \text { ) }\end{array}$ & $\begin{array}{c}\mu \text { (limite } \\
\text { inferior de } \\
\mathrm{V}_{\mathrm{k}} \text { ) }\end{array}$ & $\begin{array}{c}\mu \text { (limite } \\
\text { superior } \\
\left.\text { de } \mathrm{Q}_{\mathrm{k}}\right)\end{array}$ & $\begin{array}{c}\mu \text { (limite } \\
\text { inferior de } \\
\mathrm{Q}_{\mathrm{k}} \text { ) }\end{array}$ & $\begin{array}{c}\lambda \text { (balanço } \\
\mathrm{da} \\
\text { potência } \\
\text { ativa) }\end{array}$ & $\begin{array}{c}\lambda \text { (balanço } \\
\mathrm{da} \\
\text { potência } \\
\text { reativa) }\end{array}$ \\
\hline 1 & 0,0000 & 0,0000 & --- & --- & --- & --- \\
\hline 2 & 0,0000 & 0,0000 & 0,0000 & 0,0000 & 0,0096 & --- \\
\hline 3 & 0,2278 & 0,0000 & --- & --- & $-0,0991$ & $-0,0531$ \\
\hline
\end{tabular}

Os erros nas equações de balanço foram menores que $7 \times 10^{-4}$ p.u., o que caracteriza um ponto de operação.

\subsection{EXEMPLO 2: SISTEMA IEEE 14 BARRAS}

Para o sistema IEEE 14 barras, representado pela Figura 6.2, fazemos um acréscimo de carga em todas as barras de carga de $2 \%$ em $2 \%$, mantendo o fator de potência constante, até um total de $20 \%$. Para cada acréscimo de carga, utilizamos o programa SFPO para encontrar um novo ponto de operação. Os resultados encontrados pelo programa SFPO foram comparados com os resultados obtidos pelo programa de FPO para a mesma situação de carga. Esse teste tem como finalidade validar a metodologia SFPO. A seguir, apresentamos as Figuras de comparação entre os resultados obtidos pelos programas de SFPO e FPO. As Figuras contêm as curvas das perdas de potência ativa na transmissão, dos ângulos de fase e das magnitudes das tensões de todas as barras, geração de reativos nas barras de geração e a curva de 
maior erro das equações de balanço de potência ativa e reativa em relação a porcentagem de perturbação.

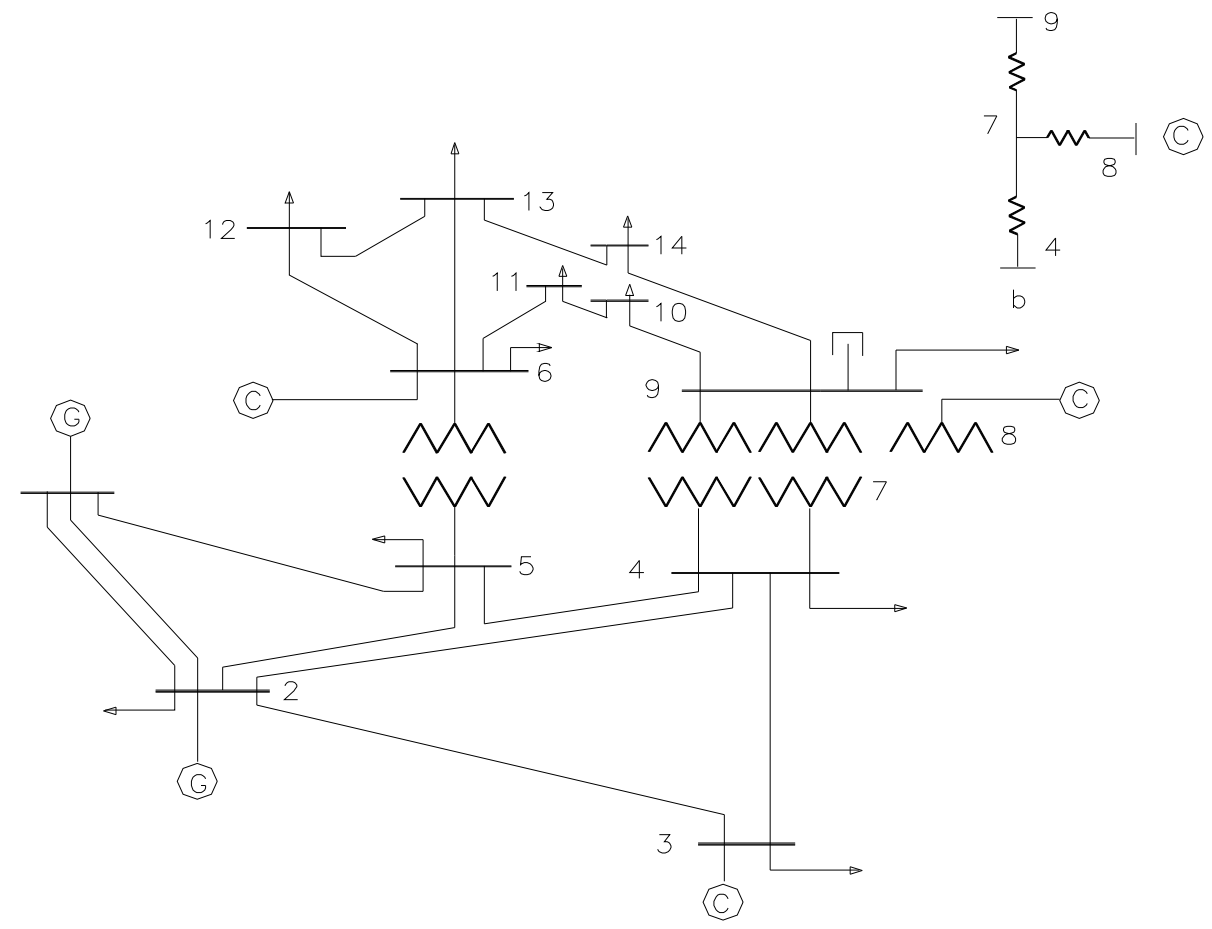

Figura 6.2 - Sistema 14 barras

As curvas a seguir foram impressas nas cores azul escuro e rosa, representando respectivamente os resultados obtidos através do programa de FPO e do programa SFPO. Todos os gráficos mostram o acréscimo de carga de $0 \%$ até $20 \%$ nas barras de carga.

A Figura 6.3 mostra o valor das perdas de potência ativa na transmissão. As perdas mantiveram-se praticamente no mesmo valor para os dois casos, FPO e SFPO, em todos os níveis de carga. 


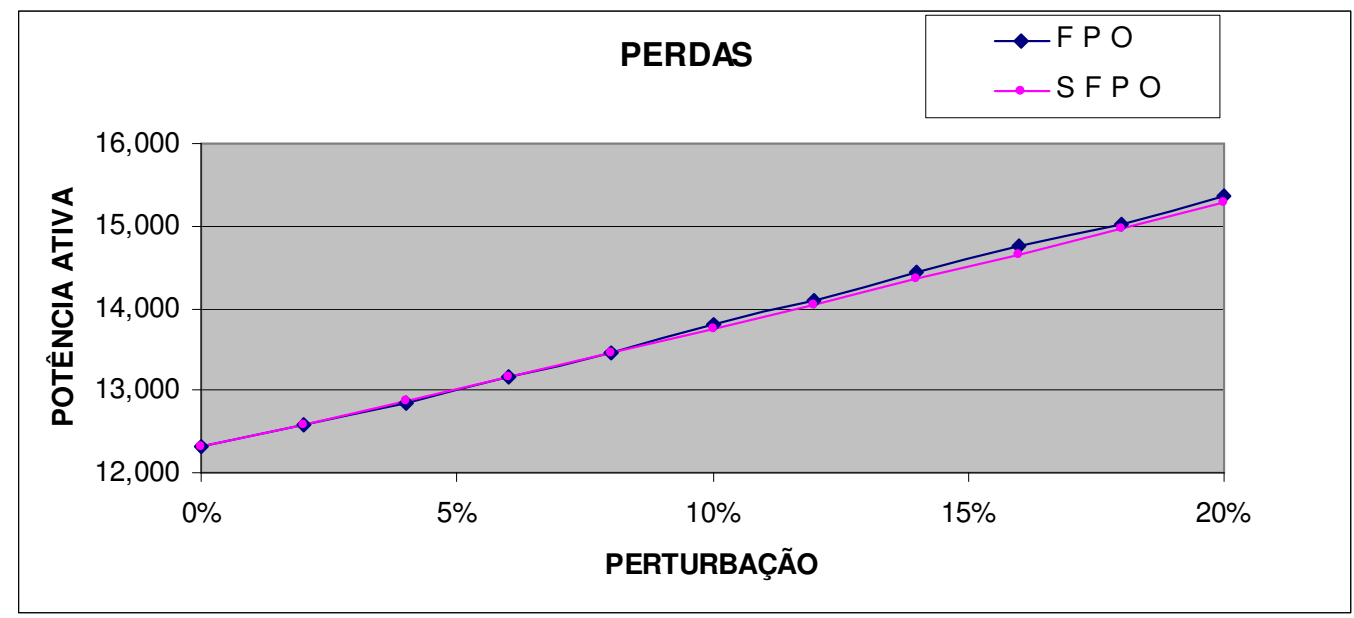

Figura 6.3 - Perdas de potência ativa na transmissão

A seguir apresentaremos as curvas dos ângulos de fase das barras do sistema. A curva da barra slack, não está apresentada, pelo fato de ser referência angular, e, portanto, não variar.

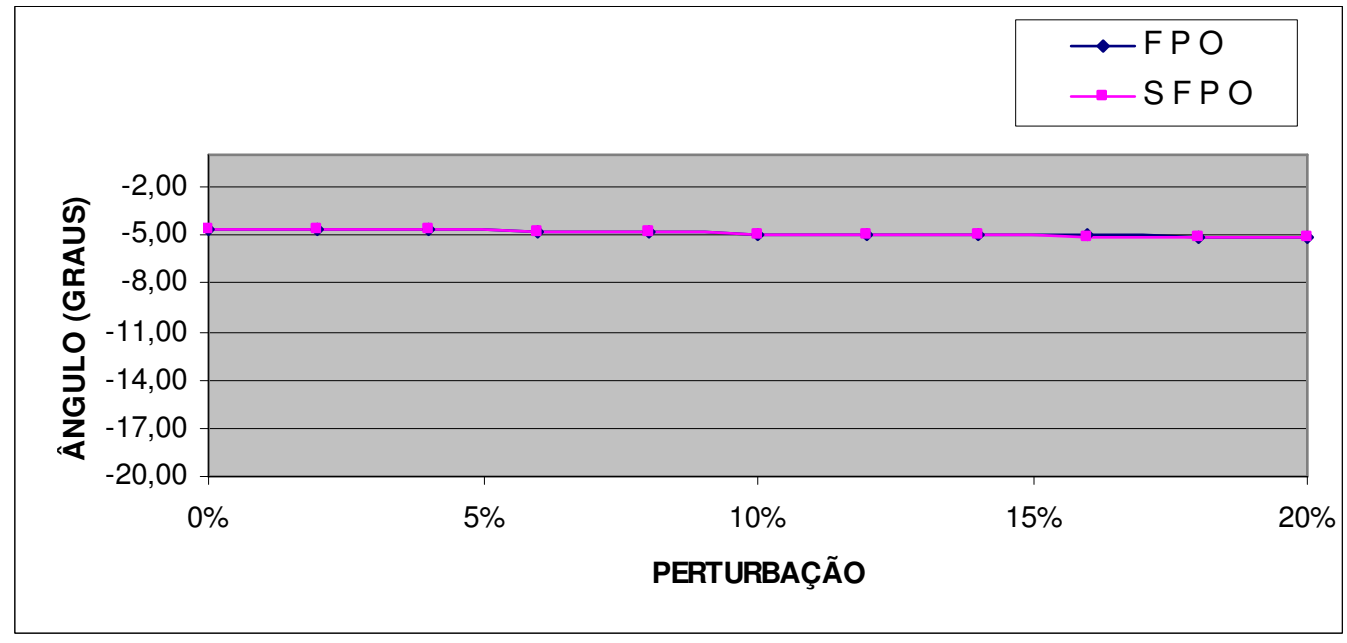

Figura 6.4 - Ângulo de fase na barra 2 


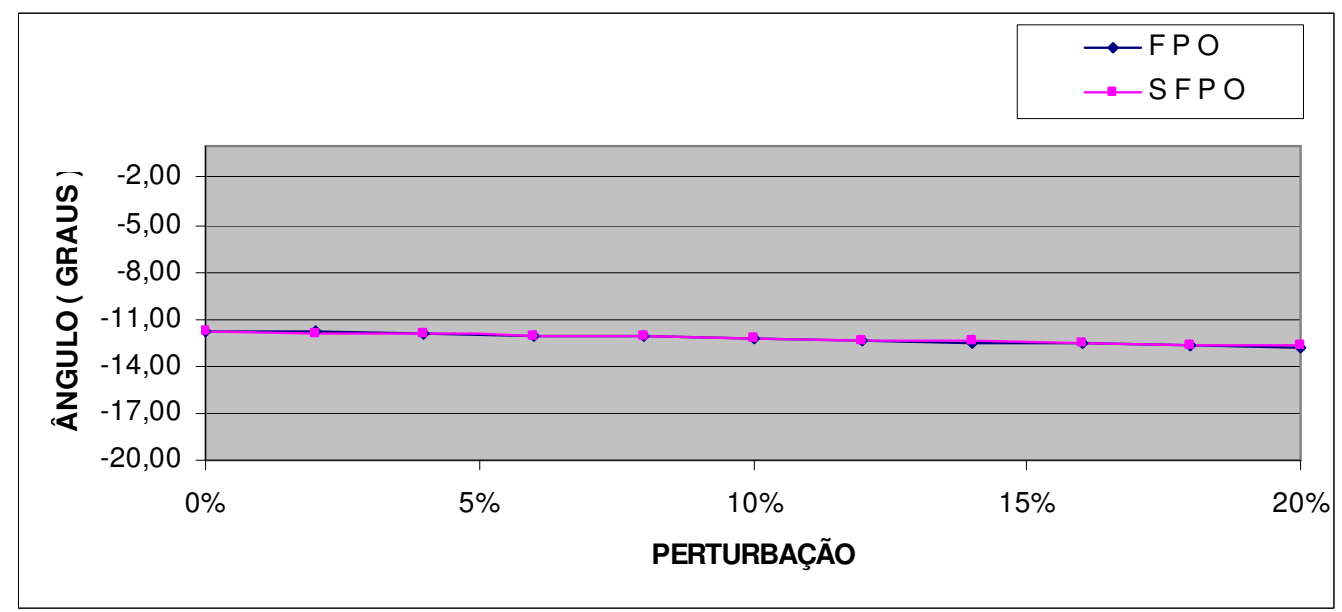

Figura 6.5 - Ângulo de fase na barra 3

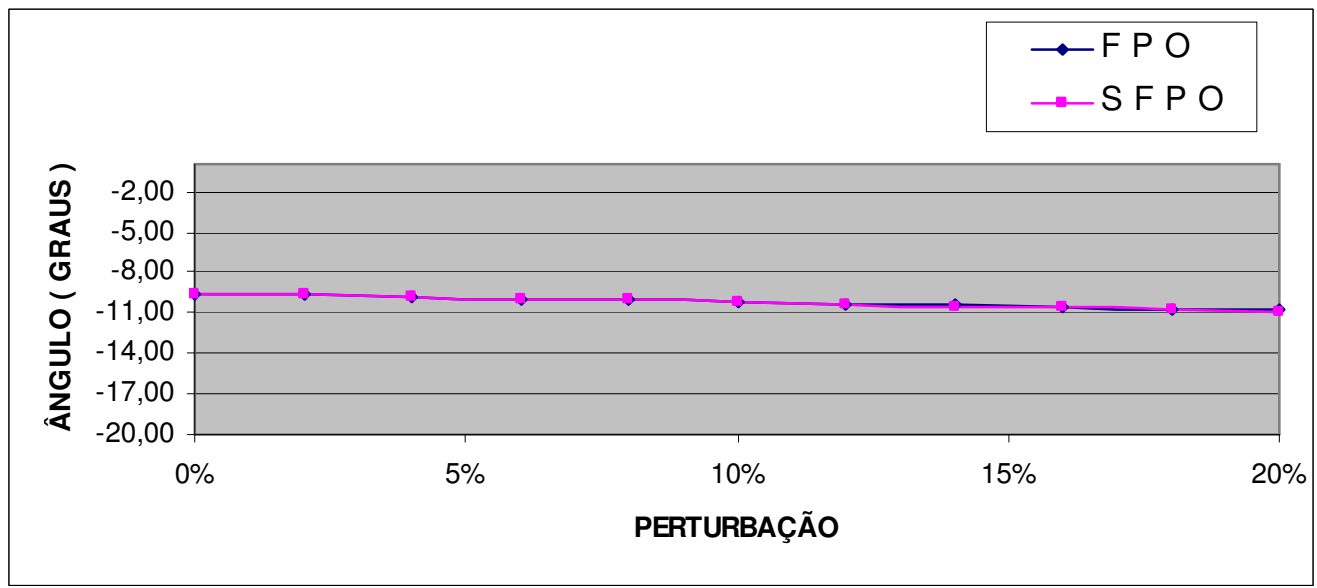

Figura 6.6 - Ângulo de fase na barra 4

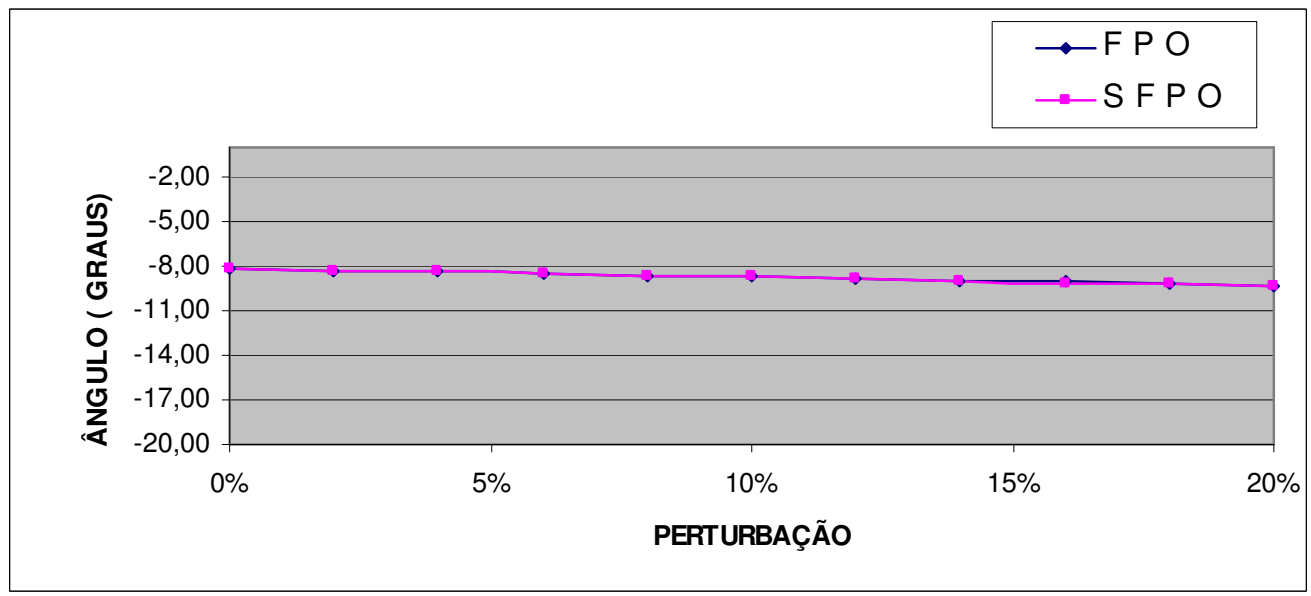

Figura 6.7 - Ângulo de fase na barra 5 


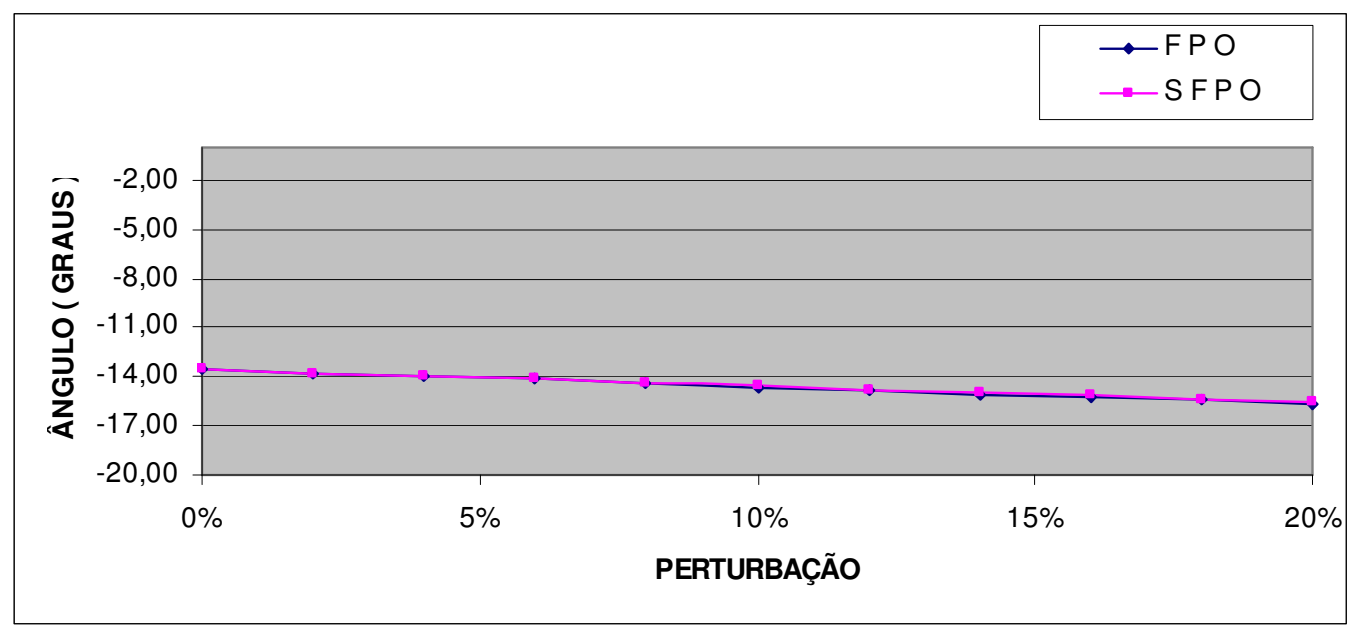

Figura 6.8 - Ângulo de fase na barra 6

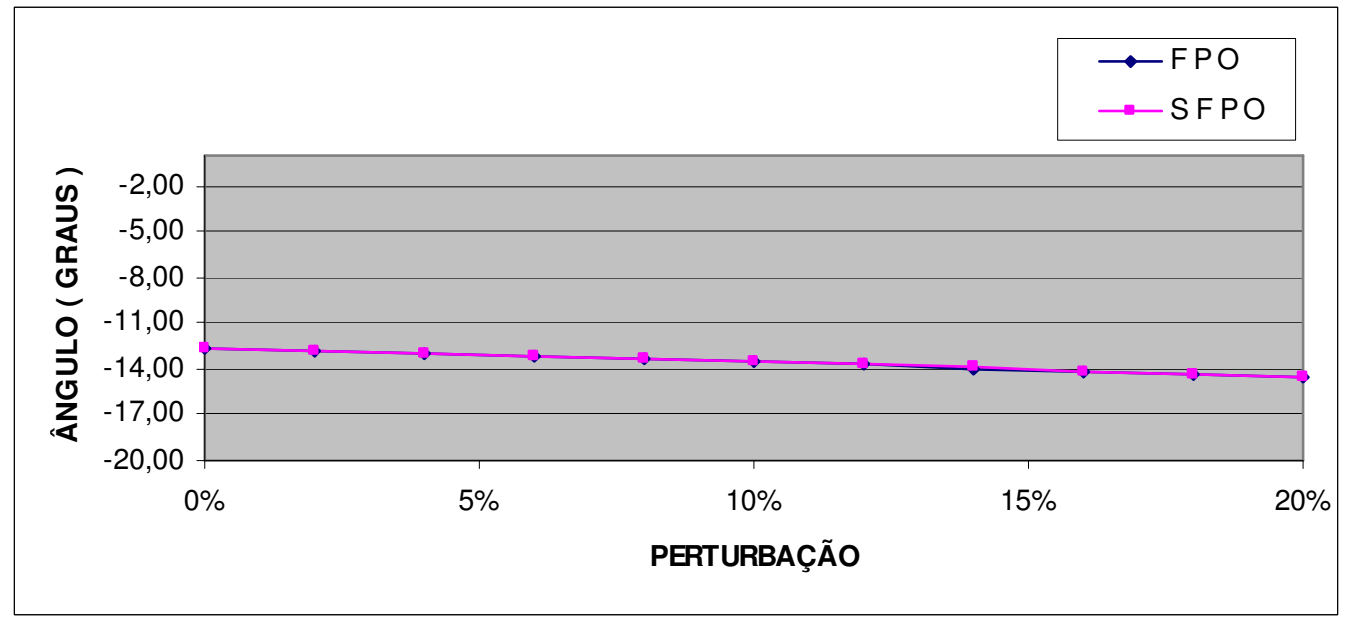

Figura 6.9 - Ângulo de fase na barra 7

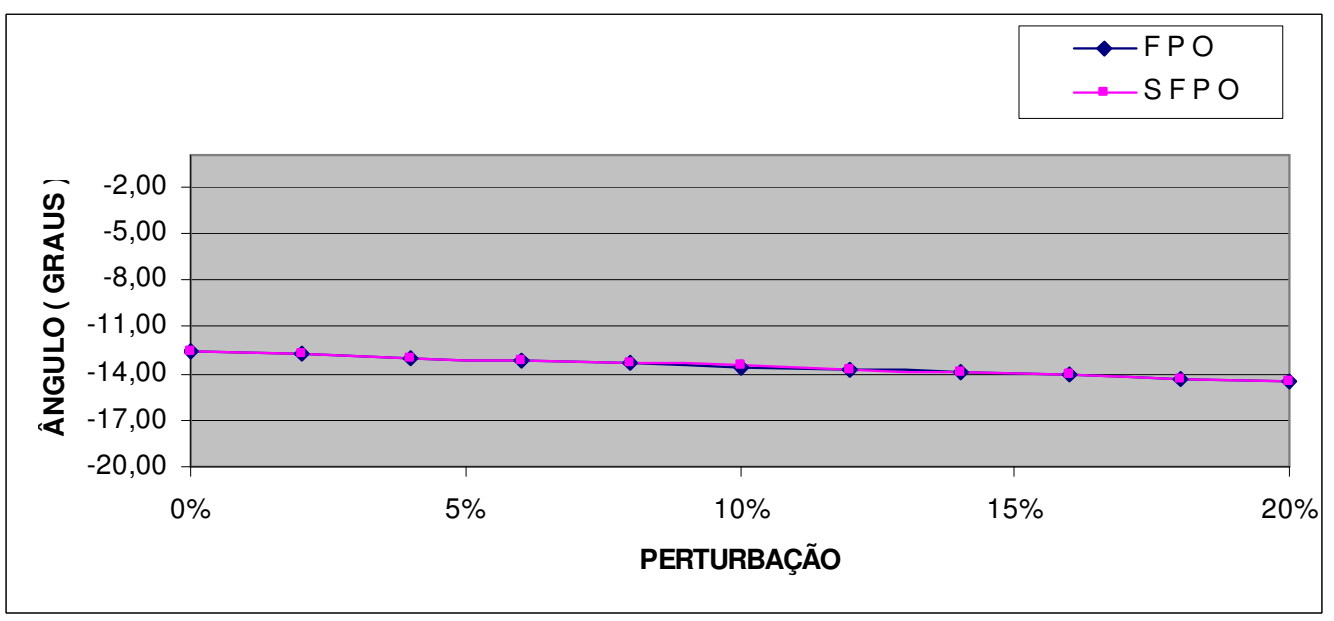

Figura 6.10 - Ângulo de fase na barra 8 


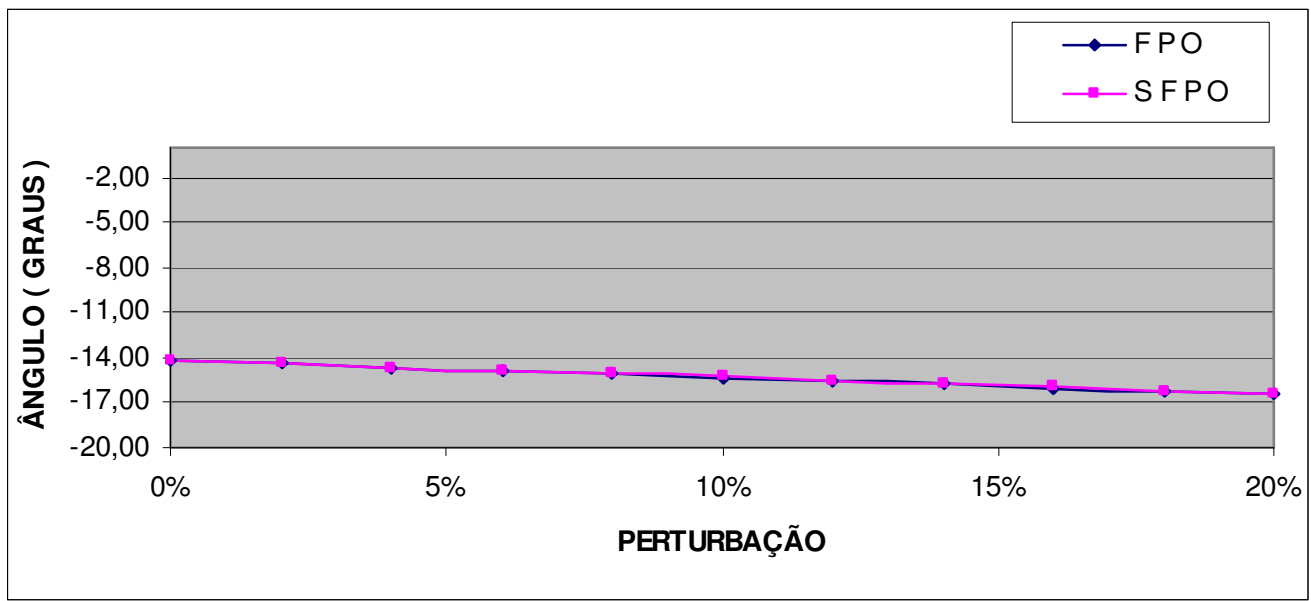

Figura 6.11 - Ângulo de fase na barra 9

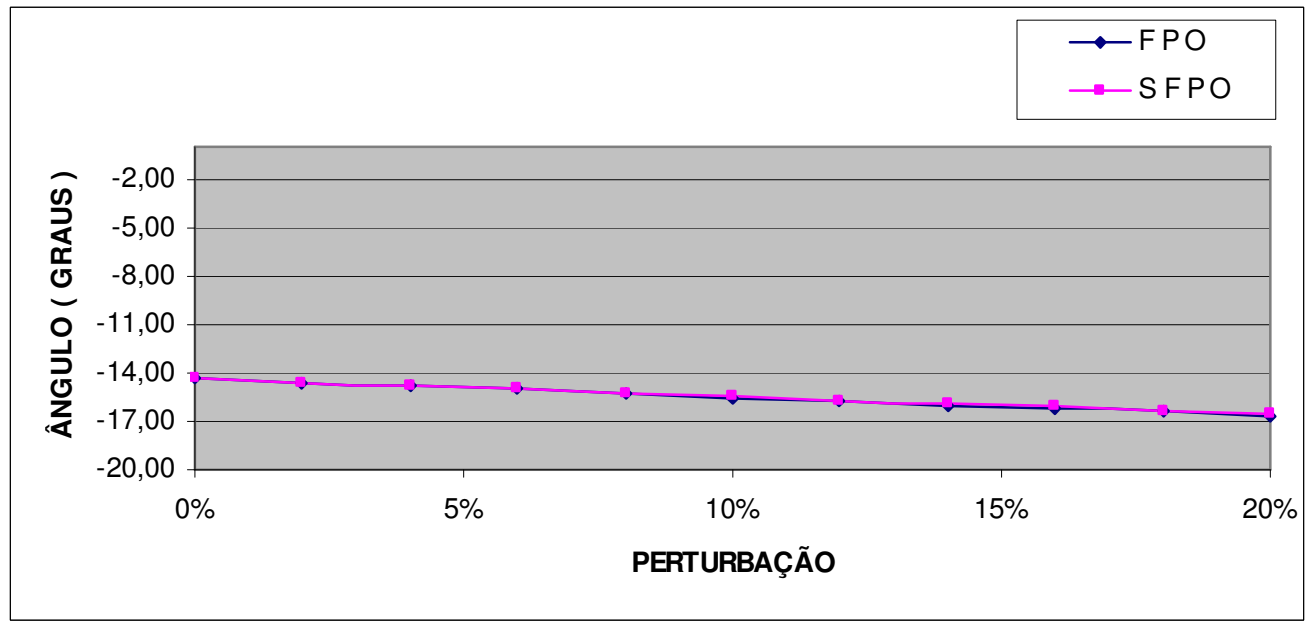

Figura 6.12 - Ângulo de fase na barra 10

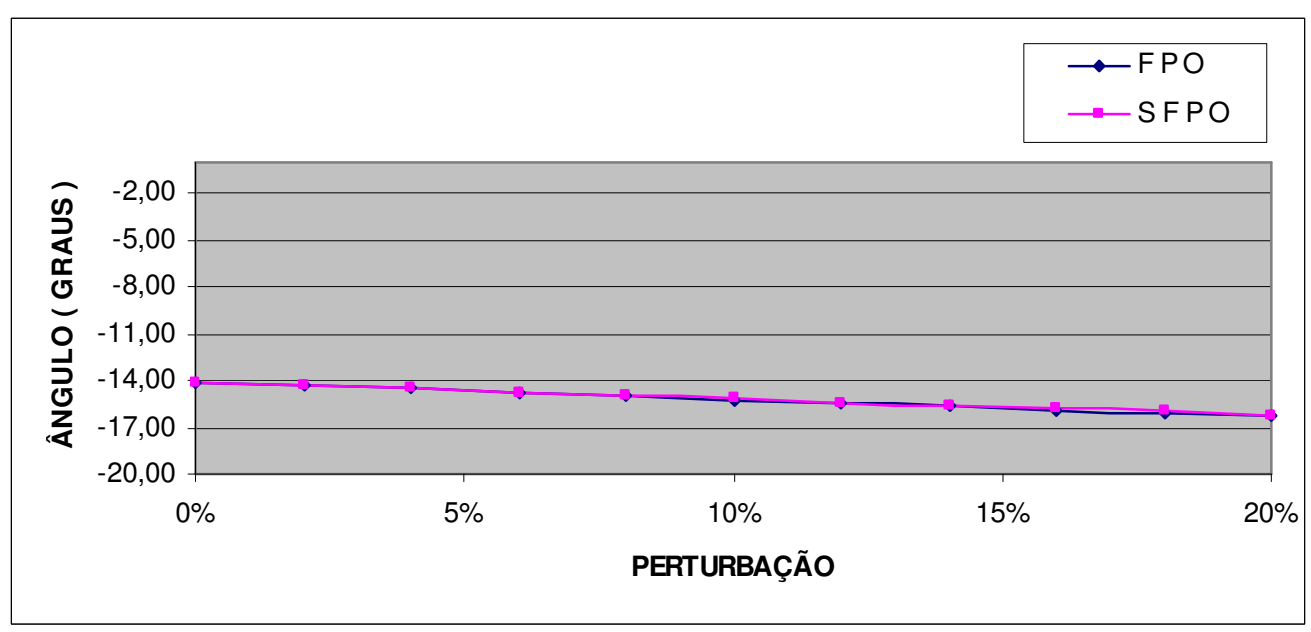

Figura 6.13 - Ângulo de fase na barra 11 


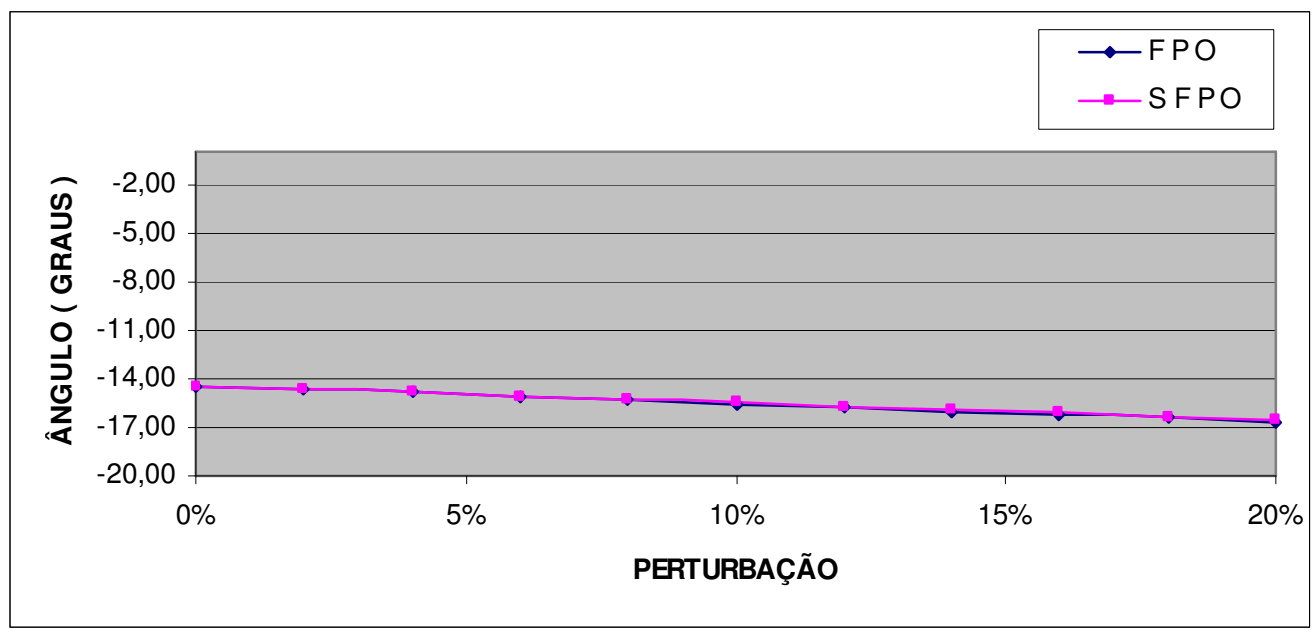

Figura 6.14 - Ângulo de fase na barra 12

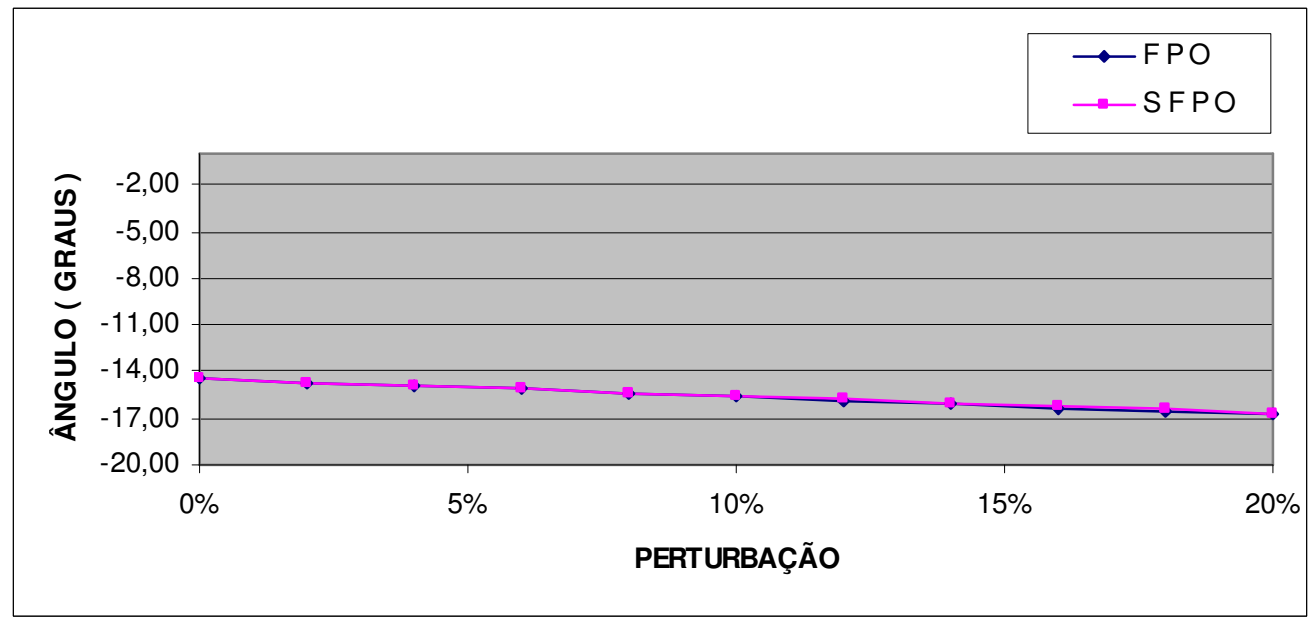

Figura 6.15 - Ângulo de fase na barra 13

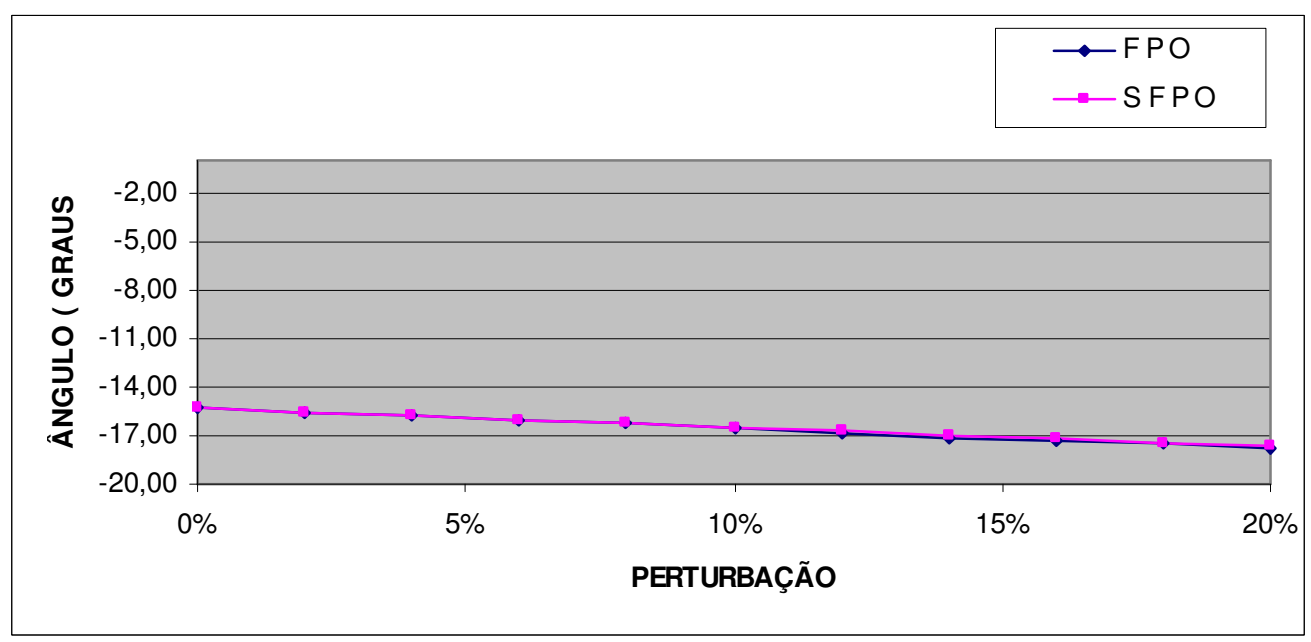

Figura 6.16 - Ângulo de fase na barra 14 
Vimos no conjunto de Figuras anteriores, que relacionam o ângulo de fase com a perturbação no sistema, a variação do ângulo nas barras do sistema. Os ângulos obtidos através do programa SFPO mantiveram-se muito próximos dos obtidos pelo FPO, mesmo para perturbações próximas a 20\%. O comportamento dos ângulos mostra que o programa de SFPO também pode fazer parte de um estudo de uma metodologia voltada para um fluxo de potência ótimo DC.

A seguir apresentaremos as curvas da magnitude da tensão em todas as barras do sistema.

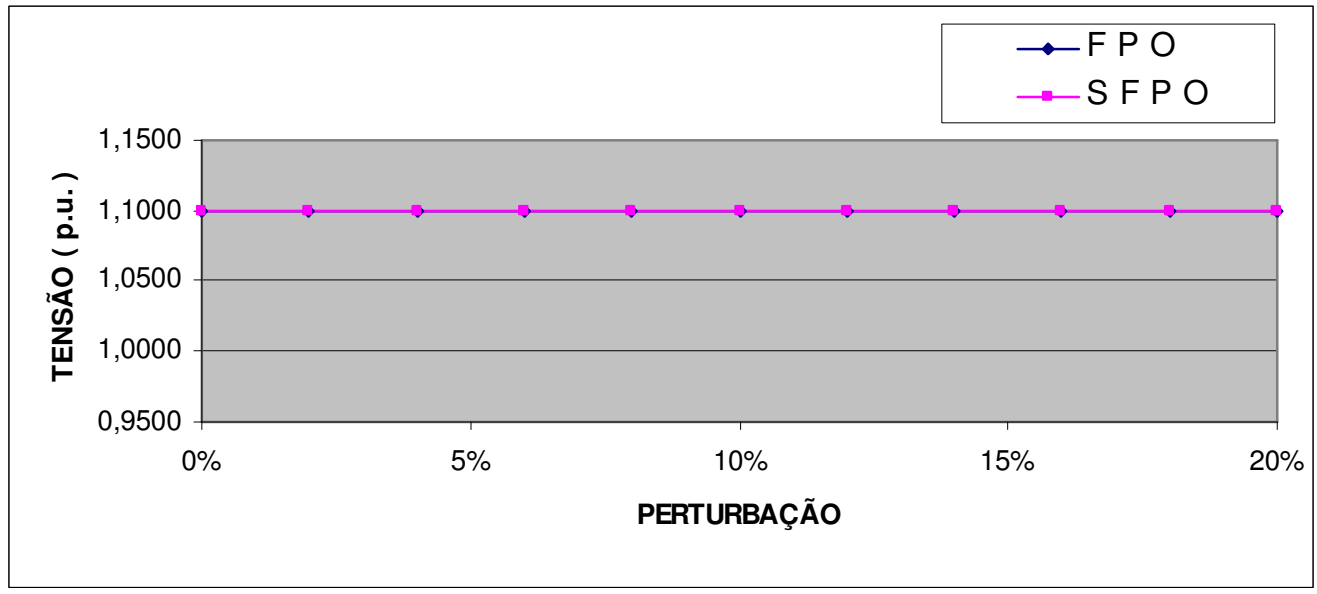

Figura 6.17 - Magnitude da tensão na barra 1

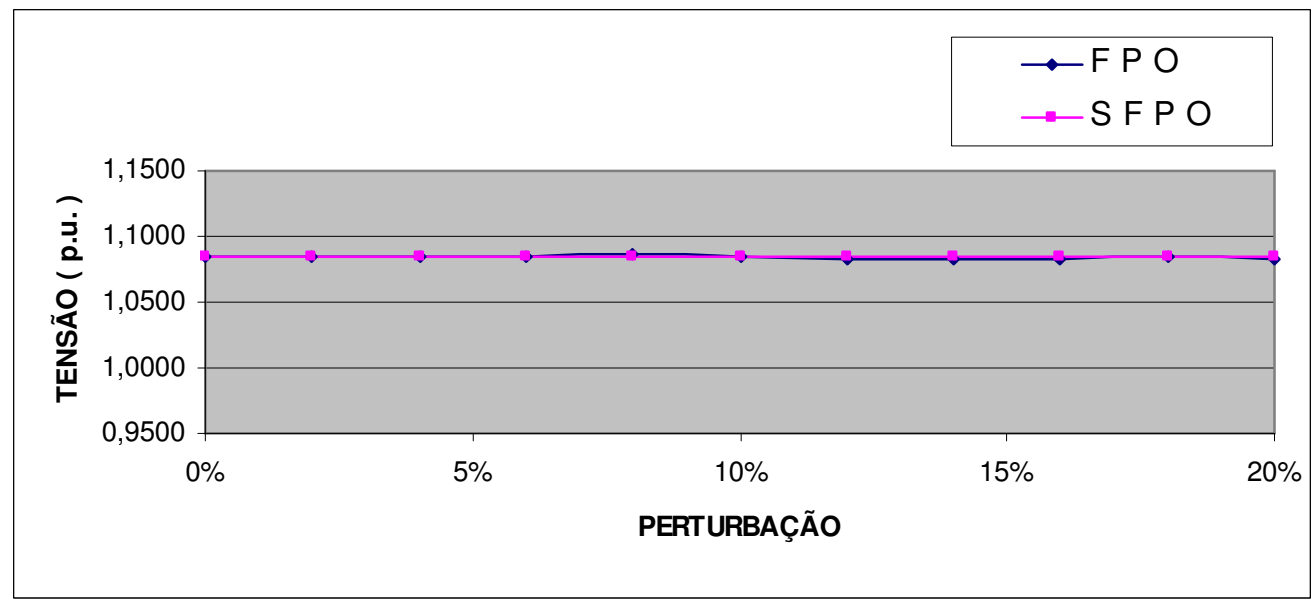

Figura 6.18 - Magnitude da tensão na barra 2 


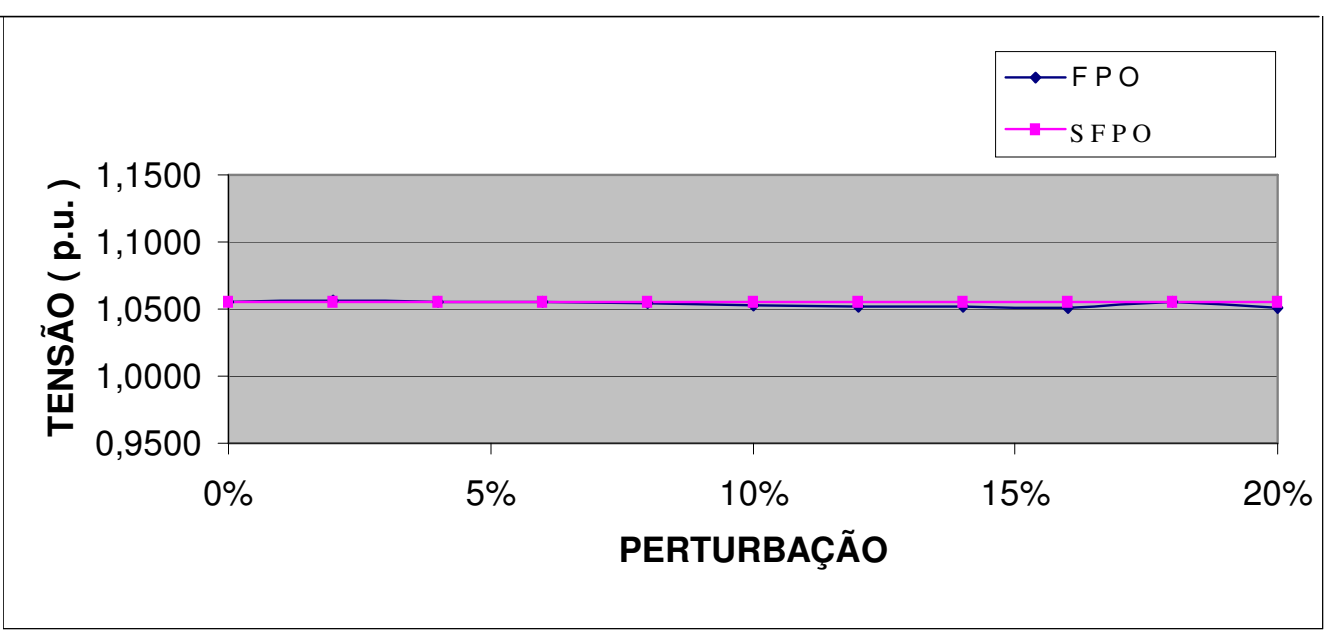

Figura 6.19 - Magnitude da tensão na barra 3

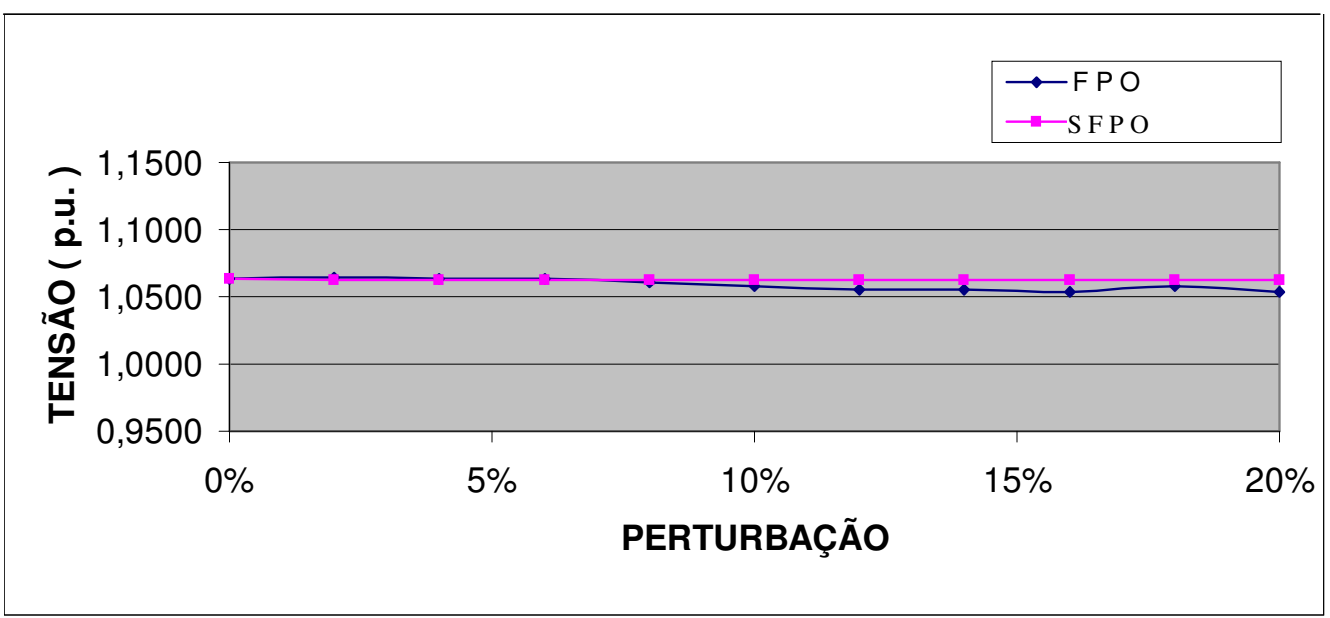

Figura 6.20 - Magnitude da tensão na barra 4

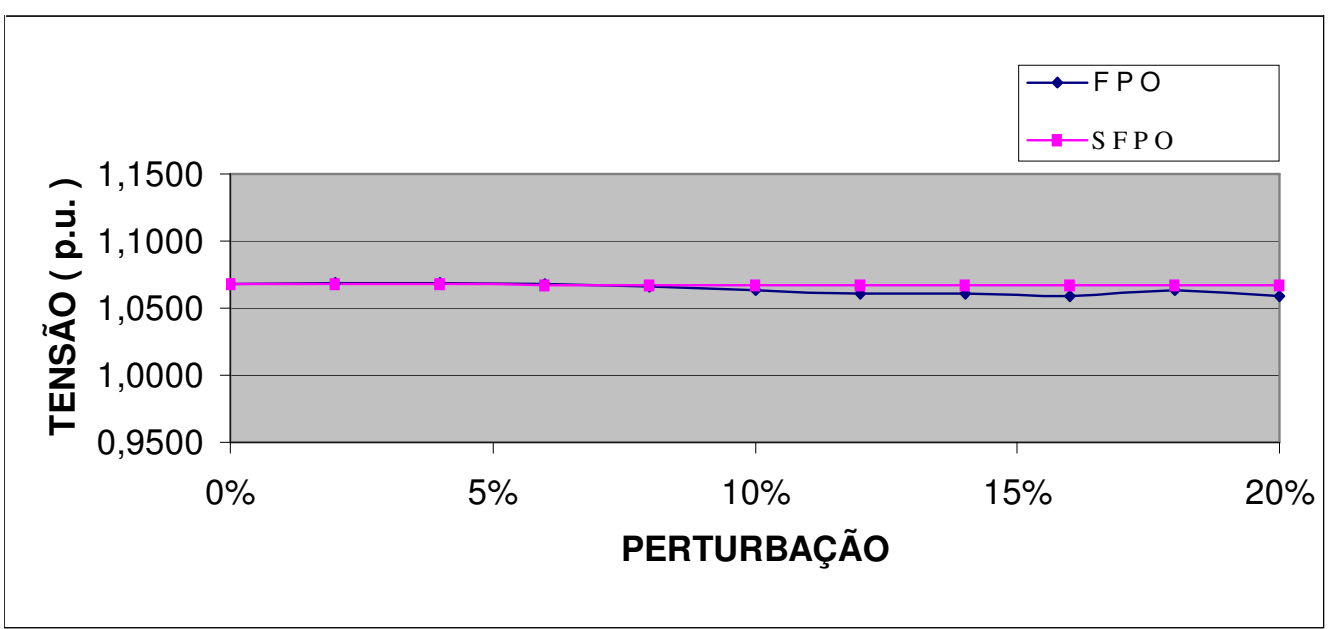

Figura 6.21 - Magnitude da tensão na barra 5 


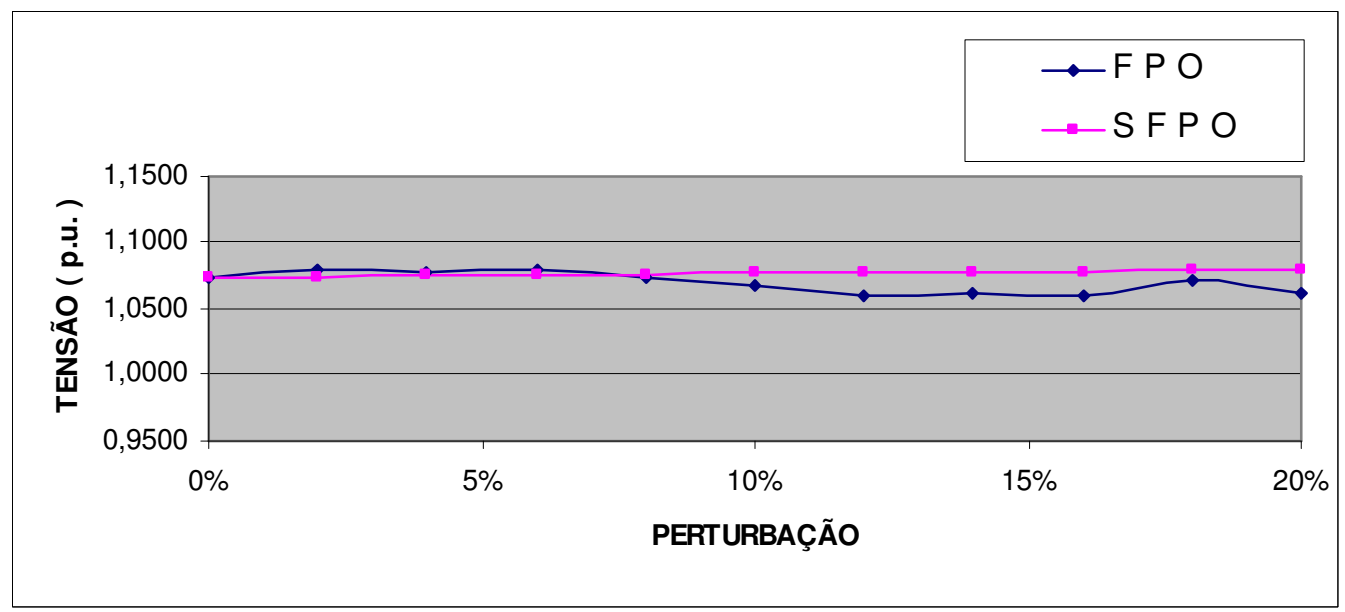

Figura 6.22 - Magnitude da tensão na barra 6

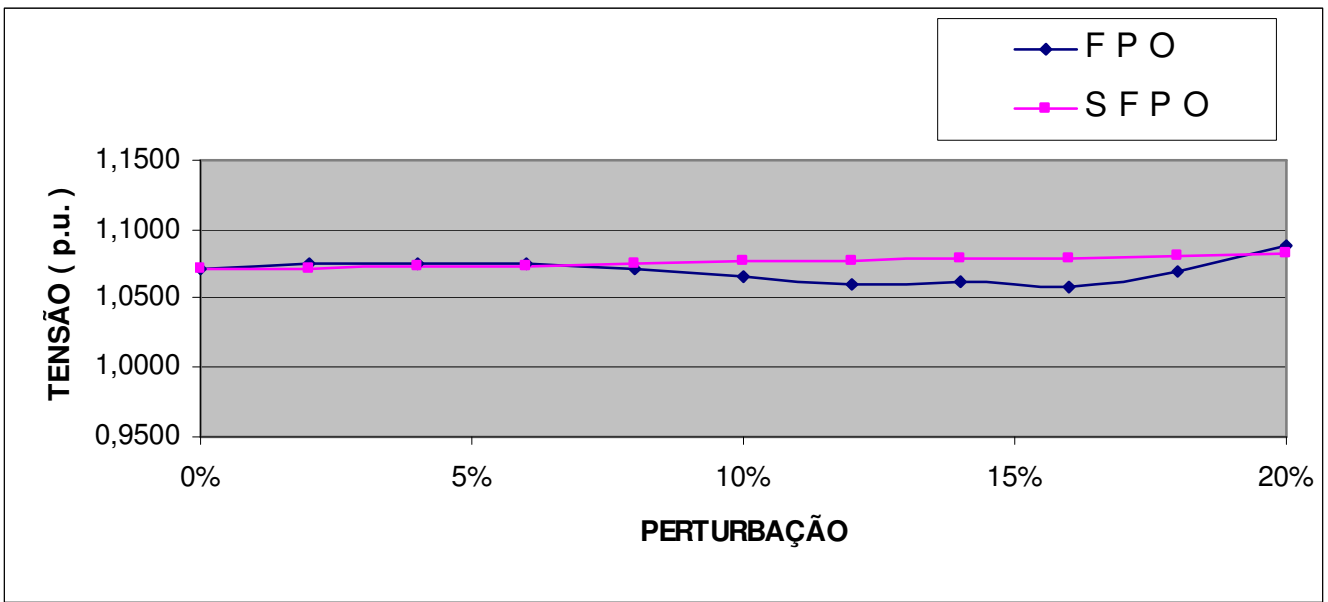

Figura 6.23 - Magnitude da tensão na barra 7

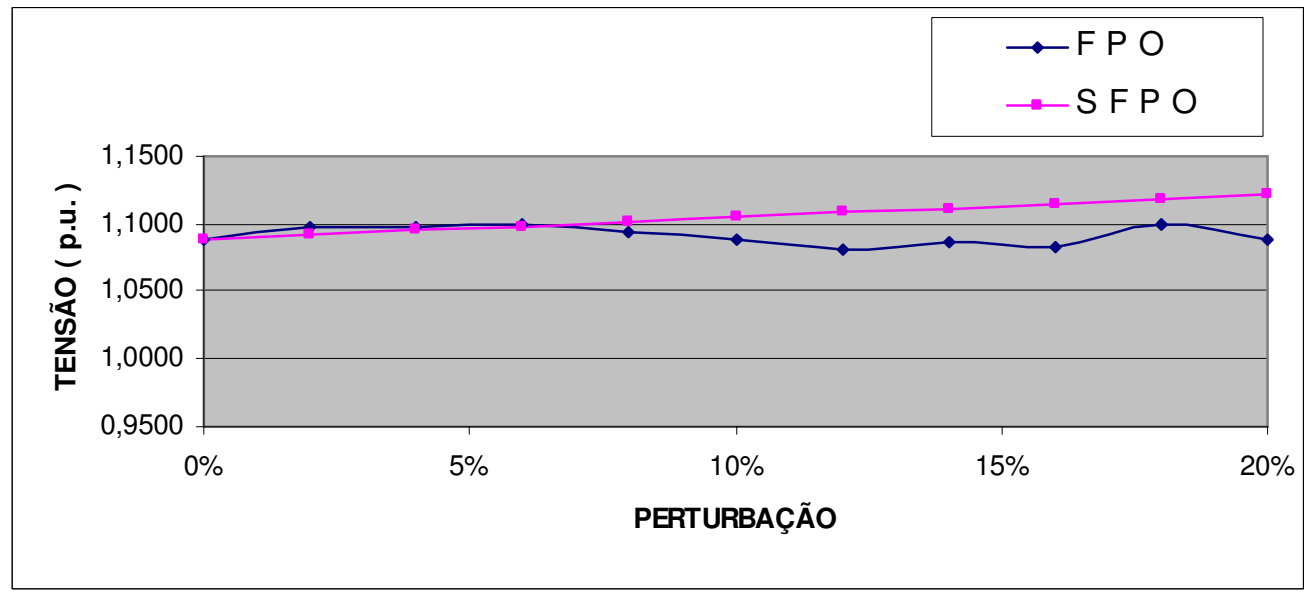

Figura 6.24 - Magnitude da tensão na barra 8 


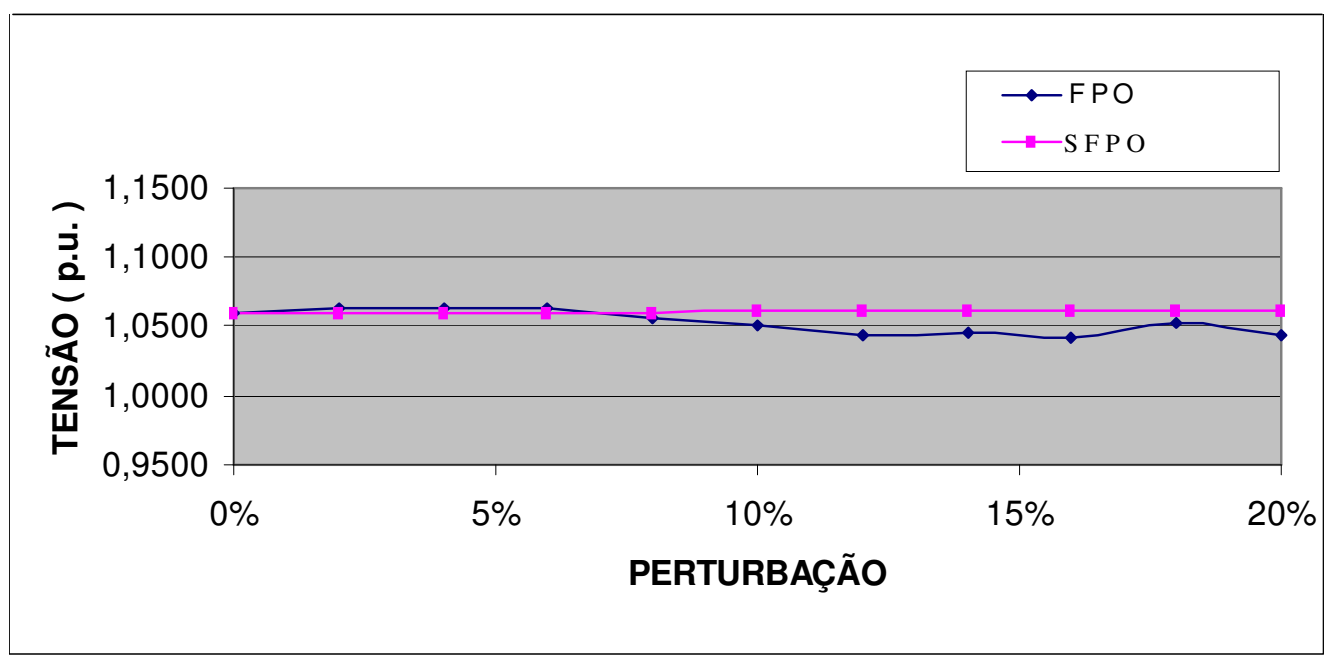

Figura 6.25 - Magnitude da tensão na barra 9

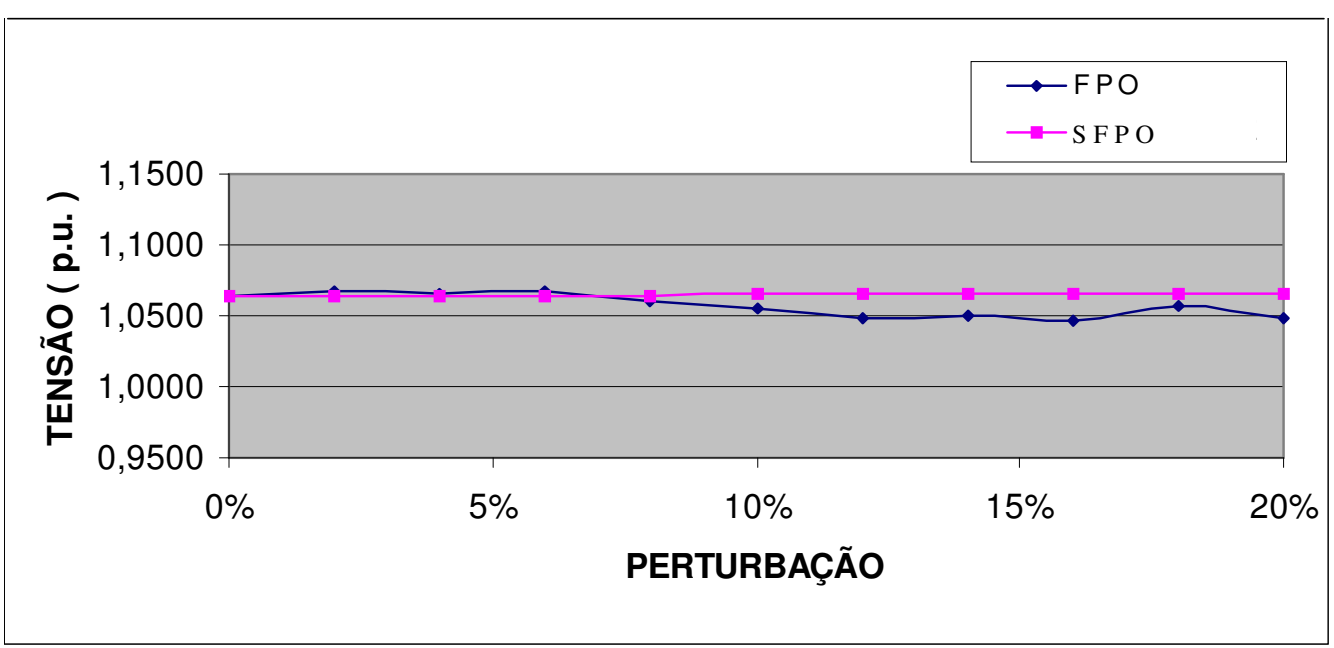

Figura 6.26 - Magnitude da tensão na barra 10

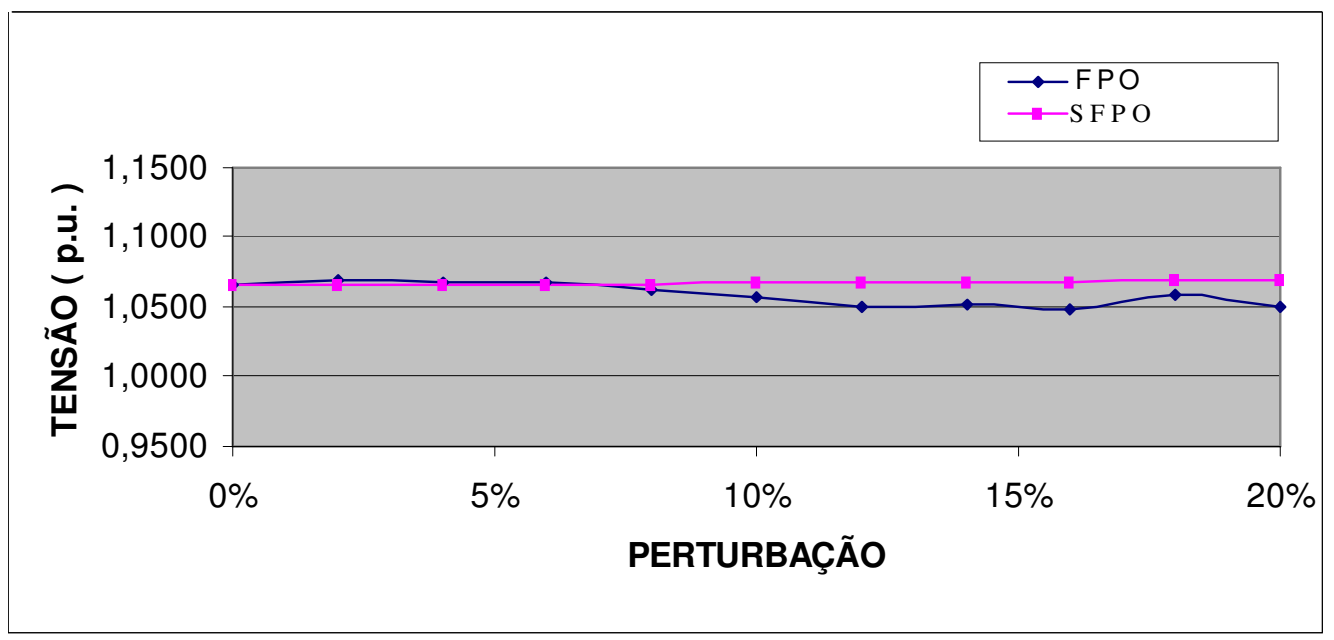

Figura 6.27 - Magnitude da tensão na barra 11 


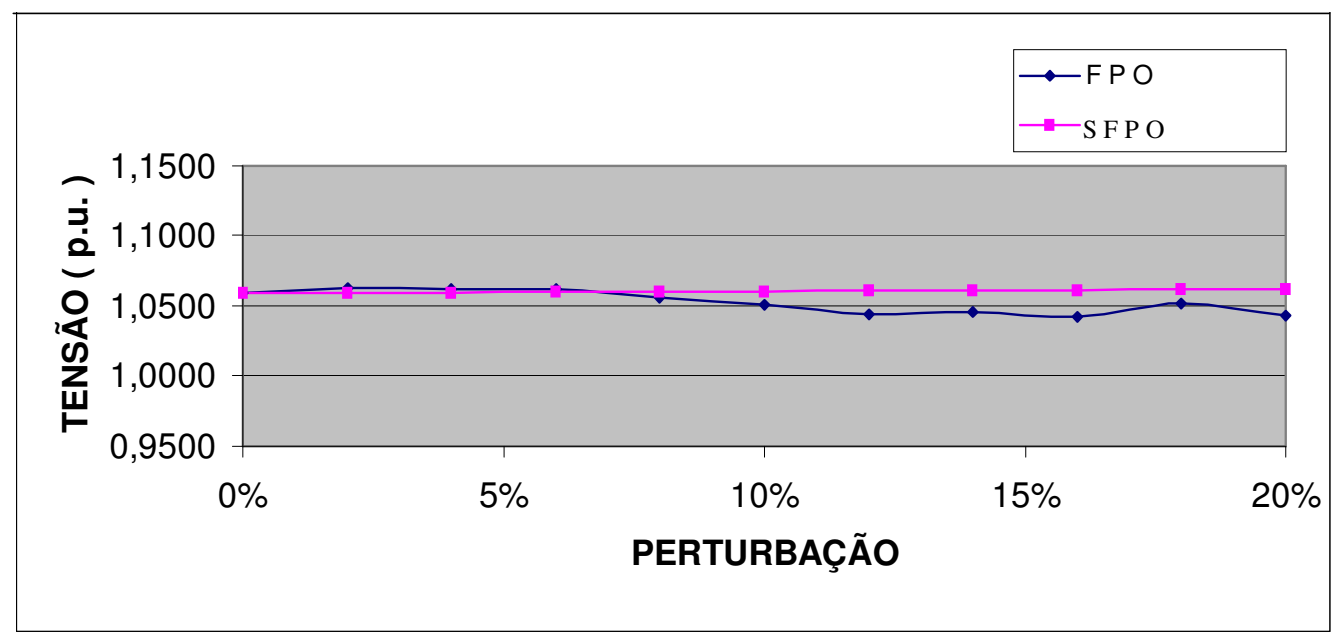

Figura 6.28 - Magnitude da tensão na barra 12

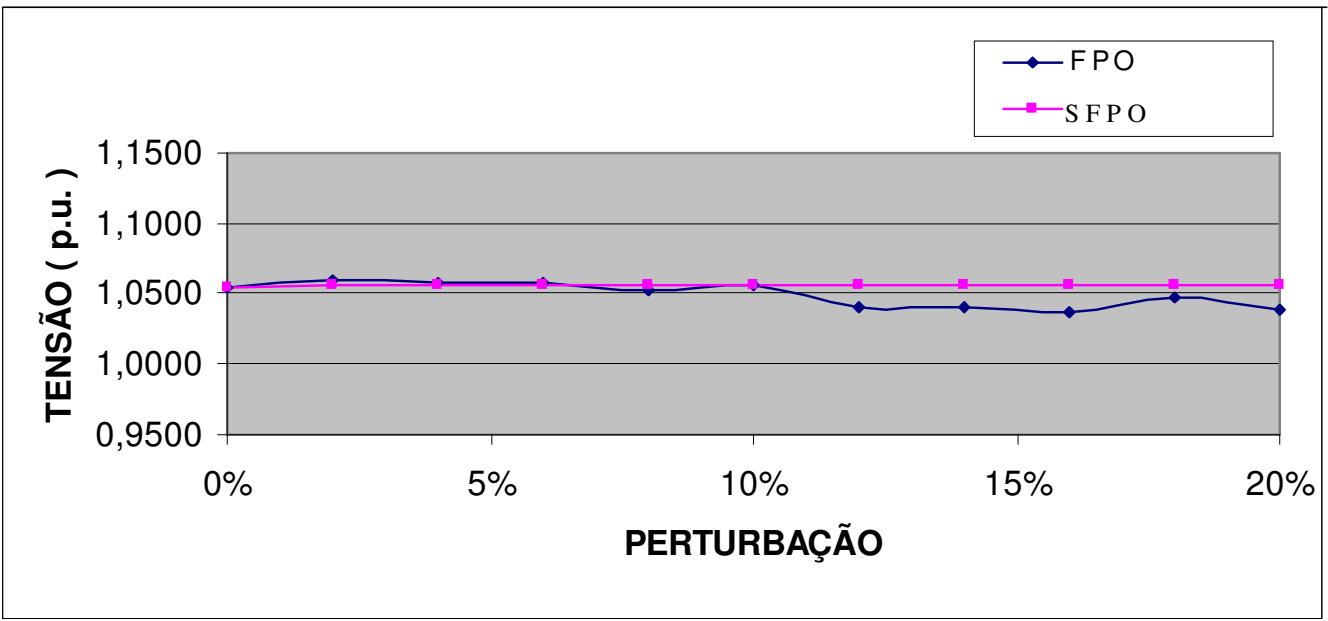

Figura 6.29 - Magnitude da tensão na barra 13

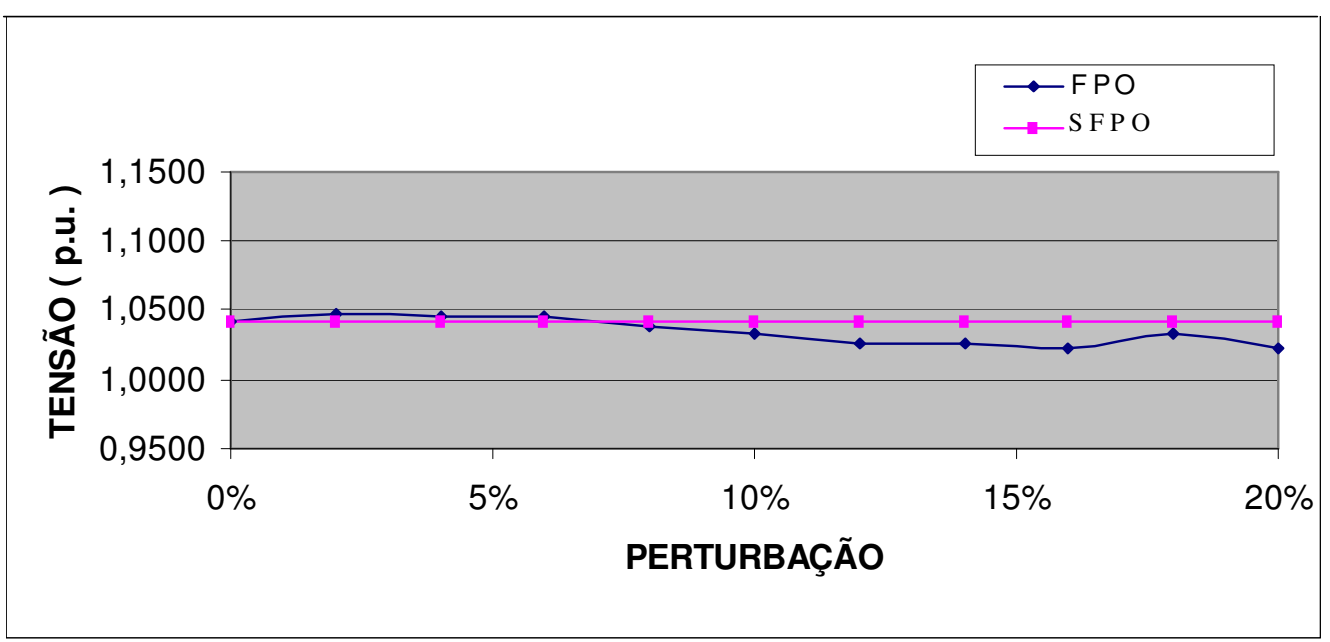

Figura 6.30 - Magnitude da tensão na barra 14 
Vimos pelo conjunto de Figuras anteriores, que relaciona a magnitude de tensão à perturbação no sistema, a variação da magnitude da tensão no sistema para os programas de FPO e SFPO. As magnitudes de tensão obtidas através do programa SFPO mantiveram-se muito próximas das obtidas pelo FPO para perturbações inferiores a 5\%. A tensão na barra slack permaneceu no seu limite superior $(1,10$ p.u.), pelo fato de estar ativa na solução ótima do caso inicial.

A seguir apresentaremos as curvas da geração de potência reativa das barras de geração.

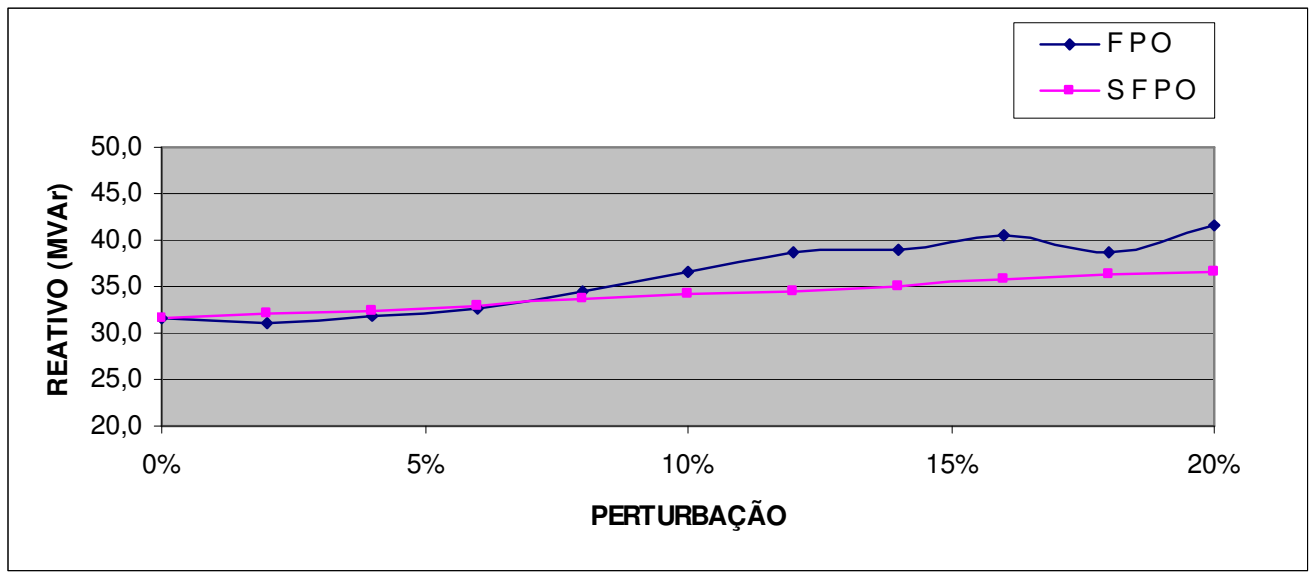

Figura 6.31 - Geração de potência reativa na barra 2 


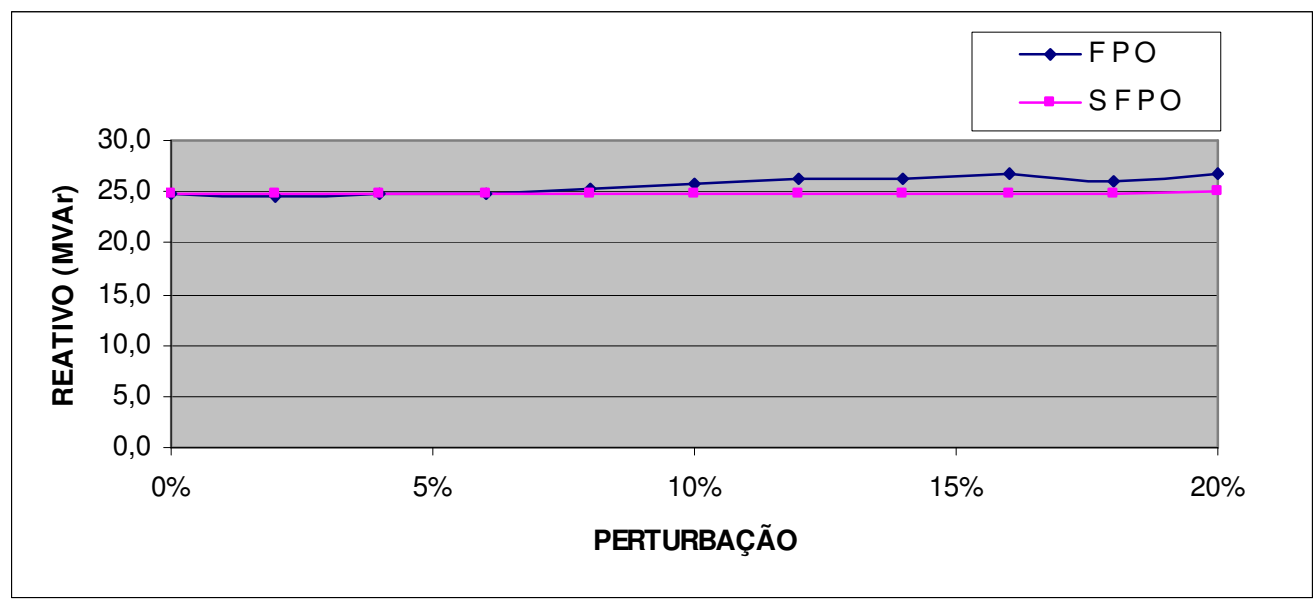

Figura 6.32 - Geração de potência reativa na barra 3

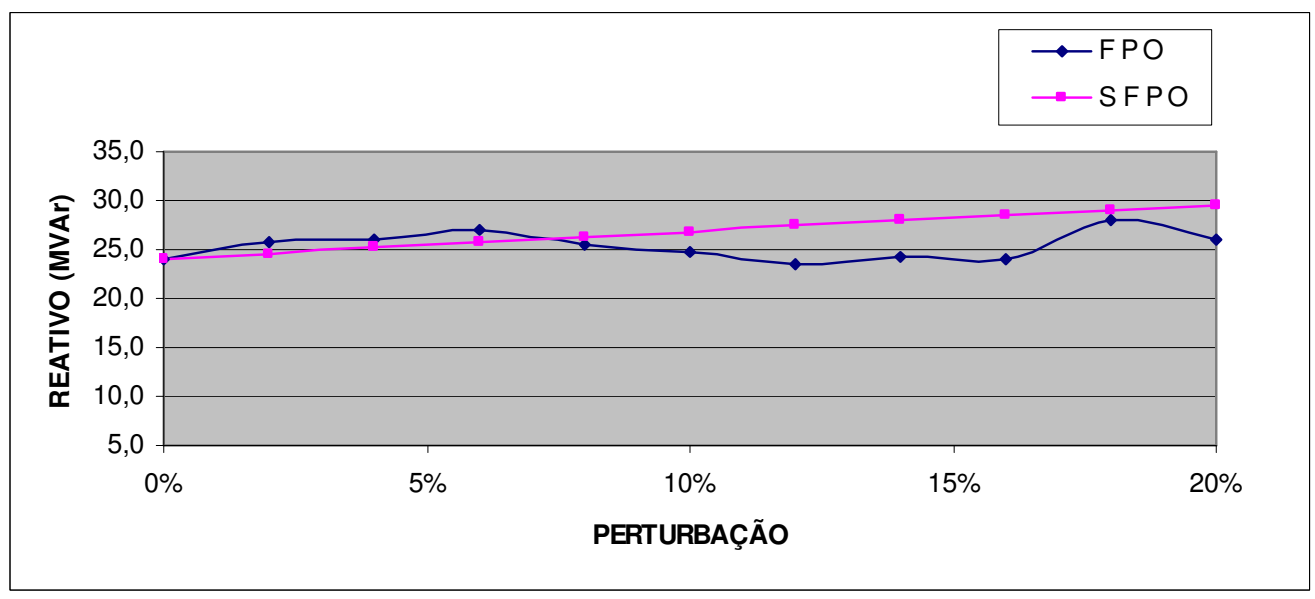

Figura 6.33 - Geração de potência reativa na barra 6

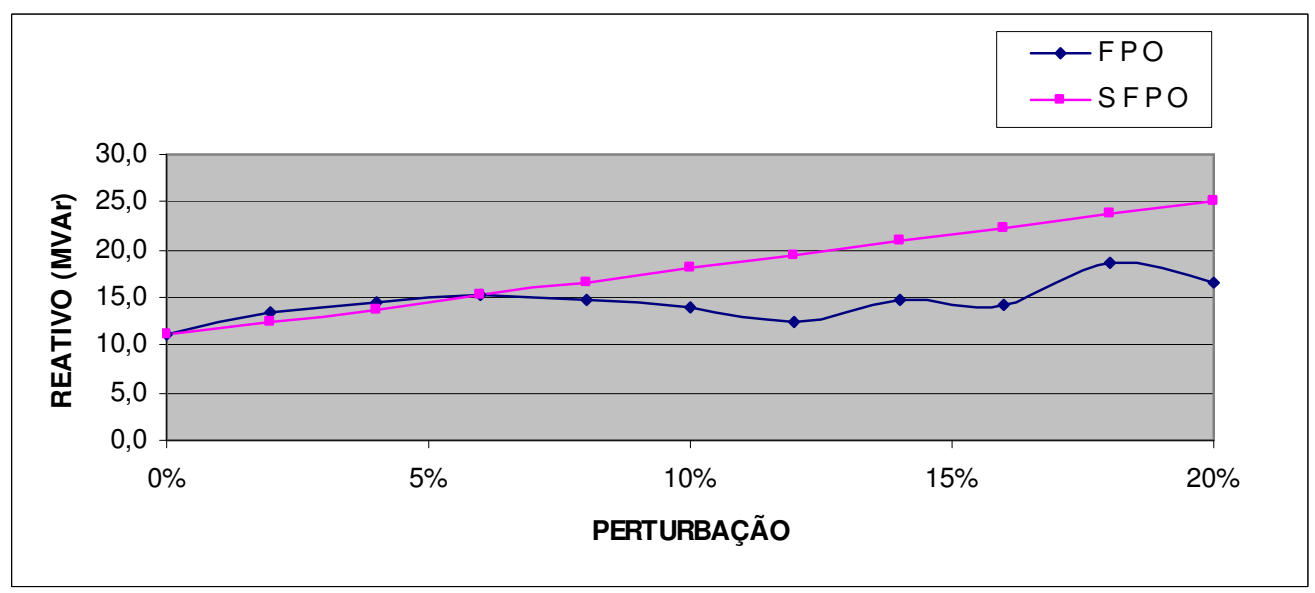

Figura 6.34 - Geração de potencia reativa na barra 8 
Vimos através do conjunto de Figuras anteriores, que relaciona a injeção de potência reativa com a perturbação no sistema, a variação da injeção de potência reativa no sistema. As gerações de potência reativa obtidas via SFPO e FPO mantiveram-se dentro dos limites pré-estabelecidos.

A Figura 6.35 mostra os maiores erros em p.u. das equações de balanço calculadas em relação à perturbação pelo programa de SFPO. O maior erro da potência ativa ocorreu na barra 4, e o maior erro da potência reativa ocorreu na barra 5. Para valores inferiores a 5\% de perturbação, o erro das equações de balanço manteve-se em valores muito pequenos, como pode ser observado na Figura 6.35.

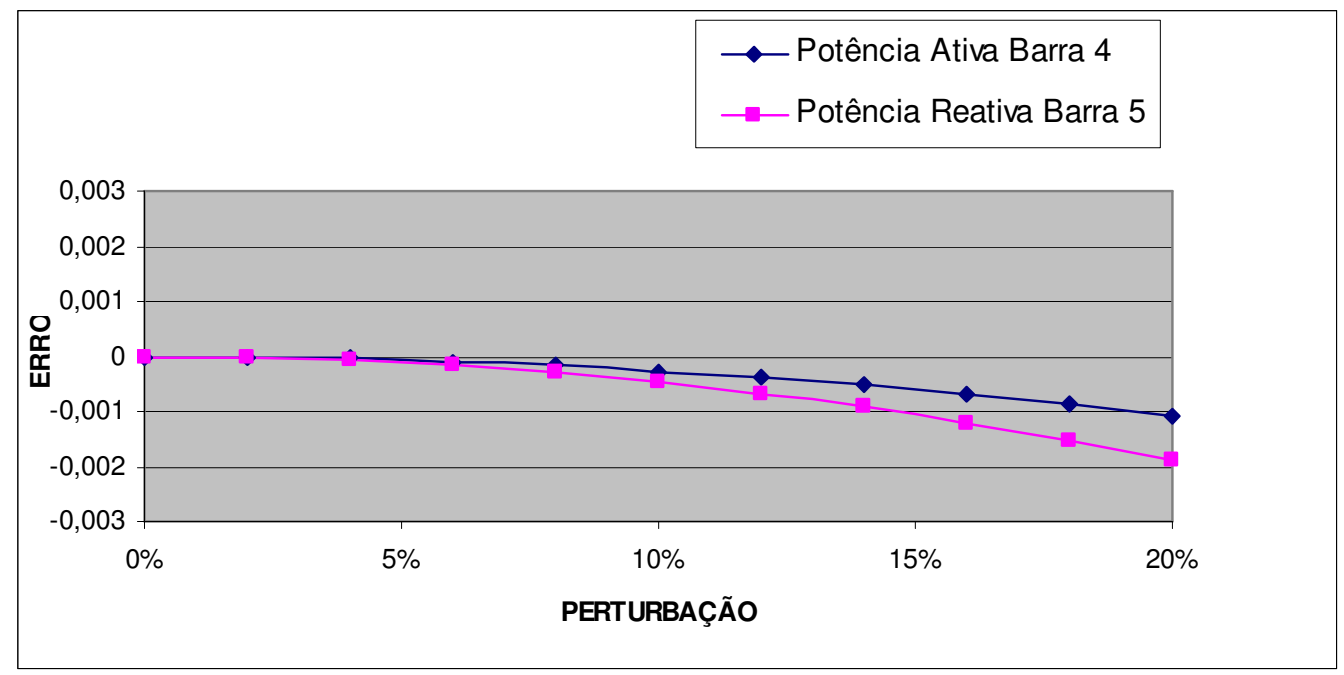

Figura 6.35 - Maior erro da potência ativa e reativa 


\subsection{EXEMPLO 3: SISTEMA 30 BARRAS}

No sistema IEEE 30 barras, representado pela Figura 6.36, fazemos um acréscimo de carga de 5\% em todas as barras de carga e utilizamos a SFPO para estimar a nova solução.

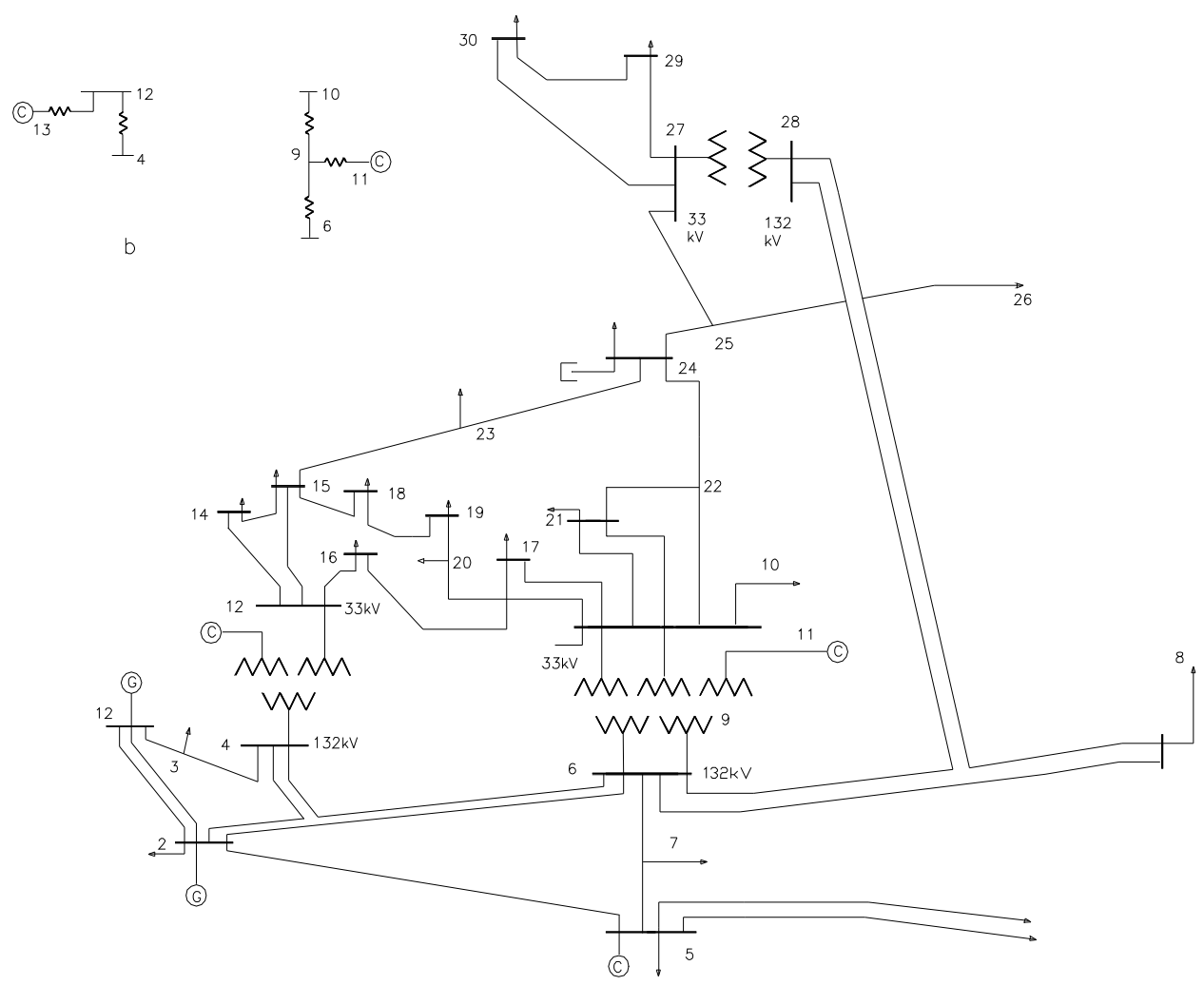

Figura 6.36 - Sistema IEEE 30 barras

A seguir apresentamos um resumo da solução do sistema antes e após a perturbação. A Tabela 6.8 apresenta a solução ótima do caso base $(\varepsilon=0,0)$ e a Tabela 6.9 apresenta a solução estimada pela SFPO após a perturbação no sistema. 
TABELA 6.8 - Solução ótima do sistema 30 barras $(\varepsilon=0,0)$ usada pela SFPO

\begin{tabular}{|c|c|c|}
\hline Barras k & $\mathrm{V}_{\mathrm{k}}$ (p.u.) & $\theta_{\mathrm{k}}$ (graus) \\
\hline 1 & 1,1000 & 0,0000 \\
\hline 2 & 1,0819 & $-4,9276$ \\
\hline 3 & 1,0639 & $-6,9875$ \\
\hline 4 & 1,0553 & $-8,6113$ \\
\hline 5 & 1,0469 & $-13,0530$ \\
\hline 6 & 1,0505 & $-10,2456$ \\
\hline 7 & 1,0416 & $-11,8931$ \\
\hline 8 & 1,0513 & $-10,9565$ \\
\hline 9 & 1,0530 & $-13,3326$ \\
\hline 10 & 1,0457 & $-14,9722$ \\
\hline 11 & 1,0716 & $-13,3326$ \\
\hline 12 & 1,0504 & $-14,2300$ \\
\hline 13 & 1,0744 & $-14,2300$ \\
\hline 14 & 1,0361 & $-15,0821$ \\
\hline 15 & 1,0328 & $-15,2079$ \\
\hline 16 & 1,0403 & $-14,8483$ \\
\hline 17 & 1,0388 & $-15,1422$ \\
\hline 18 & 1,0252 & $-15,8273$ \\
\hline 19 & 1,0238 & $-16,0198$ \\
\hline 20 & 1,0284 & $-15,8064$ \\
\hline 21 & 1,0332 & $-15,4058$ \\
\hline 22 & 1,0336 & $-15,3938$ \\
\hline 23 & 1,0236 & $-15,5693$ \\
\hline 24 & 1,0204 & $-15,7609$ \\
\hline 25 & 1,0193 & $-15,3809$ \\
\hline 26 & 1,0015 & $-15,9485$ \\
\hline 27 & 1,0271 & $-14,8096$ \\
\hline 28 & 1,0470 & $-10,8395$ \\
\hline 29 & 1,0071 & $-15,9880$ \\
\hline 30 & 0,9949 & $-16,9345$ \\
\hline Perdas & \multicolumn{2}{|c|}{$16,2792 \mathrm{MW}$} \\
\hline
\end{tabular}


TABELA 6.9 - Solução do sistema 30 barras estima pela SFPO após a perturbação

\begin{tabular}{|c|c|c|}
\hline Barras k & $\mathrm{V}_{\mathrm{k}}$ (p.u.) & $\theta_{\mathrm{k}}$ (graus) \\
\hline 1 & 1,1000 & 0,0000 \\
\hline 2 & 1,0802 & $-5,0554$ \\
\hline 3 & 1,0622 & $-7,21608$ \\
\hline 4 & 1,0533 & $-8,89574$ \\
\hline 5 & 1,0447 & $-13,3225$ \\
\hline 6 & 1,0484 & $-10,5744$ \\
\hline 7 & 1,0389 & $-12,224$ \\
\hline 8 & 1,0486 & $-11,285$ \\
\hline 9 & 1,0564 & $-13,8379$ \\
\hline 10 & 1,0461 & $-15,5671$ \\
\hline 11 & 1,0864 & $-13,8379$ \\
\hline 12 & 1,0498 & $-14,7858$ \\
\hline 13 & 1,0756 & $-14,7858$ \\
\hline 14 & 1,0349 & $-15,6799$ \\
\hline 15 & 1,0315 & $-15,8121$ \\
\hline 16 & 1,0397 & $-15,4387$ \\
\hline 17 & 1,0387 & $-15,7452$ \\
\hline 18 & 1,0239 & $-16,4646$ \\
\hline 19 & 1,0226 & $-16,6676$ \\
\hline 20 & 1,0276 & $-16,4433$ \\
\hline 21 & 1,0328 & $-16,0207$ \\
\hline 22 & 1,0331 & $-16,0075$ \\
\hline 23 & 1,0218 & $-16,1866$ \\
\hline 24 & 1,0185 & $-16,3805$ \\
\hline 25 & 1,0164 & $-15,9728$ \\
\hline 26 & 0,9977 & $-16,5725$ \\
\hline 27 & 1,0241 & $-15,3665$ \\
\hline 28 & 1,0446 & $-11,19$ \\
\hline 29 & 1,0030 & $-16,6119$ \\
\hline 30 & 0,9902 & $-17,6141$ \\
\hline Perdas & \multicolumn{2}{|c|}{$17,2223 \mathrm{MW}$} \\
\hline
\end{tabular}

As tensões e as injeções de potência reativa permaneceram dentro dos seus limites. Os maiores erros nas equações de balanço ocorreram nas barras 6 e 3, respectivamente para as potências ativas e reativas, os quais ficaram na ordem de $10^{-4}$ p.u. As perdas de potência ativa na transmissão passaram de 16,27 MW para 17,22 MW. A geração de potência ativa na barra slack teve um acréscimo de 7,8 MW após a perturbação. 


\subsection{EXEMPLO 4: SISTEMA CESP 53 BARRAS}

Para o sistema CESP 53 barras, representado pela Figura 6.37, fazemos um corte de carga de $6 \%$ nas barras de carga 35, 36 e 37, mantendo o fator de potência constante.

Figura 6.37 - Sistema CESP 53 barras

Utilizando a SFPO, estimamos a solução para o sistema perturbado e analisamos o resultado. A Tabela 6.10 apresenta a solução ótima do caso base $(\varepsilon=0,0)$, e a Tabela 6.11, a solução estimada pela SFPO após a perturbação no sistema. 
TABELA 6.10 - Solução ótima do sistema CESP $(\varepsilon=0,0)$ usada pela SFPO

\begin{tabular}{|c|c|c|}
\hline Barras k & $\mathrm{V}_{\mathrm{k}}$ (p.u.) & $\theta_{\mathrm{k}}$ (graus) \\
\hline 1 & 0,9905 & 0,0000 \\
\hline 2 & 0,9687 & $-1,3881$ \\
\hline 3 & 1,0746 & $-13,0503$ \\
\hline 4 & 1,0861 & $-14,6182$ \\
\hline 5 & 1,0596 & $-16,6806$ \\
\hline 6 & 1,0688 & $-9,9807$ \\
\hline 7 & 1,0240 & $-5,2114$ \\
\hline 8 & 0,9752 & 0,5037 \\
\hline 9 & 1,0236 & $-8,4046$ \\
\hline 10 & 1,0128 & $-4,4649$ \\
\hline 11 & 0,9964 & $-8,6452$ \\
\hline 12 & 1,0188 & $-5,3048$ \\
\hline 13 & 1,0641 & $-1,3575$ \\
\hline 14 & 1,0456 & 8,3837 \\
\hline 15 & 1,0361 & 3,1379 \\
\hline 16 & 1,0595 & 3,6067 \\
\hline 17 & 1,0648 & $-3,8655$ \\
\hline 18 & 1,0832 & 2,6224 \\
\hline 19 & 1,0679 & $-3,5483$ \\
\hline 20 & 1,0279 & $-8,5511$ \\
\hline 21 & 1,0616 & $-11,3063$ \\
\hline 22 & 1,0391 & $-13,5135$ \\
\hline 23 & 1,0692 & $-20,1781$ \\
\hline 24 & 1,0505 & $-31,1901$ \\
\hline 25 & 1,0213 & $-25,7056$ \\
\hline 26 & 1,0764 & $-23,1521$ \\
\hline 27 & 1,0613 & $-26,3471$ \\
\hline 28 & 1,0214 & $-27,8848$ \\
\hline 29 & 0,9663 & $-32,4981$ \\
\hline 30 & 0,9607 & $-37,2011$ \\
\hline 31 & 1,0238 & $-30,5146$ \\
\hline 32 & 0,9987 & $-38,4774$ \\
\hline 33 & 1,0284 & $-33,1115$ \\
\hline 34 & 1,0241 & $-30,9956$ \\
\hline 35 & 0,9618 & $-38,3903$ \\
\hline 36 & 0,9914 & $-36,5747$ \\
\hline 37 & 1,0553 & $-41,5598$ \\
\hline 38 & 1,0337 & $-40,8111$ \\
\hline 39 & 0,9585 & $-45,4227$ \\
\hline 40 & 1,0291 & $-43,8530$ \\
\hline 41 & 1,0544 & $-30,3967$ \\
\hline 42 & 1,0470 & $-29,9675$ \\
\hline 43 & 1,0752 & $-30,3967$ \\
\hline 44 & 1,0341 & $-33,8979$ \\
\hline 45 & 1,0653 & $-29,7145$ \\
\hline 46 & 1,0393 & $-34,9076$ \\
\hline 47 & 1,0299 & $-32,4524$ \\
\hline 48 & 1,0215 & $-34,5442$ \\
\hline 49 & 0,9954 & $-37,9102$ \\
\hline 50 & 1,0468 & $-31,0128$ \\
\hline 51 & 1,0388 & $-30,9597$ \\
\hline 52 & 1,0332 & $-34,7677$ \\
\hline 53 & 1,0750 & $-31,0128$ \\
\hline Perdas & \multicolumn{2}{|c|}{$213,4517 \mathrm{MW}$} \\
\hline
\end{tabular}


TABELA 6.10 - Solução do sistema CESP estima pela SFPO após a perturbação

\begin{tabular}{|c|c|c|}
\hline Barras k & $\mathrm{V}_{\mathrm{k}}$ (p.u.) & $\theta_{\mathrm{k}}$ (graus) \\
\hline 1 & 0,9884 & 0,0000 \\
\hline 2 & 0,9666 & $-1,2220$ \\
\hline 3 & 1,0731 & $-12,9171$ \\
\hline 4 & 1,0846 & $-14,4894$ \\
\hline 5 & 1,0581 & $-16,5578$ \\
\hline 6 & 1,0671 & $-9,8383$ \\
\hline 7 & 1,0222 & $-5,1500$ \\
\hline 8 & 0,9732 & 0,6343 \\
\hline 9 & 1,0216 & $-8,2670$ \\
\hline 10 & 1,0108 & $-4,3541$ \\
\hline 11 & 0,9944 & $-8,5506$ \\
\hline 12 & 1,0168 & $-5,1550$ \\
\hline 13 & 1,0614 & $-1,1139$ \\
\hline 14 & 1,0427 & 8,6797 \\
\hline 15 & 1,0329 & 3,4069 \\
\hline 16 & 1,0566 & 3,8766 \\
\hline 17 & 1,0621 & $-3,6348$ \\
\hline 18 & 1,0800 & 2,9007 \\
\hline 19 & 1,0653 & $-3,3039$ \\
\hline 20 & 1,0251 & $-8,3330$ \\
\hline 21 & 1,0595 & $-11,0808$ \\
\hline 22 & 1,0371 & $-13,2967$ \\
\hline 23 & 1,0678 & $-19,9938$ \\
\hline 24 & 1,0491 & $-30,9809$ \\
\hline 25 & 1,0198 & $-25,5366$ \\
\hline 26 & 1,0754 & $-22,9660$ \\
\hline 27 & 1,0602 & $-26,1674$ \\
\hline 28 & 1,0207 & $-27,6573$ \\
\hline 29 & 0,9656 & $-32,2768$ \\
\hline 30 & 0,9602 & $-36,9137$ \\
\hline 31 & 1,0235 & $-30,2219$ \\
\hline 32 & 0,9976 & $-38,2005$ \\
\hline 33 & 1,0274 & $-32,8237$ \\
\hline 34 & 1,0238 & $-30,6783$ \\
\hline 35 & 0,9653 & $-37,7708$ \\
\hline 36 & 0,9935 & $-35,9116$ \\
\hline 37 & 1,0502 & $-41,1403$ \\
\hline 38 & 1,0297 & $-40,4300$ \\
\hline 39 & 0,9542 & $-45,0800$ \\
\hline 40 & 1,0251 & $-43,4952$ \\
\hline 41 & 1,0531 & $-30,1609$ \\
\hline 42 & 1,0457 & $-29,7306$ \\
\hline 43 & 1,0732 & $-30,1609$ \\
\hline 44 & 1,0327 & $-33,6712$ \\
\hline 45 & 1,0641 & $-29,5112$ \\
\hline 46 & 1,0380 & $-34,7168$ \\
\hline 47 & 1,0288 & $-32,1944$ \\
\hline 48 & 1,0203 & $-34,2908$ \\
\hline 49 & 0,9942 & $-37,6645$ \\
\hline 50 & 1,0454 & $-30,7831$ \\
\hline 51 & 1,0374 & $-30,7299$ \\
\hline 52 & 1,0318 & $-34,5481$ \\
\hline 53 & 1,0727 & $-30,7831$ \\
\hline Perdas & \multicolumn{2}{|c|}{$210,9682 \mathrm{MW}$} \\
\hline
\end{tabular}


Após a perturbação, todas as tensões e as injeções de potência reativa permaneceram dentro dos seus limites. Os maiores erros nas equações de balanço ocorreram nas barras 34 e 36 para as potências ativas e reativas respectivamente, e foram menores que $9 \times 10^{-4}$ p.u. As perdas no sistema passaram de 213,45 MW para 210,96 MW. A geração da barra slack, representada no sistema pela usina de Ilha Solteira, teve uma redução de 41,2 MW na sua geração após a perturbação.

\subsection{EXEMPLO 4: SISTEMA IEEE 118 BARRAS}

No sistema IEEE 118 barras, representado pela Figura 6.38, simulamos um corte de carga de $100 \%$ na barra de carga 102 e analisamos a solução obtida pela SFPO. No Apêndice B, encontram-se as Tabelas com os dados das variáveis primais para o ponto ótimo inicial e para o ponto estimado após a perturbação.

Após a perturbação, todas as tensões e as injeções de potência reativa permaneceram dentro dos seus limites - com exceção da injeção de potência reativa da barra 77 que excedeu 0,3 MVAr do seu limite superior. O maiores erros para potência ativa e reativa ocorreram na barra de carga 102 e foram menores que $3 \times 10^{-4}$ p.u. As perdas no sistema passaram de 131,56 MW para 131,55 MW após o corte de carga, e a barra slack teve uma redução de 5,1 MW na sua geração após a perturbação. 


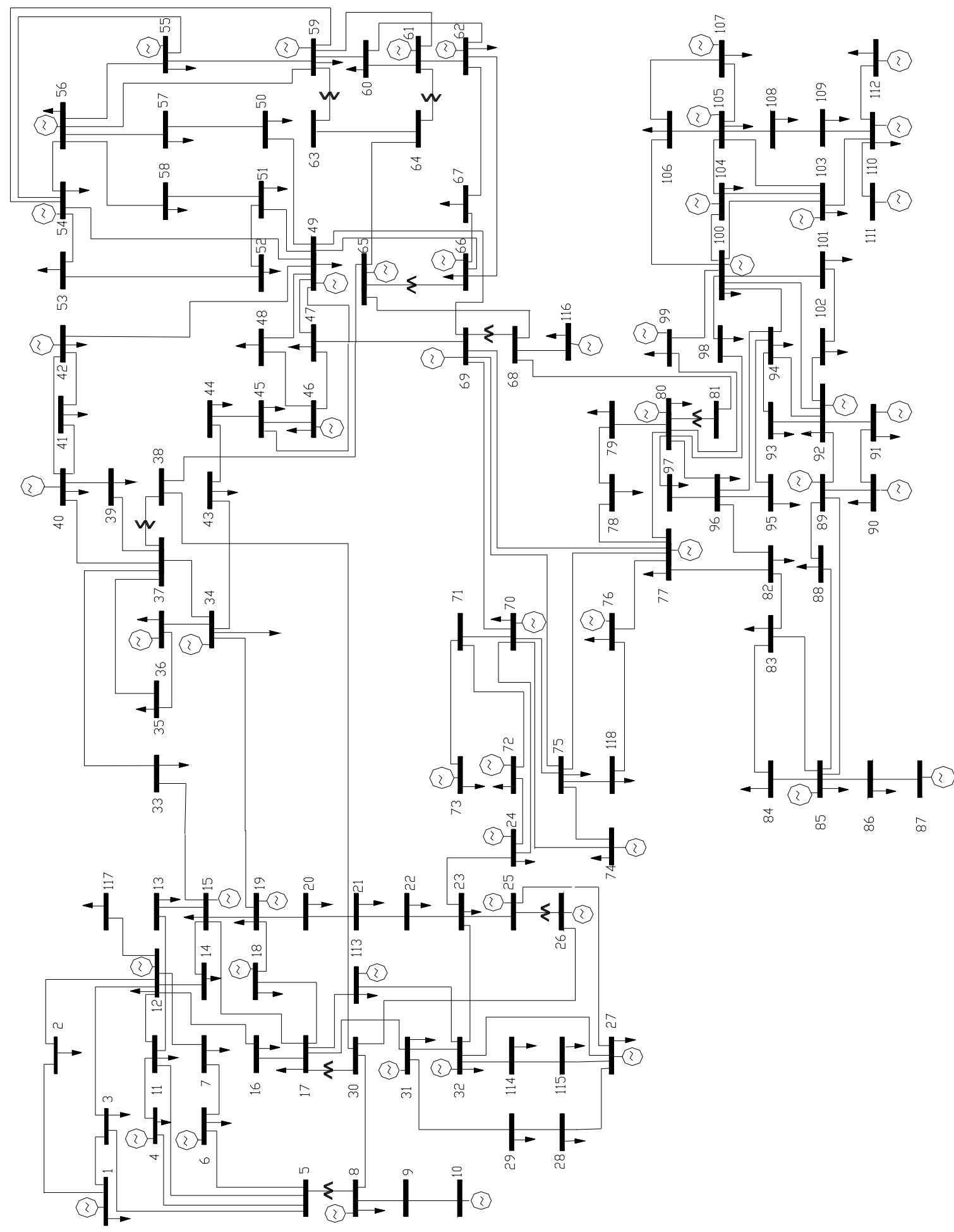

Figura 6.38 - Sistema IEEE 118 barras 


\subsection{EXEMPLO 5: SISTEMA 162 BARRAS}

Para o sistema IEEE 162 barras, simulamos um acréscimo de carga e analisamos a solução obtida pela SFPO. O acréscimo de carga foi de $1 \%$ em todas as barras de carga do sistema, mantendo o fator de potência constante. Utilizamos a SFPO para estimar a nova solução. No Apêndice B encontram-se as Tabelas com os dados das variáveis primais para o ponto ótimo inicial e para o ponto estimado após a perturbação.

Após a perturbação, as tensões e as injeções de potência reativa permaneceram dentro dos seus limites. Os maiores erros nas equações de balanço ocorreram na barra 93 e foram menores que $10^{-3}$ p.u. para a potência ativa, e menor que $3 \times 10^{-3}$ p.u para potência reativa. As perdas no sistema passaram de 158,30 MW para 161,04 MW após o acréscimo de carga. A barra slack teve um acréscimo de 55,0 MW em sua geração após a perturbação.

\subsection{EXEMPLO 6: SISTEMA IEEE 300 BARRAS}

No sistema IEEE 300 barras, realizamos um acréscimo de carga de 5\% nas barras de carga 49, 51, 52 e 55, mantendo o fator de potência constante. Utilizamos a SFPO para estimar a solução após a perturbação. Nesse teste analisamos os resultados e a relação de sensibilidade deste acréscimo de carga em relação à injeção de potência reativa nas barras de geração. No Apêndice B, encontram-se as Tabelas com os dados das variáveis primais para o ponto ótimo inicial e para o ponto estimado pela SFPO após a perturbação. 
Depois de realizada a perturbação, as tensões e as injeções de potência reativa permaneceram dentro dos seus limites. Os maiores erros nas equações de balanço ocorreram na barra de carga 526. O erro para potência ativa foi menor que $9 \times 10^{-4}$ p.u., e o erro para potência reativa, menor que $2 \times 10^{-4}$ p.u. As perdas no sistema passaram de 408,31 MW para 410,06 MW após o acréscimo de carga. A barra slack teve um acréscimo de 9,7 MW em sua geração após a perturbação.

A Tabela 6.11 mostra a variação da injeção de potência reativa com o aumento de carga no sistema. A maior variação de injeção de potência reativa em MVAr ocorreu na barra 7049 (slack) e foi de 13,0 MVAr e a maior variação em porcentagem de MVAr ocorreu na barra 7024 e foi de 350,00\%.

TABELA 6.11 - Análise de sensibilidade da injeção de potência reativa no sistema em relação ao aumento da carga

\begin{tabular}{|c|c|c|c|c|}
\hline Barra k & $\begin{array}{l}\text { MVAr no Ponto } \\
\text { ótimo }(\varepsilon=0)\end{array}$ & $\begin{array}{c}\text { MVAr Após a } \\
\text { Perturbação }\end{array}$ & $\begin{array}{c}\text { Variação em } \\
\text { módulo de } \\
\text { MVAr }\end{array}$ & $\begin{array}{c}\text { Variação em \% } \\
\text { de MVAr }\end{array}$ \\
\hline 7049 & $-72,8$ & $-85,8$ & 13,0 & 17,86 \\
\hline 8 & $-59,9$ & $-59,2$ & 0,7 & 1,17 \\
\hline 10 & 56,6 & 57,7 & 1,1 & 1,94 \\
\hline 20 & 102,9 & 101,6 & 1,3 & 1,26 \\
\hline 63 & 52,0 & 49,0 & 3 & 5,77 \\
\hline 76 & 143,3 & 143,7 & 0,4 & 0,28 \\
\hline 84 & 120,1 & 120,2 & 0,1 & 0,08 \\
\hline 91 & 81,5 & 81,0 & 0,5 & 0,61 \\
\hline 92 & 81,8 & 81,6 & 0,2 & 0,24 \\
\hline 98 & $-60,4$ & $-59,8$ & 0,6 & 0,99 \\
\hline 108 & 3,2 & 3,9 & 0,7 & 21,88 \\
\hline 119 & 981,6 & 987,5 & 5,9 & 0,60 \\
\hline 124 & 98,2 & 97,7 & 0,5 & 0,51 \\
\hline 125 & 191,5 & 191,0 & 0,5 & 0,26 \\
\hline 138 & 192,4 & 192,5 & 0,1 & 0,05 \\
\hline 141 & 72,3 & 72,4 & 0,1 & 0,14 \\
\hline 143 & 267,9 & 276,1 & 8,2 & 3,06 \\
\hline 146 & 17,7 & 18,2 & 0,5 & 2,82 \\
\hline 147 & $-44,5$ & $-44,2$ & 0,3 & 0,67 \\
\hline 149 & 24,0 & 24,1 & 0,1 & 0,42 \\
\hline 152 & $-48,9$ & $-48,8$ & 0,1 & 0,20 \\
\hline 153 & $-15,1$ & $-14,9$ & 0,2 & 1,32 \\
\hline 156 & $-6,2$ & $-5,9$ & 0,3 & 4,84 \\
\hline
\end{tabular}




\begin{tabular}{|c|c|c|c|c|}
\hline 170 & 78,2 & 79,4 & 1,2 & 1,53 \\
\hline 171 & 447,6 & 448,5 & 0,9 & 0,20 \\
\hline 176 & 34,1 & 34,6 & 0,5 & 1,47 \\
\hline 177 & 39,9 & 40,8 & 0,9 & 2,26 \\
\hline 185 & 19,1 & 18,9 & 0,2 & 1,05 \\
\hline 186 & 274,6 & 272,3 & 2,3 & 0,84 \\
\hline 187 & 215,9 & 214,3 & 1,6 & 0,74 \\
\hline 190 & 145,1 & 143,8 & 1,3 & 0,90 \\
\hline 191 & 696 & 698,7 & 2,7 & 0,39 \\
\hline 198 & 66,2 & 66,2 & 0,0 & 0,00 \\
\hline 213 & $-10,1$ & $-9,7$ & 0,4 & 3,96 \\
\hline 220 & $-3,9$ & $-6,0$ & 2,1 & 53,85 \\
\hline 221 & 22,1 & 27,7 & 5,6 & 25,34 \\
\hline 222 & 154,7 & 155,3 & 0,6 & 0,39 \\
\hline 227 & 248,1 & 250,3 & 2,2 & 0,89 \\
\hline 230 & 42,4 & 41,4 & 1,0 & 2,36 \\
\hline 233 & 116,4 & 118,2 & 1,8 & 1,55 \\
\hline 236 & 98,6 & 98,3 & 0,3 & 0,30 \\
\hline 238 & 374,1 & 373,4 & 0,7 & 0,19 \\
\hline 239 & 18,0 & 17,8 & 0,2 & 1,11 \\
\hline 241 & $-71,2$ & $-69,4$ & 1,8 & 2,53 \\
\hline 242 & 81,4 & 82,0 & 0,6 & 0,74 \\
\hline 243 & 74,3 & 73,4 & 0,9 & 1,21 \\
\hline 7001 & 229,4 & 225,5 & 3,9 & 1,70 \\
\hline 7002 & 139,9 & 137,1 & 2,8 & 2,00 \\
\hline 7003 & $-62,8$ & $-56,4$ & 6,4 & 10,19 \\
\hline 7011 & 67,4 & 66,4 & 1 & 1,48 \\
\hline 7012 & 92,7 & 92,5 & 0,2 & 0,22 \\
\hline 7017 & 438,3 & 437,1 & 1,2 & 0,27 \\
\hline 7023 & 94,1 & 91,6 & 2,5 & 2,66 \\
\hline 7024 & $-0,6$ & 1,5 & 2,1 & 350,00 \\
\hline 7039 & 164,8 & 168,6 & 3,8 & 2,31 \\
\hline 7044 & 7,1 & 11,6 & 4,5 & 63,38 \\
\hline 7055 & 6,8 & 6,9 & 0,1 & 1,47 \\
\hline 7057 & 75,6 & 76,5 & 0,9 & 1,19 \\
\hline 7061 & 191,6 & 197,3 & 5,7 & 2,97 \\
\hline 7062 & $-41,7$ & $-50,8$ & 9,1 & 21,82 \\
\hline 7071 & 45,6 & 46,0 & 0,4 & 0,88 \\
\hline 7130 & 501,6 & 502,5 & 0,9 & 0,18 \\
\hline 7139 & 147,4 & 149,1 & 1,7 & 1,15 \\
\hline 7166 & 111,4 & 113,3 & 1,9 & 1,71 \\
\hline 9002 & 0,9 & 1,0 & 0,1 & 11,11 \\
\hline 9051 & 15,7 & 15,7 & 0 & 0,00 \\
\hline 9053 & 8,5 & 8,5 & 0 & 0,00 \\
\hline 9054 & 39,4 & 41,1 & 1,7 & 4,31 \\
\hline 9055 & 0,2 & 0,7 & 0,5 & 250,00 \\
\hline
\end{tabular}




\subsection{EXEMPLO 7: SISTEMA 9 BARRAS}

Nesse exemplo testamos a metodologia no sistema de 9 barras, representado pela Figura 6.39. Para encontrar a solução ótima do sistema utilizamos o programa de Despacho Ótimo de Potência Ativa (DOPA) ALTIMARI (1999).

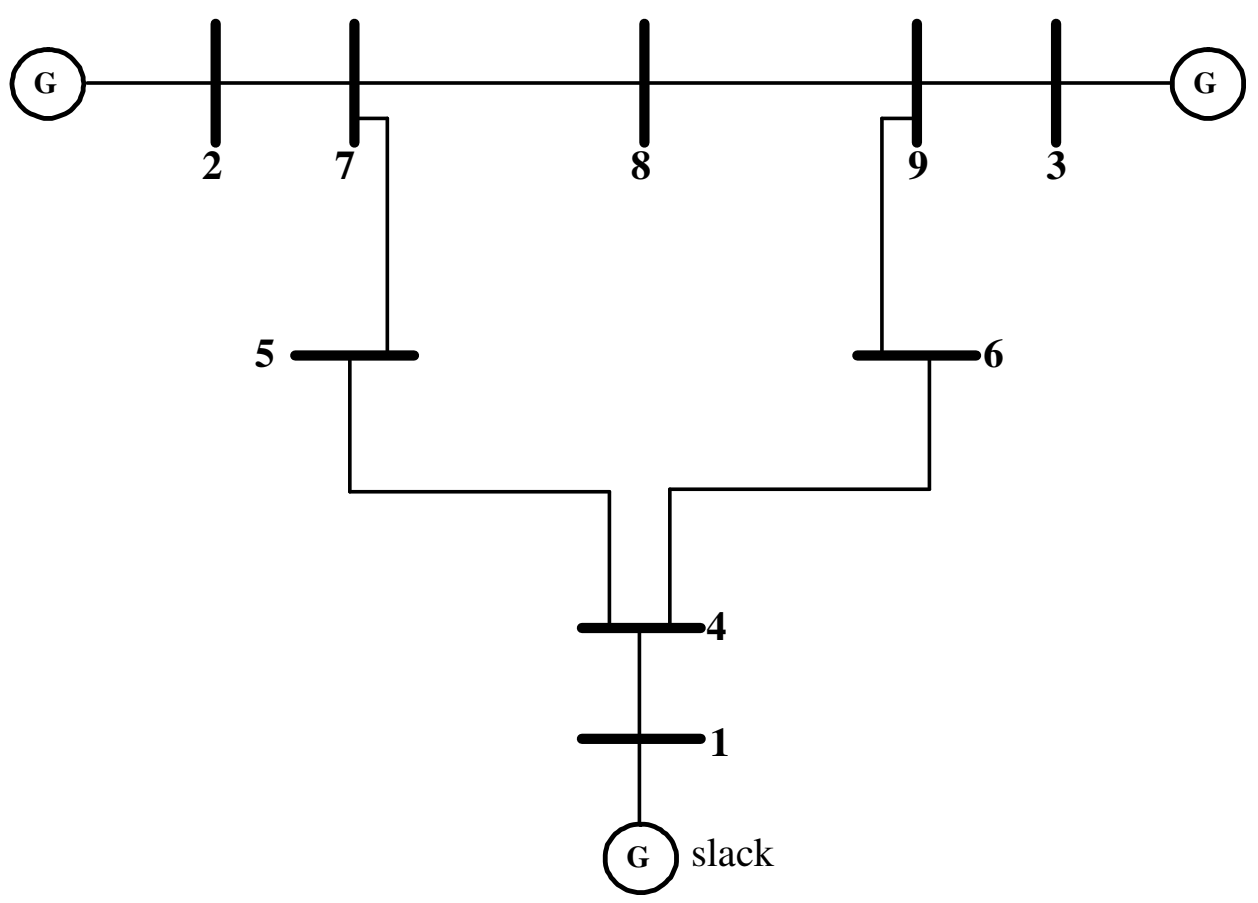

Figura 6.39 - Sistema de 9 barras

O objetivo do teste é comprovar a eficiência da metodologia SFPO também em problema de DOPA. Realizamos um aumento de $10 \%$ na barra de carga 8 . na Tabela 6.12 está apresentado um resumo da solução ótima do sistema antes da perturbação, e na Tabela 6.13, um resumo da solução obtida via SFPO após a perturbação. 
TABELA 6.12 - Solução ótima do sistema de 9 barras $(\varepsilon=0,0)$ usada pela SFPO

\begin{tabular}{|c|c|c|}
\hline Barra k & $\mathrm{V}_{\mathrm{k}}$ (p.u.) & $\theta_{\mathrm{k}}$ (graus) \\
\hline \hline 1 & 1,1000 & 0,0000 \\
\hline 2 & 1,0975 & $-2,9407$ \\
\hline 3 & 1,0876 & $-3,1820$ \\
\hline 4 & 1,0968 & $-4,3165$ \\
\hline 5 & 1,0756 & $-8,0311$ \\
\hline 6 & 1,0873 & $-7,2038$ \\
\hline 7 & 1,1000 & $-5,5546$ \\
\hline 8 & 1,0895 & $-7,3787$ \\
\hline 9 & 1,1000 & $-5,1908$ \\
\hline Perdas & \multicolumn{2}{|c|}{$2,3156 \mathrm{MW}$} \\
\hline
\end{tabular}

TABELA 6.13 - Solução do sistema de 9 barras estimada pela SFPO após a perturbação

\begin{tabular}{|c|c|c|}
\hline Barra $\mathrm{k}$ & $\mathrm{V}_{\mathrm{k}}(\mathrm{p} . \mathrm{u})$ & $\theta_{\mathrm{k}}$ (graus) \\
\hline \hline 1 & 1,1000 & 0,0000 \\
\hline 3 & 1,0991 & $-2,7692$ \\
\hline 4 & 1,0886 & $-3,0663$ \\
\hline 5 & 1,0968 & $-4,3165$ \\
\hline 6 & 1,0756 & $-8,0310$ \\
\hline 7 & 1,0873 & $-7,2038$ \\
\hline 8 & 1,1000 & $-5,5544$ \\
\hline 9 & 1,0875 & $-7,5747$ \\
\hline Perdas & 1,1000 & $-5,1910$ \\
\hline \hline
\end{tabular}

O teste mostrou que as tensões nas barras 1,7 e 9 continuaram nos seus limites superiores após a perturbação. As perdas no sistema passaram de 2,32 MW para 2,41 MW. O maiores erros para potência ativa e reativa ocorreram na barra de carga 8 e foram menores que $3 \times 10^{-4}$ p.u. As barras de gerações 2 e 3 foram as responsáveis por suprir o aumento de carga da barra 8, sendo que a barra slack não teve nenhuma contribuição devido a sua localização no sistema.

Com a finalidade de validar a aplicação da metodologia SFPO ao problema DOPA, apresentamos na Tabela 6.14 e 6.15 uma comparação dos resultados obtidos via DOPA e SFPO para a perturbação de $10 \%$ na barra de carga 8 . 
TABELA 6.14 - Comparação das variáveis primais, obtidas via DOPA e via SFPO para o problema perturbado

\begin{tabular}{|c||c|c||c|c||}
\hline \multicolumn{1}{|c||}{} & \multicolumn{2}{c||}{ DOPA } & \multicolumn{2}{c|}{ SFPO } \\
\hline \hline $\mathrm{k}$ & $\mathrm{V}_{\mathrm{k}}$ (p.u.) & $\theta_{\mathrm{k}}$ (graus) & $\mathrm{V}_{\mathrm{k}}$ (p.u.) & $\theta_{\mathrm{k}}$ (graus) \\
\hline \hline 1 & 1.100 & 0.00 & 1.100 & 0.00 \\
\hline 2 & 1.099 & -2.77 & 1.099 & -2.76 \\
\hline 3 & 1.088 & -3.07 & 1.088 & -3.06 \\
\hline 4 & 1.096 & -4.32 & 1.096 & -4.31 \\
\hline 5 & 1.075 & -8.03 & 1.075 & -8.03 \\
\hline 6 & 1.087 & -7.20 & 1.087 & -7.20 \\
\hline 7 & 1.100 & -5.55 & 1.100 & -5.55 \\
\hline 8 & 1.087 & -7.57 & 1.087 & -7.57 \\
\hline 9 & 1.100 & -5.19 & 1.100 & 5.19 \\
\hline
\end{tabular}

TABELA 6.15 - Comparação dos fluxos de potência ativa e reativa nas linhas, obtidos via SFPO e via SFPO para o problema perturbado

\begin{tabular}{|c||c|c||c|c||}
\hline \multicolumn{1}{|||}{} & \multicolumn{2}{c||}{ DOPA } & \multicolumn{2}{c||}{ SFPO } \\
\hline \hline $\mathrm{k}-\mathrm{m}$ & $\mathrm{P}_{\mathrm{km}}(\mathrm{MW})$ & $\mathrm{Q}_{\mathrm{km}}(\mathrm{MVAr})$ & $\mathrm{P}_{\mathrm{km}}(\mathrm{MW})$ & $\mathrm{Q}_{\mathrm{km}}(\mathrm{MVAr})$ \\
\hline \hline $1-4$ & 157,65 & 12,09 & 157,60 & 12,19 \\
\hline $2-7$ & 94,00 & 0,70 & 94,02 & 0,71 \\
\hline $3-9$ & 75,77 & $-19,74$ & 75,79 & $-19,65$ \\
\hline $4-5$ & 92,20 & 8,84 & 92,18 & 8,90 \\
\hline $4-6$ & 65,45 & $-8,65$ & 65,43 & $-8,61$ \\
\hline $5-7$ & $-33,54$ & $-26,67$ & $-33,56$ & $-26,62$ \\
\hline $6-9$ & $-25,15$ & $-23,08$ & $-25,18$ & $-23,06$ \\
\hline $7-8$ & 60,11 & 4,01 & 60,12 & 4,01 \\
\hline $8-9$ & $-50,13$ & $-18,89$ & $-50,15$ & $-18,93$ \\
\hline
\end{tabular}

Os resultados apresentados na Tabela 6.14 e 6.15 comprovam a eficiência da SFPO para problemas de DOPA. O teste mostrou que a metodologia SFPO responde muito bem nos problemas de DOPA, pois nesses problemas todas as barras de geração têm a função de contribuírem para suprir a variação na demanda, ao contrário do problema de FPO em que somente a barra slack tem essa função. O DOPA é utilizado como ferramenta para o Controlador Automático de Geração (CAG).

Neste capítulo realizamos várias aplicações da SFPO. Os resultados obtidos nos exemplos mostraram que a SFPO pode ser empregada na estimativa de novas 
soluções após a ocorrência de pequenas perturbações na demanda do sistema. A SFPO também pode ser utilizada na análise de sensibilidade de uma variável elétrica em relação a outra variável e também pode ser empregada em problemas de DOPA. 


\section{Capítulo 7}

\section{CONCLUSÕES}

Neste trabalho propusemos uma metodologia para resolução do problema de Fluxo de Potência Ótimo perturbado.

Inicialmente, apresentamos um histórico dos métodos de FPO e de sensibilidade, a fim de posicionar o trabalho cronologicamente e de obter suporte teórico.

Implementamos um FPO que apresenta a Função Lagrangiana AumentadaBarreira Logarítima, na qual as restrições de igualdade são tratadas pelo método de Newton, o subconjunto das restrições canalizadas, pelo método de barreira, e as restrições de desigualdade, pelo método da Função Lagrangiana Aumentada.

A solução do FPO nos dá o ponto ótimo para o caso base. Utilizando sensibilidade, desenvolvemos uma nova abordagem, SFPO, para estimar novas soluções depois de ocorridas perturbações nas restrições de igualdade do problema. 
A abordagem é baseada nas informações de segunda ordem e nas condições de otimalidade.

A metodologia consiste, primeiramente, na obtenção de uma solução ótima inicial para o caso base via o FPO, e na utilização da sensibilidade para estimar novas soluções depois de ocorridas pequenas perturbações na demanda do sistema. Essas perturbações são um acréscimo ou um corte de carga em uma ou mais barras do sistema. A SFPO obteve novos pontos de operação para o sistema, sem a necessidade de se reprocessar o programa de FPO.

A matriz Lagrangiana e a matriz sensibilidade apresentam estruturas esparsas. Essa característica foi explorada na implementação computacional. Verificamos que, em sistemas elétricos reais, o "grau de esparsidade" dessas matrizes cresce conforme aumentam as dimensões das redes elétricas. Em virtude dessa esparsidade, o armazenamento das matrizes foi feito de forma compacta, isto é, somente os elementos diferentes de zero foram armazenados. Aplicamos as técnicas de esparsidade MA57 e MA28 aos problemas de FPO e de SFPO respectivamente. As sub-rotinas demonstraram um bom desempenho nos testes realizados, obtendo ganho de memória e robustez no processamento.

As principais contribuições do método em comparação com os programas de FPO são:

1) Método de resolução não iterativo; 
2) A não necessidade de se utilizarem parâmetros iniciais e de correção, como barreira e penalidade, utilizados nos programas de FPO convencionais;

3) Possibilidade de o método ser aplicado por usuários não especialistas em otimização.

Essas características fazem com que a aplicação do método possa ser viável para as empresas de energia elétrica.

Os resultados numéricos apresentados neste trabalho evidenciam o potencial da metodologia SFPO, para a resolução de problemas de FPO perturbado. Os testes demonstraram que a metodologia pode ser aplicada após pequenas perturbações nas restrições de igualdades.

A pesquisa desenvolvida leva-nos a uma sequiência de trabalhos futuros, enunciados a seguir:

1) Modelagem da metodologia SFPO com a introdução das restrições de fluxo nas linhas e tap's de transformadores;

2) Introdução de perturbações nas restrições de desigualdade e na função objetivo;

3) Realização de testes mais elaborados com sistemas elétricos maiores;

4) Modelagem do problema com a inclusão de outras funções objetivo. 


\section{Referências Bibliográficas}

ALTIMARI, M. M. R. S. (1999). Uma Estratégia Ótima do Despacho de Potência Ativa AC com Restrição na Transmissão, 157 p. Dissertação (Mestrado) EESC/SEL/LOSEP, USP.

AUMULlER, C.; SARA, T. K. (2002). Analysis and Assessment of Large Scale Power System Voltage Stability by a Novel Sensitivity Based Method. Power Engineering Society Summer Meeting, IEEE. v.3 p. 1621-1626.

BAPTISTA, E. C. (2001). Método da Função Lagrangiana Aumentada-Barreira Logarítmica para Solução do Problema de Fluxo de Potência Ótimo, 175 p. Tese (Doutorado) - EESC/SEL/LOSEP, USP.

BELL, K. R. W.; KIRSCHEN, D. S. (2000). Improved Sensitivities in MW Dispatch for Control of Voltage, IEEE Transactions on Power Apparatus and Systems, v.15, n. 3, p.1034-40, August.

CARPENTIER, J. L. (1962). Contribution a L'etude du Dispatching Economique. Bull-Soc. Fr. Elec. Ser. B3. p. 431-447. 
COSTA, G. R. M. (1990). O Método Dual-Newton Aplicado ao Fluxo de Carga Ótimo, Campinas. 235p. Tese (Doutorado) - FEE/DSCE, Unicamp.

COSTA, G. R. M. (1997). Optimal Reactive Dispatch Through Primal-Dual Method. IEEE Transactions on Power Systems, v. 12, n. 2, p.669 - 674, May.

COSTA, G. R. M.; COSTA, C. E. U. (2000). Improved Newton Method for Optimal Power Flow Problem. Electrical power and Energy Systems, v. 22, p. 459-462.

DOMMEL, H. W; TINNEY, W. F. (1968). Optimal Power Flow Solutions. IEEE Transactions on Power Apparatus and Systems, v.87, n.10, p.1866-76, Oct.

DUFF, I. S.; REID, J. K. (1979). Some Design Features of a Sparse Matrix Code. ACM Transactions on Mathematical Software, v. 5, n.1, March, p. 18-35.

EISENSTAT, C. S.; SCHULTZ, M. H.; SHERMAN, A. H. (1981). Algorithms and Data Structures for Sparse Symmetric Gaussian Elimination. Siam J. Sci. Stat. Compute. - v. 2, n.2, June, Society for Industrial and Applied Mathematics.

FANG, W.; NGAN, H. W. (2000). A Robust Load Flow Technique for Use in Power Systems with Unified Power Flow Controllers, Electric Power Systems Research, v.53, p. 181-186, March. 
FIACCO, A. V. (1976). Sensitivity Analysis for Nonlinear Programming Using Penalty Methods. Mathematical Programming 10(3), p. 278-311.

FIACCO, A. V. (1983). Introduction to Sensitivity and Stability Analysis in Nonlinear Programming. Vol. 165 in Mathematics in Science and Engineering Series, Academic Press, New York.

GRANVILLE, S. (1994). Optimal Reactive Dispatch Through Interior Point Methods. IEEE Transactions on Power Systems, v. 9, p. 136-146. February.

GRIBIK, P. R.; SHIRMOHAMMADI, D.; HAO, S.; THOMAS, C. L. (1990). Optimal Power Flow Sensitivity Analysis. IEEE Transactions on Power Systems, v.5, n.3, p 969-976, August.

GURRAN, R.; SUBRAMANYAM, B. (1999). Sensitivity Analysis of Radial Distribution Network - Adjoint Network Method. Electrical Power and Energy Systems, 21 (1999), p. 323-326.

HESTENES, M. R. (1969). SURVEY PAPER - Multiplier and Gradient Methods. Journal of Optimization Theory and Applications, v. 4, n.5, p.303-320.

JAN, R. M; CHEN, N. M. (1995). Application of Fast Newton-Raphson EconomicDispatch and Reactive Power Voltage Dispatch by Sensitivity Factors to 
Optimal Power-Flow. IEEE Transactions on Energy Conversion, v.10, n.2, p.293-301, June.

KISHORE, A.; HILL, E. F. (1971). Static Optimization of Reactive Power Sources by Use of Sensitivity Parameters. IEEE Transactions on Power Apparatus and Systems, v.90, n.3, p.1166-73, May.

MARKOWITZ, H. M. (1957). The elimination form of the inverse and its application to linear programming. Management Science, v.3, p.255-256.

MOMOH J, A.; ZHU J. Z. (1999). Improved Interior Point Method for OPF Problems. IEEE Transactions on Power Systems, v.14, p. 1114-1120, August.

MONTICELLI, A. (1983). Fluxo de Carga em Redes de Energia Elétrica. São Paulo, Edgard Blucher Ltda. Cap. 5, p. 75-100.

MONTICELLI, A.; LIU, W. H. E. (1992). Adaptative Movement Penalty Method for Newton Optimal Power Flow. IEEE Transactions on Power Systems, v.7, n.1, p.334-342. February.

NOBUO, S.; TINNEY, W. F. (1963). Techniques for Exploiting the Sparsity of the Network Admittance Matrix. AIEE Power System Engineering, v.1, p.944-50, December. 
PESCHON, J; PIERCY, D. S.; TINNEY, W. F.; TVEIT, O. J. (1968). Sensitivity in Power Systems. IEEE Transactions on Power Apparatus and Systems, v.87, n.8, p.1687-95, February.

QUINTANA, V. H.; GOMEZ, A.; MARTINEZ, J. L. (1995). Nonlinear Optimal Power Flows by a Logarithmic-Barrier Primal-Dual algorithm . In Proceedings IEEE North American Power Symposium, Bozeman, MO. P 178-185 October.

RASHED, A. M. H.; KELLY, D. H. (1974). Optimal Load Flow Solution Using Lagrangian Multipliers and the Hessian Matrix. IEEE Transactions on Power Apparatus and Systems, v. 93, n. 5, p. 1292-1297.

SANTOS, A.; DECKMAN, S.; SOARES, S. (1988). A Dual Lagrangian Approach for Optimal Power Flow. IEEE Transactions on Power Systems, v. 3, p. 10201025.

SASSON, A. M. (1969). Combined Use of the Powell and Fletcher-Powell Nonlinear Programming Load Flow Method. IEEE Transactions on Power Apparatus and Systems, v.88, p.1530-37.

SASSON, A.M.; VILORIA, F.; ABOYTES, F. (1973). Optimal Load Flow Solutions Using the Hessian Matrix. IEEE Transactions on Power Apparatus and Systems, v. 92, p. 31-41. 
SMED, T. (1993). Feasible Eigenvalue Sensitivity for Large Power-Systems. IEEE Transactions on Power Systems, v.8, n.2, p.555-63, May.

SUN, D. I.; ASHLEY, B.; BREWER, B.; HUGUES, A.; TINNEY, W. F. (1984). Optimal Power Flow by Newton Approach. IEEE Transactions on Power Apparatus and Systems, v. pas 103, n. 10, p. 2864-2875.

TINNEY, W. F.; WALKER, J. W. (1967). Direct Solutions of Sparse Network Equations by Optimally Ordered Triangular Factorization. Power Industry Computer Applications Conf., p. 367-76, February.

TINNEY, W. F; BRANDWAJN, V.; CHAN, S. M. (1985). Sparse Vector Methods. IEEE Transactions on Power Apparatus and Systems, v. pass-104, n.2, p.295301, February.

TORRES, G. L.; QUINTANA, V. H. (1998). Optimal Power Flow in Rectangular Form via an Interior Point Method. IEEE Transactions on Power Systems, v. 13, n. 4, p. 1211-1218.

WRIGHT, M. H. (1995). Why A Pure Primal Newton Barrier Step May Be Infeasible. SIAM Journal on Optimization, v. 5, n. 1, p. 1-12.

WU, YU-CHI Y.; DEBS, A. S.; MARSTEN, R. E. (1994). A Direct Nonlinear Predictor-Corrector Primal-Dual Interior Point Algorithm for Optimal Power 
Flows. IEEE Transactions on Power Systems, v. 9, p. 876-883 May.

ZOLLENKOPF, K. (1971). Basic Computational Algorithm and Programming Techniques. J. K. Reid ed. - Large Sparse sets Linear Equations, p. 75-97, New York Academic Press. 
Apêndice A

\section{BANCO DE DADOS E TABELAS DE CONVERGÊNCIA DOS SISTEMAS ELÉTRICOS UTILIZADOS}

Neste apêndice estão apresentados os bancos de dados e as Tabelas com informações da convergência dos sistemas elétricos. As Tabelas contêm os parâmetros iniciais, o número de iterações internas e externas e as perdas de potência ativa na transmissão. Os bancos de dados apresentados estão no formato PECO (Philadelphia Electric Company).

\section{A.1 TABELAS DE CONVERGÊNCIA DOS SISTEMAS ELÉTRICOS UTILIZADOS}

TABELA A.1 - Convergência do sistema 3 Barras

\begin{tabular}{|c|c|c|c|c||c||c|}
\hline $\mathbf{c}$ & $\boldsymbol{\beta}$ & $\boldsymbol{\delta}$ & $\boldsymbol{\rho}$ & It. Int. & It. Ext. & Perdas(MW) \\
\hline \hline 1,00 & 1,10 & 0,001 & 10 & 12 & 9 & 12,916 \\
\hline
\end{tabular}


TABELA A.2 - Convergência do sistema IEEE14 Barras

\begin{tabular}{|c||c||c||c||c||c||c|}
\hline $\mathbf{c}$ & $\boldsymbol{\beta}$ & $\boldsymbol{\delta}$ & $\boldsymbol{\rho}$ & It. Int. & It. Ext. & Perdas(MW) \\
\hline \hline 1,00 & 1,20 & 0,001 & 10 & 15 & 12 & 12,256 \\
\hline
\end{tabular}

TABELA A.3 - Convergência do sistema IEEE 30 Barras

\begin{tabular}{|c||c||c|c||c||c||c|}
\hline $\mathbf{c}$ & $\boldsymbol{\beta}$ & $\boldsymbol{\delta}$ & $\boldsymbol{\rho}$ & It. Int. & It. Ext. & Perdas(MW) \\
\hline \hline 1,00 & 1,15 & 0,0001 & 10 & 7 & 5 & 16,279 \\
\hline
\end{tabular}

TABELA A.4 - Convergência do sistema CESP

\begin{tabular}{|c|c|c||c||c||c||c|}
\hline $\mathbf{c}$ & $\boldsymbol{\beta}$ & $\boldsymbol{\delta}$ & $\boldsymbol{\rho}$ & It. Int. & It. Ext. & Perdas(MW) \\
\hline \hline 1,00 & 1,01 & 0,01 & 10 & 16 & 10 & 213,45 \\
\hline
\end{tabular}

TABELA A.5 - Convergência do sistema IEEE 118 Barras

\begin{tabular}{|c|c|c|c|c|c|c|}
\hline $\mathbf{c}$ & $\boldsymbol{\beta}$ & $\boldsymbol{\delta}$ & $\boldsymbol{\rho}$ & It. Int. & It. Ext. & Perdas(MW) \\
\hline \hline 1,00 & 1,2 & 0,0001 & 10 & 14 & 10 & 131,56 \\
\hline
\end{tabular}

TABELA A.6 - Convergência do sistema IEEE 162 Barras

\begin{tabular}{|c|c|c|c|c||c||c|}
\hline $\mathbf{c}$ & $\boldsymbol{\beta}$ & $\boldsymbol{\delta}$ & $\boldsymbol{\rho}$ & It. Int. & It. Ext. & Perdas(MW) \\
\hline \hline 1,00 & 1,12 & 0,001 & 10 & 15 & 14 & 158,30 \\
\hline
\end{tabular}

TABELA A.7- Convergência do sistema IEEE 300 Barras

\begin{tabular}{|c||c|c||c|c||c||c|}
\hline \hline $\mathbf{c}$ & $\boldsymbol{\beta}$ & $\boldsymbol{\delta}$ & $\boldsymbol{\rho}$ & It. Int. & It. Ext. & Perdas(MW) \\
\hline \hline 1,0 & 1,001 & 0,00001 & 10 & 18 & 16 & 408,31 \\
\hline
\end{tabular}

\section{A.2 BANCO DE DADOS DOS SISTEMAS ELÉTRICOS}

\section{UTILIZADOS}

\section{A.2.1 BANCO DE DADOS DO SISTEMA 9 BARRAS}

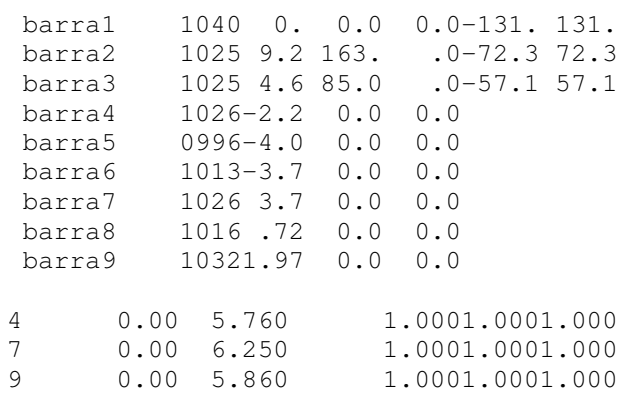




$\begin{array}{rrrrrr}4 & 5 & 1.00 & 8.500 & 17.6 & 900 \\ 4 & 6 & 1.70 & 9.200 & 15.8 & 900 \\ 5 & 7 & 3.20 & 16.100 & 30.6 & 900 \\ 6 & 9 & 3.90 & 17.000 & 35.8 & 900 \\ 7 & 8 & 0.85 & 7.200 & 14.9 & 900 \\ 8 & 9 & 1.19 & 10.080 & 20.9 & 900 \\ 9999 & & & & & \\ 9999 & 0.95 & 1.1 & & & \end{array}$

\section{A.2.2 BANCO DE DADOS DO SISTEMA IEEE 14 BARRAS}

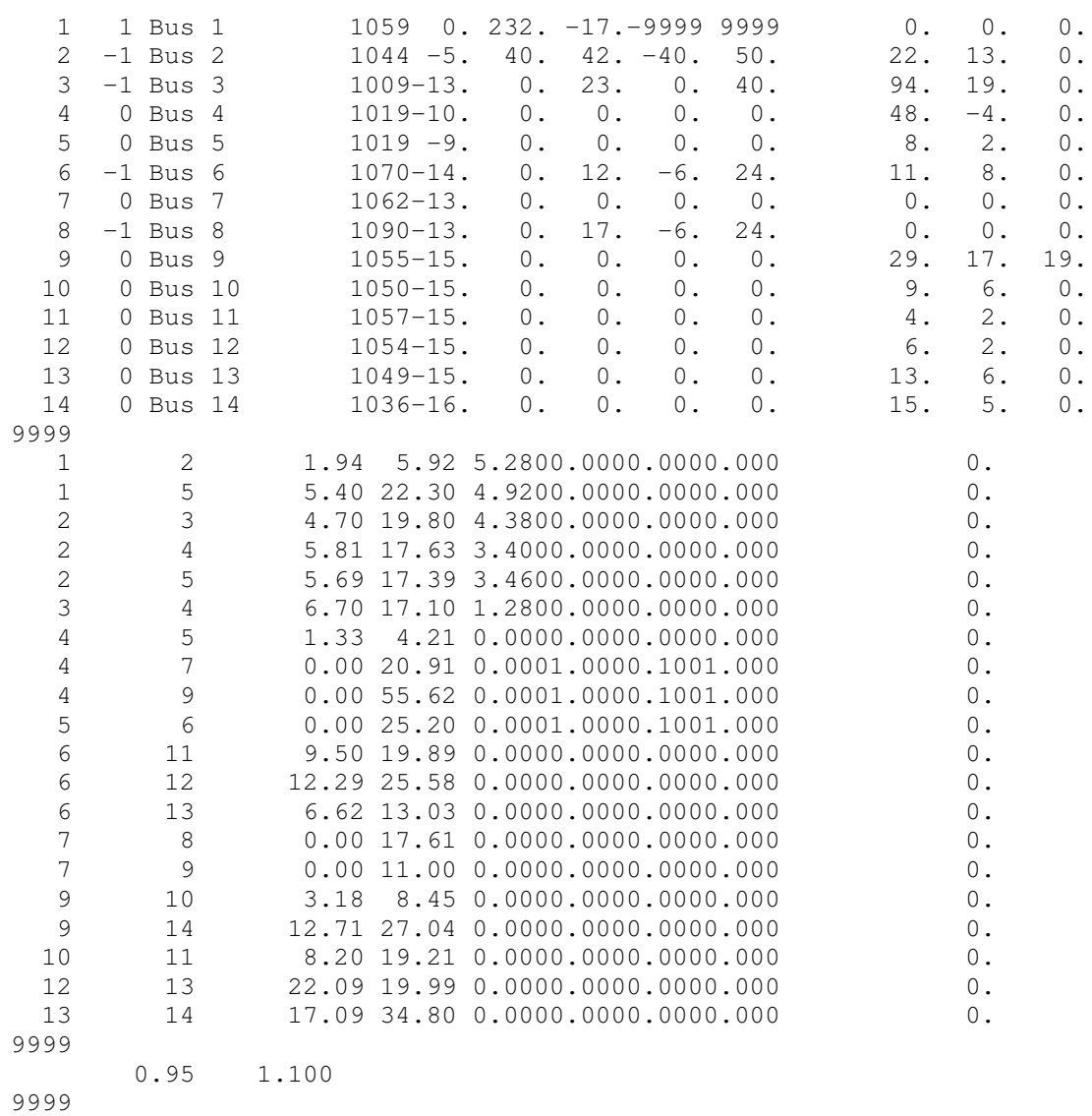

\section{A.2.3 BANCO DE DADOS DO SISTEMA IEEE 30 BARRAS}

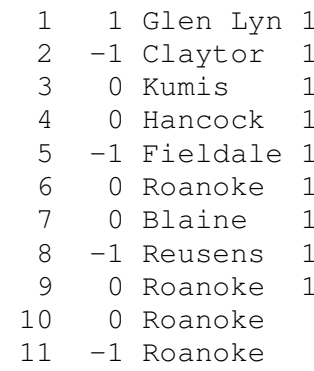

\begin{tabular}{|c|c|c|c|c|}
\hline 10590. & 260. & -16. & -9999 & 99 \\
\hline $1042-5$. & 40. & 50. & -40 . & \\
\hline $1021-8$. & 0. & 0 . & 0 . & \\
\hline 1011-10. & 0 . & 0 . & 0 . & \\
\hline $009-14$. & 0. & 37. & -40 . & \\
\hline $009-11$. & 0 . & 0 & 0 & \\
\hline $001-13$. & 0 . & 0 . & 0 . & \\
\hline $009-12$. & 0 . & 37. & -10 & \\
\hline 50-14. & 0 . & 0 & 0 & \\
\hline $44-16$. & 0 . & 0 . & 0 & \\
\hline $32-14$ & 0 . & 16. & -6 & \\
\hline
\end{tabular}

$\begin{array}{rrr}0 . & 0 . & 0 . \\ 22 . & 13 . & 0 . \\ 2 . & 1 . & 0 . \\ 8 . & 2 . & 0 . \\ 94 . & 19 . & 0 . \\ 0 . & 0 . & 0 . \\ 23 . & 11 . & 0 . \\ 30 . & 30 . & 0 . \\ 0 . & 0 . & 0 . \\ 6 . & 2 . & 19 . \\ 0 . & 0 . & 0 .\end{array}$




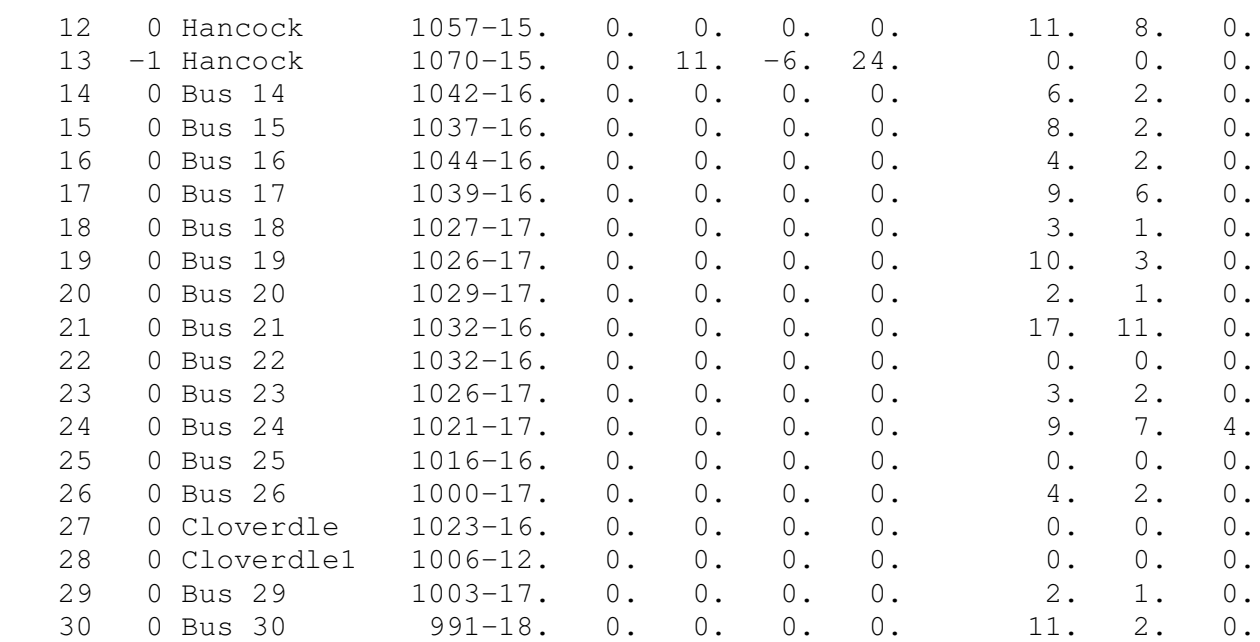

9999

$$
\begin{array}{r}
99 \\
1
\end{array}
$$

1
1

999

9999
$1.92 \quad 5.75 \quad 5.2800 .0000 .0000 .000$ 4.5216 .524 .0800 .0000 .0000 .000 $5.7017 .37 \quad 3.6800 .0000 .0000 .000$ $1.32 \quad 3.790 .8400 .0000 .0000 .000$ 4.7219 .834 .1800 .0000 .0000 .000 $5.8117 .63 \quad 3.7400 .0000 .0000 .000$ $\begin{array}{lll}1.19 & 4.14 & 0.9000 .0000 .0000 .000\end{array}$ 4.6011 .602 .0400 .0000 .0000 .000 2.678 .201 .7000 .0000 .0000 .000 $1.20 \quad 4.20 \quad 0.9000 .0000 .0000 .000$ $0.0020 .80 \quad 0.0000 .0000 .0000 .000$ $0.0055 .60 \quad 0.0000 .0000 .0000 .000$ $0.0020 .80 \quad 0.0000 .0000 .0000 .000$ $0.0011 .00 \quad 0.0000 .0000 .0000 .000$ $0.0025 .60 \quad 0.0000 .0000 .0000 .000$ $0.0014 .00 \quad 0.0000 .0000 .0000 .000$ 12.3125 .590 .0000 .0000 .0000 .000 6.6213 .040 .0000 .0000 .0000 .000 9.4519 .870 .0000 .0000 .0000 .000 22.1019 .970 .0000 .0000 .0000 .000 $5.2419 .23 \quad 0.0000 .0000 .0000 .000$ 10.7321 .850 .0000 .0000 .0000 .000 6.3912 .920 .0000 .0000 .0000 .000 $\begin{array}{lll}3.40 & 6.80 & 0.0000 .0000 .0000 .000\end{array}$ 9.3620 .900 .0000 .0000 .0000 .000 3.248 .450 .0000 .0000 .0000 .000 $3.48 \quad 7.490 .0000 .0000 .0000 .000$ $\begin{array}{lll}7.27 & 14.99 & 0.0000 .0000 .0000 .000\end{array}$ 1.162 .360 .0000 .0000 .0000 .000 $10.0020 .20 \quad 0.0000 .0000 .0000 .000$ $11.5017 .90 \quad 0.0000 .0000 .0000 .000$ $13.2027 .00 \quad 0.0000 .0000 .0000 .000$ 18.8532 .920 .0000 .0000 .0000 .000 $25.4438 .00 \quad 0.0000 .0000 .0000 .000$ 10.9320 .870 .0000 .0000 .0000 .000 $0.00 \quad 39.60 \quad 0.0000 .0000 .0000 .000$ $21.9841 .53 \quad 0.0000 .0000 .0000 .000$ $32.02 \quad 60.27 \quad 0.0000 .0000 .0000 .000$ 23.9945 .330 .0000 .0000 .0000 .000 $6.3620 .00 \quad 4.2800 .0000 .0000 .000$ 1.695 .991 .3000 .0000 .0000 .000 0.95 1.100 


\section{A.2.4 BANCO DE DADOS DO SISTEMA CESP 53 BARRAS}

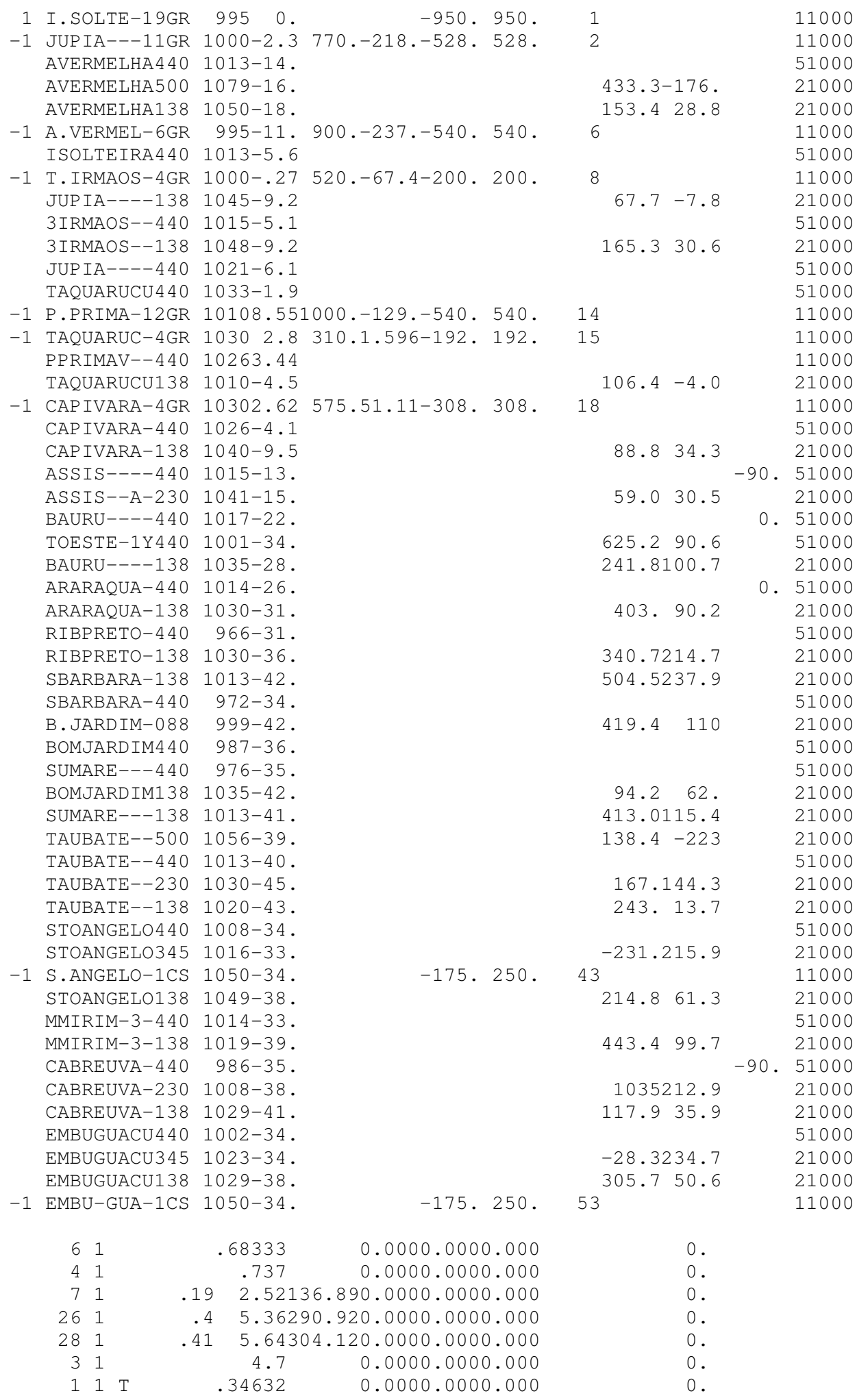




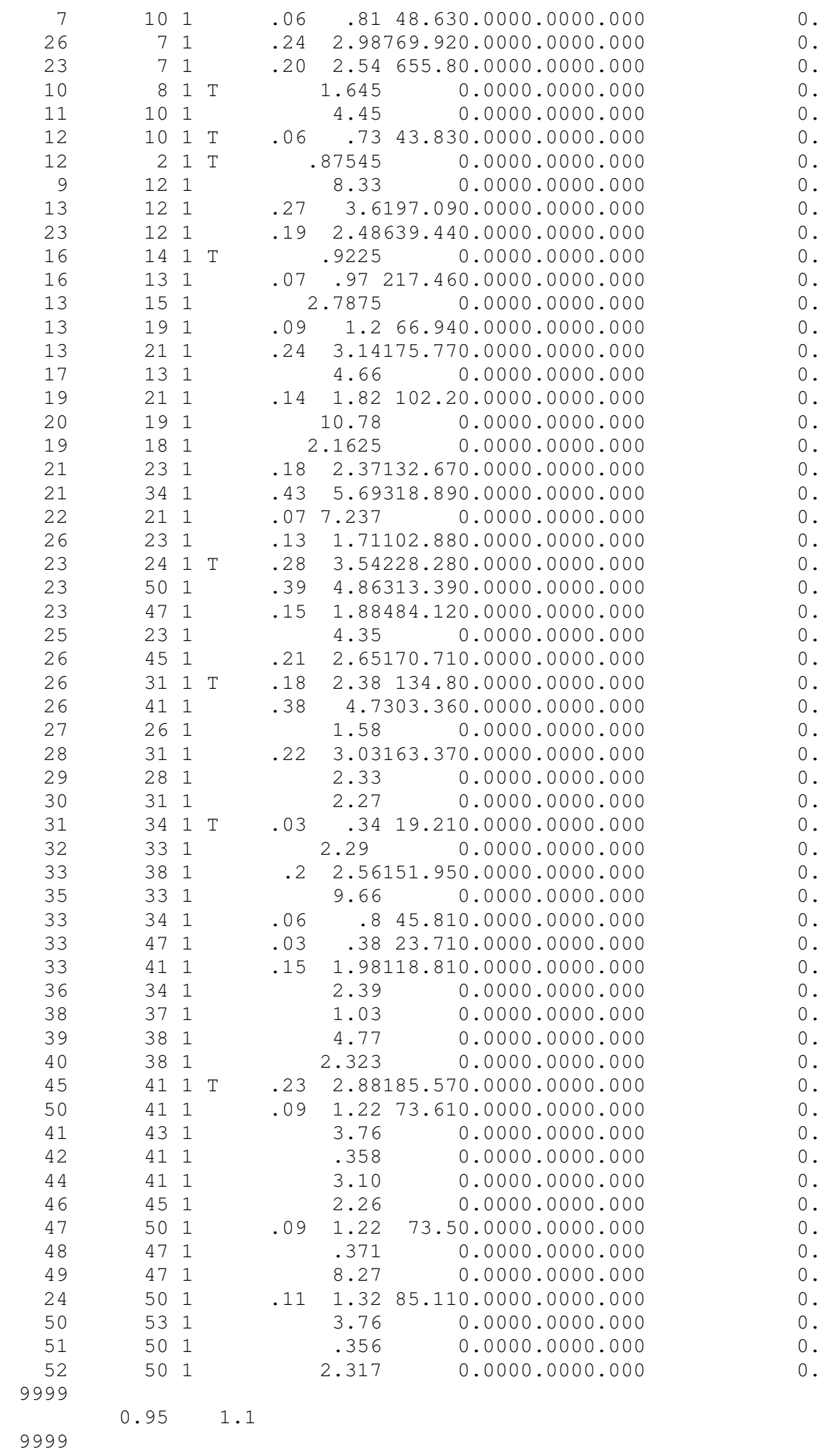




\section{A.2.5 BANCO DE DADOS DO SISTEMA IEEE 118 BARRAS}

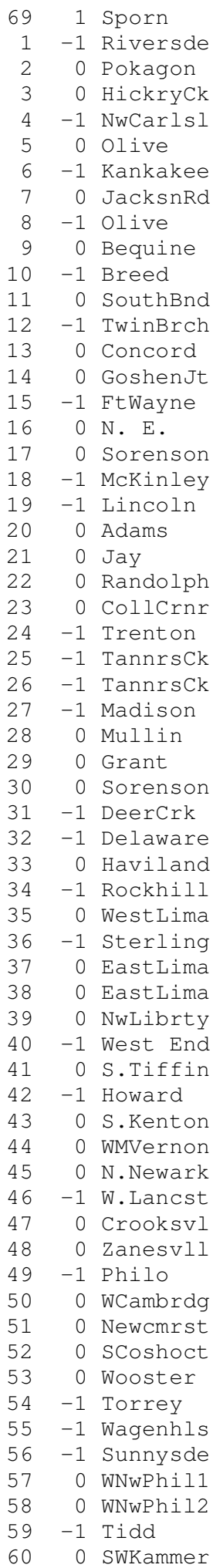

\begin{tabular}{|c|c|c|c|c|}
\hline .034 & 30. & 516. & $0 .-9999$ & 9999. \\
\hline 954 & 11. & 0 . & 0. $\quad-5$. & 15. \\
\hline 971 & 11. & 0 . & 0. & 0 \\
\hline 967 & 12 . & 0 . & 0 . & 0. \\
\hline 998 & 15. & -9 . & $0 .-300$. & 300 . \\
\hline 1001 & 16. & 0 & $0 . \quad 0$. & 0 \\
\hline 990 & 13. & 0 . & $0 .-13$. & 50. \\
\hline 989 & 13. & 0 . & $0 . \quad 0$. & 0 \\
\hline 1014 & 21 . & -28 & $0 .-300$. & 300 . \\
\hline 1042 & 28. & 0 & $0 . \quad 0$. & 0 \\
\hline 1049 & 36. & 450. & $0 .-147$. & 200 \\
\hline 985 & 13. & 0 & $0 . \quad 0$. & \\
\hline 990 & 12 . & 85 . & $0 .-35$. & 120. \\
\hline 967 & 11. & 0 & $0 . \quad 0$. & 0 \\
\hline 984 & 11. & 0 . & 0 . & 0 . \\
\hline 970 & 11. & 0 . & $0 .-10$. & 30. \\
\hline 984 & 12. & 0 . & $0 . \quad 0$. & 0 \\
\hline 995 & 14. & 0 . & 0. & \\
\hline 972 & 12. & 0 . & $0 .-16$. & \\
\hline 962 & 11. & 0 . & 0. $\quad-8$. & 24. \\
\hline 958 & 12. & 0 . & 0. & 0 \\
\hline 958 & 14. & 0 . & 0 . & 0 . \\
\hline 970 & 16. & 0 . & 0 . & 0 . \\
\hline 1000 & 21 . & 0 . & 0. & 0 . \\
\hline 991 & 21 . & -13. & $0 .-300$. & 300. \\
\hline 1049 & 28. & 220 . & 0. -47 . & 140. \\
\hline 1014 & 30 . & 314. & $0 .-1000$ & .1000 \\
\hline 967 & 15. & -9 & $0 .-300$. & 300 \\
\hline 962 & 14. & 0 & $0 . \quad 0$. & 0 \\
\hline 962 & 13. & 0 . & 0 . & 0 \\
\hline 967 & 19. & 0 . & 0 . & 0 \\
\hline 967 & 13. & 7 . & $0 .-300$. & 300. \\
\hline 963 & 15 . & 0 . & $0 .-14$. & 42. \\
\hline 972 & 11. & 0 . & 0 . & 0 \\
\hline 986 & 11. & 0 . & -8 & 24. \\
\hline 981 & 11. & 0 . & 0 . & 0 \\
\hline 980 & 11. & 0 . & -8 & 24. \\
\hline 991 & 12. & 0 . & 0 . & 0 \\
\hline 962 & 17. & 0 . & 0 . & 0. \\
\hline 970 & 8. & 0 . & 0 & 0 . \\
\hline 970 & 7 . & -46 & $0 .-300$. & 300. \\
\hline 967 & 7 . & 0 . & $0 . \quad 0$. & 0 \\
\hline 985 & 9. & -59. & $0 .-300$. & 300 . \\
\hline 977 & 11. & 0 . & $0 . \quad 0$. & 0. \\
\hline 985 & 14. & 0 . & 0 . & 0 . \\
\hline 986 & 16. & 0 . & 0 . & 0. \\
\hline 1004 & 18. & 19. & $0 .-100$. & 100. \\
\hline 1016 & 21 . & 0 & $0 . \quad 0$. & 0 \\
\hline 1021 & 20 . & 0 & 0. & 0 \\
\hline 1024 & 21 . & 204 . & $0 .-85$. & 210 \\
\hline 1001 & 19. & 0 & $0 . \quad 0$. & 0 \\
\hline 967 & 16. & 0 . & 0 . & 0 \\
\hline 957 & 15. & 0 . & 0 . & 0 \\
\hline 945 & 14. & 0 . & 0 . & 0 \\
\hline 954 & 15. & 48. & $0 .-300$. & 300 \\
\hline 952 & 15. & 0 & $0 . \quad-8$. & 23 \\
\hline 953 & 15. & 0 . & -8 & 15 \\
\hline 971 & 16. & 0 . & 0 . & 0 \\
\hline 958 & 16. & 0 . & 0 & 0 \\
\hline 985 & 19. & 155. & 0. -60 . & 180 \\
\hline 992 & & 0 & $0 . \quad 0$. & \\
\hline
\end{tabular}

\begin{tabular}{|c|c|c|}
\hline 0. & 0 . & 0 \\
\hline 51. & 27. & 0 \\
\hline 20. & 9. & \\
\hline 39. & 10. & \\
\hline 30. & 12. & \\
\hline 0. & 0 & -40 \\
\hline 52. & 22. & \\
\hline 19. & 2 & \\
\hline 0. & 0. & \\
\hline 0 . & 0. & \\
\hline 0. & 0 & \\
\hline 70. & 23. & \\
\hline 47. & 10. & \\
\hline 34. & 16. & \\
\hline 14. & 1 & \\
\hline 90. & 30. & \\
\hline 25. & 10. & \\
\hline 11. & 3 & \\
\hline 60. & 34. & \\
\hline 45. & 25. & \\
\hline 18. & 3. & \\
\hline 14. & 8. & \\
\hline 10. & 5. & \\
\hline 7. & 3. & \\
\hline 0 . & 0 . & \\
\hline 0. & 0 . & \\
\hline 0. & 0 . & \\
\hline 62. & 13. & \\
\hline 17. & 7. & \\
\hline 24. & 4. & \\
\hline 0. & 0 & \\
\hline 43. & 27. & \\
\hline 59. & 23. & \\
\hline 23. & 9. & \\
\hline 59. & 26. & 14 \\
\hline 33. & 9. & \\
\hline 31. & 17. & \\
\hline 0. & 0 & -25 \\
\hline 0. & 0 . & \\
\hline 27. & 11. & \\
\hline 20. & 23. & \\
\hline 37. & 10. & \\
\hline 37. & 23. & \\
\hline 18. & 7. & \\
\hline 16. & 8. & \\
\hline 53. & 22. & 10 \\
\hline 28. & 10. & 10 \\
\hline 34. & 0 . & \\
\hline 20. & 11. & 15 \\
\hline 87. & 30. & \\
\hline 17. & 4. & \\
\hline 17. & 8. & \\
\hline 18. & 5. & \\
\hline 23. & 11. & \\
\hline 113. & 32. & \\
\hline 63. & 22 . & \\
\hline 84. & 18. & \\
\hline 12. & 3. & \\
\hline 12. & 3. & \\
\hline & & \\
\hline & & \\
\hline
\end{tabular}




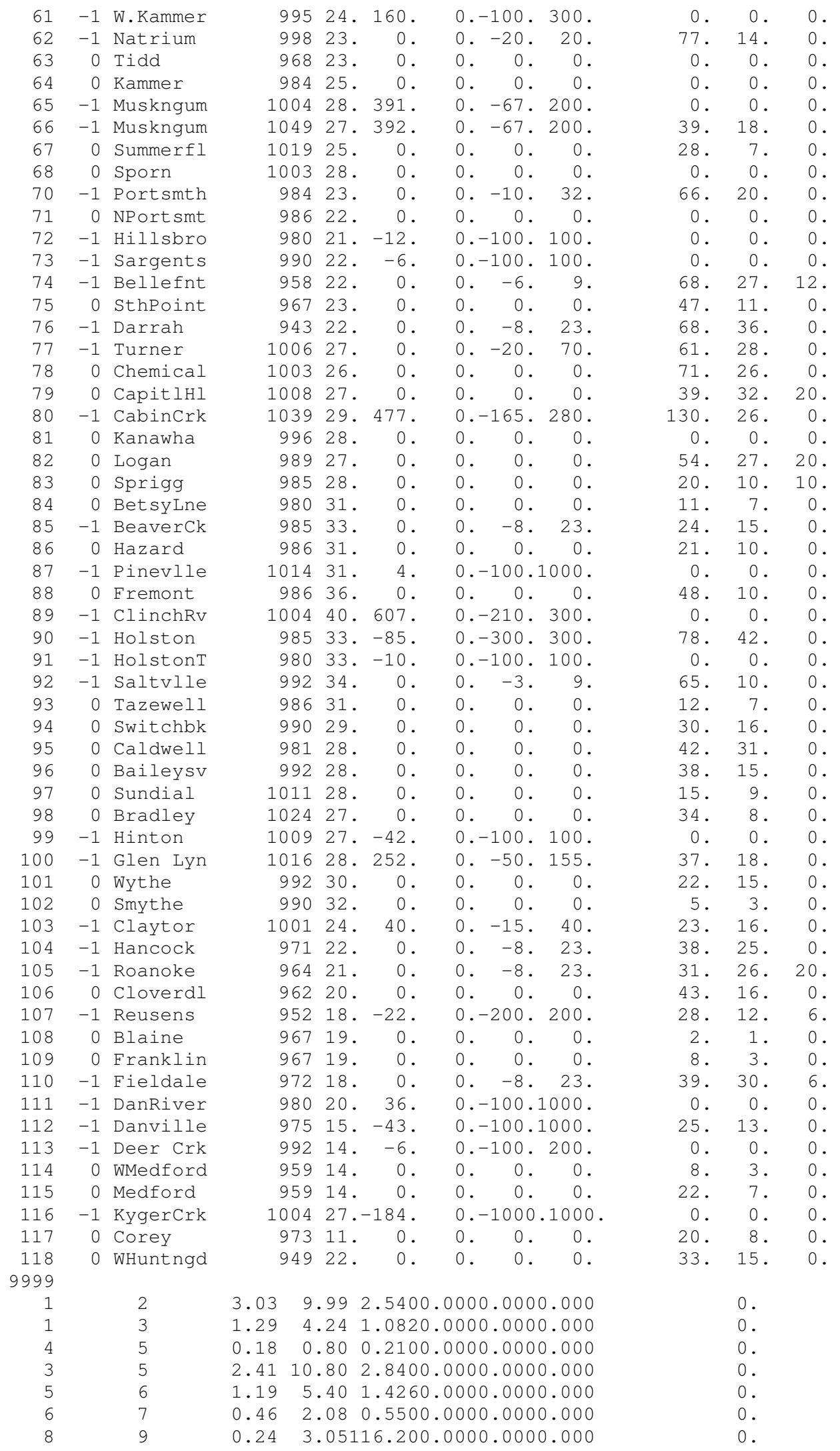




\begin{tabular}{|c|c|c|c|c|}
\hline 8 & 5 & 0.00 & 2.670 .0000 .0000 .0000 .000 & \\
\hline 9 & 10 & 0.26 & 3.22123 .000 .0000 .0000 .000 & \\
\hline 4 & 11 & 2.09 & 6.881 .7480 .0000 .0000 .000 & \\
\hline 5 & 11 & 2.03 & 6.821 .7380 .0000 .0000 .000 & \\
\hline 11 & 12 & 0.60 & 1.960 .5020 .0000 .0000 .000 & \\
\hline 2 & 12 & 1.87 & 6.161 .5720 .0000 .0000 .000 & \\
\hline 3 & 12 & 4.84 & 16.004 .0600 .0000 .0000 .000 & \\
\hline 7 & 12 & 0.86 & 3.400 .8740 .0000 .0000 .000 & \\
\hline 11 & 13 & 2.23 & 7.311 .8760 .0000 .0000 .000 & \\
\hline 12 & 14 & 2.15 & 7.071 .8160 .0000 .0000 .000 & \\
\hline 13 & 15 & 7.44 & $24.44 \quad 6.2680 .0000 .0000 .000$ & \\
\hline 14 & 15 & 5.95 & 19.505 .0200 .0000 .0000 .000 & \\
\hline 12 & 16 & 2.12 & 8.342 .1400 .0000 .0000 .000 & \\
\hline 15 & 17 & 1.32 & 4.374 .4400 .0000 .0000 .000 & \\
\hline 16 & 17 & 4.54 & 18.014 .6600 .0000 .0000 .000 & \\
\hline 17 & 18 & 1.23 & 5.051 .2980 .0000 .0000 .000 & \\
\hline 18 & 19 & 1.12 & 4.931 .1420 .0000 .0000 .000 & \\
\hline 19 & 20 & 2.52 & 11.702 .9800 .0000 .0000 .000 & \\
\hline 15 & 19 & 1.20 & 3.941 .0100 .0000 .0000 .000 & \\
\hline 20 & 21 & 1.83 & 8.492 .1600 .0000 .0000 .000 & \\
\hline 21 & 22 & 2.09 & 9.702 .4600 .0000 .0000 .000 & \\
\hline 22 & 23 & 3.42 & 15.904 .0400 .0000 .0000 .000 & \\
\hline 23 & 24 & 1.35 & 4.924 .9800 .0000 .0000 .000 & \\
\hline 23 & 25 & 1.56 & 8.008 .6400 .0000 .0000 .000 & \\
\hline 26 & 25 & 0.00 & 3.820 .0000 .0000 .0000 .000 & \\
\hline 25 & 27 & 3.18 & 16.3017 .6400 .0000 .0000 .000 & \\
\hline 27 & 28 & 1.91 & 8.552 .1600 .0000 .0000 .000 & \\
\hline 28 & 29 & 2.37 & 9.432 .3800 .0000 .0000 .000 & \\
\hline 30 & 17 & 0.00 & 3.880 .0000 .0000 .0000 .000 & \\
\hline 8 & 30 & 0.43 & 5.0451 .4000 .0000 .0000 .000 & \\
\hline 26 & 30 & 0.80 & 8.6090 .8000 .0000 .0000 .000 & \\
\hline 17 & 31 & 4.74 & 15.633 .9900 .0000 .0000 .000 & \\
\hline 29 & 31 & 1.08 & 3.310 .8300 .0000 .0000 .000 & \\
\hline 23 & 32 & 3.17 & 11.5311 .7300 .0000 .0000 .000 & \\
\hline 31 & 32 & 2.98 & 9.852 .5100 .0000 .0000 .000 & \\
\hline 27 & 32 & 2.29 & 7.551 .9260 .0000 .0000 .000 & \\
\hline 15 & 33 & 3.80 & 12.443 .1940 .0000 .0000 .000 & \\
\hline 19 & 34 & 7.52 & 24.706 .3200 .0000 .0000 .000 & \\
\hline 35 & 36 & 0.22 & 1.020 .2680 .0000 .0000 .000 & \\
\hline 35 & 37 & 1.10 & 4.971 .3180 .0000 .0000 .000 & \\
\hline 33 & 37 & 4.15 & 14.203 .6600 .0000 .0000 .000 & \\
\hline 34 & 36 & 0.87 & 2.680 .5680 .0000 .0000 .000 & \\
\hline 34 & 37 & 0.26 & 0.940 .9840 .0000 .0000 .000 & \\
\hline 38 & 37 & 0.00 & 3.750 .0000 .0000 .0000 .000 & \\
\hline 37 & 39 & 3.21 & 10.602 .7000 .0000 .0000 .000 & \\
\hline 37 & 40 & 5.93 & 16.804 .2000 .0000 .0000 .000 & \\
\hline 30 & 38 & 0.46 & 5.4042 .2000 .0000 .0000 .000 & \\
\hline 39 & 40 & 1.84 & 6.051 .5520 .0000 .0000 .000 & \\
\hline 40 & 41 & 1.45 & 4.871 .2220 .0000 .0000 .000 & \\
\hline 40 & 42 & 5.55 & 18.304 .6600 .0000 .0000 .000 & \\
\hline 41 & 42 & 4.10 & 13.503 .4400 .0000 .0000 .000 & \\
\hline 43 & 44 & 6.08 & 24.546 .0680 .0000 .0000 .000 & \\
\hline 34 & 43 & 4.13 & 16.814 .2260 .0000 .0000 .000 & \\
\hline 44 & 45 & 2.24 & 9.012 .2400 .0000 .0000 .000 & \\
\hline 45 & 46 & 4.00 & 13.563 .3200 .0000 .0000 .000 & \\
\hline 46 & 47 & 3.80 & 12.703 .1600 .0000 .0000 .000 & \\
\hline 46 & 48 & 6.01 & 18.904 .7200 .0000 .0000 .000 & \\
\hline 47 & 49 & 1.91 & 6.251 .6040 .0000 .0000 .000 & \\
\hline 42 & 49 & 3.57 & 16.1517 .2000 .0000 .0000 .000 & \\
\hline 45 & 49 & 6.84 & 18.604 .4400 .0000 .0000 .000 & \\
\hline 48 & 49 & 1.79 & 5.051 .2580 .0000 .0000 .000 & \\
\hline 49 & 50 & 2.67 & 7.521 .8740 .0000 .0000 .000 & \\
\hline 49 & 51 & 4.86 & 13.703 .4200 .0000 .0000 .000 & \\
\hline 51 & 52 & 2.03 & 5.881 .3960 .0000 .0000 .000 & \\
\hline 52 & 53 & 4.05 & 16.354 .0580 .0000 .0000 .000 & \\
\hline
\end{tabular}




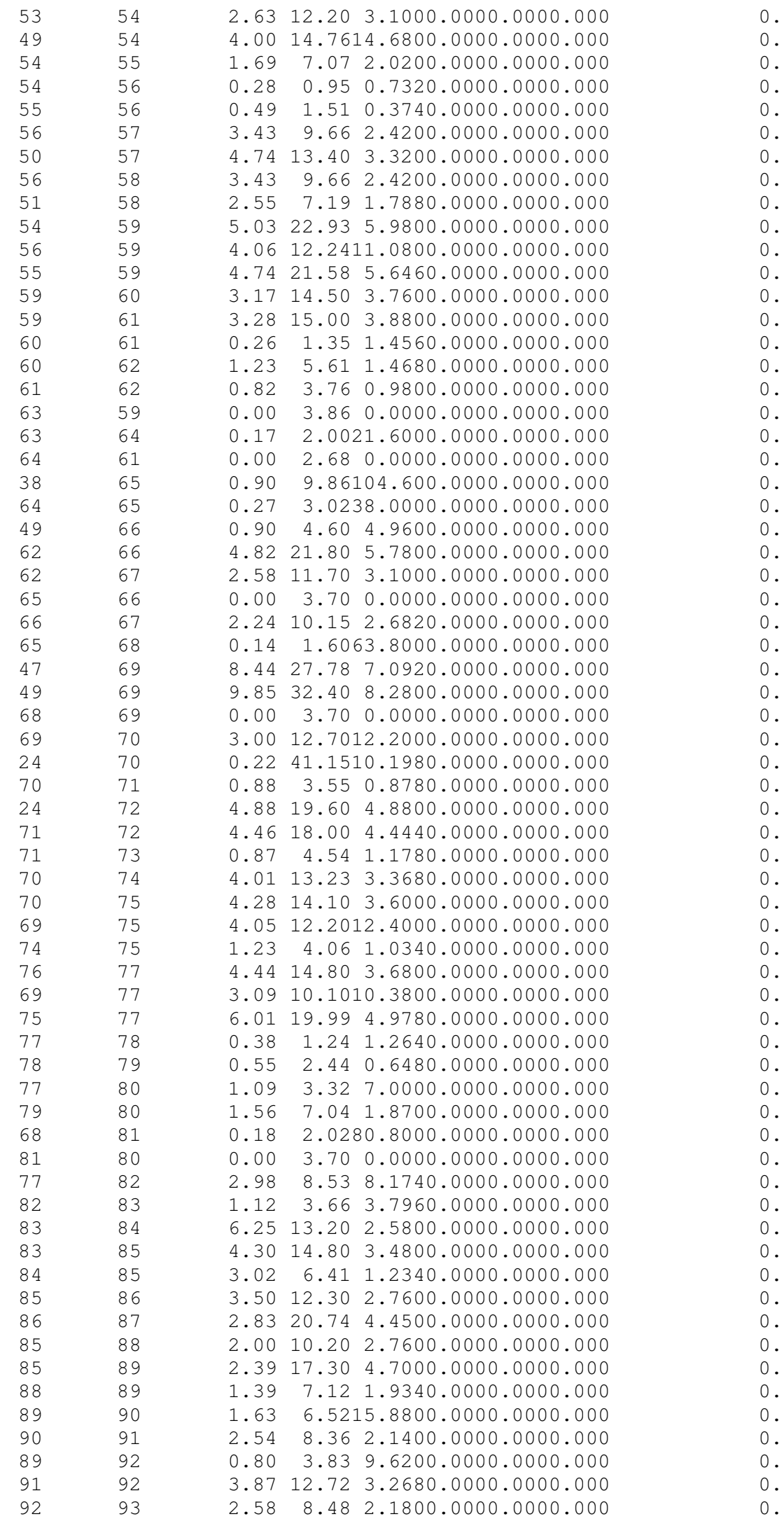




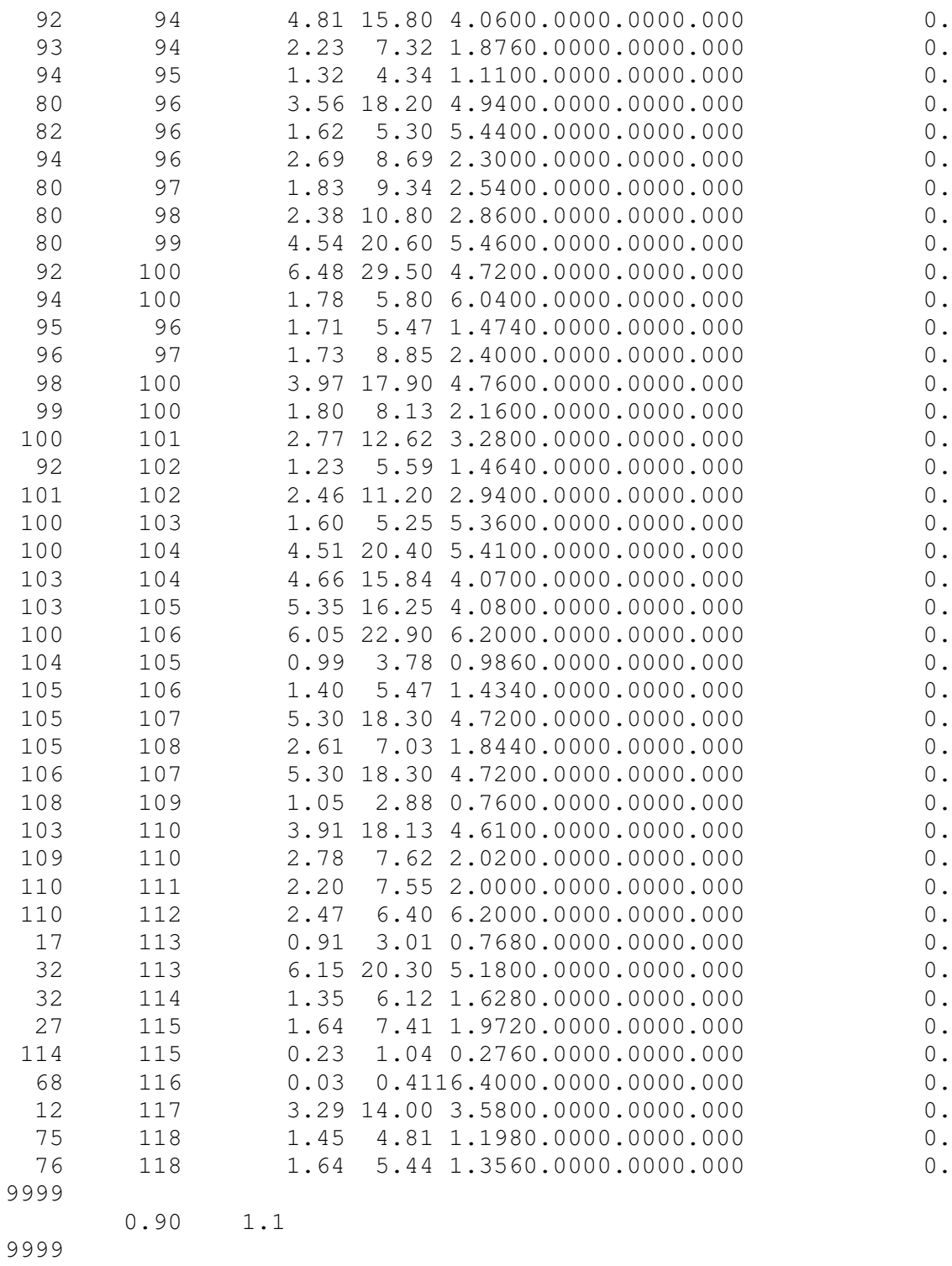

\section{A.2.6 BANCO DE DADOS DO SISTEMA IEEE 162 BARRAS}

\begin{tabular}{|c|c|c|c|c|c|c|}
\hline 108 & 1 & G & & $.000-28.551$. & $156 .-9999$ & 99999. \\
\hline 1 & 0 & 3 & 3 & 1032-25. 0 . & $0 . \quad 0$ & . 0 \\
\hline 2 & 0 & 3 & 3 & $1022-30$ & 0 . & . \\
\hline 3 & 0 & 2 & 1 & $999-32.2000$ & 0 . & . \\
\hline 4 & 0 & 3 & 3 & 1019-34. 0 . & 0 . & . \\
\hline 5 & 0 & 3 & 3 & $1033-25$. & $0 . \quad 0$ & . \\
\hline 6 & -1 & G & & $1000-19.794$. & $181 .-200$ & 40 \\
\hline 7 & 0 & 3 & 3 & 1018-30. 0 . & $0 . \quad 0$ & 0 . \\
\hline 8 & 0 & 7 & 1 & $1034-34 .-398$. & -19 & 0 . \\
\hline 9 & 0 & 3 & 3 & 1026-28. 0 . & 0 & b. \\
\hline 10 & 0 & 4 & 2 & $992-36 .-226$ & 11. & 0 . \\
\hline 11 & 0 & 4 & 2 & 999-32. 0 & 0 & 0 . \\
\hline 12 & 0 & 7 & 1 & $1037-34 .-193$. & 6. & 0 . \\
\hline 13 & 0 & 3 & 3 & $1014-31 .-204$. & -37 & 0 . \\
\hline 14 & 0 & 5 & 1 & $1027-31 .-381$. & -56 & 0. \\
\hline 15 & 0 & 4 & 2 & $1018-25.1500$. & 6 & 0. \\
\hline
\end{tabular}

$\begin{array}{rrr}0 . & 0 . & 0 . \\ 0 . & 0 . & -100 . \\ 0 . & 0 . & 0 . \\ 2370 . & 97 . & 0 . \\ 0 . & 0 . & 0 . \\ 0 . & 0 . & -50 . \\ 0 . & 0 . & 0 . \\ 0 . & 0 . & 0 . \\ 0 . & 0 . & 0 . \\ 0 . & 0 . & 0 . \\ 0 . & 0 . & 0 . \\ 0 . & 0 . & 0 . \\ 0 . & 0 . & 0 . \\ 0 . & 0 . & 0 . \\ 0 . & 0 . & 0 . \\ 1420 . & 0 . & 0 .\end{array}$




\begin{tabular}{|c|c|c|c|c|c|c|c|c|c|}
\hline 5 & 1 & $1014-30$ & 54. & -27 & 0 . & 0. & 0 . & 0 & 0 . \\
\hline 5 & 1 & $1005-29$ & 116. & -45 & 0 . & 0 . & 0 & 0 & 0 . \\
\hline 5 & 1 & $1035-34$ & 0 . & 0 & 0 . & 0 . & 34. & 12 & 0 . \\
\hline 5 & 1 & 999-38. & -64 . & -4 & 0 . & 0 . & 0 & 0 & 0 . \\
\hline 8 & & 979-33 & 0 . & 0 & 0 . & 0 . & 38. & 12 & 0 . \\
\hline 5 & 1 & $1008-30$ & 70 . & -23 & 0 . & 0 . & 0 . & 0 & 0 . \\
\hline 5 & 1 & $1033-38$ & 0 . & 0 & 0 . & 0 . & 17. & 5 & 0 . \\
\hline 5 & 1 & $988-35$ & -63. & -21 & 0 . & 0 . & 0 & 0 & 0 . \\
\hline 5 & 1 & $1009-33$ & 0 . & 0 & 0 . & 0 . & 0 . & 0 & 0 . \\
\hline 3 & 3 & $1001-29$ & 0 . & 0 . & 0 . & 0 . & 0 . & 0 & -50 \\
\hline 3 & 3 & $1032-21$ & 0 . & 0 . & 0 . & 0 . & 0. & 0 & -50 . \\
\hline 3 & 3 & $997-30$ & 500 . & 0 . & 0 . & 0 . & 1824 . & 58 & 0 \\
\hline 5 & 1 & $988-36$ & 0 . & 0 . & 0 . & 0 . & 38 & 13 & 0 . \\
\hline 5 & 1 & $987-38$. & 0 . & 0 . & 0 . & 0 . & 28 & 9 & 0 . \\
\hline 5 & 1 & $997-40$. & 0 . & 0 . & 0 . & 0 . & 101. & 33 & 15. \\
\hline 5 & 1 & $990-37$ & -72 . & 3. & 0 . & 0 . & 0 & 0 & 0 . \\
\hline 5 & 1 & $1004-41$ & 0 & 0 . & 0 . & 0 . & 53. & 15 & 20. \\
\hline 5 & 1 & $998-43$ & 0 . & 0 . & 0 . & 0 . & 45. & 15 & 20. \\
\hline 5 & 1 & $998-43$. & 0 . & 0 . & 0 . & 0 . & 14. & 5 & 3. \\
\hline 5 & 1 & $989-44$. & 0 . & 0 . & 0 . & 0 . & 54. & 15 & 5. \\
\hline 5 & 1 & $995-43$. & 0 . & 0 . & 0 . & 0. & 32. & 9 & 3. \\
\hline 3 & 3 & $986-31$. & 0 . & 0 . & 0 . & 0 . & 0 & 0 & 0 . \\
\hline 5 & 1 & $1018-38$ & 0 . & 0 . & 0 . & 0 . & 15. & 4 & 2 . \\
\hline 3 & 3 & 987-33 & 0 . & 0 . & 0 . & 0 . & 0 . & 0 & 0 . \\
\hline 5 & 1 & $999-42$. & 0 . & 0 . & 0 . & 0 . & 53. & 18 & 0 . \\
\hline 5 & 1 & $1007-40$. & 0 . & 0 . & 0 . & 0 . & 39. & 13 & 0 . \\
\hline 3 & 3 & $1003-33$ & 0 . & 0 & 0 . & 0 . & 0 . & 0 & 0 . \\
\hline 5 & 1 & $1011-36$ & -41 . & 17. & 0 . & 0 . & 0 . & 0 & 0 . \\
\hline 5 & 1 & $1007-36$ & 0 . & 0 & 0 . & 0 . & 16. & 4 & 0 . \\
\hline 5 & 1 & 995-36 & 0 . & 0 . & 0 . & 0 . & 20. & 5 & 2. \\
\hline 5 & 1 & $998-39$. & 0 . & 0 . & 0 . & 0 . & 65. & 22 & 26. \\
\hline 5 & 1 & $990-42$. & 0 . & 0 & 0 . & 0 . & 5. & 2 & 0 . \\
\hline 5 & 1 & $999-41$. & 60. & 30. & 0 . & 0 . & 94. & 23 & 0 . \\
\hline 5 & 1 & $988-42$. & 0 . & 0 & 0 . & 0 . & 7. & 2 & 0 . \\
\hline 2 & 1 & $996-39$. & 100. & 23. & 0 . & 0 . & 0 . & 0 & 0 . \\
\hline 5 & 1 & $991-38$. & 0 . & 0 & 0 . & 0 . & 0 . & 0 & 0 . \\
\hline 5 & 1 & $1014-39$ & 0 . & 0 . & 0 . & 0 . & 218 . & 43 & 0 . \\
\hline 5 & 1 & 995-31 & 0 . & 0 . & 0 . & 0 . & 0 & 0 & 0 . \\
\hline 5 & 1 & $988-38$ & 24 . & 9. & 0 . & 0 . & 94. & 30 & 0 . \\
\hline 5 & 1 & $995-30$. & 0 . & 0 . & 0 . & 0 . & 0 . & 0 & 0 . \\
\hline 5 & 1 & $991-40$. & 0 . & 0 & 0 . & 0 . & 25. & 7 & 3. \\
\hline 5 & 1 & 997-38. & 0 . & 20 . & 0 . & 0 . & 48. & 16 & 0 . \\
\hline 4 & 2 & $1008-29$ & 0 . & 0 & 0 . & 0 . & 0 . & 0 & 0 . \\
\hline 4 & 2 & $984-33$ & 0 . & 0 & 0 . & 0 . & 84. & 27 & 0 . \\
\hline 7 & 1 & $992-34$. & 244. & -26 & 0 . & 0 . & 0 . & 0 & 0 . \\
\hline 4 & 2 & 983-31 & 0 . & 0 & 0 . & 0 . & 0 . & 0 & 0 . \\
\hline 4 & 2 & $1023-18$ & 866. & 71. & 0 . & 0 . & 0 . & 0 & 0 . \\
\hline 4 & 2 & $988-30$ & -59. & 3. & 0 . & 0 . & 0 . & 0 & 0 . \\
\hline & 3 & $1015-29$ & 0 . & 0 & 0 . & 0 . & 0 . & 0 & 0 . \\
\hline 3 & 3 & $996-25$ & 26. & -116. & 0 . & 0 . & 0 . & 0 & 0 \\
\hline 3 & 3 & $1000-31$ & 0 . & 0 & 0 . & 0 . & 0 . & 0 & -50 \\
\hline 5 & 1 & 999-42. & 0 . & 0 . & 0 . & 0 . & 23. & 7 & 6 \\
\hline 5 & 1 & $1013-40$. & 0 . & 0 . & 0 . & 0 . & 40. & 13 & 12. \\
\hline 5 & 1 & $1020-39$ & 0 . & 0 . & 0 . & 0 . & 0 . & 0 & 0 . \\
\hline 5 & 1 & $1026-24$ & 0 . & 0 . & 0 . & 0 . & 0 & 0 & 0 \\
\hline 5 & 1 & 992-32. & 0 . & 0 & 0 . & 0 . & 30 . & 12 & 12. \\
\hline 5 & 1 & $1016-31$ & -427. & -110 & 0 & 0 & 0 . & 0 & 0 \\
\hline G & & $1000-18$ & 447. & 86. & -72. & 267 . & 0 . & 0 & 0 . \\
\hline 3 & 3 & $1011-34$ & 0 & 0 & 0 . & 0 & 0 . & 0 & 0 \\
\hline 3 & 3 & $1029-25$ & 0 & 0 & 0 & 0 . & 0 . & 0 & -50 . \\
\hline G & & $1000-16.1$ & 055. & 136. & 170 . & 605. & 0 . & 0 & 0 \\
\hline 5 & 1 & $1011-40$ & 0 . & 0 & 0 . & 0 & 26. & 9 & 5. \\
\hline 5 & 1 & $1022-38$ & 0 . & 0 . & 0 . & 0 . & 79. & 0 & 0 . \\
\hline 5 & 1 & $1031-36$. & 0 . & 0 . & 0 . & 0 . & 0 . & 0 & 0 . \\
\hline 5 & 1 & $1009-38$. & 0 . & 0 . & 0 . & 0 . & 16. & 5 & 3. \\
\hline 8 & & $1000-46$ & 0 . & 0 . & 0 . & 0 . & 51. & 17 & 22 . \\
\hline 5 & 1 & $998-42$ & 0 . & 0 . & 0 . & 0 . & 62. & 20 & 10. \\
\hline $\mathrm{T}$ & 1 & $1003-41$. & 0 . & 0 . & 0 . & 0 . & 0 . & 0 & 0 \\
\hline 5 & 1 & $1009-38$. & 0 . & 0 . & 0 . & 0 . & 38 . & 9 & 3. \\
\hline 5 & 1 & $970-41$. & 0 . & 0. & 0 . & 0 . & 41. & 11 & 12. \\
\hline 5 & 1 & $969-45$. & 0 . & 0 . & 0 . & 0 . & 51. & 13 & 12 . \\
\hline 7 & 1 & $979-44$. & 0 . & 0 . & 0 . & 0 . & 17. & 4 & 0 . \\
\hline 8 & & $988-45$. & 0 . & 0 . & 0 . & 0 . & 61. & 4 & 0 . \\
\hline 7 & 1 & $990-46$. & 0 . & 0 . & 0. & 0 . & 0 . & 0 & \\
\hline
\end{tabular}




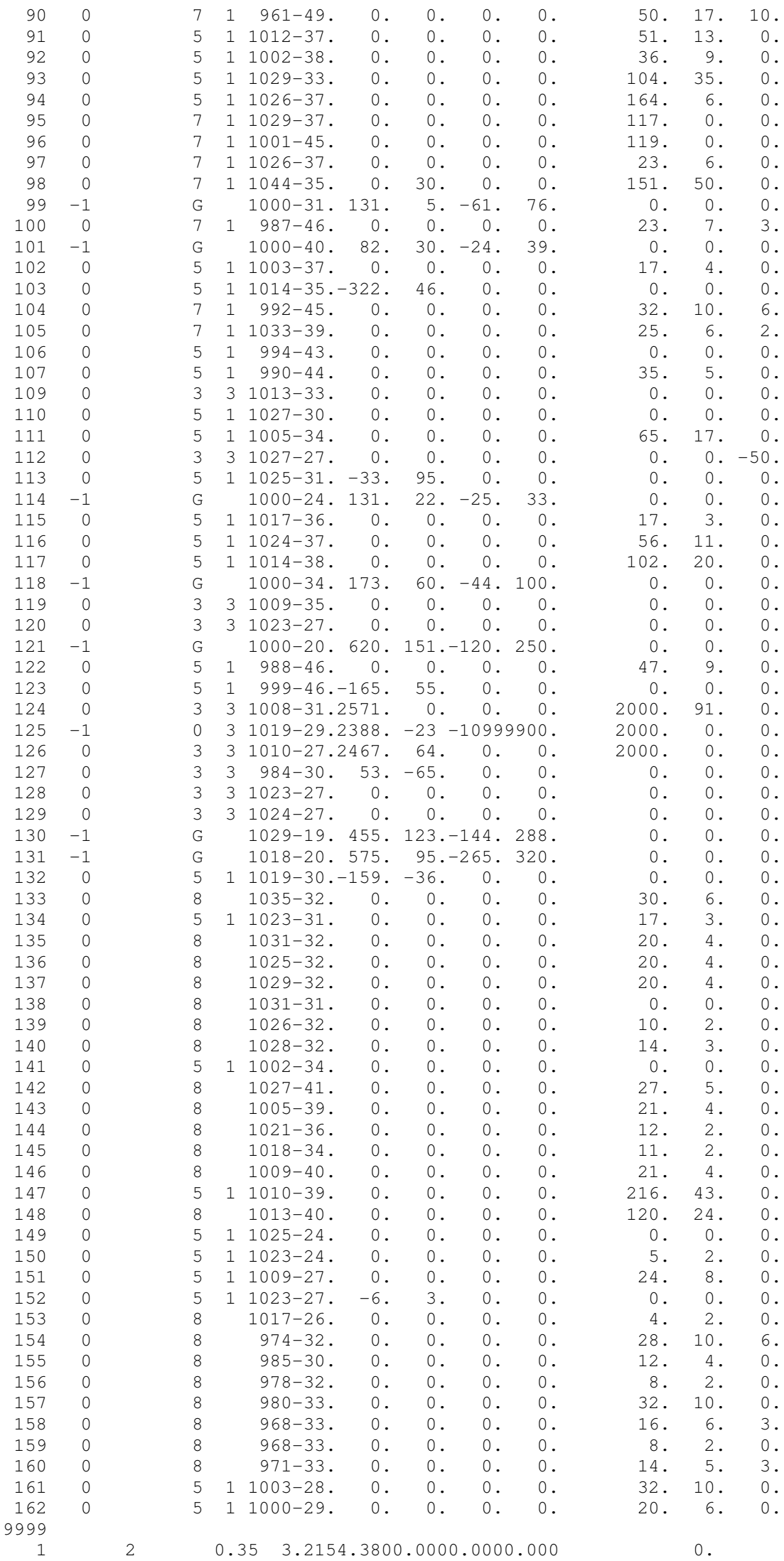




\begin{tabular}{|c|c|c|}
\hline 3 & 3.2672 .2400 .0000 .0000 .000 & 0 . \\
\hline 4 & 6.2198 .7000 .0000 .0000 .000 & 0 . \\
\hline 5 & 1.1920 .1200 .0000 .0000 .000 & 0 . \\
\hline 6 & 1.330 .0000 .0000 .0000 .000 & 0 . \\
\hline 7 & 1.2521 .2200 .0000 .0000 .000 & 0 . \\
\hline 13 & $0.46 \quad 4.1770 .5800 .0000 .0000 .000$ & 0 . \\
\hline 14 & 23.61101 .220 .0000 .0000 .0000 .000 & 0 . \\
\hline 50 & 3.8916 .990 .0000 .0000 .0000 .000 & 0 . \\
\hline 103 & 10.74180 .230 .0000 .0000 .0000 .000 & 0 . \\
\hline 123 & 28.83167 .190 .0000 .0000 .0000 .000 & 0 . \\
\hline 124 & 1.4064 .830 .0000 .0000 .0000 .000 & 0 . \\
\hline 125 & 0.8411 .390 .0000 .0000 .0000 .000 & 0 . \\
\hline 112 & $0.59 \quad 5.6892 .5000 .0000 .0000 .000$ & 0 . \\
\hline 115 & 1.850 .0000 .0000 .0000 .000 & 0 . \\
\hline 119 & 1.1920 .5000 .0000 .0000 .000 & 0 . \\
\hline 120 & 2.2437 .9200 .0000 .0000 .000 & 0 . \\
\hline 129 & 2.6846 .1200 .0000 .0000 .000 & 0 . \\
\hline 131 & 1.270 .0000 .0000 .0000 .000 & 0 . \\
\hline 8 & 1.890 .0000 .0000 .0000 .000 & 0 . \\
\hline 9 & 0.171 .6928 .7200 .0000 .0000 .000 & 0 . \\
\hline 10 & 45.91107 .030 .0000 .0000 .0000 .000 & 0 . \\
\hline 12 & $1.06 \quad 5.74 \quad 0.0000 .0000 .0000 .000$ & 0 . \\
\hline 13 & $12.74 \quad 47.84 \quad 0.0000 .0000 .0000 .000$ & 0 . \\
\hline 14 & 4.7339 .560 .0000 .0000 .0000 .000 & 0 . \\
\hline 15 & 50.35174 .330 .0000 .0000 .0000 .000 & 0 . \\
\hline 132 & 2.5228 .800 .0000 .0000 .0000 .000 & 0 . \\
\hline 75 & 0.131 .5026 .8200 .0000 .0000 .000 & 0 . \\
\hline 11 & $0.513 .70 \quad 7.1600 .0000 .0000 .000$ & 0 . \\
\hline 13 & 12.9962 .200 .0000 .0000 .0000 .000 & 0 . \\
\hline 15 & 12.7570 .330 .0000 .0000 .0000 .000 & 0 . \\
\hline 60 & 25.25122 .420 .0000 .0000 .0000 .000 & 0 . \\
\hline 15 & 2.8517 .9334 .8400 .0000 .0000 .000 & 0 . \\
\hline 46 & 1.4212 .2518 .7600 .0000 .0000 .000 & 0 . \\
\hline 58 & 1.7010 .7020 .7400 .0000 .0000 .000 & 0 . \\
\hline 59 & 0.714 .718 .5200 .0000 .0000 .000 & 0 . \\
\hline 2 & $0.08 \quad 3.77 \quad 0.0000 .0000 .0000 .000$ & 0 . \\
\hline 13 & 10.3831 .370 .0000 .0000 .0000 .000 & 0 . \\
\hline 14 & 15.9864 .150 .0000 .0000 .0000 .000 & 0 . \\
\hline 132 & 44.86157 .730 .0000 .0000 .0000 .000 & 0 . \\
\hline 15 & $4.40 \quad 32.27 \quad 0.0000 .0000 .0000 .000$ & 0 . \\
\hline 62 & $0.98 \quad 12.210 .0000 .0000 .0000 .000$ & 0 . \\
\hline 72 & $1.078 .28 \quad 0.0000 .0000 .0000 .000$ & 0 . \\
\hline 113 & 3.820 .0000 .0000 .0000 .000 & 0 . \\
\hline 132 & 3.740 .0000 .0000 .0000 .000 & 0 . \\
\hline 58 & 1.157 .3214 .2000 .0000 .0000 .000 & 0 . \\
\hline 60 & 39.07167 .530 .0000 .0000 .0000 .000 & 0 . \\
\hline 62 & $0.84 \quad 5.88 \quad 0.0000 .0000 .0000 .000$ & 0 . \\
\hline 63 & 17.04145 .550 .0000 .0000 .0000 .000 & 0 . \\
\hline 17 & 60.17143 .730 .0000 .0000 .0000 .000 & 0 . \\
\hline 18 & 2.9710 .705 .4600 .0000 .0000 .000 & 0 . \\
\hline 27 & 15.7488 .710 .0000 .0000 .0000 .000 & 0 . \\
\hline 126 & 10.5351 .320 .0000 .0000 .0000 .000 & 0 . \\
\hline 127 & 9.5852 .760 .0000 .0000 .0000 .000 & 0 . \\
\hline 18 & 2.1310 .136 .4200 .0000 .0000 .000 & 0 . \\
\hline 19 & $23.1476 .78 \quad 0.0000 .0000 .0000 .000$ & 0 . \\
\hline 21 & 4.7126 .650 .0000 .0000 .0000 .000 & 0 . \\
\hline 127 & 2.8726 .370 .0000 .0000 .0000 .000 & 0 . \\
\hline 30 & $2.0710 .88 \quad 5.2000 .0000 .0000 .000$ & 0 . \\
\hline 32 & 2.3412 .205 .8200 .0000 .0000 .000 & 0 . \\
\hline 37 & $0.00 \quad 4.56 \quad 0.0000 .0000 .0000 .000$ & 0 . \\
\hline 21 & 38.67190 .050 .0000 .0000 .0000 .000 & 0 . \\
\hline 38 & 2.3912 .505 .9600 .0000 .0000 .000 & 0 . \\
\hline 43 & 6.0325 .720 .0000 .0000 .0000 .000 & 0 . \\
\hline 127 & 10.7468 .090 .0000 .0000 .0000 .000 & 0 . \\
\hline 53 & $0.0011 .40 \quad 0.0000 .0000 .0000 .000$ & 0 . \\
\hline 157 & 1.132 .790 .0400 .0000 .0000 .000 & 0 . \\
\hline 22 & 3.1216 .297 .7800 .0000 .0000 .000 & 0 . \\
\hline 127 & 1.0564 .140 .0000 .0000 .0000 .000 & 0 . \\
\hline 38 & $\begin{array}{lll}1.40 & 5.40 & 2.5000 .0000 .0000 .000\end{array}$ & 0 . \\
\hline 39 & 4.930 .0000 .0000 .0000 .000 & 0 . \\
\hline 40 & 7.173 .2800 .0000 .0000 .000 & 0 . \\
\hline 41 & 8.504 .0400 .0000 .0000 .000 & 0 . \\
\hline 24 & $1.74 \quad 5.112 .3000 .0000 .0000 .000$ & 0 . \\
\hline 60 & 6.6030 .930 .0000 .0000 .0000 .000 & 0 . \\
\hline
\end{tabular}




\begin{tabular}{|c|c|c|c|c|}
\hline 24 & 25 & 0.00 & 3.400 .0000 .0000 .0000 .000 & 0 . \\
\hline 24 & 28 & 2.49 & 7.252 .0200 .0000 .0000 .000 & 0 . \\
\hline 24 & 45 & 1.37 & 7.253 .4000 .0000 .0000 .000 & 0 . \\
\hline 25 & 26 & 0.59 & 5.8393 .0200 .0000 .0000 .000 & 0 . \\
\hline 25 & 27 & 0.44 & 4.1083 .8400 .0000 .0000 .000 & 0 . \\
\hline 26 & 74 & 0.63 & 6.0793 .0000 .0000 .0000 .000 & 0 . \\
\hline 26 & 75 & 0.30 & 3.2250 .3800 .0000 .0000 .000 & 0 . \\
\hline 26 & 76 & 0.00 & 0.820 .0000 .0000 .0000 .000 & 0 . \\
\hline 27 & 31 & 1.011 & 12.730 .0000 .0000 .0000 .000 & 0 . \\
\hline 27 & 62 & 1.735 & 58.100 .0000 .0000 .0000 .000 & 0 . \\
\hline 27 & 65 & 1.052 & 27.640 .0000 .0000 .0000 .000 & 0 . \\
\hline 27 & 125 & 3.5016 & 68.450 .0000 .0000 .0000 .000 & 0 . \\
\hline 27 & 126 & 0.22 & 2.250 .0000 .0000 .0000 .000 & 0 . \\
\hline 27 & 127 & 15.0614 & 43.550 .0000 .0000 .0000 .000 & 0 . \\
\hline 28 & 29 & 2.40 & 9.654 .4400 .0000 .0000 .000 & 0 . \\
\hline 29 & 30 & 3.801 & 15.006 .9600 .0000 .0000 .000 & 0 . \\
\hline 29 & 31 & 2.06 & 8.333 .8400 .0000 .0000 .000 & 0 . \\
\hline 30 & 32 & 2.49 & 10.054 .5800 .0000 .0000 .000 & 0 . \\
\hline 32 & 33 & 1.14 & 4.482 .0800 .0000 .0000 .000 & 0 . \\
\hline 33 & 34 & 2.801 & 11.405 .2000 .0000 .0000 .000 & 0 . \\
\hline 33 & 35 & 2.16 & 10.705 .1000 .0000 .0000 .000 & 0 . \\
\hline 33 & 36 & 1.02 & 5.362 .5400 .0000 .0000 .000 & 0 . \\
\hline 34 & 40 & 3.971 & 15.176 .9000 .0000 .0000 .000 & 0 . \\
\hline 34 & 77 & 2.35 & 8.964 .0800 .0000 .0000 .000 & 0 . \\
\hline 35 & 40 & 2.71 & 13.416 .3800 .0000 .0000 .000 & 0 . \\
\hline 36 & 67 & 1.76 & 9.244 .4000 .0000 .0000 .000 & 0 . \\
\hline 37 & 39 & 0.39 & 3.7967 .0000 .0000 .0000 .000 & 0 . \\
\hline 37 & 126 & 0.40 & 3.8167 .0000 .0000 .0000 .000 & 0 . \\
\hline 37 & 127 & 0.40 & 4.0368 .3200 .0000 .0000 .000 & 0 . \\
\hline 39 & 42 & 0.20 & 1.8632 .0000 .0000 .0000 .000 & 0 . \\
\hline 40 & 81 & 3.003 & 34.500 .3800 .0000 .0000 .000 & 0 . \\
\hline 40 & 82 & 0.40 & 1.901 .0800 .0000 .0000 .000 & 0 . \\
\hline 41 & 81 & 3.703 & 37.200 .5800 .0000 .0000 .000 & 0 . \\
\hline 41 & 83 & 0.52 & 2.561 .2400 .0000 .0000 .000 & 0 . \\
\hline 41 & 84 & 0.57 & 5.802 .9200 .0000 .0000 .000 & 0 . \\
\hline 42 & 109 & 0.19 & 1.9633 .3000 .0000 .0000 .000 & 0 . \\
\hline 43 & 44 & 1.88 & 7.513 .4800 .0000 .0000 .000 & 0 . \\
\hline 43 & 103 & 3.241 & 17.020 .0000 .0000 .0000 .000 & 0 . \\
\hline 43 & 124 & 2.931 & 17.660 .0000 .0000 .0000 .000 & 0 . \\
\hline 43 & 125 & 14.496 & 65.090 .0000 .0000 .0000 .000 & 0 . \\
\hline 44 & 102 & 1.30 & 5.002 .3600 .0000 .0000 .000 & 0 . \\
\hline 44 & 103 & 1.27 & 5.102 .4400 .0000 .0000 .000 & 0 . \\
\hline 45 & 54 & 1.08 & 5.702 .7200 .0000 .0000 .000 & 0 . \\
\hline 46 & 47 & 3.10 & 13.786 .2200 .0000 .0000 .000 & 0 . \\
\hline 47 & 48 & 2.51 & 11.145 .0200 .0000 .0000 .000 & 0 . \\
\hline 47 & 49 & 0.30 & 1.200 .5400 .0000 .0000 .000 & 0 . \\
\hline 48 & 50 & 3.361 & 16.607 .8000 .0000 .0000 .000 & 0 . \\
\hline 48 & 51 & 4.20 & $13.00 \quad 5.7000 .0000 .0000 .000$ & 0 . \\
\hline 48 & 52 & 5.40 & 16.807 .4000 .0000 .0000 .000 & 0 . \\
\hline 49 & 87 & 1.40 & 6.802 .6600 .0000 .0000 .000 & 0 . \\
\hline 50 & 51 & 3.00 & 9.004 .1000 .0000 .0000 .000 & 0 . \\
\hline 50 & 123 & 40.7118 & 85.430 .0000 .0000 .0000 .000 & 0 . \\
\hline 50 & 125 & 13.376 & 60.310 .0000 .0000 .0000 .000 & 0 . \\
\hline 51 & 141 & 3.23 & $10.00 \quad 4.4200 .0000 .0000 .000$ & 0 . \\
\hline 52 & 79 & 6.23 & 21.269 .4000 .0000 .0000 .000 & 0 . \\
\hline 52 & 106 & 2.31 & 7.173 .1400 .0000 .0000 .000 & 0 . \\
\hline 52 & 116 & 0.60 & 4.872 .5600 .0000 .0000 .000 & 0 . \\
\hline 52 & 117 & 1.17 & 4.932 .3000 .0000 .0000 .000 & 0 . \\
\hline 52 & 118 & 0.00 & 5.200 .0000 .0000 .0000 .000 & 0 . \\
\hline 53 & 11 & 0.05 & 2.000 .0000 .0000 .0000 .000 & 0 . \\
\hline 53 & 54 & 2.751 & 19.619 .5600 .0000 .0000 .000 & 0 . \\
\hline 53 & 55 & 0.05 & 0.260 .2200 .0000 .0000 .000 & 0 . \\
\hline 54 & 56 & 1.74 & 9.104 .3000 .0000 .0000 .000 & 0 . \\
\hline 54 & 57 & 2.50 & 12.375 .8800 .0000 .0000 .000 & 0 . \\
\hline 55 & 57 & 4.621 & 17.638 .0200 .0000 .0000 .000 & 0 . \\
\hline 55 & 149 & 1.53 & 6.713 .1200 .0000 .0000 .000 & 0 . \\
\hline 55 & 162 & 0.40 & 1.890 .9800 .0000 .0000 .000 & 0 . \\
\hline 56 & 67 & 1.70 & 8.944 .2400 .0000 .0000 .000 & 0 . \\
\hline 57 & 80 & 2.721 & 10.374 .7200 .0000 .0000 .000 & 0 . \\
\hline 58 & 61 & 1.331 & 10.1818 .4200 .0000 .0000 .000 & 0 . \\
\hline 59 & 61 & 1.06 & 7.0612 .1000 .0000 .0000 .000 & 0 . \\
\hline 60 & 61 & 0.12 & $2.45-0.2200 .0000 .0000 .000$ & 0 . \\
\hline 60 & 62 & 36.749 & 96.400 .0000 .0000 .0000 .000 & 0 . \\
\hline 60 & 65 & $10.41 \leq$ & 41.440 .0000 .0000 .0000 .000 & \\
\hline
\end{tabular}




\begin{tabular}{|c|c|c|c|c|}
\hline 60 & 126 & 53.671 & 82.950 .0000 .0000 .0000 .000 & 0 . \\
\hline 61 & 62 & 2.962 & 22.7539 .9600 .0000 .0000 .000 & 0 . \\
\hline 61 & 63 & 0.43 & 4.227 .6400 .0000 .0000 .000 & 0 . \\
\hline 62 & 63 & 1.58 & 17.020 .0000 .0000 .0000 .000 & 0 . \\
\hline 62 & 65 & 0.40 & 7.400 .0000 .0000 .0000 .000 & 0 . \\
\hline 62 & 126 & 0.442 & 29.690 .0000 .0000 .0000 .000 & 0 . \\
\hline 63 & 65 & 24.091 & 96.000 .0000 .0000 .0000 .000 & 0 . \\
\hline 64 & 65 & 0.50 & 5.7190 .9800 .0000 .0000 .000 & 0 . \\
\hline 64 & 66 & 0.33 & 3.8160 .6600 .0000 .0000 .000 & 0 . \\
\hline 65 & 126 & 0.31 & 15.360 .0000 .0000 .0000 .000 & 0 . \\
\hline 66 & 11 & 0.00 & 1.180 .0000 .0000 .0000 .000 & 0 . \\
\hline 67 & 68 & 1.93 & 10.134 .8200 .0000 .0000 .000 & 0 . \\
\hline 68 & 69 & 0.68 & 3.531 .6800 .0000 .0000 .000 & 0 . \\
\hline 69 & 77 & 0.98 & 3.741 .7000 .0000 .0000 .000 & 0 . \\
\hline 69 & 78 & 1.14 & 4.341 .9600 .0000 .0000 .000 & 0 . \\
\hline 69 & 79 & 0.52 & 4.332 .2000 .0000 .0000 .000 & 0 . \\
\hline 70 & 73 & 0.00 & 1.970 .0000 .0000 .0000 .000 & 0 . \\
\hline 70 & 149 & 0.01 & 0.090 .2000 .0000 .0000 .000 & 0 . \\
\hline 71 & 85 & 3.041 & 15.067 .1600 .0000 .0000 .000 & 0 . \\
\hline 71 & 150 & 1.96 & 9.704 .6200 .0000 .0000 .000 & 0 . \\
\hline 72 & 113 & 0.22 & 1.300 .0000 .0000 .0000 .000 & 0 . \\
\hline 72 & 132 & 0.28 & 1.680 .0000 .0000 .0000 .000 & 0 . \\
\hline 72 & 152 & 3.85 & 18.000 .0000 .0000 .0000 .000 & 0 . \\
\hline 74 & 119 & 0.31 & 3.1048 .2200 .0000 .0000 .000 & 0 . \\
\hline 75 & 128 & 0.08 & 0.8716 .6000 .0000 .0000 .000 & 0 . \\
\hline 75 & 130 & 0.04 & 2.420 .0000 .0000 .0000 .000 & 0 . \\
\hline 78 & 79 & 0.51 & 3.361 .8200 .0000 .0000 .000 & 0 . \\
\hline 78 & 80 & 2.44 & 9.304 .2200 .0000 .0000 .000 & 0 . \\
\hline 79 & 74 & 0.00 & 1.800 .0000 .0000 .0000 .000 & 0 . \\
\hline 82 & 83 & 0.53 & 2.491 .3000 .0000 .0000 .000 & 0 . \\
\hline 84 & 93 & 1.25 & 8.264 .1400 .0000 .0000 .000 & 0 . \\
\hline 85 & 86 & 2.11 & 10.464 .9800 .0000 .0000 .000 & 0 . \\
\hline 86 & 87 & 2.80 & 11.205 .3800 .0000 .0000 .000 & 0 . \\
\hline 86 & 88 & 4.402 & 22.8010 .9000 .0000 .0000 .000 & 0 . \\
\hline 88 & 96 & 7.402 & 25.001 .4200 .0000 .0000 .000 & 0 . \\
\hline 88 & 106 & 0.79 & 4.682 .3200 .0000 .0000 .000 & 0 . \\
\hline 89 & 86 & 0.00 & 5.700 .0000 .0000 .0000 .000 & 0 . \\
\hline 89 & 90 & 6.901 & 13.401 .4000 .0000 .0000 .000 & 0 . \\
\hline 90 & 96 & 18.37 & 35.903 .7000 .0000 .0000 .000 & 0 . \\
\hline 91 & 92 & 1.56 & 8.193 .7600 .0000 .0000 .000 & 0 . \\
\hline 91 & 93 & 1.43 & 8.954 .5000 .0000 .0000 .000 & 0 . \\
\hline 91 & 94 & 1.45 & 9.574 .8000 .0000 .0000 .000 & 0 . \\
\hline 92 & 102 & 1.50 & 6.102 .9200 .0000 .0000 .000 & 0 . \\
\hline 93 & 42 & 0.00 & 2.600 .0000 .0000 .0000 .000 & 0 . \\
\hline 93 & 108 & 0.00 & 1.540 .0000 .0000 .0000 .000 & 0 . \\
\hline 94 & 103 & 2.27 & 13.336 .6000 .0000 .0000 .000 & 0 . \\
\hline 94 & 107 & 6.131 & 18.918 .3600 .0000 .0000 .000 & 0 . \\
\hline 94 & 109 & 0.00 & 3.500 .0000 .0000 .0000 .000 & 0 . \\
\hline 95 & 91 & 0.54 & $4.58-0.3600 .0000 .0000 .000$ & 0 . \\
\hline 95 & 96 & 8.702 & 21.208 .6000 .0000 .0000 .000 & 0 . \\
\hline 95 & 97 & 12.892 & 28.093 .3400 .0000 .0000 .000 & 0 . \\
\hline 95 & 98 & 0.71 & 4.302 .2400 .0000 .0000 .000 & 0 . \\
\hline 95 & 99 & 0.00 & 6.850 .0000 .0000 .0000 .000 & 0 . \\
\hline 96 & 100 & 6.90 & 16.101 .8600 .0000 .0000 .000 & 0 . \\
\hline 96 & 101 & 0.001 & 10.310 .0000 .0000 .0000 .000 & 0 . \\
\hline 97 & 44 & 0.51 & $10.07-0.2500 .0000 .0000 .000$ & 0 . \\
\hline 98 & 93 & 0.06 & $2.14-3.4100 .0000 .0000 .000$ & 0 . \\
\hline 98 & 105 & 14.852 & 29.303 .1000 .0000 .0000 .000 & 0 . \\
\hline 100 & 104 & 6.20 & 14.501 .6600 .0000 .0000 .000 & 0 . \\
\hline 103 & 123 & 18.20 & 75.100 .0000 .0000 .0000 .000 & 0 . \\
\hline 103 & 124 & 0.02 & 1.670 .0000 .0000 .0000 .000 & 0 . \\
\hline 103 & 125 & 2.791 & 19.720 .0000 .0000 .0000 .000 & 0 . \\
\hline 104 & 34 & 0.80 & $6.37-0.3300 .0000 .0000 .000$ & 0 . \\
\hline 105 & 38 & 0.00 & 11.600 .0000 .0000 .0000 .000 & 0 . \\
\hline 106 & 107 & 1.96 & 6.112 .6800 .0000 .0000 .000 & 0 . \\
\hline 107 & 122 & 1.30 & 6.212 .9600 .0000 .0000 .000 & 0 . \\
\hline 109 & 119 & 0.60 & 5.7792 .9000 .0000 .0000 .000 & 0 . \\
\hline 109 & 124 & 0.20 & 2.2237 .8200 .0000 .0000 .000 & 0 . \\
\hline 109 & 125 & 0.70 & 6.20100 .000 .0000 .0000 .000 & 0 . \\
\hline 110 & 111 & 2.30 & 9.904 .6000 .0000 .0000 .000 & 0 . \\
\hline 110 & 112 & 0.00 & 1.850 .0000 .0000 .0000 .000 & 0 . \\
\hline 110 & 114 & 0.00 & 7.680 .0000 .0000 .0000 .000 & 0 . \\
\hline 110 & 134 & 0.32 & 2.561 .3400 .0000 .0000 .000 & 0 . \\
\hline 110 & 141 & 2.10 & 6.492 .8800 .0000 .0000 .000 & \\
\hline
\end{tabular}




\begin{tabular}{|c|c|c|c|c|}
\hline 111 & 115 & 5.27 & 22.151 & 0.3000 .0000 .0000 .000 \\
\hline 112 & 120 & 0.05 & 0.44 & 7.2000 .0000 .0000 .000 \\
\hline 112 & 121 & 0.00 & 1.90 & 0.0000 .0000 .0000 .000 \\
\hline 113 & 132 & 4.59 & 29.11 & 0.0000 .0000 .0000 .000 \\
\hline 113 & 134 & 0.08 & 0.72 & 0.3800 .0000 .0000 .000 \\
\hline 115 & 117 & 0.19 & 1.54 & 3.3000 .0000 .0000 .000 \\
\hline 116 & 117 & 0.48 & 3.91 & 2.1400 .0000 .0000 .000 \\
\hline 116 & 119 & 0.00 & 0.90 & 0.0000 .0000 .0000 .000 \\
\hline 116 & 147 & 0.35 & 2.86 & 1.5600 .0000 .0000 .000 \\
\hline 117 & 147 & 0.22 & 1.75 & 1.0000 .0000 .0000 .000 \\
\hline 120 & 14 & 0.03 & 1.88 & 0.0000 .0000 .0000 .000 \\
\hline 120 & 128 & 0.04 & 0.511 & 0.0000 .0000 .0000 .000 \\
\hline 120 & 129 & 0.03 & 0.38 & 6.5200 .0000 .0000 .000 \\
\hline 122 & 123 & 1.75 & 8.35 & 3.9800 .0000 .0000 .000 \\
\hline 123 & 125 & 4.23 & 24.41 & 0.0000 .0000 .0000 .000 \\
\hline 124 & 125 & 1.13 & 15.85 & 0.0000 .0000 .0000 .000 \\
\hline 124 & 126 & 5.77 & 82.56 & 0.0000 .0000 .0000 .000 \\
\hline 125 & 126 & 2.01 & 59.15 & 0.0000 .0000 .0000 .000 \\
\hline 126 & 127 & 8.77 & 70.49 & 0.0000 .0000 .0000 .000 \\
\hline 128 & 72 & 0.04 & 1.80 & 0.0000 .0000 .0000 .000 \\
\hline 129 & 132 & 0.04 & 1.98 & 0.0000 .0000 .0000 .000 \\
\hline 133 & 134 & 0.00 & 4.10 & 0.0000 .0000 .0000 .000 \\
\hline 133 & 135 & 1.09 & 2.59 & 0.0400 .0000 .0000 .000 \\
\hline 133 & 136 & 3.90 & 9.90 & 0.1600 .0000 .0000 .000 \\
\hline 133 & 137 & 1.34 & 5.04 & 0.1000 .0000 .0000 .000 \\
\hline 135 & 138 & 4.66 & 11.82 & 0.2000 .0000 .0000 .000 \\
\hline 136 & 139 & 2.60 & 6.50 & 0.1000 .0000 .0000 .000 \\
\hline 137 & 140 & 0.41 & 1.56 & 0.0400 .0000 .0000 .000 \\
\hline 138 & 110 & 0.00 & 4.10 & 0.0000 .0000 .0000 .000 \\
\hline 138 & 139 & 2.60 & 6.50 & 0.1000 .0000 .0000 .000 \\
\hline 138 & 140 & 2.51 & 9.41 & 0.1800 .0000 .0000 .000 \\
\hline 138 & 145 & 9.23 & 23.38 & 0.3800 .0000 .0000 .000 \\
\hline 142 & 51 & 0.00 & 17.28 & 0.0000 .0000 .0000 .000 \\
\hline 142 & 143 & 15.82 & 39.19 & 0.6800 .0000 .0000 .000 \\
\hline 142 & 146 & 16.18 & 38.61 & 0.7000 .0000 .0000 .000 \\
\hline 143 & 144 & 9.27 & 23.22 & 0.2000 .0000 .0000 .000 \\
\hline 144 & 141 & 0.00 & 8.20 & 0.0000 .0000 .0000 .000 \\
\hline 144 & 145 & 8.90 & 22.10 & 0.3200 .0000 .0000 .000 \\
\hline 144 & 146 & 6.80 & 29.06 & 0.5800 .0000 .0000 .000 \\
\hline 148 & 116 & 0.00 & 4.10 & 0.0000 .0000 .0000 .000 \\
\hline 149 & 26 & 0.00 & 1.93 & 0.0000 .0000 .0000 .000 \\
\hline 149 & 150 & 0.10 & 0.85 & 0.2000 .0000 .0000 .000 \\
\hline 149 & 151 & 0.39 & 2.62 & 1.3800 .0000 .0000 .000 \\
\hline 149 & 152 & 2.53 & 11.68 & 5.4400 .0000 .0000 .000 \\
\hline 151 & 161 & 0.21 & 1.38 & 0.7400 .0000 .0000 .000 \\
\hline 153 & 70 & 0.00 & 4.58 & 0.0000 .0000 .0000 .000 \\
\hline 153 & 154 & 7.10 & 28.41 & 0.5400 .0000 .0000 .000 \\
\hline 153 & 155 & 4.30 & 18.56 & 0.3800 .0000 .0000 .000 \\
\hline 154 & 156 & 1.55 & 3.79 & 0.0800 .0000 .0000 .000 \\
\hline 154 & 160 & 1.02 & 4.29 & 0.1000 .0000 .0000 .000 \\
\hline 155 & 156 & 1.76 & 8.22 & 0.1400 .0000 .0000 .000 \\
\hline 156 & 157 & 5.30 & 12.73 & 0.2200 .0000 .0000 .000 \\
\hline 157 & 55 & 0.00 & 8.27 & 0.0000 .0000 .0000 .000 \\
\hline 157 & 158 & 4.89 & 14.04 & 0.2800 .0000 .0000 .000 \\
\hline 158 & 159 & 3.39 & 6.64 & 0.1200 .0000 .0000 .000 \\
\hline 159 & 160 & 1.90 & 8.11 & 1.2000 .0000 .0000 .000 \\
\hline 161 & 162 & 0.22 & 1.03 & 0.5400 .0000 .0000 .000 \\
\hline \multirow[t]{2}{*}{9999} & & & & \\
\hline & & 1.10 & & \\
\hline
\end{tabular}

0.

0.

0.

0.

0.

0.

0.

0.

0.

0.

0.

0 .

0.

0.

0.

0.

0.

0

0.

0.

0.

0.

0.

0.

0.

0.

0.

0 .

0.

0.

0.

0.

0.

0.

0.

0.

0.

0.

\section{A.2.7 BANCO DE DADOS DO SISTEMA IEEE 300 BARRAS}

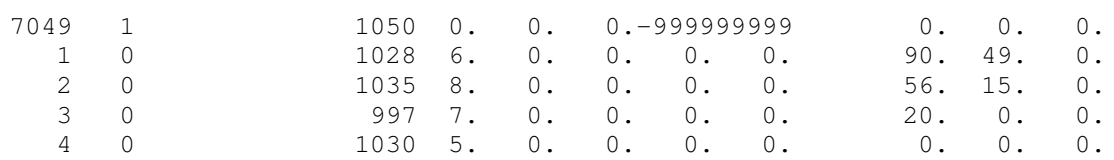




\begin{tabular}{|c|c|c|c|c|c|c|}
\hline 1019 & 5. & 0 . & 0. & 0 . & 353. & 130. \\
\hline 1031 & 7 . & 0 . & 0 . & 0 . & 120. & 41. \\
\hline 993 & 6 . & 0 . & 0. & 0 . & 0 & 0 . \\
\hline 1015 & 2 . & -5 . & $0 .-250$. & 250 . & 58. & 14. \\
\hline 1003 & 3. & 0 & $0 . \quad 0$. & 0 & 96. & 43. \\
\hline 1020 & 1. & -5 & 0. -60 . & 60 . & 148. & 33. \\
\hline 1005 & 2 . & 0 & $0 . \quad 0$. & 0 . & 83. & 21. \\
\hline 997 & 5. & 0 . & 0 . & 0 . & 0 . & 0 . \\
\hline 997 & -1 & 0 . & 0. & 0 . & 58. & 10 . \\
\hline 999 & -5 . & 0 . & 0 . & 0 . & 160. & 60 . \\
\hline 1034 & -9. & 0 . & 0. & 0 . & 127. & 23. \\
\hline 1031 & -3 & 0 . & 0 . & 0 . & 0 & 0 . \\
\hline $1064-$ & 13. & 0 . & 0. & 0 . & 561. & 220. \\
\hline 981 & 1. & 0 . & 0. & 0 . & 0 & 0. \\
\hline 1001 & -2 & -10 & $0 .-200$. & 200. & 595. & 120. \\
\hline 975 & 2. & 0 & $0 . \quad 0$. & 0 & 77. & 1. \\
\hline 996 & -2 . & 0 . & 0. & 0 . & 81. & 23. \\
\hline 1050 & 4. & 0 . & 0 . & 0 . & 21 . & 7. \\
\hline 1005 & 6 . & 0 . & 0 . & 0 . & 0 & 0 . \\
\hline 1023 & 1. & 0 . & 0. & 0 . & 45. & 12. \\
\hline 998 & -2 . & 0 . & 0. & 0 . & 28 . & 9. \\
\hline 975 & -5 & 0 . & 0. & 0 . & 69. & 13. \\
\hline $1024-$ & 12. & 0 . & 0 . & 0 . & 55. & 6. \\
\hline 1041 & -8 & 0 . & 0. & 0 . & 0 & 0 . \\
\hline $975-$ & 26 & 0 . & 0 . & 0 . & 0 . & 0 . \\
\hline $1001-$ & 23. & 0 . & 0. & 0 . & 0. & 0 . \\
\hline $1020-$ & 11. & 0 . & 0 . & 0 . & 85 . & 32 . \\
\hline $1020-$ & 13. & 0 . & 0 . & 0 . & 155. & 18. \\
\hline 1053 & -6 & 0 . & 0. & 0 . & 0. & 0 . \\
\hline $1021-$ & 13. & 0 . & 0 . & 0 . & 46. & -21 . \\
\hline $1029-$ & 10 & 0 . & 0. & 0 . & 86. & 0. \\
\hline 1044 & -7 . & 0 . & 0. & 0 . & 0 . & 0 . \\
\hline $1000-$ & 17. & 0 . & 0 . & 0 . & 39. & 9. \\
\hline $1008-$ & 17. & 0 . & 0 . & 0 . & 195. & 29. \\
\hline $1021-$ & 15. & 0 . & 0 . & 0 . & 0 & 0 . \\
\hline $1034-$ & 12. & 0 . & 0 . & 0 . & 0 . & 0 . \\
\hline $977-$ & 23. & 0 . & 0. & 0 . & 58. & 12. \\
\hline $1001-$ & 16. & 0 . & 0 . & 0 . & 41. & 19. \\
\hline 1047 & -3 & 0 . & 0 . & 0 . & 92. & 26. \\
\hline 1025 & -8 & 0 . & 0. & 0 . & -5 . & 5. \\
\hline $997-$ & 12. & 0 . & 0 . & 0 . & 61. & 28. \\
\hline $995-$ & 18. & 0. & 0. & 0 . & 69. & 3. \\
\hline $1004-$ & 16. & 0. & 0. & 0 . & 10. & 1. \\
\hline $1014-$ & 12. & 0 . & 0 . & 0 . & 22 . & 10. \\
\hline 1033 & -8 & 0 . & 0 . & 0 . & 98. & 20. \\
\hline 991 & -6 & 0 . & 0 . & 0 . & 14. & 1. \\
\hline 978 & -5 & 0 . & 0 . & 0 . & 218 . & 106. \\
\hline $1024-$ & 10 & 0 . & 0 . & 0 . & 0 & 0 \\
\hline 990 & -3 & 0 . & 0 . & 0 . & 227. & 110. \\
\hline 1016 & -1 & 0 . & $0 . \quad 0$. & 0 . & 0 & 0 \\
\hline $958-$ & 18. & 0 . & 0. -55 . & 55. & 70 . & 30 . \\
\hline $948-$ & 13. & 0 . & $0 . \quad 0$. & 0 . & 0 & 0 . \\
\hline $962-$ & 26 & 0 . & 0. & 0 . & 0 . & 0 . \\
\hline $951-$ & 35. & 0 . & 0 . & 0 . & 56. & 20. \\
\hline $979-$ & 30 & 0 . & 0 . & 0 . & 116. & 38. \\
\hline $969-1$ & 27 & 0 . & 0. & 0. & 57. & 19. \\
\hline $977-$ & 26 . & 0 . & 0 . & 0 . & 224 . & 71. \\
\hline $996-$ & 22 & 0 . & $0 . \quad 0$. & 0 . & 0 & 0 \\
\hline $963-2$ & 27 & 0 . & $0 .-212$. & 235. & 208 . & 107. \\
\hline $983-1$ & 25 . & 0 . & $0 . \quad 0$. & 0 & 74 . & 28 \\
\hline $990-$ & 24 . & 0 . & 0 . & 0 . & 0 & 0 . \\
\hline $981-$ & 25. & 0 . & 0. & 0 . & 48. & 14. \\
\hline $987-$ & 25. & 0 . & 0 . & 0 . & 28 . & 7. \\
\hline $1034-$ & 19. & 0 & $0 . \quad 0$. & 0 & 0 & 0 . \\
\hline $1024-$ & 17. & 375 . & $0 .-240$. & 240. & 37. & 13. \\
\hline $987-$ & 18. & 0 & $0 . \quad 0$. & 0 & 0 . & 0 . \\
\hline $990-$ & 14. & 0 . & 0. & 0 . & 0 . & 0 . \\
\hline 992 & -8 & 0 . & 0. & 0 . & 0 . & 0 . \\
\hline $1015-$ & 21 . & 0 . & 0 . & 0 . & 0. & 0 . \\
\hline $1031-$ & 11. & 0 . & 0. & 0 . & 44 . & 0 . \\
\hline $1027-$ & 11. & 0 & 0 & 0 . & 66. & 0 . \\
\hline 1052 & -9 & 155. & $0 .-11$. & 96. & 17. & 0 . \\
\hline 1052 & -6 & 290. & $0 .-153$. & 153. & 16. & 0 . \\
\hline & -9 & & & & 60 . & \\
\hline
\end{tabular}




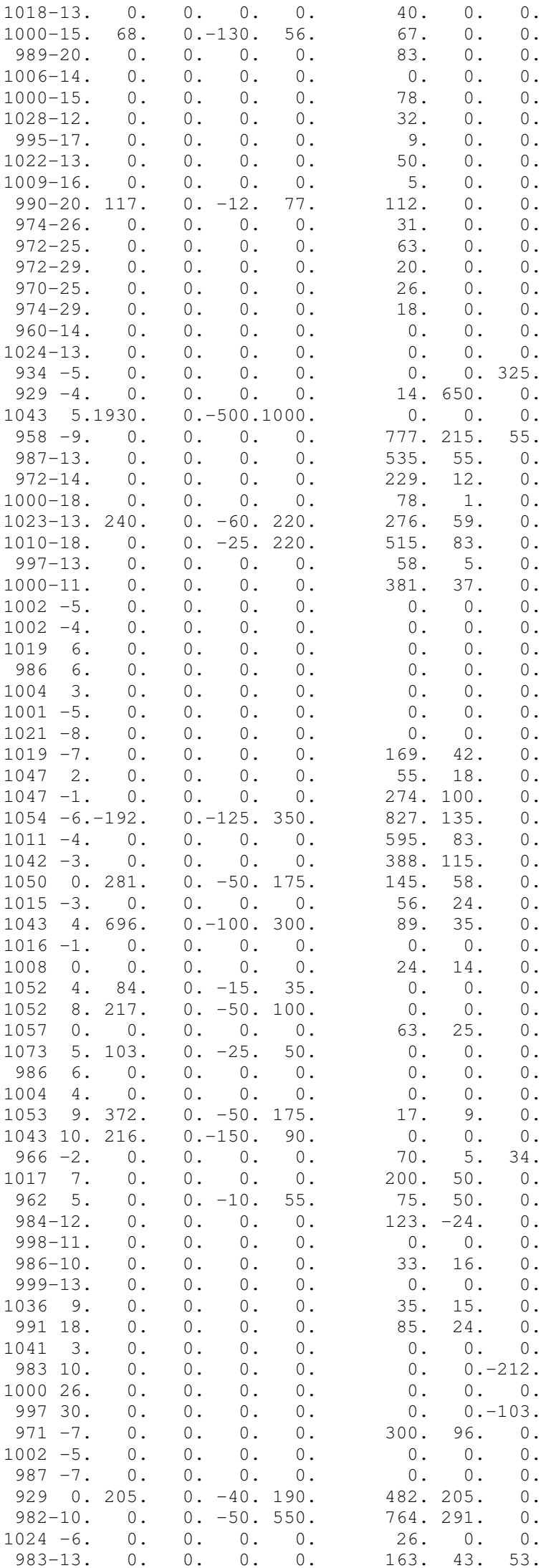




\begin{tabular}{|c|c|c|c|c|c|}
\hline 1062 & -3 & 0 . & 0 . & 0 & 0 . \\
\hline 972 & -7 . & 0 . & 0 . & 176. & 83. \\
\hline 1052 & 228 . & $0 .-45$. & 90 & 5. & 4. \\
\hline 1007 & 84. & $0 .-115$. & 135. & 28. & 12 . \\
\hline 939 & -7 & $0 . \quad 0$. & 0 . & 427 . & 174 . \\
\hline 969 & -9 & 0 . & 0. & 74. & 29. \\
\hline 979 & -3 & 0 . & 0 . & 69. & 49. \\
\hline 1051 & -1 & 0 . & 0 . & 73. & 0 . \\
\hline 1044 & -4 & 0 . & 0 . & 241 . & 89. \\
\hline 971 & 7. & 0 . & 0 . & 40 & 4. \\
\hline 1038 & -7 & $0 . \quad 0$. & 0 . & 137. & 17. \\
\hline 1052 & -4.200 & $0 .-50$. & 80. & 0 & 0 . \\
\hline 1065 & 2.1200 & $0 .-100$. & 400 . & 60. & 24 . \\
\hline 1065 & 1.1200 & $0 .-100$. & 400 . & 60. & 24. \\
\hline 1053 & $-1 . \quad 0$. & $0 . \quad 0$. & 0 & 183. & 44. \\
\hline $997-$ & -26 & $0 . \quad 0$. & 0 . & 7. & 2 \\
\hline $1055-$ & -21.475. & $0 .-400$. & 300 . & 0 & $0 .-150$ \\
\hline 1043 & 12.1973. & $0 .-1000$ & 1000. & 489. & 53. 0. \\
\hline $937-$ & $\begin{array}{ll} & 11 . \\
0\end{array}$ & $0 . \quad 0$. & 0 & 800 . & 72 . \\
\hline $989-$ & -26. & 0 . & 0 . & 0 . & 0 . \\
\hline $1048-$ & -19. & 0 . & 0 . & 0 . & 0 . \\
\hline $1035-$ & -21. & 0 . & 0 . & 0 . & 0 . \\
\hline $969-$ & -25. & 0 . & 0 . & 10. & 3. \\
\hline $990-$ & -24 & 0. & 0 . & 43. & 14. \\
\hline $1014-$ & -21.424 . & $0 .-260$. & 260 . & 64. & 21. \\
\hline $952-$ & -26. & $0 . \quad 0$. & 0 . & 35. & 12. \\
\hline $954-$ & -26. & 0 . & 0 . & 27. & 12. \\
\hline $969-$ & -27. & 0 . & 0 . & 41. & 14. \\
\hline $990-$ & -25. & 0 . & 0 . & 38. & 13. \\
\hline $1003-$ & -22 . & 0 . & 0 . & 42. & 14. \\
\hline $971-$ & -26. & 0 . & 0 . & 72. & 24. \\
\hline $983-$ & -26. & 0 . & 0 . & 0 . & -5 \\
\hline 999 & -27 . & 0 . & 0 . & 12. & 2 \\
\hline $1013-$ & -27 . & 0 . & 0 . & -21 . & -14 . \\
\hline $992-$ & -26. & 0 . & 0 . & 7. & 2 \\
\hline $999-$ & -26. & 0 . & 0 . & 38. & 13. \\
\hline $978-$ & -24. & 0 . & 0 . & 0 . & 0 . \\
\hline $1001-$ & -23. & 0 . & 0 . & 96. & 0 . \\
\hline $1013-$ & -23. 0 . & $0 . \quad 0$. & 0 & 0 & 0 . \\
\hline $1009-$ & -12.272 . & $0 .-150$. & 150 . & 0 & 0 . \\
\hline $991-$ & -18. & $0 . \quad 0$. & 0 & 22. & 16. \\
\hline $986-$ & -20 . & 0 . & 0 . & 47. & 26. \\
\hline $975-$ & -23. & 0 . & 0 . & 176. & 105. \\
\hline $1021-$ & -22 . & 0 . & 0 . & 100. & 75 \\
\hline $1007-$ & -23. & 0 . & 0 . & 131. & 96. \\
\hline $1055-$ & -21. 0. & $0 . \quad 0$. & 0 & 0 & 0 \\
\hline $1008-$ & -22.100. & $0 .-60$. & 560. & 285. & 100. \\
\hline $1000-$ & -22.450. & $0 .-320$. & 320 . & 171. & 70 \\
\hline $1049-$ & -23.250 . & $0 .-300$. & 300 . & 328. & 188. \\
\hline $996-$ & -23. & $0 . \quad 0$. & 0 & 428. & 232 . \\
\hline $1000-$ & -22 . & 0 . & 0 . & 173. & 99. \\
\hline $945-$ & -11. & 0 . & 0 . & 410 . & 40. \\
\hline $1018-$ & -22 . & $0 . \quad 0$. & 0 & 0 & 0 \\
\hline $1000-$ & 303 . & $0 .-300$. & 300 . & 538. & 369. \\
\hline $1042-$ & -21. & $0 . \quad 0$. & 0 . & 223. & 148 . \\
\hline $1049-$ & $-20 . \quad 0$. & $0 . \quad 0$. & 0 . & 96. & 46 \\
\hline $1039-$ & -14.345 . & $0 .-250$. & 250 . & 0 . & 0. \\
\hline $1053-$ & -21. 0. & $0 . \quad 0$. & 0 & 159. & $107 .-300$. \\
\hline $1041-$ & -23. & $0 . \quad 0$. & 0 & 448 & 143. \\
\hline $1000-$ & -26.300 . & $0 .-500$. & 500 . & 404 . & 212 . \\
\hline $1038-$ & -21. & $0 . \quad 0$. & 0 & 572 . & 244 . \\
\hline $1009-$ & -21. & $0 . \quad 0$. & 0 & 269. & 157. \\
\hline $1016-$ & 600 . & $0 .-300$. & 300 . & 0 & 0 \\
\hline $1055-$ & -21. & $0 . \quad 0$. & 0 . & 0. & $0 . \quad 0$ \\
\hline $1009-$ & 250 . & $0 .-200$. & 500 . & 255. & $149 .-150$ \\
\hline $1000-$ & 550 . & $0 .-400$. & 400 . & 0 & $0 . \quad 0$ \\
\hline $1023-$ & -20 . & $0 . \quad 0$. & 0 & 0 . & $0 .-140$. \\
\hline $1049-$ & -16.575. & $0 .-600$. & 600 . & 0 . & $0 . \quad 0$. \\
\hline $992-$ & 170 . & $0 .-40$. & 200 . & 0 . & 0 . \\
\hline $1009-$ & -19. & $0 .-40$. & 80 & 8 . & 3 . \\
\hline $992-$ & -20 . & $0 . \quad 0$. & 0 . & 0 & 0 \\
\hline $971-$ & -21. & 0 . & 0 . & 61. & 30 . \\
\hline $965-$ & -22. & 0. & 0 . & 77. & 33. \\
\hline $968-$ & -22. & $0 . \quad 0$. & 0 . & 61. & 30 \\
\hline
\end{tabular}




\begin{tabular}{|c|c|c|c|c|c|}
\hline $976-25$. & 0 . & 0. & 0 . & 29. & 14. \\
\hline $975-26$ & 0 . & 0 . & 0 . & 29. & 14. \\
\hline $1019-24$. & 0 . & 0 . & 0 . & -23 . & -17 \\
\hline $1025-20$. & 0 . & 0 . & 0 . & -33. & -29. \\
\hline 10151. & 0 . & 0 . & 0 . & 116. & -24 \\
\hline $1014-2$. & 0 . & 0 . & 0 . & 2. & -13. \\
\hline $1000-18$. & 0 . & 0 . & 0 . & 2. & -4 \\
\hline $981-14$ & 0 . & 0 . & 0 . & -15. & 26. \\
\hline $975-23$. & 0 . & 0 . & 0 . & 25 & -1 \\
\hline $942-34$. & 0 . & 0 . & 0 . & 145. & -35 \\
\hline $972-38$ & 0. & 0 . & 0 . & 28. & -20 \\
\hline $960-29$. & 0 . & 0 . & 0 . & 14. & 2. \\
\hline $1000-23$. & 0. & 0 . & 0 . & -11 & -1 \\
\hline $977-28$ & 0 . & 0 . & 0 . & 50. & 17. \\
\hline $958-29$ & 0 . & 0 . & 0 . & 30 & 1. \\
\hline $1030-17$. & 0 . & 0 . & 0 . & -114 . & 77. \\
\hline 10124 . & 0 . & 0 . & 0 . & 100 & 29. \\
\hline $1024-8$. & 0 . & 0 . & 0 . & -100 . & 34. \\
\hline $1012-15$. & 0 . & 0 . & 0 . & 0 & 0. \\
\hline $965-15$ & 0 . & 0. & 0 . & 0. & 0 . \\
\hline 105011. & 467 . & $0 .-210$. & 280. & 0 . & 0 . \\
\hline 105012. & 623. & $0 .-280$. & 280. & 0 . & 0 . \\
\hline 103214. & 210 . & $0 .-420$. & 520. & 0. & 0 . \\
\hline 10145. & 234 . & $0 .-100$. & 100. & 0 . & 0 . \\
\hline 105012. & 372 . & $0 .-224$. & 224 . & 0. & 0 . \\
\hline $1050-10$. & 330 . & $0 . \quad 0$. & 550. & 0 . & 0 . \\
\hline 10506. & 185. & $0 . \quad 0$. & 120. & 0. & 0 . \\
\hline 102913. & 410. & $0 .-224$. & 224 . & 0 . & 0 . \\
\hline 10492 . & 500 . & $0 .-200$. & 200. & 0 . & 0 . \\
\hline 1014-14. & 37. & $0 . \quad 0$. & 42. & 0 . & 0 . \\
\hline $996-8$ & 45. & 0. & 125. & 0. & 0 . \\
\hline $1021-3$. & 165. & 0. -90 . & 90. & 0 . & 0 . \\
\hline 10142 . & 400. & $0 .-250$. & 250. & 0. & 0 . \\
\hline 10016 . & 400 . & $0 .-100$. & 150. & 0 . & 0 . \\
\hline $989-25$ & 116. & $0 . \quad 0$. & 87. & 0 . & 0 . \\
\hline 105019. & 292 . & $0 .-100$. & 600. & 0. & 0 . \\
\hline 10503. & 700 . & $0 .-125$. & 325. & 0 . & 0 . \\
\hline 101435. & 553. & $0 .-200$. & 300. & 0. & 0 . \\
\hline 1011-11. & 0 & $0 . \quad 0$. & 0 & 0 . & 0 . \\
\hline 994-19. & -4 & -2 . & 2 . & 0 . & 0 . \\
\hline $983-20$. & 0 & 0 . & 0 . & 3. & 1 . \\
\hline $976-20$ & 0 . & 0 . & 0 . & 1. & 0 . \\
\hline 1011-11. & 0 . & 0 . & 0 . & 0 . & 0 . \\
\hline $1002-17$. & 0 . & 0 . & 0 . & 0. & 0 . \\
\hline 991-19. & 0 . & 0 . & 0 . & 0 . & 0 . \\
\hline $1002-17$. & 0 . & 0. & 0 . & 0 . & 0 . \\
\hline 988-19. & 0 . & 0 . & 0 . & 5. & 2 . \\
\hline $964-22$. & 0 . & 0 . & 0 . & 2. & 1 . \\
\hline $974-19$. & 0 . & 0 . & 0 . & 0 . & 0 . \\
\hline $970-21$. & 0 . & 0 . & 0 . & 1 . & 0 . \\
\hline $964-20$. & 0 . & 0 . & 0 . & 0 . & 0 . \\
\hline $965-20$. & 0 . & 0 . & 0 . & 0. & 0 . \\
\hline $931-25$. & 0 . & 0 . & 0 . & 2. & 1 . \\
\hline $944-24$ & 0 . & 0 . & 0 . & 1. & 0 . \\
\hline $1008-25$ & 0 . & 0 . & 0 . & 2. & 1. \\
\hline $997-21$ & 0 . & 0 . & 0 . & 2. & 1 . \\
\hline $950-23$. & 0 . & 0 . & 0 . & 2 . & 1 . \\
\hline $959-23$. & 0 . & 0 . & 0 . & 3. & 1. \\
\hline $957-23$. & 0 . & 0. & 0 . & 2 . & 1 . \\
\hline $939-24$. & 0 . & 0 . & 0 . & 3. & 1. \\
\hline $963-21$. & 0 . & 0 . & 0 . & 1. & 0 . \\
\hline $950-22$. & 0 . & 0 . & 0 . & 1. & 0 . \\
\hline $964-21$. & 0 . & 0 . & 0 . & 2. & 1. \\
\hline $978-20$ & 0 & $0 . \quad 0$. & 0 & 0 . & 0 . \\
\hline $1000-19$. & -36 & 0. -57 . & 57. & 0. & 0. \\
\hline $978-17$ & 0 . & $0 . \quad 0$. & 0 . & 30. & 23. \\
\hline $1000-18$. & -26 . & 0. -13 . & 13. & 0. & 0 . \\
\hline $1100-7$. & 50 & $0 .-100$ & 100. & 0 . & 0 . \\
\hline $1000-8$. & 8. & $0 . \quad-6$. & 6 & 0. & 0 . \\
\hline $975-20$ & 0 . & $0 . \quad 0$. & 0 . & 1 . & 0 . \\
\hline $980-20$. & 0 . & 0 . & 0 . & 1 . & 0 . \\
\hline 979-19. & 0 . & 0 . & 0 . & 4. & 1 . \\
\hline $1040-18$. & 0 . & 0. & 0 . & 1. & 0 . \\
\hline
\end{tabular}




\begin{tabular}{|c|c|c|c|c|c|}
\hline 37 & 9001 & 0.01 & 0.05 & 0.0000 .0000 .0000 .000 & \\
\hline 9001 & 9005 & 0.08 & 0.35 & 0.0000 .0000 .0000 .000 & 0 . \\
\hline 9001 & 9006 & 2.44 & 43.68 & 0.0000 .0000 .0000 .000 & 0 . \\
\hline 9001 & 9012 & 3.62 & 64.90 & 0.0000 .0000 .0000 .000 & 0 . \\
\hline 9005 & 9051 & 1.58 & 37.49 & 0.0000 .0000 .0000 .000 & 0 . \\
\hline 9005 & 9052 & 1.58 & 37.49 & 0.0000 .0000 .0000 .000 & 0 . \\
\hline 9005 & 9053 & 1.60 & 38.05 & 0.0000 .0000 .0000 .000 & 0 . \\
\hline 9005 & 9054 & 0.00 & 15.20 & 0.0000 .0000 .0000 .000 & 0 . \\
\hline 9005 & 9055 & 0.00 & 80.00 & 0.0000 .0000 .0000 .000 & 0 . \\
\hline 9006 & 9007 & 5.56 & 24.67 & 0.0000 .0000 .0000 .000 & 0 . \\
\hline 9006 & 9003 & 5.56 & 24.67 & 0.0000 .0000 .0000 .000 & 0 . \\
\hline 9012 & 9002 & 3.81 & 21.65 & 0.0000 .0000 .0000 .000 & \\
\hline 9002 & 9021 & 5.37 & 7.03 & 0.0000 .0000 .0000 .000 & 0 . \\
\hline 9021 & 9023 & 110.68 & 95.28 & 0.0000 .0000 .0000 .000 & 0 . \\
\hline 9021 & 9022 & 44.362 & 281.52 & 0.0000 .0000 .0000 .000 & 0 . \\
\hline 9002 & 9024 & 50.753 & 322.02 & 0.0000 .0000 .0000 .000 & 0 . \\
\hline 9023 & 9025 & 66.693 & 394.40 & 0.0000 .0000 .0000 .000 & 0 . \\
\hline 9023 & 9026 & 61.133 & 361.52 & 0.0000 .0000 .0000 .000 & 0 . \\
\hline 9007 & 9071 & 44.122 & 296.68 & 0.0000 .0000 .0000 .000 & 0 . \\
\hline 9007 & 9072 & 30.792 & 205.70 & 0.0000 .0000 .0000 .000 & 0 . \\
\hline 9007 & 9003 & 5.58 & 24.67 & 0.0000 .0000 .0000 .000 & 0 . \\
\hline 9003 & 9031 & 73.634 & 467.24 & 0.0000 .0000 .0000 .000 & 0 . \\
\hline 9003 & 9032 & 76.984 & 488.46 & 0.0000 .0000 .0000 .000 & 0 . \\
\hline 9003 & 9033 & 75.734 & 480.56 & 0.0000 .0000 .0000 .000 & 0 . \\
\hline 9003 & 9044 & 7.38 & 6.35 & 0.0000 .0000 .0000 .000 & 0 . \\
\hline 9044 & 9004 & 3.83 & 2.89 & 0.0000 .0000 .0000 .000 & 0 . \\
\hline 9004 & 9041 & 36.612 & 245.60 & 0.0000 .0000 .0000 .000 & 0 . \\
\hline 9004 & 9042 & 105.935 & 545.36 & 0.0000 .0000 .0000 .000 & 0 . \\
\hline 9004 & 9043 & 15.671 & 169.94 & 0.0000 .0000 .0000 .000 & 0 . \\
\hline 9003 & 9034 & 13.011 & 139.12 & 0.0000 .0000 .0000 .000 & 0 . \\
\hline 9003 & 9035 & 54.483 & 345.72 & 0.0000 .0000 .0000 .000 & 0 . \\
\hline 9003 & 9036 & 15.431 & 167.29 & 0.0000 .0000 .0000 .000 & 0 . \\
\hline 9003 & 9037 & 38.492 & 257.12 & 0.0000 .0000 .0000 .000 & 0 . \\
\hline 9003 & 9038 & 44.122 & 296.68 & 0.0000 .0000 .0000 .000 & 0 . \\
\hline 9012 & 9121 & 23.55 & 99.04 & 0.0000 .0000 .0000 .000 & 0 . \\
\hline 9053 & 9533 & 0.00 & 75.00 & 0.0000 .0000 .0000 .000 & 0 . \\
\hline 1 & 5 & 0.10 & 0.60 & 0.0000 .0000 .0000 .000 & \\
\hline 2 & 6 & 0.10 & 0.90 & 0.0000 .0000 .0000 .000 & 0 . \\
\hline 2 & 8 & 0.60 & 2.70 & 5.4000 .0000 .0000 .000 & 0 . \\
\hline 3 & 7 & 0.00 & 0.30 & 0.0000 .0000 .0000 .000 & 0. \\
\hline 3 & 19 & 0.80 & 6.901 & 13.9000 .0000 .0000 .000 & 0 . \\
\hline 3 & 150 & 0.10 & 0.70 & 0.0000 .0000 .0000 .000 & 0 . \\
\hline 4 & 16 & 0.20 & 1.901 & 112.700 .0000 .0000 .000 & 0 . \\
\hline 5 & 9 & 0.60 & 2.90 & 1.8000 .0000 .0000 .000 & 0 . \\
\hline 7 & 12 & 0.10 & 0.90 & 7.0000 .0000 .0000 .000 & 0 . \\
\hline 7 & 131 & 0.10 & 0.70 & 1.4000 .0000 .0000 .000 & 0 . \\
\hline 8 & 11 & 1.30 & 5.95 & 3.3000 .0000 .0000 .000 & 0 . \\
\hline 8 & 14 & 1.30 & 4.20 & 8.1000 .0000 .0000 .000 & \\
\hline 9 & 11 & 0.60 & 2.70 & 1.3000 .0000 .0000 .000 & 0 . \\
\hline 11 & 13 & 0.80 & 3.40 & 1.8000 .0000 .0000 .000 & 0 . \\
\hline 12 & 21 & 0.20 & 1.501 & 11.8000 .0000 .0000 .000 & 0 . \\
\hline 13 & 20 & 0.60 & 3.40 & 1.6000 .0000 .0000 .000 & 0 . \\
\hline 14 & 15 & 1.40 & 4.20 & 9.7000 .0000 .0000 .000 & 0 . \\
\hline 15 & 37 & 6.50 & 24.801 & 12.1000 .0000 .0000 .000 & 0 . \\
\hline 15 & 89 & 9.90 & 24.80 & 3.5000 .0000 .0000 .000 & 0 . \\
\hline 15 & 90 & 9.60 & 36.30 & 4.8000 .0000 .0000 .000 & 0 . \\
\hline 16 & 42 & 0.20 & 2.201 & 128.000 .0000 .0000 .000 & 0 . \\
\hline 19 & 21 & 0.20 & 1.80 & 3.6000 .0000 .0000 .000 & 0 . \\
\hline 19 & 87 & 1.30 & 8.001 & 15.1000 .0000 .0000 .000 & 0 . \\
\hline 20 & 22 & 1.60 & 3.30 & 1.5000 .0000 .0000 .000 & 0 . \\
\hline 20 & 27 & 6.90 & 18.60 & 9.8000 .0000 .0000 .000 & 0 . \\
\hline 21 & 24 & 0.40 & 3.402 & 28.0000 .0000 .0000 .000 & 0 . \\
\hline 22 & 23 & 5.20 & 11.10 & 5.0000 .0000 .0000 .000 & 0 . \\
\hline 23 & 25 & 1.90 & 3.90 & 1.8000 .0000 .0000 .000 & 0 . \\
\hline 24 & 319 & 0.70 & 6.801 & 13.4000 .0000 .0000 .000 & 0. \\
\hline 25 & 26 & 3.60 & 7.10 & 3.4000 .0000 .0000 .000 & 0 . \\
\hline 26 & 27 & 4.50 & 12.00 & 6.5000 .0000 .0000 .000 & 0 . \\
\hline 26 & 320 & 4.30 & 13.00 & 1.4000 .0000 .0000 .000 & 0 . \\
\hline 33 & 34 & 0.00 & 6.30 & 0.0000 .0000 .0000 .000 & 0 . \\
\hline 33 & 38 & 0.25 & 1.20 & 1.3000 .0000 .0000 .000 & 0 . \\
\hline 33 & 40 & 0.60 & 2.90 & 2.0000 .0000 .0000 .000 & \\
\hline 33 & 41 & 0.70 & 4.30 & 2.6000 .0000 .0000 .000 & \\
\hline 34 & 42 & 0.10 & 0.80 & 4.2000 .0000 .0000 .000 & \\
\hline 35 & 72 & 1.20 & 6.00 & 0.8000 .0000 .0000 .000 & \\
\hline
\end{tabular}




\begin{tabular}{|c|c|c|c|c|}
\hline 35 & 76 & 0.60 & 1.400 .2000 .0000 .0000 .000 & 0 . \\
\hline 35 & 77 & 1.00 & 2.900 .3000 .0000 .0000 .000 & 0 . \\
\hline 36 & 88 & 0.40 & 2.704 .3000 .0000 .0000 .000 & 0 . \\
\hline 37 & 38 & 0.80 & 4.700 .8000 .0000 .0000 .000 & 0 . \\
\hline 37 & 40 & 2.20 & 6.400 .7000 .0000 .0000 .000 & 0 . \\
\hline 37 & 41 & 1.00 & 3.602 .0000 .0000 .0000 .000 & 0 . \\
\hline 37 & 49 & 1.70 & 8.104 .8000 .0000 .0000 .000 & 0 . \\
\hline 37 & 89 & 10.20 & 25.403 .3000 .0000 .0000 .000 & 0 . \\
\hline 37 & 90 & 4.70 & 12.701 .6000 .0000 .0000 .000 & 0 . \\
\hline 38 & 41 & 0.80 & 3.702 .0000 .0000 .0000 .000 & 0 . \\
\hline 38 & 43 & 3.20 & 8.704 .0000 .0000 .0000 .000 & 0 . \\
\hline 39 & 42 & 0.06 & 0.6440 .4000 .0000 .0000 .000 & 0 . \\
\hline 40 & 48 & 2.60 & 15.402 .2000 .0000 .0000 .000 & 0 . \\
\hline 41 & 42 & 0.00 & 2.900 .0000 .0000 .0000 .000 & 0 . \\
\hline 41 & 49 & 6.50 & 19.102 .0000 .0000 .0000 .000 & 0 . \\
\hline 41 & 51 & 3.10 & 8.903 .6000 .0000 .0000 .000 & 0 . \\
\hline 42 & 46 & 0.20 & 1.4080 .6000 .0000 .0000 .000 & 0 . \\
\hline 43 & 44 & 2.60 & 7.203 .5000 .0000 .0000 .000 & 0 . \\
\hline 43 & 48 & 9.50 & 26.203 .2000 .0000 .0000 .000 & 0 . \\
\hline 43 & 53 & 1.30 & 3.901 .6000 .0000 .0000 .000 & 0 . \\
\hline 44 & 47 & 2.70 & 8.403 .9000 .0000 .0000 .000 & 0 . \\
\hline 44 & 54 & 2.80 & 8.403 .7000 .0000 .0000 .000 & 0 . \\
\hline 45 & 60 & 0.70 & 4.1031 .2000 .0000 .0000 .000 & 0 . \\
\hline 45 & 74 & 0.90 & 5.4041 .1000 .0000 .0000 .000 & 0 . \\
\hline 46 & 81 & 0.50 & 4.2069 .0000 .0000 .0000 .000 & 0 . \\
\hline 47 & 73 & 5.20 & 14.507 .3000 .0000 .0000 .000 & 0 . \\
\hline 47 & 113 & 4.30 & 11.801 .3000 .0000 .0000 .000 & 0 . \\
\hline 48 & 107 & 2.50 & 6.200 .7000 .0000 .0000 .000 & 0 . \\
\hline 49 & 51 & 3.10 & 9.404 .3000 .0000 .0000 .000 & 0 . \\
\hline 51 & 52 & 3.70 & 10.904 .9000 .0000 .0000 .000 & 0 . \\
\hline 52 & 55 & 2.70 & 8.003 .6000 .0000 .0000 .000 & 0 . \\
\hline 53 & 54 & 2.50 & 7.303 .5000 .0000 .0000 .000 & 0 . \\
\hline 54 & 55 & 3.50 & 10.304 .7000 .0000 .0000 .000 & 0 . \\
\hline 55 & 57 & 6.50 & 16.908 .2000 .0000 .0000 .000 & 0 . \\
\hline 57 & 58 & 4.60 & 8.003 .6000 .0000 .0000 .000 & 0 . \\
\hline 57 & 63 & 15.90 & 53.707 .1000 .0000 .0000 .000 & 0 . \\
\hline 58 & 59 & 0.90 & 2.600 .5000 .0000 .0000 .000 & 0 . \\
\hline 59 & 61 & 0.20 & 1.301 .5000 .0000 .0000 .000 & 0 . \\
\hline 60 & 62 & 0.90 & 6.5048 .5000 .0000 .0000 .000 & 0 . \\
\hline 62 & 64 & 1.60 & 10.5020 .3000 .0000 .0000 .000 & 0 . \\
\hline 62 & 144 & 0.10 & 0.701 .3000 .0000 .0000 .000 & 0 . \\
\hline 63 & 526 & 2.65 & 17.202 .6000 .0000 .0000 .000 & 0 . \\
\hline 69 & 211 & 5.10 & 23.202 .8000 .0000 .0000 .000 & 0 . \\
\hline 69 & 79 & 5.10 & 15.702 .3000 .0000 .0000 .000 & 0 . \\
\hline 70 & 71 & 3.20 & 10.006 .2000 .0000 .0000 .000 & 0 . \\
\hline 70 & 528 & 2.00 & 12.342 .8000 .0000 .0000 .000 & 0 . \\
\hline 71 & 72 & 3.60 & 13.106 .8000 .0000 .0000 .000 & 0 . \\
\hline 71 & 73 & 3.40 & 9.904 .7000 .0000 .0000 .000 & 0 . \\
\hline 72 & 77 & 1.80 & 8.701 .1000 .0000 .0000 .000 & 0 . \\
\hline 72 & 531 & 2.56 & 19.300 .0000 .0000 .0000 .000 & 0 . \\
\hline 73 & 76 & 2.10 & 5.703 .0000 .0000 .0000 .000 & 0 . \\
\hline 73 & 79 & 1.80 & 5.201 .8000 .0000 .0000 .000 & 0 . \\
\hline 74 & 88 & 0.40 & 2.705 .0000 .0000 .0000 .000 & 0 . \\
\hline 74 & 562 & 2.86 & 20.1337 .9000 .0000 .0000 .000 & 0 . \\
\hline 76 & 77 & 1.60 & 4.300 .4000 .0000 .0000 .000 & 0 . \\
\hline 77 & 78 & 0.10 & 0.600 .7000 .0000 .0000 .000 & 0 . \\
\hline 77 & 80 & 1.40 & 7.003 .8000 .0000 .0000 .000 & 0 . \\
\hline 77 & 552 & 8.91 & 26.762 .9000 .0000 .0000 .000 & 0 . \\
\hline 77 & 609 & 7.82 & 21.272 .2000 .0000 .0000 .000 & 0 . \\
\hline 78 & 79 & 0.60 & 2.201 .1000 .0000 .0000 .000 & 0 . \\
\hline 78 & 84 & 0.00 & 3.600 .0000 .0000 .0000 .000 & 0 . \\
\hline 79 & 211 & 9.90 & 37.505 .1000 .0000 .0000 .000 & 0 . \\
\hline 80 & 211 & 2.20 & 10.705 .8000 .0000 .0000 .000 & 0 . \\
\hline 81 & 194 & 0.35 & 3.3053 .0000 .0000 .0000 .000 & 0 . \\
\hline 81 & 195 & 0.35 & 3.3053 .0000 .0000 .0000 .000 & 0 . \\
\hline 85 & 86 & 0.80 & 6.4012 .8000 .0000 .0000 .000 & 0 . \\
\hline 86 & 87 & 1.20 & 9.3018 .3000 .0000 .0000 .000 & 0 . \\
\hline 86 & 323 & 0.60 & 4.809 .2000 .0000 .0000 .000 & 0 . \\
\hline 89 & 91 & 4.70 & 11.901 .4000 .0000 .0000 .000 & 0 . \\
\hline 90 & 92 & 3.20 & 17.402 .4000 .0000 .0000 .000 & 0 . \\
\hline 91 & 94 & 10.00 & 25.303 .1000 .0000 .0000 .000 & 0 . \\
\hline 91 & 97 & 2.20 & 7.703 .9000 .0000 .0000 .000 & 0 . \\
\hline 92 & 103 & 1.90 & 14.401 .7000 .0000 .0000 .000 & 0 . \\
\hline 92 & 105 & 1.70 & 9.201 .2000 .0000 .0000 .000 & \\
\hline
\end{tabular}




\begin{tabular}{|c|c|c|c|c|c|}
\hline 94 & 97 & 27.80 & 42.70 & 4.3000 .0000 .0000 .000 & 0 . \\
\hline 97 & 100 & 2.20 & 5.30 & 0.7000 .0000 .0000 .000 & 0. \\
\hline 97 & 102 & 3.80 & 9.20 & 1.2000 .0000 .0000 .000 & 0 . \\
\hline 97 & 103 & 4.80 & 12.20 & 1.5000 .0000 .0000 .000 & 0. \\
\hline 98 & 100 & 2.40 & 6.40 & 0.7000 .0000 .0000 .000 & 0. \\
\hline 98 & 102 & 3.40 & 12.10 & 1.5000 .0000 .0000 .000 & 0 . \\
\hline 99 & 107 & 5.30 & 13.50 & 1.7000 .0000 .0000 .000 & 0 . \\
\hline 99 & 108 & 0.20 & 0.40 & 0.2000 .0000 .0000 .000 & 0. \\
\hline 99 & 109 & 4.50 & 35.40 & 4.4000 .0000 .0000 .000 & \\
\hline 99 & 110 & 5.00 & 17.40 & 2.2000 .0000 .0000 .000 & b. \\
\hline 100 & 102 & 1.60 & 3.80 & 0.4000 .0000 .0000 .000 & 0. \\
\hline 102 & 104 & 4.30 & 6.40 & 2.7000 .0000 .0000 .000 & \\
\hline 103 & 105 & 1.90 & 6.20 & 0.8000 .0000 .0000 .000 & 0. \\
\hline 104 & 108 & 7.60 & 13.00 & 4.4000 .0000 .0000 .000 & 0 . \\
\hline 104 & 322 & 4.40 & 12.40 & 1.5000 .0000 .0000 .000 & 0. \\
\hline 105 & 107 & 1.20 & 8.80 & 1.1000 .0000 .0000 .000 & 0. \\
\hline 105 & 110 & 15.70 & 40.00 & 4.7000 .0000 .0000 .000 & 0. \\
\hline 108 & 324 & 7.40 & 20.80 & 2.6000 .0000 .0000 .000 & 0. \\
\hline 109 & 110 & 7.00 & 18.40 & 2.1000 .0000 .0000 .000 & 0. \\
\hline 109 & 113 & 10.00 & 27.40 & 3.1000 .0000 .0000 .000 & 0. \\
\hline 109 & 114 & 10.90 & 39.30 & 3.6000 .0000 .0000 .000 & 0. \\
\hline 110 & 112 & 14.20 & 40.40 & 5.0000 .0000 .0000 .000 & 0. \\
\hline 112 & 114 & 1.70 & 4.20 & 0.6000 .0000 .0000 .000 & 0. \\
\hline 115 & 122 & 0.36 & 1.99 & 0.4000 .0000 .0000 .000 & b. \\
\hline 116 & 120 & 0.20 & 10.49 & 0.1000 .0000 .0000 .000 & 0. \\
\hline 117 & 118 & 0.01 & 0.18 & 1.7000 .0000 .0000 .000 & b. \\
\hline 118 & 119 & 0.00 & 2.71 & 0.0000 .0000 .0000 .000 & 0. \\
\hline 118 & 1201 & 0.00 & 61.63 & 0.0000 .0000 .0000 .000 & 0 . \\
\hline 1201 & 120 & $0.00-$ & -36.97 & 0.0000 .0000 .0000 .000 & 0. \\
\hline 118 & 121 & 0.22 & 29.15 & 0.0000 .0000 .0000 .000 & 0 . \\
\hline 119 & 120 & 0.00 & 3.39 & 0.0000 .0000 .0000 .000 & 0. \\
\hline 119 & 121 & 0.00 & 5.82 & 0.0000 .0000 .0000 .000 & 0 . \\
\hline 122 & 123 & 8.08 & 23.44 & 2.9000 .0000 .0000 .000 & 0. \\
\hline 122 & 125 & 9.65 & 36.69 & 5.4000 .0000 .0000 .000 & 0. \\
\hline 123 & 124 & 3.60 & 10.761 & 11.7000 .0000 .0000 .000 & 0. \\
\hline 123 & 125 & 4.76 & 14.141 & 14.9000 .0000 .0000 .000 & 0. \\
\hline 125 & 126 & 0.06 & 1.97 & 0.0000 .0000 .0000 .000 & 0. \\
\hline 126 & 127 & 0.59 & 4.052 & 25.0000 .0000 .0000 .000 & 0. \\
\hline 126 & 129 & 1.15 & 11.061 & 18.5000 .0000 .0000 .000 & 0. \\
\hline 126 & 132 & 1.98 & 16.883 & 32.1000 .0000 .0000 .000 & 0 . \\
\hline 126 & 157 & 0.50 & 5.003 & 33.0000 .0000 .0000 .000 & 0 . \\
\hline 126 & 158 & 0.77 & 5.383 & 33.5000 .0000 .0000 .000 & 0. \\
\hline 126 & 169 & 1.65 & 11.571 & 17.1000 .0000 .0000 .000 & 0 . \\
\hline 127 & 128 & 0.59 & 5.77 & 9.5000 .0000 .0000 .000 & 0. \\
\hline 127 & 134 & 0.49 & 3.362 & 20.8000 .0000 .0000 .000 & 0 . \\
\hline 127 & 168 & 0.59 & 5.77 & 9.5000 .0000 .0000 .000 & 0. \\
\hline 128 & 130 & 0.78 & 7.731 & 12.6000 .0000 .0000 .000 & 0 . \\
\hline 128 & 133 & 0.26 & 1.93 & 3.0000 .0000 .0000 .000 & 0. \\
\hline 129 & 130 & 0.76 & 7.521 & 12.2000 .0000 .0000 .000 & 0 . \\
\hline 129 & 133 & 0.21 & 1.86 & 3.0000 .0000 .0000 .000 & 0. \\
\hline 130 & 132 & 0.16 & 1.64 & 2.6000 .0000 .0000 .000 & 0 . \\
\hline 130 & 151 & 0.17 & 1.65 & 2.6000 .0000 .0000 .000 & 0 . \\
\hline 130 & 167 & 0.79 & 7.931 & 12.7000 .0000 .0000 .000 & 0. \\
\hline 130 & 168 & 0.78 & 7.841 & 12.5000 .0000 .0000 .000 & 0 . \\
\hline 133 & 137 & 0.17 & 1.172 & 28.9000 .0000 .0000 .000 & 0. \\
\hline 133 & 168 & 0.26 & 1.93 & 3.0000 .0000 .0000 .000 & 0. \\
\hline 133 & 169 & 0.21 & 1.86 & 3.0000 .0000 .0000 .000 & 0. \\
\hline 133 & 171 & 0.02 & 1.01 & 0.0000 .0000 .0000 .000 & 0. \\
\hline 134 & 135 & 0.43 & 2.931 & 18.0000 .0000 .0000 .000 & 0. \\
\hline 134 & 184 & 0.39 & 3.812 & 25.8000 .0000 .0000 .000 & 0. \\
\hline 135 & 136 & 0.91 & 6.233 & 38.5000 .0000 .0000 .000 & 0. \\
\hline 136 & 137 & 1.25 & 8.905 & 54.0000 .0000 .0000 .000 & 0 . \\
\hline 136 & 152 & 0.56 & 3.909 & 95.3000 .0000 .0000 .000 & 0. \\
\hline 137 & 140 & 0.15 & 1.142 & 28.4000 .0000 .0000 .000 & \\
\hline 137 & 181 & 0.05 & 0.34 & 2.1000 .0000 .0000 .000 & 0 . \\
\hline 137 & 186 & 0.07 & 1.511 & 12.6000 .0000 .0000 .000 & 0. \\
\hline 137 & 188 & 0.05 & 0.34 & 2.1000 .0000 .0000 .000 & \\
\hline 139 & 172 & 5.62 & 22.48 & 8.1000 .0000 .0000 .000 & 0. \\
\hline 140 & 141 & 1.20 & 8.361 & 12.3000 .0000 .0000 .000 & 0. \\
\hline 140 & 142 & 1.52 & 11.326 & 68.4000 .0000 .0000 .000 & 0 . \\
\hline 140 & 145 & 4.68 & 33.695 & 51.9000 .0000 .0000 .000 & b. \\
\hline 140 & 146 & 4.30 & 30.314 & 46.3000 .0000 .0000 .000 & \\
\hline 140 & 147 & 4.89 & 34.925 & 53.8000 .0000 .0000 .000 & \\
\hline 140 & 182 & 0.13 & 0.891 & 11.9000 .0000 .0000 .000 & \\
\hline
\end{tabular}




\begin{tabular}{|c|c|c|c|c|}
\hline 141 & 146 & 2.91 & 22.6734 .2000 .0000 .0000 .000 & 0. \\
\hline 142 & 143 & 0.60 & 5.7076 .7000 .0000 .0000 .000 & 0 . \\
\hline 143 & 145 & 0.75 & 7.7311 .9000 .0000 .0000 .000 & 0 . \\
\hline 143 & 149 & 1.27 & 9.0913 .5000 .0000 .0000 .000 & 0 . \\
\hline 145 & 146 & 0.85 & 5.888 .7000 .0000 .0000 .000 & 0. \\
\hline 145 & 149 & 2.18 & 15.1122 .3000 .0000 .0000 .000 & 0 . \\
\hline 146 & 147 & 0.73 & 5.047 .4000 .0000 .0000 .000 & 0 . \\
\hline 148 & 178 & 5.23 & 15.267 .4000 .0000 .0000 .000 & 0 . \\
\hline 148 & 179 & 13.71 & 39.197 .6000 .0000 .0000 .000 & 0 . \\
\hline 152 & 153 & 1.37 & 9.5714 .1000 .0000 .0000 .000 & 0 . \\
\hline 153 & 161 & 0.55 & 2.8819 .0000 .0000 .0000 .000 & 0 . \\
\hline 154 & 156 & 17.46 & 31.614 .0000 .0000 .0000 .000 & 0 . \\
\hline 154 & 183 & 8.04 & 30.544 .5000 .0000 .0000 .000 & 0 . \\
\hline 155 & 161 & 1.10 & 5.6838 .8000 .0000 .0000 .000 & 0 . \\
\hline 157 & 159 & 0.08 & 0.986 .9000 .0000 .0000 .000 & 0 . \\
\hline 158 & 159 & 0.29 & 2.8519 .0000 .0000 .0000 .000 & 0. \\
\hline 158 & 160 & 0.66 & 4.4827 .7000 .0000 .0000 .000 & 0. \\
\hline 162 & 164 & 0.24 & 3.2623 .6000 .0000 .0000 .000 & 0 . \\
\hline 162 & 165 & 0.18 & 2.45166 .200 .0000 .0000 .000 & 0 . \\
\hline 163 & 164 & 0.44 & 5.14359 .700 .0000 .0000 .000 & 0 . \\
\hline 165 & 166 & 0.02 & 1.230 .0000 .0000 .0000 .000 & 0 . \\
\hline 167 & 169 & 0.18 & 1.782 .9000 .0000 .0000 .000 & 0. \\
\hline 172 & 173 & 6.69 & 48.436 .3000 .0000 .0000 .000 & 0 . \\
\hline 172 & 174 & 5.58 & 22.103 .1000 .0000 .0000 .000 & 0 . \\
\hline 173 & 174 & 8.07 & 33.314 .9000 .0000 .0000 .000 & 0 . \\
\hline 173 & 175 & 7.39 & 30.714 .3000 .0000 .0000 .000 & 0 . \\
\hline 173 & 176 & 17.99 & 50.176 .9000 .0000 .0000 .000 & 0 . \\
\hline 175 & 176 & 9.04 & 36.264 .8000 .0000 .0000 .000 & 0 . \\
\hline 175 & 179 & 7.70 & 30.925 .4000 .0000 .0000 .000 & 0 . \\
\hline 176 & 177 & 2.51 & 8.294 .7000 .0000 .0000 .000 & 0 . \\
\hline 177 & 178 & 2.22 & 8.475 .0000 .0000 .0000 .000 & 0 . \\
\hline 178 & 179 & 4.98 & 18.552 .9000 .0000 .0000 .000 & 0 . \\
\hline 178 & 180 & 0.61 & 2.908 .4000 .0000 .0000 .000 & 0 . \\
\hline 181 & 138 & 0.04 & 2.020 .0000 .0000 .0000 .000 & 0 . \\
\hline 181 & 187 & 0.04 & 0.8311 .5000 .0000 .0000 .000 & 0 . \\
\hline 184 & 185 & 0.25 & 2.4516 .4000 .0000 .0000 .000 & 0 . \\
\hline 186 & 188 & 0.07 & 0.8611 .5000 .0000 .0000 .000 & 0 . \\
\hline 187 & 188 & 0.07 & 0.8611 .5000 .0000 .0000 .000 & 0 . \\
\hline 188 & 138 & 0.04 & 2.020 .0000 .0000 .0000 .000 & 0 . \\
\hline 189 & 208 & 3.30 & 9.500 .0000 .0000 .0000 .000 & 0 . \\
\hline 189 & 209 & 4.60 & 6.900 .0000 .0000 .0000 .000 & 0 . \\
\hline 190 & 231 & 0.04 & 0.22620 .000 .0000 .0000 .000 & 0. \\
\hline 190 & 240 & 0.00 & 2.750 .0000 .0000 .0000 .000 & 0 . \\
\hline 191 & 192 & 0.30 & 4.800 .0000 .0000 .0000 .000 & 0 . \\
\hline 192 & 225 & 0.20 & 0.900 .0000 .0000 .0000 .000 & 0 . \\
\hline 193 & 205 & 4.50 & 6.300 .0000 .0000 .0000 .000 & 0 . \\
\hline 193 & 208 & 4.80 & 12.700 .0000 .0000 .0000 .000 & 0 . \\
\hline 194 & 219 & 0.31 & 2.8650 .0000 .0000 .0000 .000 & 0 . \\
\hline 194 & 664 & 0.24 & 3.5536 .0000 .0000 .0000 .000 & 0 . \\
\hline 195 & 219 & 0.31 & 2.8650 .0000 .0000 .0000 .000 & 0 . \\
\hline 196 & 197 & 1.40 & 4.000 .4000 .0000 .0000 .000 & 0 . \\
\hline 196 & 210 & 3.00 & 8.101 .0000 .0000 .0000 .000 & 0 . \\
\hline 197 & 198 & 1.00 & 6.000 .9000 .0000 .0000 .000 & 0. \\
\hline 197 & 211 & 1.50 & 4.000 .6000 .0000 .0000 .000 & 0 . \\
\hline 198 & 202 & 33.20 & 0.0000 .0000 .0000 .000 & 0 . \\
\hline 198 & 203 & 0.90 & 2.5000 .0000 .0000 .000 & 0 . \\
\hline 198 & 210 & 2.00 & 7.300 .8000 .0000 .0000 .000 & 0 . \\
\hline 198 & 211 & 3.40 & 3.2000 .0000 .0000 .000 & 0 . \\
\hline 199 & 200 & 7.60 & 13.500 .9000 .0000 .0000 .000 & 0 . \\
\hline 199 & 210 & 4.00 & 10.200 .5000 .0000 .0000 .000 & 0 . \\
\hline 200 & 210 & 8.10 & 12.801 .4000 .0000 .0000 .000 & 0 . \\
\hline 201 & 204 & 12.40 & 0.0000 .0000 .0000 .000 & 0 . \\
\hline 203 & 211 & 1.00 & 5.900 .8000 .0000 .0000 .000 & 0 . \\
\hline 204 & 205 & 4.60 & 6.800 .0000 .0000 .0000 .000 & 0 . \\
\hline 205 & 206 & 30.20 & 0.0000 .0000 .0000 .000 & 0 . \\
\hline 206 & 207 & 7.30 & 0.0000 .0000 .0000 .000 & 0 . \\
\hline 206 & 208 & 24.00 & 42.100 .0000 .0000 .0000 .000 & 0 . \\
\hline 212 & 215 & 1.39 & 8.6000 .0000 .0000 .000 & 0 . \\
\hline 213 & 214 & 0.25 & 0.0000 .0000 .0000 .000 & 0 . \\
\hline 214 & 215 & 0.17 & 1.852 .0000 .0000 .0000 .000 & 0 . \\
\hline 214 & 242 & 0.15 & 1.080 .2000 .0000 .0000 .000 & 0 . \\
\hline 215 & 216 & 0.45 & 2.492 .6000 .0000 .0000 .000 & 0. \\
\hline 216 & 217 & 0.40 & 4.971 .8000 .0000 .0000 .000 & 0 . \\
\hline 217 & 218 & 0.00 & 4.560 .0000 .0000 .0000 .000 & 0 . \\
\hline
\end{tabular}




\begin{tabular}{|c|c|c|c|c|}
\hline 217 & 219 & 0.05 & 1.772 .0000 .0000 .0000 .000 & 0. \\
\hline 217 & 220 & 0.27 & 3.9583 .2000 .0000 .0000 .000 & 0 . \\
\hline 219 & 237 & 0.03 & 0.18520 .000 .0000 .0000 .000 & 0 . \\
\hline 220 & 218 & 0.37 & 4.8443 .0000 .0000 .0000 .000 & 0 . \\
\hline 220 & 221 & 0.10 & 2.9550 .3000 .0000 .0000 .000 & 0. \\
\hline 220 & 238 & 0.16 & 0.4640 .2000 .0000 .0000 .000 & 0. \\
\hline 221 & 223 & 0.03 & 0.13100 .000 .0000 .0000 .000 & 0 . \\
\hline 222 & 237 & 0.14 & 5.1433 .0000 .0000 .0000 .000 & 0 . \\
\hline 224 & 225 & 1.00 & 6.4048 .0000 .0000 .0000 .000 & 0. \\
\hline 224 & 226 & 0.19 & 0.8186 .0000 .0000 .0000 .000 & 0 . \\
\hline 225 & 191 & 0.10 & 6.100 .0000 .0000 .0000 .000 & 0 . \\
\hline 226 & 231 & 0.05 & 2.120 .0000 .0000 .0000 .000 & 0 . \\
\hline 227 & 231 & 0.09 & 4.7218 .6000 .0000 .0000 .000 & 0 . \\
\hline 228 & 229 & 0.19 & 0.87128 .000 .0000 .0000 .000 & 0 . \\
\hline 228 & 231 & 0.26 & 9.170 .0000 .0000 .0000 .000 & 0 . \\
\hline 228 & 234 & 0.13 & 2.8881 .0000 .0000 .0000 .000 & 0 . \\
\hline 229 & 190 & 0.00 & 6.260 .0000 .0000 .0000 .000 & 0 . \\
\hline 231 & 232 & 0.02 & 0.69136 .400 .0000 .0000 .000 & 0 . \\
\hline 231 & 237 & 0.01 & 0.06357 .00 .0000 .0000 .000 & 0 . \\
\hline 232 & 233 & 0.17 & 4.850 .0000 .0000 .0000 .000 & 0. \\
\hline 234 & 235 & 0.02 & 2.5914 .4000 .0000 .0000 .000 & 0 . \\
\hline 234 & 237 & 0.06 & 2.720 .0000 .0000 .0000 .000 & 0 . \\
\hline 235 & 238 & 0.02 & 0.0680 .0000 .0000 .0000 .000 & 0 . \\
\hline 241 & 237 & 0.05 & 1.540 .0000 .0000 .0000 .000 & 0 . \\
\hline 240 & 281 & 0.03 & 0.430 .9000 .0000 .0000 .000 & 0 . \\
\hline 242 & 245 & 0.82 & 8.510 .0000 .0000 .0000 .000 & 0 . \\
\hline 242 & 247 & 1.12 & 7.230 .0000 .0000 .0000 .000 & 0 . \\
\hline 243 & 244 & 1.27 & 3.550 .0000 .0000 .0000 .000 & 0 . \\
\hline 243 & 245 & 3.26 & 18.040 .0000 .0000 .0000 .000 & 0 . \\
\hline 244 & 246 & 1.95 & 0.0000 .0000 .0000 .000 & 0 . \\
\hline 245 & 246 & 1.57 & 7.320 .0000 .0000 .0000 .000 & 0 . \\
\hline 245 & 247 & 3.60 & 21.190 .0000 .0000 .0000 .000 & 0 . \\
\hline 246 & 247 & 2.68 & 12.850 .0000 .0000 .0000 .000 & 0 . \\
\hline 247 & 248 & 4.28 & 0.0000 .0000 .0000 .000 & 0 . \\
\hline 248 & 249 & 3.51 & 10.040 .0000 .0000 .0000 .000 & 0 . \\
\hline 249 & 250 & 6.16 & 18.570 .0000 .0000 .0000 .000 & 0 . \\
\hline 3 & 1 & 0.00 & 0.0000 .0000 .0000 .000 & 0 . \\
\hline 3 & 2 & 0.00 & 0.0000 .0000 .0000 .000 & 0 . \\
\hline 3 & 4 & 0.00 & 0.500 .0000 .0000 .0000 .000 & 0 . \\
\hline 7 & 5 & 0.00 & 0.0000 .0000 .0000 .000 & 0 . \\
\hline 7 & 6 & 0.00 & 0.0000 .0000 .0000 .000 & 0 . \\
\hline 10 & 11 & 0.00 & 0.0000 .0000 .0000 .000 & 0 . \\
\hline 12 & 10 & 0.00 & 5.300 .0000 .0000 .0000 .000 & 0 . \\
\hline 15 & 17 & 1.94 & 0.0000 .0000 .0000 .000 & 0 . \\
\hline 16 & 15 & 0.10 & 0.0000 .0000 .0000 .000 & 0 . \\
\hline 21 & 20 & 0.00 & 1.400 .0000 .0000 .0000 .000 & 0 . \\
\hline 24 & 23 & 0.00 & 6.400 .0000 .0000 .0000 .000 & 0 . \\
\hline 36 & 35 & 0.00 & 4.700 .0000 .0000 .0000 .000 & 0. \\
\hline 45 & 44 & 0.00 & 0.0000 .0000 .0000 .000 & 0 . \\
\hline 45 & 46 & 0.00 & 2.100 .0000 .0000 .0000 .000 & 0 . \\
\hline 62 & 61 & 0.00 & 0.0000 .0000 .0000 .000 & 0 . \\
\hline 63 & 64 & 0.00 & 0.0000 .0000 .0000 .000 & 0 . \\
\hline 73 & 74 & 0.00 & 2.440 .0000 .0000 .0000 .000 & 0. \\
\hline 81 & 88 & 0.00 & 2.000 .0000 .0000 .0000 .000 & 0 . \\
\hline 85 & 99 & 0.00 & 0.0000 .0000 .0000 .000 & 0 . \\
\hline 86 & 102 & 0.00 & 4.800 .0000 .0000 .0000 .000 & 0 . \\
\hline 87 & 94 & 0.00 & 4.600 .0000 .0000 .0000 .000 & 0 . \\
\hline 114 & 207 & 0.00 & 0.0000 .0000 .0000 .000 & 0 . \\
\hline 116 & 124 & 0.52 & 0.0000 .0000 .0000 .000 & 0 . \\
\hline 121 & 115 & 0.00 & 2.800 .0000 .0000 .0000 .000 & 0 . \\
\hline 122 & 157 & 0.05 & 1.950 .0000 .0000 .0000 .000 & 0 . \\
\hline 130 & 131 & 0.00 & 0.0000 .0000 .0000 .000 & 0 . \\
\hline 130 & 150 & 0.00 & 1.400 .0000 .0000 .0000 .000 & 0 . \\
\hline 132 & 170 & 0.10 & 4.020 .0000 .0000 .0000 .000 & 0 . \\
\hline 141 & 174 & 0.24 & 6.030 .0000 .0000 .0000 .000 & 0 . \\
\hline 142 & 175 & 0.24 & $4.98-8.7000 .0000 .0000 .000$ & 0 . \\
\hline 143 & 144 & 0.00 & 8.330 .0000 .0000 .0000 .000 & 0 . \\
\hline 143 & 148 & 0.13 & 3.710 .0000 .0000 .0000 .000 & 0 . \\
\hline 145 & 180 & 0.05 & 1.820 .0000 .0000 .0000 .000 & 0 . \\
\hline 151 & 170 & 0.10 & 3.920 .0000 .0000 .0000 .000 & 0 . \\
\hline 153 & 183 & 0.27 & 6.390 .0000 .0000 .0000 .000 & 0 . \\
\hline 155 & 156 & 0.08 & 2.560 .0000 .0000 .0000 .000 & 0 . \\
\hline 159 & 117 & 0.00 & 0.0000 .0000 .0000 .000 & 0 . \\
\hline 160 & 124 & 0.12 & 3.960 .0000 .0000 .0000 .000 & 0 . \\
\hline
\end{tabular}


$0.13 \quad 3.84-5.7000 .0000 .0000 .000$

$0.092 .31-3.3000 .0000 .0000 .000 \quad 0$.

$0.031 .310 .0000 .0000 .0000 .000 \quad 0$.

$0.0025 .20 \quad 0.0000 .0000 .0000 .000$

$0.0023 .700 .0000 .0000 .0000 .000 \quad 0$.

$0.08 \quad 3.660 .0000 .0000 .0000 .000$

$0.0022 .00 \quad 0.0000 .0000 .0000 .000 \quad 0$.

$0.00 \quad 9.80 \quad 0.0000 .0000 .0000 .000 \quad 0$.

$0.0012 .80 \quad 0.0000 .0000 .0000 .000$

$2.0020 .40-1.2000 .0000 .0000 .000 \quad 0$.

2.6021 .100 .0000 .0000 .0000 .000

$0.30 \quad 1.220 .0000 .0000 .0000 .000 \quad 0$.

$0.103 .54-1.0000 .0000 .0000 .000 \quad 0$.

$0.121 .95-36.400 .0000 .0000 .000 \quad 0$.

$0.103 .320 .0000 .0000 .0000 .000 \quad 0$.

$0.051 .600 .0000 .0000 .0000 .000 \quad 0$.

$0.051 .600 .0000 .0000 .0000 .000 \quad 0$.

$0.012 .00 \quad 0.0000 .0000 .0000 .000 \quad 0$.

$0.10 \quad 2.30 \quad 0.0000 .0000 .0000 .000 \quad 0$.

$0.00 \quad 2.300 .0000 .0000 .0000 .000 \quad 0$.

$0.10 \quad 1.460 .0000 .0000 .0000 .000 \quad 0$.

$0.001 .050 .0000 .0000 .0000 .000 \quad 0$.

$0.00 \quad 2.38 \quad 0.0000 .0000 .0000 .000 \quad 0$.

$0.00 \quad 3.210 .0000 .0000 .0000 .000 \quad 0$.

$0.00 \quad 1.540 .0000 .0000 .0000 .000 \quad 0$.

$0.00 \quad 2.890 .0000 .0000 .0000 .000 \quad 0$.

$0.001 .950 .0000 .0000 .0000 .000 \quad 0$.

$0.00 \quad 1.930 .0000 .0000 .0000 .000 \quad 0$.

$0.00 \quad 1.920 .0000 .0000 .0000 .000 \quad 0$.

$0.00 \quad 2.30 \quad 0.0000 .0000 .0000 .000 \quad 0$.

$0.00 \quad 1.240 .0000 .0000 .0000 .000 \quad 0$.

$0.00 \quad 1.67 \quad 0.0000 .0000 .0000 .000 \quad 0$.

$0.00 \quad 3.120 .0000 .0000 .0000 .000 \quad 0$.

$0.00 \quad 1.650 .0000 .0000 .0000 .000 \quad 0$.

$0.003 .160 .0000 .0000 .0000 .000 \quad 0$.

$0.00 \quad 5.350 .0000 .0000 .0000 .000 \quad 0$.

$0.0018 .180 .0000 .0000 .0000 .000 \quad 0$.

$\begin{array}{rrrr}0.00 & 19.61 & 0.0000 .0000 .0000 .000 & 0 . \\ 0.00 & 6.90 & 0.0000 .0000 .0000 .000 & 0 .\end{array}$

$0.90 \quad 1.1$

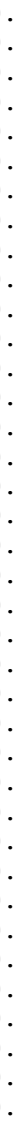




\section{RESULTADOS OBTIDOS}

Neste apêndice estão apresentados os resultados obtidos pelo programa de FPO para o ponto inicial, e os resultados obtidos pelo programa SFPO após as perturbações nos sistemas.

\section{SISTEMA IEEE 118 BARRAS}

TABELA B.1 - Solução ótima do sistema IEEE 118 barras obtida pelo FPO para $(\varepsilon=0,0)$ e utilizada na SFPO

\begin{tabular}{|c|c|c|}
\hline Barra k & $\mathrm{V}_{\mathrm{k}}$ (p.u.) & $\theta_{\mathrm{k}}$ (graus) \\
\hline \hline 69 & 1,0167 & 30,0000 \\
\hline 1 & 0,9589 & 10,7027 \\
\hline 2 & 0,9715 & 11,2973 \\
\hline 3 & 0,9688 & 11,6346 \\
\hline 4 & 0,9998 & 15,3660 \\
\hline 5 & 0,9983 & 15,8873 \\
\hline 6 & 0,9910 & 13,0810 \\
\hline 7 & 0,9891 & 12,6477 \\
\hline 8 & 1,0043 & 21,0802 \\
\hline 9 & 1,0380 & 28,4219 \\
\hline 10 & 1,0517 & 35,9975 \\
\hline 11 & 0,9834 & 12,8242 \\
\hline 12 & 0,9878 & 12,3099 \\
\hline 13 & 0,9672 & 11,4141 \\
\hline 14 & 0,9822 & 11,5538 \\
\hline 15 & 0,9710 & 11,1546 \\
\hline 16 & 0,9799 & 12,0028 \\
\hline
\end{tabular}


continuação

\begin{tabular}{|c|c|c|}
\hline 17 & 0,9872 & 13,8236 \\
\hline 18 & 0,9705 & 11,5038 \\
\hline 19 & 0,9656 & 10,9519 \\
\hline 20 & 0,9596 & 11,8260 \\
\hline 21 & 0,9597 & 13,4079 \\
\hline 22 & 0,9702 & 15,9563 \\
\hline 23 & 0,9992 & 20,8736 \\
\hline 24 & 0,9953 & 20,6872 \\
\hline 25 & 1,0413 & 27,9472 \\
\hline 26 & 1,0188 & 29,7900 \\
\hline 27 & 0,9688 & 15,2234 \\
\hline 28 & 0,9612 & 13,5271 \\
\hline 29 & 0,9615 & 12,5654 \\
\hline 30 & 1,0050 & 18,9857 \\
\hline 31 & 0,9648 & 12,6964 \\
\hline 32 & 0,9663 & 14,6516 \\
\hline 33 & 0,9704 & 10,5608 \\
\hline 34 & 0,9862 & 11,1758 \\
\hline 35 & 0,9828 & 10,7219 \\
\hline 36 & 0,9833 & 10,7078 \\
\hline 37 & 0,9884 & 11,7047 \\
\hline 38 & 1,0013 & 17,0502 \\
\hline 39 & 0,9716 & 8,1801 \\
\hline 40 & 0,9739 & 7,0285 \\
\hline 41 & 0,9696 & 6,5823 \\
\hline 42 & 0,9844 & 8,1667 \\
\hline 43 & 0,9786 & 11,0444 \\
\hline 44 & 0,9847 & 13,4055 \\
\hline 45 & 0,9862 & 15,1920 \\
\hline 46 & 1,0065 & 17,9404 \\
\hline 47 & 1,0127 & 20,2628 \\
\hline 48 & 1,0180 & 19,4355 \\
\hline 49 & 1,0213 & 20,4594 \\
\hline 50 & 0,9983 & 18,3448 \\
\hline 51 & 0,9658 & 15,6256 \\
\hline 52 & 0,9564 & 14,6410 \\
\hline 53 & 0,9476 & 13,5961 \\
\hline 54 & 0,9581 & 14,4535 \\
\hline 55 & 0,9503 & 14,2384 \\
\hline 56 & 0,9544 & 14,3890 \\
\hline 57 & 0,9696 & 15,6833 \\
\hline 58 & 0,9586 & 14,8035 \\
\hline 59 & 0,9539 & 19,1358 \\
\hline 60 & 0,9759 & 22,9555 \\
\hline 61 & 0,9786 & 23,8746 \\
\hline 62 & 0,9822 & 23,1987 \\
\hline 63 & 0,9738 & 22,6732 \\
\hline 64 & 0,9859 & 24,4007 \\
\hline 65 & 1,0069 & 27,4999 \\
\hline 66 & 1,0278 & 27,3709 \\
\hline 67 & 1,0003 & 24,6413 \\
\hline 68 & 0,9978 & 27,4008 \\
\hline 70 & 0,9836 & 22,2253 \\
\hline 71 & 0,9861 & 21,8214 \\
\hline 72 & 0,9812 & 20,7024 \\
\hline
\end{tabular}


conclusão

\begin{tabular}{|c|c|c|}
\hline 73 & 0,9892 & 21,6205 \\
\hline 74 & 0,9615 & 21,2103 \\
\hline 75 & 0,9678 & 22,5218 \\
\hline 76 & 0,9508 & 21,3066 \\
\hline 77 & 1,0098 & 26,3157 \\
\hline 78 & 1,0063 & 26,0351 \\
\hline 79 & 1,0101 & 26,3652 \\
\hline 80 & 1,0354 & 28,7090 \\
\hline 81 & 1,0169 & 27,8140 \\
\hline 82 & 0,9903 & 26,8977 \\
\hline 83 & 0,9867 & 28,0804 \\
\hline 84 & 0,9827 & 30,5966 \\
\hline 85 & 0,9883 & 32,1390 \\
\hline 86 & 0,9876 & 30,8159 \\
\hline 87 & 1,0119 & 31,1085 \\
\hline 88 & 0,9901 & 35,2666 \\
\hline 89 & 1,0071 & 39,3133 \\
\hline 90 & 0,9838 & 32,9727 \\
\hline 91 & 0,9817 & 32,9535 \\
\hline 92 & 0,9954 & 33,4441 \\
\hline 93 & 0,9881 & 30,4762 \\
\hline 94 & 0,9900 & 28,3499 \\
\hline 95 & 0,9803 & 27,3774 \\
\hline 96 & 0,9920 & 27,2106 \\
\hline 97 & 1,0088 & 27,6060 \\
\hline 98 & 1,0194 & 27,1428 \\
\hline 99 & 1,0128 & 26,7064 \\
\hline 100 & 1,0139 & 27,7785 \\
\hline 101 & 0,9920 & 29,3135 \\
\hline 102 & 0,9929 & 31,9678 \\
\hline 103 & 0,9951 & 24,2040 \\
\hline 104 & 0,9703 & 21,3634 \\
\hline 105 & 0,9663 & 20,2244 \\
\hline 106 & 0,9606 & 20,0009 \\
\hline 107 & 0,9473 & 17,2562 \\
\hline 108 & 0,9675 & 19,0327 \\
\hline 109 & 0,9683 & 18,5834 \\
\hline 110 & 0,9742 & 17,7444 \\
\hline 111 & 0,9804 & 19,4003 \\
\hline 112 & 0,9683 & 14,8121 \\
\hline 113 & 0,9848 & 13,8219 \\
\hline 114 & 0,9624 & 14,3313 \\
\hline 115 & 0,9621 & 14,3251 \\
\hline 116 & 0,9860 & 27,0085 \\
\hline 117 & 0,9716 & 10,7620 \\
\hline 118 & 0,9533 & 21,4960 \\
\hline Perdas & \multicolumn{2}{|c|}{$131.5627 \mathrm{MW}$} \\
\hline
\end{tabular}


TABELA B.2 - Solução do sistema IEEE 118 barras estimada pela SFPO após a perturbação

\begin{tabular}{|c|c|c|}
\hline Barra k & $\mathrm{V}_{\mathrm{k}}$ (p.u.) & $\theta_{\mathrm{k}}$ (graus) \\
\hline 69 & 1,0184 & 30,0000 \\
\hline 1 & 0,9594 & 10,7611 \\
\hline 2 & 0,9721 & 11,3545 \\
\hline 3 & 0,9694 & 11,6914 \\
\hline 4 & 1,0004 & 15,4177 \\
\hline 5 & 0,9990 & 15,9383 \\
\hline 6 & 0,9916 & 13,1357 \\
\hline 7 & 0,9897 & 12,7029 \\
\hline 8 & 1,0050 & 21,1245 \\
\hline 9 & 1,0388 & 28,4539 \\
\hline 10 & 1,0527 & 36,0151 \\
\hline 11 & 0,9841 & 12,8791 \\
\hline 12 & 0,9884 & 12,3655 \\
\hline 13 & 0,9678 & 11,4707 \\
\hline 14 & 0,9828 & 11,6103 \\
\hline 15 & 0,9716 & 11,2116 \\
\hline 16 & 0,9805 & 12,0590 \\
\hline 17 & 0,9878 & 13,8783 \\
\hline 18 & 0,9711 & 11,5607 \\
\hline 19 & 0,9661 & 11,0092 \\
\hline 20 & 0,9603 & 11,8841 \\
\hline 21 & 0,9604 & 13,4653 \\
\hline 22 & 0,9710 & 16,0115 \\
\hline 23 & 1,0001 & 20,9233 \\
\hline 24 & 0,9961 & 20,7395 \\
\hline 25 & 1,0423 & 27,9835 \\
\hline 26 & 1,0190 & 29,8266 \\
\hline 27 & 0,9696 & 15,2809 \\
\hline 28 & 0,9620 & 13,5866 \\
\hline 29 & 0,9622 & 12,6255 \\
\hline 30 & 1,0054 & 19,0337 \\
\hline 31 & 0,9655 & 12,7560 \\
\hline 32 & 0,9671 & 14,7097 \\
\hline 33 & 0,9708 & 10,6169 \\
\hline 34 & 0,9863 & 11,2302 \\
\hline 35 & 0,9830 & 10,7761 \\
\hline 36 & 0,9835 & 10,7621 \\
\hline 37 & 0,9885 & 11,7587 \\
\hline 38 & 1,0014 & 17,1021 \\
\hline 39 & 0,9717 & 8,2340 \\
\hline 40 & 0,9740 & 7,0820 \\
\hline 41 & 0,9697 & 6,6353 \\
\hline 42 & 0,9844 & 8,2181 \\
\hline 43 & 0,9787 & 11,0970 \\
\hline 44 & 0,9848 & 13,4553 \\
\hline 45 & 0,9862 & 15,2409 \\
\hline 46 & 1,0065 & 17,9883 \\
\hline 47 & 1,0129 & 20,3078 \\
\hline 48 & 1,0179 & 19,4847 \\
\hline 49 & 1,0212 & 20,5092 \\
\hline 50 & 0,9981 & 18,3931 \\
\hline 51 & 0,9655 & 15,6719 \\
\hline
\end{tabular}


continuação

\begin{tabular}{|c|c|c|}
\hline 52 & 0,9562 & 14,6865 \\
\hline 53 & 0,9474 & 13,6403 \\
\hline 54 & 0,9579 & 14,4976 \\
\hline 55 & 0,9501 & 14,2822 \\
\hline 56 & 0,9542 & 14,4329 \\
\hline 57 & 0,9694 & 15,7290 \\
\hline 58 & 0,9583 & 14,8486 \\
\hline 59 & 0,9532 & 19,1885 \\
\hline 60 & 0,9750 & 23,0129 \\
\hline 61 & 0,9778 & 23,9336 \\
\hline 62 & 0,9815 & 23,2559 \\
\hline 63 & 0,9730 & 22,7302 \\
\hline 64 & 0,9852 & 24,4597 \\
\hline 65 & 1,0063 & 27,5610 \\
\hline 66 & 1,0272 & 27,4308 \\
\hline 67 & 0,9996 & 24,6992 \\
\hline 68 & 0,9974 & 27,4600 \\
\hline 70 & 0,9849 & 22,2748 \\
\hline 71 & 0,9873 & 21,8720 \\
\hline 72 & 0,9823 & 20,7554 \\
\hline 73 & 0,9904 & 21,6718 \\
\hline 74 & 0,9623 & 21,2829 \\
\hline 75 & 0,9685 & 22,5951 \\
\hline 76 & 0,9504 & 21,4220 \\
\hline 77 & 1,0104 & 26,4419 \\
\hline 78 & 1,0068 & 26,1636 \\
\hline 79 & 1,0106 & 26,4972 \\
\hline 80 & 1,0356 & 28,8502 \\
\hline 81 & 1,0167 & 27,9024 \\
\hline 82 & 0,9911 & 27,1024 \\
\hline 83 & 0,9875 & 28,3065 \\
\hline 84 & 0,9838 & 30,8546 \\
\hline 85 & 0,9896 & 32,4113 \\
\hline 86 & 0,9889 & 31,0911 \\
\hline 87 & 1,0131 & 31,3826 \\
\hline 88 & 0,9913 & 35,5660 \\
\hline 89 & 1,0084 & 39,6265 \\
\hline 90 & 0,9851 & 33,3081 \\
\hline 91 & 0,9830 & 33,2942 \\
\hline 92 & 0,9966 & 33,7957 \\
\hline 93 & 0,9891 & 30,7877 \\
\hline 94 & 0,9909 & 28,6249 \\
\hline 95 & 0,9811 & 27,6293 \\
\hline 96 & 0,9927 & 27,4314 \\
\hline 97 & 1,0092 & 27,7874 \\
\hline 98 & 1,0199 & 27,3373 \\
\hline 99 & 1,0135 & 26,9501 \\
\hline 100 & 1,0147 & 28,0606 \\
\hline 101 & 0,9940 & 29,6905 \\
\hline 102 & 0,9960 & 32,4215 \\
\hline 103 & 0,9962 & 24,4906 \\
\hline 104 & 0,9714 & 21,6572 \\
\hline 105 & 0,9675 & 20,5200 \\
\hline 106 & 0,9617 & 20,2975 \\
\hline 107 & 0,9484 & 17,5599 \\
\hline
\end{tabular}


conclusão

\begin{tabular}{|c|c|c||}
\hline 108 & 0,9690 & 19,3266 \\
\hline 109 & 0,9699 & 18,8768 \\
\hline 110 & 0,9761 & 18,0358 \\
\hline 111 & 0,9825 & 19,6822 \\
\hline 112 & 0,9706 & 15,1082 \\
\hline 113 & 0,9854 & 13,8770 \\
\hline 114 & 0,9632 & 14,3901 \\
\hline 115 & 0,9629 & 14,3840 \\
\hline 116 & 0,9854 & 27,0682 \\
\hline 117 & 0,9722 & 10,8196 \\
\hline 118 & $0,9536 \quad 131,5481 \mathrm{MW}$ \\
\hline Perdas
\end{tabular}

\section{SISTEMA IEEE 162 BARRAS}

TABELA B.3 - Solução ótima do sistema IEEE 162 barras obtida pelo FPO

\begin{tabular}{|c|c|c|}
\hline Barra k & $\mathrm{V}_{\mathrm{k}}$ (p.u.) & $\theta_{\mathrm{k}}$ (graus) \\
\hline 118 & $1,1,0422$ & $-28,0000$ \\
\hline 1 & 1,0548 & $-25,2227$ \\
\hline 2 & 1,0436 & $-29,6876$ \\
\hline 3 & 1,0269 & $-32,1602$ \\
\hline 4 & 1,0420 & $-33,3003$ \\
\hline 5 & 1,0521 & $-24,3798$ \\
\hline 6 & 1,0739 & $-19,8734$ \\
\hline 7 & 1,0394 & $-30,0269$ \\
\hline 8 & 1,0315 & $-33,4505$ \\
\hline 9 & 1,0435 & $-27,4721$ \\
\hline 10 & 1,0018 & $-35,3344$ \\
\hline 11 & 1,0084 & $-31,5518$ \\
\hline 12 & 1,0361 & $-33,2226$ \\
\hline 13 & 1,0325 & $-30,3952$ \\
\hline 14 & 1,0286 & $-30,7115$ \\
\hline 15 & 1,0343 & $-24,4800$ \\
\hline 16 & 1,0043 & $-28,9636$ \\
\hline 17 & 0,9940 & $-28,0818$ \\
\hline 18 & 1,0043 & $-32,9792$ \\
\hline 19 & 1,0011 & $-37,4291$ \\
\hline 20 & 0,9800 & $-32,4565$ \\
\hline 21 & 0,9965 & $-29,6452$ \\
\hline 22 & 1,0096 & $-36,8241$ \\
\hline 23 & 0,9865 & $-34,3788$ \\
\hline 24 & 1,0072 & $-32,8445$ \\
\hline 25 & 1,0202 & $-29,0427$ \\
\hline 26 & 1,0414 & $-21,2493$ \\
\hline 27 & 1,0225 & $-30,1089$ \\
\hline 28 & 0,9866 & $-35,6414$ \\
\hline 29 & 0,9863 & $-37,4387$ \\
\hline
\end{tabular}


continuação

\begin{tabular}{|c|c|c|}
\hline 30 & 0,9771 & $-39,2989$ \\
\hline 31 & 0,9992 & $-36,6227$ \\
\hline 32 & 0,9829 & $-40,6047$ \\
\hline 33 & 0,9799 & $-42,5831$ \\
\hline 34 & 0,9855 & $-42,5277$ \\
\hline 35 & 0,9697 & $-44,0188$ \\
\hline 36 & 0,9806 & $-42,7421$ \\
\hline 37 & 1,0350 & $-30,3365$ \\
\hline 38 & 1,0047 & $-37,4693$ \\
\hline 39 & 1,0379 & $-32,9040$ \\
\hline 40 & 0,9802 & $-41,5317$ \\
\hline 41 & 0,9911 & $-39,6834$ \\
\hline 42 & 1,0445 & $-32,7367$ \\
\hline 43 & 1,0398 & $-35,2550$ \\
\hline 44 & 1,0359 & $-35,7804$ \\
\hline 45 & 0,9932 & $-35,7708$ \\
\hline 46 & 1,0104 & $-38,5171$ \\
\hline 47 & 1,0037 & $-41,1702$ \\
\hline 48 & 1,0158 & $-40,0518$ \\
\hline 49 & 1,0015 & $-41,4840$ \\
\hline 50 & 1,0181 & $-38,9596$ \\
\hline 51 & 1,0107 & $-37,7701$ \\
\hline 52 & 1,0252 & $-38,7258$ \\
\hline 53 & 0,9975 & $-30,4322$ \\
\hline 54 & 0,9859 & $-37,4535$ \\
\hline 55 & 0,9973 & $-30,1371$ \\
\hline 56 & 0,9854 & $-40,0616$ \\
\hline 57 & 0,9957 & $-37,1281$ \\
\hline 58 & 1,0239 & $-28,5141$ \\
\hline 59 & 0,9979 & $-32,7100$ \\
\hline 60 & 0,9909 & $-33,6125$ \\
\hline 61 & 1,0035 & $-31,1628$ \\
\hline 62 & 1,0424 & $-18,6728$ \\
\hline 63 & 1,0083 & $-29,5574$ \\
\hline 64 & 1,0291 & $-28,7066$ \\
\hline 65 & 1,0154 & $-25,2871$ \\
\hline 66 & 1,0105 & $-30,8518$ \\
\hline 67 & 0,9905 & $-41,2979$ \\
\hline 68 & 1,0069 & $-39,7274$ \\
\hline 69 & 1,0148 & $-38,3925$ \\
\hline 70 & 1,0170 & $-23,2459$ \\
\hline 71 & 0,9897 & $-31,5008$ \\
\hline 72 & 1,0264 & $-30,4675$ \\
\hline 73 & 1,0037 & $-18,2970$ \\
\hline 74 & 1,0338 & $-33,1049$ \\
\hline 75 & 1,0440 & $-25,1936$ \\
\hline 76 & 1,0509 & $-16,7152$ \\
\hline 77 & 1,0036 & $-39,9703$ \\
\hline 78 & 1,0191 & $-37,7071$ \\
\hline 79 & 1,0287 & $-35,6253$ \\
\hline 80 & 1,0072 & $-37,8981$ \\
\hline 81 & 0,9805 & $-46,1047$ \\
\hline 82 & 0,9800 & $-41,5165$ \\
\hline 83 & 0,9856 & $-40,6100$ \\
\hline 84 & 1,0026 & $-37,3263$ \\
\hline
\end{tabular}


continuação

\begin{tabular}{|c|c|c|}
\hline v85 & 0,9763 & $-40,6987$ \\
\hline 86 & 0,9801 & $-44,4779$ \\
\hline 87 & 0,9920 & $-43,0233$ \\
\hline 88 & 1,0001 & $-44,1587$ \\
\hline 89 & 0,9781 & $-45,5982$ \\
\hline 90 & 0,9505 & $-48,1496$ \\
\hline 91 & 1,0291 & $-36,1932$ \\
\hline 92 & 1,0245 & $-37,0956$ \\
\hline 93 & 1,0339 & $-32,4204$ \\
\hline 94 & 1,0445 & $-36,4320$ \\
\hline 95 & 1,0292 & $-36,2688$ \\
\hline 96 & 0,9995 & $-44,6374$ \\
\hline 97 & 1,0291 & $-36,8407$ \\
\hline 98 & 1,0292 & $-34,9887$ \\
\hline 99 & 1,0543 & $-31,5251$ \\
\hline 100 & 0,9805 & $-45,3954$ \\
\hline 101 & 1,0256 & $-39,9067$ \\
\hline 102 & 1,0291 & $-36,6160$ \\
\hline 103 & 1,0477 & $-34,3681$ \\
\hline 104 & 0,9818 & $-44,1953$ \\
\hline 105 & 1,0017 & $-37,9325$ \\
\hline 106 & 1,0079 & $-42,3653$ \\
\hline 107 & 1,0097 & $-43,1799$ \\
\hline 109 & 1,0523 & $-32,7999$ \\
\hline 110 & 1,0339 & $-29,1934$ \\
\hline 111 & 1,0154 & $-33,4996$ \\
\hline 112 & 1,0425 & $-26,7494$ \\
\hline 113 & 1,0333 & $-30,6266$ \\
\hline 114 & 1,0101 & $-23,6650$ \\
\hline 115 & 1,0344 & $-35,5366$ \\
\hline 116 & 1,0307 & $-36,6831$ \\
\hline 117 & 1,0264 & $-37,3699$ \\
\hline 118 & 1,0514 & $-33,9384$ \\
\hline 119 & 1,0377 & $-34,7654$ \\
\hline 120 & 1,0400 & $-27,0971$ \\
\hline 121 & 1,0644 & $-20,6551$ \\
\hline 122 & 1,0121 & $-44,9908$ \\
\hline 123 & 1,0287 & $-45,2732$ \\
\hline 124 & 1,0444 & $-30,9259$ \\
\hline 125 & 1,0614 & $-29,4005$ \\
\hline 126 & 1,0416 & $-26,5481$ \\
\hline 127 & 1,0189 & $-29,2703$ \\
\hline 128 & 1,0384 & $-26,9970$ \\
\hline 129 & 1,0398 & $\begin{array}{l}-27,2241 \\
\end{array}$ \\
\hline 130 & 1,0654 & $-19,5353$ \\
\hline 131 & 1,0591 & $-20,6223$ \\
\hline 132 & 1,0296 & $-30,0511$ \\
\hline 133 & 1,0252 & $-31,7882$ \\
\hline 134 & 1,0318 & $-30,5282$ \\
\hline 135 & 1,0236 & $-31,8569$ \\
\hline 136 & 1,0193 & $-32,1517$ \\
\hline 137 & 1,0217 & $-32,1071$ \\
\hline 138 & 1,0305 & $-30,9884$ \\
\hline 139 & 1,0230 & $-31,7310$ \\
\hline 140 & 1,0221 & $-32,0439$ \\
\hline
\end{tabular}


conclusão

\begin{tabular}{||c|c|c||}
\hline 141 & 1,0134 & $-33,5071$ \\
\hline 142 & 0,9917 & $-39,9920$ \\
\hline 143 & 0,9818 & $-38,9417$ \\
\hline 144 & 1,0058 & $-35,6917$ \\
\hline 145 & 1,0103 & $-34,0323$ \\
\hline 146 & 0,9850 & $-39,4332$ \\
\hline 147 & 1,0205 & $-38,3587$ \\
\hline 148 & 1,0200 & $-39,3655$ \\
\hline 149 & 1,0176 & $-23,4310$ \\
\hline 150 & 1,0155 & $-24,0882$ \\
\hline 151 & 1,0052 & $-26,4119$ \\
\hline 153 & 1,0223 & $-26,4977$ \\
\hline 154 & 1,0096 & $-25,4774$ \\
\hline 155 & 0,9714 & $-31,8210$ \\
\hline 156 & 0,9806 & $-29,9941$ \\
\hline 157 & 0,9754 & $-31,5145$ \\
\hline 158 & 0,9802 & $-32,3113$ \\
\hline 159 & 0,9665 & $-33,2329$ \\
\hline 160 & 0,9663 & $-33,0529$ \\
\hline 161 & 0,9681 & $-32,4791$ \\
\hline 162 & 1,0012 & $-27,8265$ \\
\hline Perdas & 0,9990 & $-28,7118$ \\
\hline
\end{tabular}

TABELA B.4 - Solução do sistema IEEE 162 barras estimada pela SFPO após a perturbação

\begin{tabular}{|c|c|c|}
\hline Barra k & $\mathrm{V}_{\mathrm{k}}$ (p.u.) & $\theta_{\mathrm{k}}$ (graus) \\
\hline 118 & 1,0433 & $-28,0000$ \\
\hline 1 & 1,0546 & $-26,8364$ \\
\hline 2 & 1,0426 & $-31,3370$ \\
\hline 3 & 1,0263 & $-33,7190$ \\
\hline 4 & 1,0413 & $-34,8429$ \\
\hline 5 & 1,0519 & $-26,0150$ \\
\hline 6 & 1,0743 & $-21,4879$ \\
\hline 7 & 1,0384 & $-31,6910$ \\
\hline 8 & 1,0304 & $-35,1528$ \\
\hline 9 & 1,0429 & $-29,1243$ \\
\hline 10 & 0,9990 & $-36,9495$ \\
\hline 11 & 1,0058 & $-33,1041$ \\
\hline 12 & 1,0349 & $-34,9162$ \\
\hline 13 & 1,0304 & $-32,0004$ \\
\hline 14 & 1,0281 & $-32,4054$ \\
\hline 15 & 1,0312 & $-25,8967$ \\
\hline 16 & 1,0010 & $-30,0871$ \\
\hline 17 & 0,9906 & $-29,1157$ \\
\hline 18 & 1,0010 & $-34,1378$ \\
\hline 19 & 0,9986 & $-38,4807$ \\
\hline 20 & 0,9783 & $-34,0612$ \\
\hline 21 & 0,9935 & $-30,6050$ \\
\hline 22 & 1,0071 & $-37,8080$ \\
\hline 23 & 0,9836 & $-35,8929$ \\
\hline 24 & 1,0049 & $-34,3290$ \\
\hline
\end{tabular}


continuação

\begin{tabular}{|c|c|c|}
\hline 25 & 1,0183 & $-30,4886$ \\
\hline 26 & 1,0415 & $-22,8216$ \\
\hline 27 & 1,0198 & $-31,4369$ \\
\hline 28 & 0,9837 & $-37,1099$ \\
\hline 29 & 0,9831 & $-38,8614$ \\
\hline 30 & 0,9732 & $-40,6225$ \\
\hline 31 & 0,9961 & $-38,0407$ \\
\hline 32 & 0,9790 & $-41,9221$ \\
\hline 33 & 0,9763 & $-43,9353$ \\
\hline 34 & 0,9824 & $-43,8741$ \\
\hline 35 & 0,9660 & $-45,2878$ \\
\hline 36 & 0,9772 & $-44,1556$ \\
\hline 37 & 1,0325 & $-31,4253$ \\
\hline 38 & 1,0022 & $-38,4464$ \\
\hline 39 & 1,0362 & $-33,8427$ \\
\hline 40 & 0,9769 & $-42,6249$ \\
\hline 41 & 0,9883 & $-40,6235$ \\
\hline 42 & 1,0436 & $-33,5882$ \\
\hline 43 & 1,0386 & $-36,3304$ \\
\hline 44 & 1,0347 & $-36,8205$ \\
\hline 45 & 0,9907 & $-37,3009$ \\
\hline 46 & 1,0075 & $-40,1372$ \\
\hline 47 & 1,0015 & $-42,7939$ \\
\hline 48 & 1,0144 & $-41,6712$ \\
\hline 49 & 0,9992 & $-43,1079$ \\
\hline 50 & 1,0170 & $-40,5691$ \\
\hline 51 & 1,0096 & $-39,4113$ \\
\hline 52 & 1,0246 & $-40,2872$ \\
\hline 53 & 0,9958 & $-31,9983$ \\
\hline 54 & 0,9834 & $-39,0111$ \\
\hline 55 & 0,9957 & $-31,7057$ \\
\hline 56 & 0,9827 & $-41,5947$ \\
\hline 57 & 0,9937 & $-38,7042$ \\
\hline 58 & 1,0206 & $-29,9967$ \\
\hline 59 & 0,9946 & $-34,2671$ \\
\hline 60 & 0,9871 & $-35,1592$ \\
\hline 61 & 0,9997 & $-32,6742$ \\
\hline 62 & 1,0393 & $-19,9675$ \\
\hline 63 & 1,0046 & $-31,0395$ \\
\hline 64 & 1,0259 & $-30,1699$ \\
\hline 65 & 1,0119 & $-26,6460$ \\
\hline 66 & 1,0077 & $-32,3836$ \\
\hline 67 & 0,9876 & $-42,7885$ \\
\hline 68 & 1,0047 & $-41,2358$ \\
\hline 69 & 1,0130 & $-39,8972$ \\
\hline 70 & 1,0172 & $-24,8342$ \\
\hline 71 & 0,9888 & $-33,1034$ \\
\hline 72 & 1,0259 & $-32,1595$ \\
\hline 73 & 1,0054 & $-19,8947$ \\
\hline 74 & 1,0329 & $-34,6235$ \\
\hline 75 & 1,0437 & $-26,8367$ \\
\hline 76 & 1,0515 & $-18,2907$ \\
\hline 77 & 1,0013 & $-41,4333$ \\
\hline 78 & 1,0175 & $-39,2378$ \\
\hline 79 & 1,0275 & $-37,1462$ \\
\hline
\end{tabular}


continuação

\begin{tabular}{|c|c|c|}
\hline 80 & 1,0053 & $-39,4556$ \\
\hline 81 & 0,9766 & $-47,2130$ \\
\hline 82 & 0,9768 & $-42,5752$ \\
\hline 83 & 0,9826 & $-41,6097$ \\
\hline 84 & 1,0004 & $-38,0635$ \\
\hline 85 & 0,9742 & $-42,3172$ \\
\hline 86 & 0,9775 & $-46,0823$ \\
\hline 87 & 0,9896 & $-44,6458$ \\
\hline 88 & 0,9982 & $-45,6495$ \\
\hline 89 & 0,9751 & $-47,1958$ \\
\hline 90 & 0,9469 & $-49,7067$ \\
\hline 91 & 1,0280 & $-36,9880$ \\
\hline 92 & 1,0234 & $-38,0069$ \\
\hline 93 & 1,0336 & $-32,8682$ \\
\hline 94 & 1,0434 & $-37,4965$ \\
\hline 95 & 1,0281 & $-37,0328$ \\
\hline 96 & 0,9966 & $-45,9394$ \\
\hline 97 & 1,0275 & $-37,8265$ \\
\hline 98 & 1,0286 & $-35,5676$ \\
\hline 99 & 1,0532 & $-32,2794$ \\
\hline 100 & 0,9772 & $-46,7427$ \\
\hline 101 & 1,0232 & $-41,1837$ \\
\hline 102 & 1,0280 & $-37,6013$ \\
\hline 103 & 1,0466 & $-35,4641$ \\
\hline 104 & 0,9786 & $-45,5558$ \\
\hline 105 & 0,9988 & $-38,8163$ \\
\hline 106 & 1,0064 & $-43,8448$ \\
\hline 107 & 1,0080 & $-44,5829$ \\
\hline 109 & 1,0514 & $-33,8581$ \\
\hline 110 & 1,0335 & $-30,8654$ \\
\hline 111 & 1,0147 & $-35,1609$ \\
\hline 112 & 1,0423 & $-28,4036$ \\
\hline 113 & 1,0329 & $-32,3185$ \\
\hline 114 & 1,0096 & $-25,3321$ \\
\hline 115 & 1,0337 & $-37,0923$ \\
\hline 116 & 1,0299 & $-38,2133$ \\
\hline 117 & 1,0256 & $-38,9281$ \\
\hline 118 & 1,0527 & $-35,5030$ \\
\hline 119 & 1,0369 & $-36,2642$ \\
\hline 120 & 1,0396 & $-28,7557$ \\
\hline 121 & 1,0650 & $-22,3118$ \\
\hline 122 & 1,0105 & $-46,4158$ \\
\hline 123 & 1,0273 & $-46,6997$ \\
\hline 124 & 1,0434 & $-31,9899$ \\
\hline 125 & 1,0611 & $-30,6180$ \\
\hline 126 & 1,0390 & $-27,7492$ \\
\hline 127 & 1,0160 & $-30,3435$ \\
\hline 128 & 1,0381 & $-28,6555$ \\
\hline 129 & 1,0394 & $-28,8842$ \\
\hline 130 & 1,0655 & $-21,1772$ \\
\hline 131 & 1,0589 & $-22,2560$ \\
\hline 132 & 1,0291 & $-31,7389$ \\
\hline 133 & 1,0246 & $-33,4899$ \\
\hline 134 & 1,0313 & $-32,2178$ \\
\hline 135 & 1,0230 & $-33,5587$ \\
\hline
\end{tabular}


conclusão

\begin{tabular}{|c|c|c|}
\hline 136 & 1,0186 & $-33,8561$ \\
\hline 137 & 1,0211 & $-33,8114$ \\
\hline 138 & 1,0300 & $-32,6782$ \\
\hline 139 & 1,0224 & $-33,4298$ \\
\hline 140 & 1,0215 & $-33,7471$ \\
\hline 141 & 1,0126 & $-35,1751$ \\
\hline 142 & 0,9905 & $-41,6832$ \\
\hline 143 & 0,9805 & $-40,6525$ \\
\hline 144 & 1,0049 & $-37,3823$ \\
\hline 146 & 1,0095 & $-35,7295$ \\
\hline 147 & 0,9838 & $-41,1479$ \\
\hline 148 & 1,0196 & $-39,9208$ \\
\hline 149 & 1,0190 & $-40,9271$ \\
\hline 150 & 1,0177 & $-25,0186$ \\
\hline 151 & 1,0155 & $-25,6769$ \\
\hline 152 & 1,0047 & $-27,9959$ \\
\hline 153 & 1,0221 & $-28,1281$ \\
\hline 154 & 1,0094 & $-27,0787$ \\
\hline 155 & 0,9700 & $-33,4545$ \\
\hline 156 & 0,9795 & $-31,6164$ \\
\hline 157 & 0,9740 & $-33,1425$ \\
\hline 158 & 0,9786 & $-33,9185$ \\
\hline 159 & 0,9648 & $-34,8654$ \\
\hline 160 & 0,9647 & $-34,6908$ \\
\hline 161 & 0,9666 & $-34,1169$ \\
\hline 162 & 1,0002 & $-29,4082$ \\
\hline Perdas & 0,9978 & $-30,2894$ \\
\hline & & $161,0412 \mathrm{MW}$ \\
\hline
\end{tabular}

\section{SISTEMA IEEE 300 BARRAS}

TABELA B.5 - Solução ótima do sistema IEEE 300 barras obtida pelo FPO para $(\varepsilon=0,0)$ e utilizada na SFPO

\begin{tabular}{|c|c|c|}
\hline Barra $\mathrm{k}$ & $\mathrm{V}_{\mathrm{k}}$ (p.u.) & $\theta_{\mathrm{k}}$ (graus) \\
\hline \hline 7049 & 1,0804 & 0,0000 \\
\hline 1 & 1,0107 & 7,0130 \\
\hline 2 & 1,0115 & 8,9690 \\
\hline 3 & 1,0084 & 7,7257 \\
\hline 4 & 1,0163 & 5,7207 \\
\hline 5 & 0,9994 & 5,7193 \\
\hline 6 & 1,0067 & 8,1761 \\
\hline 7 & 1,0089 & 7,2849 \\
\hline 8 & 0,9873 & 3,4814 \\
\hline 9 & 0,9895 & 3,7894 \\
\hline 10 & 1,0104 & 2,3530 \\
\hline 11 & 0,9974 & 3,3516 \\
\hline 13 & 1,0083 & 6,3284 \\
\hline & 0,9860 & 0,3545 \\
\hline
\end{tabular}


continuação

\begin{tabular}{|c|c|c|}
\hline 14 & 0,9817 & $-4,1482$ \\
\hline 15 & 1,0292 & $-8,0991$ \\
\hline 16 & 1,0329 & $-1,8645$ \\
\hline 17 & 1,0340 & $-13,8262$ \\
\hline 19 & 0,9990 & 2,1848 \\
\hline 20 & 0,9860 & $-1,5367$ \\
\hline 21 & 0,9953 & 2,7296 \\
\hline 22 & 0,9835 & $-1,0805$ \\
\hline 23 & 1,0458 & 4,7944 \\
\hline 24 & 1,0154 & 7,0364 \\
\hline 25 & 1,0180 & 2,2922 \\
\hline 26 & 0,9914 & $-0,8820$ \\
\hline 27 & 0,9643 & $-4,0733$ \\
\hline 33 & 1,0361 & $-11,2573$ \\
\hline 34 & 1,0477 & $\begin{array}{l}-7,1572 \\
\end{array}$ \\
\hline 35 & 1,0015 & $-24,8741$ \\
\hline 36 & 1,0113 & $-21,7111$ \\
\hline 37 & 1,0363 & $-10,5952$ \\
\hline 38 & 1,0320 & $-11,7959$ \\
\hline 39 & 1,0599 & $-5,0441$ \\
\hline 40 & 1,0346 & $-12,0306$ \\
\hline 41 & 1,0411 & $\begin{array}{l}-9,7049 \\
\end{array}$ \\
\hline 42 & 1,0506 & $-6,6554$ \\
\hline 43 & 1,0050 & $-15,8687$ \\
\hline 44 & 1,0087 & $-16,4877$ \\
\hline 45 & 1,0184 & $-13,7873$ \\
\hline 46 & 1,0372 & $-10,8432$ \\
\hline 47 & 0,9819 & $-22,1845$ \\
\hline 48 & 1,0152 & $-15,3711$ \\
\hline 49 & 1,0733 & $-2,7846$ \\
\hline 51 & 1,0384 & $-7,5109$ \\
\hline 52 & 0,9965 & $-10,8732$ \\
\hline 53 & 0,9978 & $-16,6490$ \\
\hline 54 & 1,0023 & $-15,2320$ \\
\hline 55 & 1,0027 & $-10,9986$ \\
\hline 57 & 1,0123 & $-6,4907$ \\
\hline 58 & 0,9743 & $-4,6088$ \\
\hline 59 & 0,9626 & $-3,9261$ \\
\hline 60 & 1,0127 & $-8,4463$ \\
\hline 61 & 0,9754 & $-2,0627$ \\
\hline 62 & 0,9915 & 0,4340 \\
\hline 63 & 0,9524 & $-16,5733$ \\
\hline 64 & 0,9485 & $-11,9719$ \\
\hline 69 & 0,9632 & $-25,4734$ \\
\hline 70 & 0,9418 & $-33,7950$ \\
\hline 71 & 0,9708 & $-28,4330$ \\
\hline 72 & 0,9866 & $-26,4604$ \\
\hline 73 & 0,9890 & $-24,7546$ \\
\hline 74 & 1,0037 & $-21,0464$ \\
\hline 76 & 0,9957 & $-25,8292$ \\
\hline 77 & 1,0035 & $-24,0671$ \\
\hline 78 & 1,0079 & $-23,2125$ \\
\hline 79 & 0,9958 & $-24,1170$ \\
\hline 80 & 0,9961 & $-23,8863$ \\
\hline 81 & 1,0389 & $-17,8333$ \\
\hline
\end{tabular}


continuação

\begin{tabular}{|c|c|c|}
\hline 84 & 1,0382 & $-16,5346$ \\
\hline 85 & 0,9868 & $-16,5792$ \\
\hline 86 & 0,9915 & $-13,0367$ \\
\hline 87 & 1,0015 & $-6,6754$ \\
\hline 88 & 1,0224 & $-19,9807$ \\
\hline 89 & 1,0488 & $-10,5822$ \\
\hline 90 & 1,0507 & $-10,8715$ \\
\hline 91 & 1,0795 & $\begin{array}{l}-8,9494 \\
\end{array}$ \\
\hline 92 & 1,0982 & $-6,4318$ \\
\hline 94 & 1,0173 & $-8,3889$ \\
\hline 97 & 1,0277 & $-12,3761$ \\
\hline 98 & 0,9737 & $-13,0205$ \\
\hline 99 & 0,9884 & $-19,2178$ \\
\hline 100 & 1,0007 & $-13,2614$ \\
\hline 102 & 0,9972 & $-14,0820$ \\
\hline 103 & 1,0562 & $-11,7168$ \\
\hline 104 & 0,9929 & $-16,2090$ \\
\hline 105 & 1,0505 & $-12,5716$ \\
\hline 107 & 1,0246 & $-15,3960$ \\
\hline 108 & 0,9883 & $-19,1858$ \\
\hline 109 & 0,9781 & $-25,2122$ \\
\hline 110 & 0,9786 & $-23,8590$ \\
\hline 112 & 0,9754 & $-28,2390$ \\
\hline 113 & 0,9740 & $-24,4176$ \\
\hline 114 & 0,9773 & $-28,1699$ \\
\hline 115 & 1,0068 & $-12,2690$ \\
\hline 116 & 1,0310 & $-11,2813$ \\
\hline 117 & 0,9962 & $\begin{array}{l}-3,8783 \\
\end{array}$ \\
\hline 118 & 0,9898 & $-3,3384$ \\
\hline 119 & 1,0766 & 5,2603 \\
\hline 120 & 0,9930 & $-7,6963$ \\
\hline 121 & 1,0026 & $-11,5028$ \\
\hline 122 & 1,0082 & $-12,8414$ \\
\hline 123 & 1,0209 & $-15,9548$ \\
\hline 124 & 1,0349 & $-11,9571$ \\
\hline 125 & 1,0309 & $-16,6767$ \\
\hline 126 & 1,0194 & $-11,3435$ \\
\hline 127 & 1,0191 & $-9,0927$ \\
\hline 128 & 1,0244 & $-3,5742$ \\
\hline 129 & 1,0255 & $-3,2210$ \\
\hline 130 & 1,0167 & 6,6604 \\
\hline 131 & 1,0108 & 7,0956 \\
\hline 132 & 1,0032 & 4,1636 \\
\hline 133 & 1,0308 & $-4,2599$ \\
\hline 134 & 1,0342 & $-6,6715$ \\
\hline 135 & 1,0294 & $-5,4238$ \\
\hline 136 & 1,0526 & 2,7190 \\
\hline 137 & 1,0556 & $-0,2021$ \\
\hline 138 & 1,0590 & $-5,0372$ \\
\hline 139 & 1,0299 & $-2,1542$ \\
\hline 140 & 1,0403 & $-2,0682$ \\
\hline 141 & 1,0595 & 1,4552 \\
\hline 142 & 1,0272 & $-1,3500$ \\
\hline 143 & 1,0657 & 5,3287 \\
\hline 144 & 0,9979 & 0,7977 \\
\hline
\end{tabular}


continuação

\begin{tabular}{|c|c|c|}
\hline 145 & 1,0124 & 1,1966 \\
\hline 146 & 1,0519 & 5,7114 \\
\hline 147 & 1,0540 & 9,7336 \\
\hline 148 & 1,0378 & 1,5591 \\
\hline 149 & 1,0751 & 6,6229 \\
\hline 150 & 1,0105 & 7,3523 \\
\hline 151 & 1,0018 & 5,2163 \\
\hline 152 & 1,0530 & 10,3505 \\
\hline 153 & 1,0283 & 11,5043 \\
\hline 154 & 1,0059 & $-0,2085$ \\
\hline 155 & 0,9863 & 7,8011 \\
\hline 156 & 0,9763 & 6,3090 \\
\hline 157 & 1,0104 & $-10,4607$ \\
\hline 158 & 1,0236 & $-9,9323$ \\
\hline 159 & 1,0087 & $-8,3978$ \\
\hline 160 & 1,0314 & $-11,0504$ \\
\hline 161 & 1,0153 & 9,8893 \\
\hline 162 & 0,9982 & 19,5434 \\
\hline 163 & 1,0653 & 4,1883 \\
\hline 164 & 0,9978 & 10,8508 \\
\hline 165 & 1,0004 & 27,3260 \\
\hline 166 & 0,9944 & 31,2510 \\
\hline 167 & 0,9911 & $-5,6203$ \\
\hline 168 & 1,0244 & $-3,5962$ \\
\hline 169 & 1,0119 & $-5,4092$ \\
\hline 170 & 0,9736 & 1,5041 \\
\hline 171 & 1,0419 & $-8,3969$ \\
\hline 172 & 1,0266 & $-4,7711$ \\
\hline 173 & 0,9827 & $-11,3359$ \\
\hline 174 & 1,0496 & $-1,2631$ \\
\hline 175 & 0,9810 & $-5,7768$ \\
\hline 176 & 1,0508 & 6,0494 \\
\hline 177 & 1,0085 & 1,9271 \\
\hline 178 & 0,9388 & $-5,2701$ \\
\hline 179 & 0,9671 & $-8,1022$ \\
\hline 180 & 0,9817 & $-1,7598$ \\
\hline 181 & 1,0590 & $-0,0648$ \\
\hline 182 & 1,0305 & $-2,7515$ \\
\hline 183 & 1,0252 & 8,3854 \\
\hline 184 & 1,0456 & $-5,4706$ \\
\hline 185 & 1,0559 & $-2,9634$ \\
\hline 186 & 1,0748 & 3,3594 \\
\hline 187 & 1,0699 & 2,6307 \\
\hline 188 & 1,0610 & 0,5229 \\
\hline 189 & 0,9615 & $-24,8597$ \\
\hline 190 & 1,0611 & $-19,4279$ \\
\hline 191 & 1,0949 & 10,4592 \\
\hline 192 & 0,9867 & $-10,6855$ \\
\hline 193 & 0,9581 & $-26,3555$ \\
\hline 194 & 1,0517 & $-18,0595$ \\
\hline 195 & 1,0402 & $-19,5632$ \\
\hline 196 & 0,9650 & $-23,1149$ \\
\hline 197 & 0,9825 & $-21,9191$ \\
\hline 198 & 1,0004 & $-18,8205$ \\
\hline 199 & 0,9442 & $-24,3509$ \\
\hline
\end{tabular}


continuação

\begin{tabular}{|c|c|c|}
\hline 200 & 0,9477 & $-24,3195$ \\
\hline 201 & 0,9417 & $-28,3219$ \\
\hline 202 & 0,9734 & $-23,9102$ \\
\hline 203 & 0,9916 & $-20,7604$ \\
\hline 204 & 0,9246 & $-28,4806$ \\
\hline 205 & 0,9448 & $-27,4301$ \\
\hline 206 & 0,9670 & $-27,4359$ \\
\hline 207 & 0,9840 & $-27,2557$ \\
\hline 208 & 0,9590 & $-25,8830$ \\
\hline 209 & 0,9626 & $-24,4267$ \\
\hline 210 & 0,9694 & $-22,4426$ \\
\hline 211 & 0,9941 & $-21,8982$ \\
\hline 212 & 1,0048 & $-21,1359$ \\
\hline 213 & 0,9819 & $-9,9680$ \\
\hline 214 & 0,9846 & $-16,1203$ \\
\hline 215 & 0,9808 & $-18,8693$ \\
\hline 216 & 0,9721 & $-21,2267$ \\
\hline 217 & 1,0244 & $-20,9407$ \\
\hline 218 & 1,0219 & $-21,3736$ \\
\hline 219 & 1,0564 & $-19,9082$ \\
\hline 220 & 1,0099 & $-20,4308$ \\
\hline 221 & 0,9832 & $-21,1533$ \\
\hline 222 & 1,0480 & $-21,9232$ \\
\hline 223 & 0,9807 & $-21,3839$ \\
\hline 224 & 1,0002 & $-20,2634$ \\
\hline 225 & 0,9912 & $-10,8159$ \\
\hline 226 & 1,0185 & $-20,3399$ \\
\hline 227 & 0,9947 & $-25,9974$ \\
\hline 228 & 1,0337 & $-19,7120$ \\
\hline 229 & 1,0428 & $-18,7266$ \\
\hline 230 & 1,0538 & $-12,7655$ \\
\hline 231 & 1,0556 & $-19,9888$ \\
\hline 232 & 1,0424 & $-21,9554$ \\
\hline 233 & 0,9928 & $-24,6591$ \\
\hline 234 & 1,0214 & $-19,6380$ \\
\hline 235 & 1,0144 & $-19,7938$ \\
\hline 236 & 1,0353 & $-14,4564$ \\
\hline 237 & 1,0572 & $-19,8603$ \\
\hline 238 & 1,0154 & $-19,7168$ \\
\hline 239 & 1,0172 & $-14,8341$ \\
\hline 240 & 1,0294 & $-18,9520$ \\
\hline 241 & 1,0460 & $-15,2488$ \\
\hline 242 & 0,9909 & $-16,1547$ \\
\hline 243 & 1,0328 & $-18,2616$ \\
\hline 244 & 1,0108 & $-19,0888$ \\
\hline 245 & 0,9790 & $-19,5857$ \\
\hline 246 & 0,9772 & $-20,4511$ \\
\hline 247 & 0,9718 & $-20,2892$ \\
\hline 248 & 0,9759 & $-23,7103$ \\
\hline 249 & 0,9751 & $-24,1348$ \\
\hline 250 & 1,0195 & $-22,2764$ \\
\hline 281 & 1,0308 & $-18,8802$ \\
\hline 319 & 1,0250 & 2,5701 \\
\hline 320 & 1,0082 & $-1,3690$ \\
\hline 322 & 0,9979 & $-16,4732$ \\
\hline
\end{tabular}


continuação

\begin{tabular}{|c|c|c|}
\hline 323 & 0,9818 & $-12,5367$ \\
\hline 324 & 0,9725 & $-22,3859$ \\
\hline 526 & 0,9367 & $-33,4488$ \\
\hline 528 & 0,9624 & $-36,2493$ \\
\hline 531 & 0,9786 & $-28,0336$ \\
\hline 552 & 1,0194 & $-22,5433$ \\
\hline 562 & 0,9869 & $-26,9025$ \\
\hline 609 & 0,9775 & $-27,7990$ \\
\hline 664 & 1,0338 & $-15,8532$ \\
\hline 1190 & 1,0692 & 4,1298 \\
\hline 1200 & 0,9848 & $-6,3486$ \\
\hline 1201 & 1,0084 & $-14,1164$ \\
\hline 2040 & 0,9616 & $-23,5911$ \\
\hline 7001 & 1,0496 & 11,9376 \\
\hline 7002 & 1,0336 & 13,8831 \\
\hline 7003 & 0,9936 & 15,0103 \\
\hline 7011 & 1,0093 & 5,9096 \\
\hline 7012 & 1,0300 & 12,7450 \\
\hline 7017 & 1,0987 & $-11,0790$ \\
\hline 7023 & 1,0654 & 6,9831 \\
\hline 7024 & 1,0084 & 13,6812 \\
\hline 7039 & 1,0976 & 2,7616 \\
\hline 7044 & 1,0191 & $-12,7359$ \\
\hline 7055 & 1,0120 & $-6,0093$ \\
\hline 7057 & 1,0474 & $-1,7147$ \\
\hline 7061 & 1,0158 & 3,4513 \\
\hline 7062 & 0,9687 & 8,1165 \\
\hline 7071 & 0,9989 & $-23,6986$ \\
\hline 7130 & 1,0797 & 19,7902 \\
\hline 7139 & 1,0473 & 4,0675 \\
\hline 7166 & 1,0078 & 36,1258 \\
\hline 9001 & 1,0363 & $-10,6167$ \\
\hline 9002 & 0,9904 & $-17,8067$ \\
\hline 9003 & 0,9753 & $-19,6827$ \\
\hline 9004 & 0,9684 & $-19,8841$ \\
\hline 9005 & 1,0367 & $-10,6922$ \\
\hline 9006 & 0,9947 & $-17,2400$ \\
\hline 9007 & 0,9842 & $-18,5950$ \\
\hline 9012 & 1,0004 & $-16,3473$ \\
\hline 9021 & 0,9843 & $-17,9973$ \\
\hline 9022 & 0,9435 & $-21,1989$ \\
\hline 9023 & 0,9843 & $-17,9973$ \\
\hline 9024 & 0,9847 & $-19,6989$ \\
\hline 9025 & 0,9843 & $-17,9973$ \\
\hline 9026 & 0,9843 & $-17,9973$ \\
\hline 9031 & 0,9026 & $-25,2949$ \\
\hline 9032 & 0,9660 & $-22,6545$ \\
\hline 9033 & 0,9001 & $-25,4710$ \\
\hline 9034 & 0,9855 & $-21,4146$ \\
\hline 9035 & 0,9236 & $-23,7374$ \\
\hline 9036 & 0,9515 & $-22,6873$ \\
\hline 9037 & 0,9384 & $-22,6624$ \\
\hline 9038 & 0,9246 & $-25,0653$ \\
\hline 9041 & 0,9642 & $-21,3913$ \\
\hline 9042 & 0,9556 & $-23,2627$ \\
\hline
\end{tabular}


conclusão

\begin{tabular}{|c|c|c|}
\hline 9043 & 0,9465 & $-21,9112$ \\
\hline 9044 & 0,9707 & $-19,8231$ \\
\hline 9051 & 1,0782 & $-17,7556$ \\
\hline 9052 & 0,9326 & $-17,1560$ \\
\hline 9053 & 1,0584 & $-16,1359$ \\
\hline 9054 & 1,0893 & $-6,8333$ \\
\hline 9055 & 1,0366 & $-7,2780$ \\
\hline 9071 & 0,9792 & $-20,3592$ \\
\hline 9072 & 0,9808 & $-19,8161$ \\
\hline 9121 & 0,9800 & $-18,5255$ \\
\hline 9533 & $1,0584 \quad-16,5195$ \\
\hline Perdas & \multicolumn{2}{|c|}{$408,3139 \mathrm{MW}$} \\
\hline
\end{tabular}

TABELA B.6 - Solução do sistema IEEE 300 barras estimada pela SFPO após a perturbação

\begin{tabular}{|c|c|c|}
\hline Barra k & $\mathrm{V}_{\mathrm{k}}$ (p.u.) & $\theta_{\mathrm{k}}$ (graus) \\
\hline 7049 & 1,0869 & 0,0000 \\
\hline 1 & 1,0075 & 7,0462 \\
\hline 2 & 1,0087 & 9,0128 \\
\hline 3 & 1,0062 & 7,7592 \\
\hline 4 & 1,0143 & 5,7437 \\
\hline 5 & 0,9963 & 5,7437 \\
\hline 6 & 1,0039 & 8,2145 \\
\hline 7 & 1,0066 & 7,3171 \\
\hline 8 & 0,9847 & 3,4965 \\
\hline 9 & 0,9864 & 3,8041 \\
\hline 10 & 1,0081 & 2,3625 \\
\hline 11 & 0,9944 & 3,3657 \\
\hline 12 & 1,0058 & 6,3572 \\
\hline 13 & 0,9829 & 0,3507 \\
\hline 14 & 0,9795 & $-4,1664$ \\
\hline 15 & 1,0277 & $-8,1292$ \\
\hline 16 & 1,0315 & $-1,8789$ \\
\hline 17 & 1,0321 & $-13,8631$ \\
\hline 19 & 0,9965 & 2,1945 \\
\hline 20 & 0,9829 & $-1,5519$ \\
\hline 21 & 0,9926 & 2,7420 \\
\hline 22 & 0,9802 & $-1,0913$ \\
\hline 23 & 1,0420 & 4,8285 \\
\hline 24 & 1,0127 & 7,0768 \\
\hline 25 & 1,0142 & 2,3082 \\
\hline 26 & 0,9875 & $-0,8898$ \\
\hline 27 & 0,9606 & $-4,1050$ \\
\hline 33 & 1,0367 & $-11,2893$ \\
\hline 34 & 1,0474 & $-7,1952$ \\
\hline 35 & 0,9997 & $-24,9681$ \\
\hline 36 & 1,0094 & $-21,7929$ \\
\hline 37 & 1,0377 & $-10,6260$ \\
\hline 38 & 1,0327 & $-11,8295$ \\
\hline 39 & 1,0597 & $-5,0817$ \\
\hline 40 & 1,0354 & $-12,0617$ \\
\hline 41 & 1,0419 & $-9,7344$ \\
\hline
\end{tabular}


continuação

\begin{tabular}{|c|c|c|}
\hline 42 & 1,0502 & $-6,6928$ \\
\hline 43 & 1,0048 & $-15,9359$ \\
\hline 44 & 1,0081 & $-16,5668$ \\
\hline 45 & 1,0171 & $-13,8573$ \\
\hline 46 & 1,0362 & $-10,8922$ \\
\hline 47 & 0,9809 & $-22,2705$ \\
\hline 48 & 1,0153 & $-15,4193$ \\
\hline 49 & 1,0784 & $\begin{array}{l}-2,8171 \\
\end{array}$ \\
\hline 51 & 1,0398 & $-7,5714$ \\
\hline 52 & 0,9946 & $-11,0578$ \\
\hline 53 & 0,9974 & $-16,7300$ \\
\hline 54 & 1,0014 & $-15,3362$ \\
\hline 55 & 1,0006 & $-11,1587$ \\
\hline 57 & 1,0089 & $-6,5902$ \\
\hline 58 & 0,9712 & $-4,6721$ \\
\hline 59 & 0,9595 & $-3,9767$ \\
\hline 60 & 1,0095 & $-8,5002$ \\
\hline 61 & 0,9723 & $-2,0971$ \\
\hline 62 & 0,9856 & 0,4421 \\
\hline 63 & 0,9405 & $-16,8366$ \\
\hline 64 & 0,9379 & $-12,1305$ \\
\hline 69 & 0,9609 & $-25,5680$ \\
\hline 70 & 0,9400 & $-33,9243$ \\
\hline 71 & 0,9691 & $-28,5439$ \\
\hline 72 & 0,9848 & $-26,5606$ \\
\hline 73 & 0,9873 & $-24,8490$ \\
\hline 74 & 1,0019 & $-21,1286$ \\
\hline 76 & 0,9939 & $-25,9275$ \\
\hline 77 & 1,0016 & $-24,1575$ \\
\hline 78 & 1,0060 & $-23,2997$ \\
\hline 79 & 0,9939 & $-24,2076$ \\
\hline 80 & 0,9940 & $-23,9751$ \\
\hline 81 & 1,0369 & $-17,8983$ \\
\hline 84 & 1,0364 & $-16,5976$ \\
\hline 85 & 0,9861 & $-16,6348$ \\
\hline 86 & 0,9906 & $-13,0840$ \\
\hline 87 & 0,9998 & $-6,7026$ \\
\hline 88 & 1,0205 & $-20,0556$ \\
\hline 89 & 1,0482 & $-10,6149$ \\
\hline 90 & 1,0510 & $-10,9016$ \\
\hline 91 & 1,0786 & $-8,9797$ \\
\hline 92 & 1,0979 & $-6,4694$ \\
\hline 94 & 1,0158 & $-8,4180$ \\
\hline 97 & 1,0270 & $-12,4186$ \\
\hline 98 & 0,9733 & $-13,0727$ \\
\hline 99 & 0,9879 & $-19,2768$ \\
\hline 100 & 1,0001 & $-13,3085$ \\
\hline 102 & 0,9965 & $-14,1294$ \\
\hline 103 & 1,0558 & $-11,7606$ \\
\hline 104 & 0,9922 & $-16,2615$ \\
\hline 105 & 1,0501 & $-12,6172$ \\
\hline 107 & 1,0244 & $\begin{array}{l}-15,4447 \\
\end{array}$ \\
\hline 108 & 0,9878 & $-19,2455$ \\
\hline 109 & 0,9772 & $-25,2893$ \\
\hline 110 & 0,9779 & $-23,9284$ \\
\hline
\end{tabular}


continuação

\begin{tabular}{|c|c|c|}
\hline 112 & 0,9742 & $-28,3278$ \\
\hline 113 & 0,9730 & $-24,5035$ \\
\hline 114 & 0,9761 & $-28,2596$ \\
\hline 115 & 1,0043 & $-12,3162$ \\
\hline 116 & 1,0283 & $-11,3224$ \\
\hline 117 & 0,9935 & $-3,8838$ \\
\hline 118 & 0,9872 & $-3,3413$ \\
\hline 19 & 1,0748 & 5,2922 \\
\hline 120 & 0,9906 & $-7,7184$ \\
\hline 121 & 1,0002 & $-11,5447$ \\
\hline 122 & 1,0056 & $-12,8918$ \\
\hline 123 & 1,0181 & $-16,0200$ \\
\hline 124 & 1,0321 & $-12,0007$ \\
\hline 125 & 1,0282 & $-16,7468$ \\
\hline 126 & 1,0168 & $-11,3864$ \\
\hline 127 & 1,0167 & $-9,1250$ \\
\hline 128 & 1,0222 & $-3,5827$ \\
\hline 129 & 1,0234 & $-3,2281$ \\
\hline 130 & 1,0145 & 6,6920 \\
\hline 131 & 1,0085 & 7,1273 \\
\hline 132 & 1,0010 & 4,1847 \\
\hline 133 & 1,0289 & $-4,2708$ \\
\hline 134 & 1,0319 & $-6,6925$ \\
\hline 135 & 1,0272 & $-5,4402$ \\
\hline 136 & 1,0508 & 2,7313 \\
\hline 137 & 1,0540 & $-0,1976$ \\
\hline 138 & 1,0573 & $-5,0477$ \\
\hline 139 & 1,0291 & $-2,1559$ \\
\hline 140 & 1,0392 & $-2,0689$ \\
\hline 141 & 1,0590 & 1,4589 \\
\hline 142 & 1,0275 & $-1,3440$ \\
\hline 143 & 1,0663 & 5,3341 \\
\hline 144 & 0,9926 & 0,8041 \\
\hline 145 & 1,0130 & 1,2023 \\
\hline 146 & 1,0523 & 5,7123 \\
\hline 147 & 1,0543 & 9,7326 \\
\hline 148 & 1,0386 & 1,5686 \\
\hline 149 & 1,0758 & 6,6244 \\
\hline 150 & 1,0084 & 7,3852 \\
\hline 151 & 0,9996 & 5,2413 \\
\hline 152 & 1,0516 & 10,3822 \\
\hline 153 & 1,0280 & 11,5292 \\
\hline 154 & 1,0060 & $-0,1843$ \\
\hline 155 & 0,9867 & 7,8164 \\
\hline 156 & 0,9767 & 6,3252 \\
\hline 157 & 1,0078 & $-10,4993$ \\
\hline 158 & 1,0209 & $-9,9675$ \\
\hline 159 & 1,0061 & $-8,4261$ \\
\hline 160 & 1,0287 & $-11,0900$ \\
\hline 161 & 1,0152 & 9,9105 \\
\hline 162 & 0,9996 & 19,5353 \\
\hline 163 & 1,0645 & 4,2029 \\
\hline 164 & 0,9983 & 10,8629 \\
\hline 165 & 1,0024 & 27,2893 \\
\hline 166 & 0,9966 & 31,1978 \\
\hline
\end{tabular}


continuação

\begin{tabular}{|c|c|c|}
\hline 167 & 0,9889 & $-5,6386$ \\
\hline 168 & 1,0223 & $-3,6048$ \\
\hline 169 & 1,0097 & $-5,4255$ \\
\hline 170 & 0,9716 & 1,5135 \\
\hline 171 & 1,0400 & $-8,4231$ \\
\hline 172 & 1,0262 & $-4,7723$ \\
\hline 173 & 0,9832 & $-11,3287$ \\
\hline 174 & 1,0493 & $-1,2601$ \\
\hline 175 & 0,9816 & $-5,7676$ \\
\hline 176 & 1,0525 & 6,0130 \\
\hline 177 & 1,0103 & 1,9062 \\
\hline 178 & 0,9400 & $-5,2558$ \\
\hline 179 & 0,9681 & $-8,0839$ \\
\hline 180 & 0,9825 & $-1,7497$ \\
\hline 181 & 1,0573 & $-0,0595$ \\
\hline 182 & 1,0295 & $-2,7544$ \\
\hline 183 & 1,0250 & 8,4097 \\
\hline 184 & 1,0431 & $-5,4853$ \\
\hline 185 & 1,0534 & $-2,9660$ \\
\hline 186 & 1,0731 & 3,3761 \\
\hline 187 & 1,0682 & 2,6450 \\
\hline 188 & 1,0593 & 0,5301 \\
\hline 189 & 0,9590 & $-24,9482$ \\
\hline 190 & 1,0585 & $-19,4951$ \\
\hline 191 & 1,0946 & 10,4284 \\
\hline 192 & 0,9858 & $-10,7438$ \\
\hline 193 & 0,9556 & $-26,4507$ \\
\hline 194 & 1,0494 & $-18,1225$ \\
\hline 195 & 1,0379 & $-19,6324$ \\
\hline 196 & 0,9624 & $-23,1976$ \\
\hline 197 & 0,9799 & $-21,9962$ \\
\hline 198 & 0,9978 & $-18,8817$ \\
\hline 199 & 0,9413 & $-24,4393$ \\
\hline 200 & 0,9448 & $-24,4046$ \\
\hline 201 & 0,9393 & $-28,4311$ \\
\hline 202 & 0,9708 & $-23,9987$ \\
\hline 203 & 0,9891 & $-20,8319$ \\
\hline 204 & 0,9221 & $-28,5880$ \\
\hline 205 & 0,9424 & $-27,5311$ \\
\hline 206 & 0,9652 & $-27,5341$ \\
\hline 207 & 0,9824 & $-27,3541$ \\
\hline 208 & 0,9565 & $-25,9755$ \\
\hline 209 & 0,9600 & $-24,5135$ \\
\hline 210 & 0,9667 & $-22,5215$ \\
\hline 211 & 0,9916 & $-21,9764$ \\
\hline 212 & 1,0023 & $-21,2106$ \\
\hline 213 & 0,9792 & $-9,9787$ \\
\hline 214 & 0,9817 & $-16,1654$ \\
\hline 215 & 0,9779 & $-18,9308$ \\
\hline 216 & 0,9692 & $-21,3026$ \\
\hline 217 & 1,0217 & $-21,0161$ \\
\hline 218 & 1,0191 & $-21,4516$ \\
\hline 219 & 1,0537 & $-19,9782$ \\
\hline 220 & 1,0071 & $-20,5043$ \\
\hline 221 & 0,9815 & $-21,2329$ \\
\hline
\end{tabular}


continuação

\begin{tabular}{|c|c|c|}
\hline 222 & 1,0456 & $-22,0031$ \\
\hline 223 & 0,9790 & $-21,4641$ \\
\hline 224 & 0,9983 & $-20,3388$ \\
\hline 225 & 0,9902 & $-10,8736$ \\
\hline 226 & 1,0164 & $-20,4127$ \\
\hline 227 & 0,9930 & $-26,0940$ \\
\hline 228 & 1,0305 & $-19,7807$ \\
\hline 229 & 1,0395 & $-18,7886$ \\
\hline 230 & 1,0502 & $-12,7883$ \\
\hline 231 & 1,0530 & $-20,0593$ \\
\hline 232 & 1,0399 & $-22,0357$ \\
\hline 233 & 0,9910 & $-24,7524$ \\
\hline 234 & 1,0184 & $-19,7063$ \\
\hline 235 & 1,0115 & $-19,8625$ \\
\hline 236 & 1,0323 & $-14,4941$ \\
\hline 237 & 1,0546 & $-19,9301$ \\
\hline 238 & 1,0125 & $-19,7851$ \\
\hline 239 & 1,0143 & $-14,8744$ \\
\hline 240 & 1,0269 & $-19,0169$ \\
\hline 241 & 1,0436 & $\begin{array}{l}-15,2971 \\
\end{array}$ \\
\hline 242 & 0,9880 & $-16,1994$ \\
\hline 243 & 1,0289 & $-18,3044$ \\
\hline 244 & 1,0070 & $-19,1407$ \\
\hline 245 & 0,9756 & $-19,6482$ \\
\hline 246 & 0,9736 & $-20,5178$ \\
\hline 247 & 0,9685 & $-20,3571$ \\
\hline 248 & 0,9726 & $-23,7967$ \\
\hline 249 & 0,9718 & $-24,2242$ \\
\hline 250 & 1,0163 & $-22,3535$ \\
\hline 281 & 1,0283 & $-18,9447$ \\
\hline 319 & 1,0223 & 2,5866 \\
\hline 320 & 1,0044 & $-1,3804$ \\
\hline 322 & 0,9973 & $-16,5260$ \\
\hline 323 & 0,9809 & $-12,5831$ \\
\hline 324 & 0,9720 & $-22,4485$ \\
\hline 526 & 0,9234 & $-34,1750$ \\
\hline 528 & 0,9607 & $-36,3875$ \\
\hline 531 & 0,9768 & $-28,1398$ \\
\hline 552 & 1,0175 & $-22,6278$ \\
\hline 562 & 0,9850 & $-27,0053$ \\
\hline 609 & 0,9756 & $-27,9038$ \\
\hline 664 & 1,0313 & $-15,9059$ \\
\hline 1190 & 1,0674 & 4,1580 \\
\hline 1200 & 0,9824 & $-6,3642$ \\
\hline 1201 & 1,0065 & $-14,1621$ \\
\hline 2040 & 0,9590 & $-23,6759$ \\
\hline 7001 & 1,0458 & 12,0043 \\
\hline 7002 & 1,0304 & 13,9570 \\
\hline 7003 & 0,9921 & 15,0710 \\
\hline 7011 & 1,0061 & 5,9394 \\
\hline 7012 & 1,0275 & 12,8054 \\
\hline 7017 & 1,0967 & $-11,1058$ \\
\hline 7023 & 1,0611 & 7,0338 \\
\hline 7024 & 1,0063 & 13,7538 \\
\hline 7039 & 1,0984 & 2,7193 \\
\hline
\end{tabular}


conclusão

\begin{tabular}{|c|c|c|}
\hline 7044 & 1,0266 & $-12,8405$ \\
\hline 7055 & 1,0102 & $-6,1503$ \\
\hline 7057 & 1,0446 & $-1,7858$ \\
\hline 7061 & 1,0141 & 3,4432 \\
\hline 7062 & 0,9595 & 8,2442 \\
\hline 7071 & 0,9976 & $-23,7953$ \\
\hline 7130 & 1,0777 & 19,8757 \\
\hline 7139 & 1,0469 & 4,0733 \\
\hline 7166 & 1,0103 & 36,0498 \\
\hline 9001 & 1,0376 & $-10,6476$ \\
\hline 9002 & 0,9925 & $-17,8155$ \\
\hline 9003 & 0,9770 & $-19,6860$ \\
\hline 9004 & 0,9701 & $-19,8867$ \\
\hline 9005 & 1,0382 & $-10,7238$ \\
\hline 9006 & 0,9963 & $-17,2511$ \\
\hline 9007 & 0,9858 & $-18,6018$ \\
\hline 9012 & 1,0024 & $-16,3605$ \\
\hline 9021 & 0,9864 & $-18,0052$ \\
\hline 9022 & 0,9457 & $-21,1924$ \\
\hline 9023 & 0,9864 & $-18,0052$ \\
\hline 9024 & 0,9868 & $-19,6996$ \\
\hline 9025 & 0,9864 & $-18,0052$ \\
\hline 9026 & 0,9864 & $-18,0052$ \\
\hline 9031 & 0,9044 & $-25,2767$ \\
\hline 9032 & 0,9678 & $-22,6473$ \\
\hline 9033 & 0,9020 & $-25,4521$ \\
\hline 9034 & 0,9873 & $-21,4123$ \\
\hline 9035 & 0,9255 & $-23,7256$ \\
\hline 9036 & 0,9533 & $-22,6798$ \\
\hline 9037 & 0,9402 & $-22,6548$ \\
\hline 9038 & 0,9264 & $-25,0485$ \\
\hline 9041 & 0,9660 & $-21,3885$ \\
\hline 9042 & 0,9573 & $-23,2532$ \\
\hline 9043 & 0,9482 & $-21,9065$ \\
\hline 9044 & 0,9724 & $-19,8259$ \\
\hline 9051 & 1,0795 & $-17,7682$ \\
\hline 9052 & 0,9342 & $-17,1668$ \\
\hline 9053 & 1,0598 & $-16,1524$ \\
\hline 9054 & 1,0930 & $-6,8836$ \\
\hline 9055 & 1,0416 & $-7,3307$ \\
\hline 9071 & 0,9809 & $-20,3599$ \\
\hline 9072 & 0,9825 & $-19,8187$ \\
\hline 9121 & 0,9820 & $-18,5300$ \\
\hline 9533 & 1,0598 & $-16,5350$ \\
\hline Perdas & \multicolumn{2}{|c|}{ 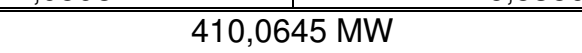 } \\
\hline
\end{tabular}

